

\section{HANDHELD LASER SPECKLE CONTRAST}

PERFUSION IMAGING 


\section{HANDHELD LASER SPECKLE CONTRAST PERFUSION IMAGING}

\section{PROEFSCHRIFT}

ter verkrijging van de graad van doctor aan de Universiteit Twente, op gezag van de rector magnificus,

Prof. dr. A. Veldkamp, volgens besluit van het College voor Promoties in het openbaar te verdedigen op donderdag 14 oktober 2021 om 12.45 uur

door

Ata Chizari

geboren op 30 december 1990

te Teheran, Iran 
Dit proefschrift is goedgekeurd door:

Promotor

prof. dr. ir. W. Steenbergen

Print and cover design: Gildeprint

ISBN: 978-90-365-5239-4

DOI: $10.3990 / 1.9789036552394$

URL: https://doi.org/10.3990/1.9789036552394

(C) 2021 Ata Chizari, The Netherlands. All rights reserved. No parts of this thesis may be reproduced, stored in a retrieval system or transmitted in any form or by any means without permission of the author. Alle rechten voorbehouden. Niets uit deze uitgave mag worden vermenigvuldigd, in enige vorm of op enige wijze, zonder voorafgaande schriftelijke toestemming van de auteur. 


\section{PROMOTIECOMMISSIE:}

Voorzitter / secretaris: Promotor:

Leden:
Universiteit Twente

Universiteit Twente

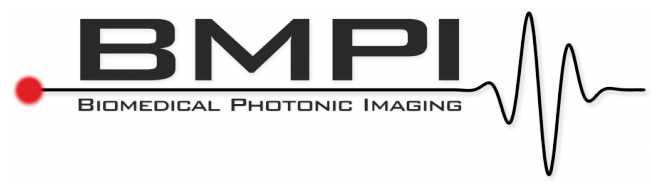

The work in this thesis was carried out at the Biomedical Photonic Imaging group of the Faculty of Science and Technology of the University of Twente. This study was supported by the Open Technology program of the Netherlands Organization for Scientific Research (NWO), Domain Applied and Engineering Sciences, under grant number 14538. The author declare that there are not any financial interests related to this thesis and no potential conflicts of interest to disclose.

Ata Chizari, Biomedical Photonic Imaging, University of Twente, P.O. Box 217, 7500 AE Enschede, The Netherlands https://www.utwente.nl/en/tnw/bmpi/

Nederlandse titel:

Draagbare laser speckle contrast perfusie beeldvorming 


\section{Contents}

1 Introduction 1

1.1 Laser speckle contrast perfusion imaging . . . . . . . . . . . 1

1.2 Thesis outline . . . . . . . . . . . . . . . 3

2 Exploration of movement artefacts in handheld laser speckle contrast $\begin{array}{ll}\text { perfusion imaging } & 7\end{array}$

2.1 Introduction . . . . . . . . . . . . . . . . . 7

2.2 Methods . . . . . . . . . . . . . . . . . . . . 9

2.3 Results . . . . . . . . . . . . . . . . . . . . . . . 14

2.4 Discussion . . . . . . . . . . . . . . . . 18

3 Influence of wavefront types on movement artefacts in handheld laser speckle contrast perfusion imaging 25

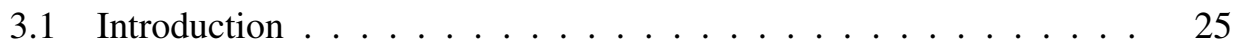

3.2 Methods and materials . . . . . . . . . . . . . . . 26

3.3 Results . . . . . . . . . . . . . . . . . 33

3.4 Discussion . . . . . . . . . . . . . . . . . 37

$\begin{array}{ll}\text { Appendices } & 45\end{array}$

$\begin{array}{lll}\text { A Supplementary information } & 47\end{array}$

4 Prediction of movement artefacts caused by translation in handheld laser speckle contrast perfusion imaging $\quad 51$

4.1 Introduction . . . . . . . . . . . . . . . 52

4.2 Model of the optical system and speckle generation . . . . . . . 53

4.3 Results . . . . . . . . . . . . . . . . . . . . . 59

4.4 Discussion . . . . . . . . . . . . . . . . . 60

$\begin{array}{ll}\text { Appendices } & 65\end{array}$

B Contrast of the time-integrated intensity for a fully dynamic speckle 67 
$\begin{array}{ll}\text { C Supplementary information } & 71\end{array}$

5 Handheld versus mounted laser speckle contrast perfusion imaging demonstrated in psoriasis lesions

5.1 Introduction . . . . . . . . . . . . . . . . . 74

5.2 Methods and materials . . . . . . . . . . . . . . . 75

5.3 Results . . . . . . . . . . . . . . . . . . . . . 84

5.4 Discussion . . . . . . . . . . . . . . . . . . . . . . 90

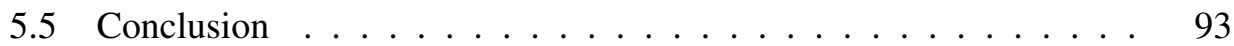

5.6 Supplementary data . . . . . . . . . . . . . . . . . 95

6 Assessment of flow within developing chicken vasculature and biofabricated vascularized tissues using multimodal imaging techniques $\quad 109$

6.1 Introduction . . . . . . . . . . . . . . . . . . . . . 110

6.2 Methods . . . . . . . . . . . . . . . . . . . . . 113

6.3 Results and discussion . . . . . . . . . . . . . . . . . . . 119

6.4 Summary . . . . . . . . . . . . . . . . . . . . . . . . . 129

6.5 Conclusion and future work . . . . . . . . . . . . . . . . 133

6.6 Supplementary figures . . . . . . . . . . . . . . . 135

7 Conclusion and outlook 145

7.1 Concluding marks . . . . . . . . . . . . . . . . . . 145

7.2 Future directions $\ldots \ldots \ldots \ldots \ldots \ldots$

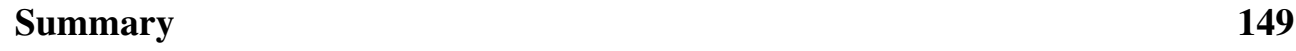

$\begin{array}{ll}\text { Samenvatting } & 151\end{array}$

$\begin{array}{ll}\text { Acknowledgements } & 153\end{array}$

About the author 155 


\section{1 \\ Introduction}

\subsection{Laser speckle contrast perfusion imaging}

Laser speckle contrast imaging (LSCI) is a noninvasive method for evaluation of microcirculatory blood flow [1]. In terms of processing time and product cost, LSCI is faster and more affordable than laser Doppler perfusion imaging (LDPI) [2], since LDPI uses an expensive and bulky high speed camera [3]. But it should also be acknowledged that LDPI facilitates direct measurement of flow velocity as well as providing a higher temporal and spectral resolution compared to LSCI [4]. The basic components of LSCI include a coherent light source and a low speed camera [5]. When being projected on the tissue, the coherent light propagates through it and interacts with static and dynamic scattering particles. When viewed with a camera, a time-variant interference pattern is observed, called speckle [6]. The contrast $C$ of a speckle pattern captured with a certain exposure time is a measure of the relative tissue perfusion: the higher the perfusion, the lower the measured contrast [7]. LSCI has a broad medical application such as reconstructive surgery [8], burns [9] and dermatology [10]. Intraoperative LSCI can provide an accurate and objective assessment of the state of vascularity of various tissues (e.g. parathyroid gland [11]) and their viability.

\subsubsection{Handheld use}

Commercially available LSCI systems are bulky and operate in mounted modality. The former limits their application in operation theatres and other clinical contexts with space limitation while the latter brings inconvenience for both patients and clinicians 


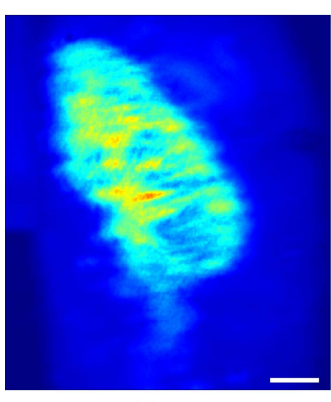

(a)

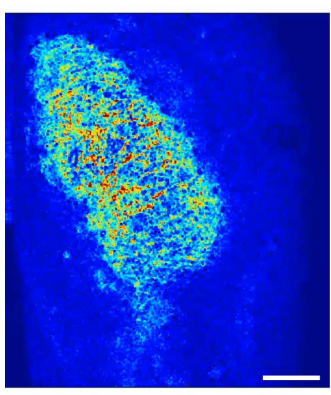

(c)

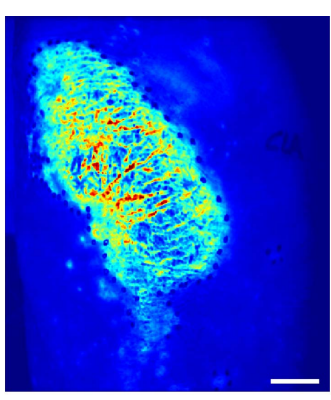

(b)

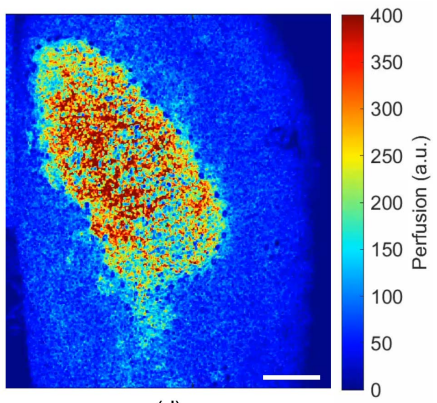

(d)

Fig. 1.1: Demonstration of movement artefacts in handheld laser speckle contrast perfusion imaging in a psoriatic lesion on a human lower arm. Temporally averaged perfusion maps acquired with a frame rate of $30 \mathrm{~Hz}$, an exposure time of $10 \mathrm{~ms}$ and a duration of 7 seconds (a) without and (b) with spatial alignment of all perfusion images before averaging. Spatial blurring is evident in (a) compared to (b). Scale bars, $2 \mathrm{~cm}$. A single perfusion map during a handheld operation with (c) low and (d) high magnitudes of applied movements. An increased level of measured perfusion is observed in (d) compared to (c) due to the movement artefacts.

[12]. Such limitations give rise to develop handheld and compact devices for LSCI. A tablet-based handheld LSCI system was proposed by Farraro et al. for point-of-care use [13]. A handheld LSCI system was developed by Rege et al. for retinal perfusion imaging [14]. An LSCI system embedded in a smart phone was shown by Kong et al. [15].

\subsubsection{Movement artefacts}

As opposed to all the benefits a handheld LSCI system offers, it can lead to unreliable measurement results due to the involuntarily movements applied during a measurement either by the patient [16] or by the operator [17]. This is due to the high level of speckle sensitivity to any source of movements. For a comparison between a typical mounted and handheld perfusion perfusion imaging see Visualization 1.1 and Visualization 1.2, respectively. When a handheld LSCI measurement is operated, movement artefacts 
show their influence in two ways: (1) spatial blurring of a temporally averaged perfusion map (see Fig. 1.1(a-b)); (2) measured higher perfusion than its actual level due to extra motion of the speckle patterns within the exposure time (see Fig. 1.1(c-d)). This thesis is outlined around the design of a handheld perfusion imager (HAPI), use of it in a clinical research setting, and study of the problem of movement artefacts in a handheld LSCI system.

\subsection{Thesis outline}

In Chapter 2 of the thesis, we investigate the relation between the speckle contrast and applied speed in both motorized and handheld scenarios, for static objects. We measure the movements of a handheld LSCI system using an electromagnetic (EM) tracking system and determine the contribution of tilt and translation of wavefronts on speckle contrast drop. We also investigate to which extent the magnitude of speckle contrast drop depends on the scattering properties of the medium.

In Chapter 3, we focus on the wavefront types in the illumination system and study the speckle contrast during motorized and handheld measurements on static objects. We consider several types of movements and explore the difference between using planar, spherical and scrambled waves. We also examine whether the incorporation of an aiming beam in the handheld system will help investigators to keep the system more stable during handheld measurements.

In Chapter 4, we develop and evaluate a theoretical model to predict speckle contrast drop due to linear motion, and for static objects. This is a first step in modelling movement artefacts due to linear motion and rotation when measuring on objects with internal motion, such as perfused tissue.

In Chapter 5, we explore the validity of handheld measurements compared to mounted measurements (as the golden standard) demonstrated in psoriatic skin. A methodology is proposed to post-process the acquired raw speckle frames under the presence of small natural movements of patients and operators, and to compute a representative perfusion map per experiment. Moreover, the theoretical model for assigning perfusion values to the measured speckle contrast is examined in-vitro and in-vivo. The aim is to study the proportionality of estimated perfusion and the applied speed.

Our work in Chapter 6 is not directly related to handheld LSCI, but investigates application of LSCI in tissue engineering. The complete superficial vasculatures of developing chicken embryos in an artificial eggshell are imaged using color imaging and LSCI. The former is used to quantify vessel properties such as diameter while the later is used to explore the blood flow level of the vasculature at different locations and times. LSCI experiments are also performed on biofabricated muscle tissues containing a perfusable channel as a proof-of-concept (POC) to show the application 
of LSCI in engineered tissues. In addition to LSCI and color imaging, a side-stream dark field (SDF) probe is used to visualize capillary structures and estimate erythrocyte velocity on several locations of the vasculature.

Finally, Chapter 7 concludes the thesis and describes possible future directions.

\section{References}

[1] L. Bento, L. Tavera, P. Assuncao, S. Faria, and R. Fonseca-Pinto, "Evaluation of cutaneous microcirculation patterns by laser speckle imaging", in 201841 st International Convention on Information and Communication Technology, Electronics and Microelectronics, MIPRO 2018 - Proceedings, 290-293 (2018).

[2] J. D. Briers, "Laser Doppler, speckle and related techniques for blood perfusion mapping and imaging", Physiological Measurement 22 (2001).

[3] M. Draijer, E. Hondebrink, T. van Leeuwen, and W. Steenbergen, "Twente Optical Perfusion Camera: system overview and performance for video rate laser Doppler perfusion imaging", Optics Express 17, 3211 (2009).

[4] A. Serov, B. Steinacher, and T. Lasser, "Full-field laser Doppler perfusion imaging and monitoring with an intelligent CMOS camera", Optics Express 13, 3681 (2005).

[5] T. M. Le, J. S. Paul, and S. H. Ong, "Laser Speckle Imaging for Blood Flow Analysis", in Computational Biology, 243-271 (2009).

[6] G. Satat, C. Barsi, and R. Raskar, "Skin perfusion photography", 2014 IEEE International Conference on Computational Photography, ICCP 2014 (2014).

[7] J. Senarathna, A. Rege, N. Li, and N. V. Thakor, "Laser speckle contrast imaging: Theory, instrumentation and applications", IEEE Reviews in Biomedical Engineering 6, 99-110 (2013).

[8] J. Zötterman, "Laser Speckle Contrast Imaging in Reconstructive Surgery", Ph.D. thesis, Linköping (2020).

[9] K. Zheng, E. Middelkoop, M. Stoop, P. van Zuijlen, and A. Pijpe, "Validity of laser speckle contrast imaging for the prediction of burn wound healing potential", Burns 1-9 (2021).

[10] P. FP, "Use of Laser-Speckle Contrast Analysis in the Study of "Non Healing" Leg UlcersA Preliminary Study", Journal of Dermatology and Cosmetology 2, 12-14 (2018).

[11] E. A. Mannoh, "Development of an Intraoperative Imaging Tool for Thyroid and Parathyroid Surgical Guidance", Ph.D. thesis (2021).

[12] B. Lertsakdadet, C. Dunn, A. Bahani, C. Crouzet, and B. Choi, "Handheld motion stabilized laser speckle imaging", Biomedical Optics Express 10, 5149 (2019). 
[13] R. Farraro, O. Fathi, and B. Choi, "Handheld, point-of-care laser speckle imaging", Journal of Biomedical Optics 21, 094001 (2016).

[14] A. Rege, S. I. Cunningham, Y. Liu, K. Raje, S. Kalarn, M. J. Brooke, L. Schocket, S. Scott, A. Shafi, L. Toledo, and O. J. Saeedi, "Noninvasive assessment of retinal blood flow using a novel handheld laser speckle contrast imager", Translational Vision Science and Technology 7 (2018).

[15] P. Kong, H. Xu, R. Li, G. Huang, and W. Liu, "Laser Speckle Contrast Imaging Based on a Mobile Phone Camera", IEEE Access 9, 76730-76737 (2021).

[16] J. Zötterman, R. Mirdell, S. Horsten, S. Farnebo, and E. Tesselaar, "Methodological concerns with laser speckle contrast imaging in clinical evaluation of microcirculation", PLoS ONE 12, 1-11 (2017).

[17] B. Lertsakdadet, B. Y. Yang, C. E. Dunn, A. Ponticorvo, C. Crouzet, N. Bernal, A. J. Durkin, and B. Choi, "Correcting for motion artifact in handheld laser speckle images", Journal of Biomedical Optics 23, 1 (2018). 


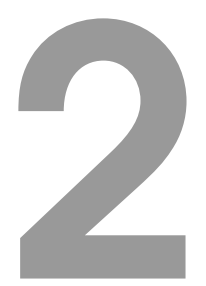

\title{
Exploration of movement artefacts in handheld laser speckle contrast perfusion imaging *
}

\begin{abstract}
Functional performance of handheld laser speckle contrast imaging (LSCI) is compromised by movement artefacts. Here we quantify the movements of a handheld LSCI system employing electromagnetic (EM) tracking and measure the applied translational, tilt and on-surface laser beam speeds. By observing speckle contrast on static objects, the magnitudes of translation and tilt of wavefronts are explored for various scattering levels of the objects. We conclude that for tissue mimicking static phantoms, on-surface speeds play a dominant role to wavefront tilt speed in creation of movement artefacts. The ratio depends on the optical properties of the phantom. Furthermore, with the same applied speed, the drop in the speckle contrast increases with decreasing reduced scattering coefficient, and hence the related movement artefact increases.
\end{abstract}

\subsection{Introduction}

As a full-field, non-invasive and affordable imaging tool, laser speckle contrast imaging (LSCI) has been widely investigated for applications such as blood flow studies in brain, neuroscience, dermatology, rheumatology and burns [1,2]. Since the conventional

\footnotetext{
${ }^{*}$ Chizari, A., Knop, T., Sirmacek, B., van der Heijden, F. and Steenbergen, W., 2020. Exploration of movement artefacts in handheld laser speckle contrast perfusion imaging. Biomedical optics express, 11(5), pp.2352-2365. https://doi.org/10.1364/BOE.387252
} 
mounted LSCI systems are bulky, development of the technology as a handheld modality has gained attention in the past few years [3-6]. A handheld camera-phone based LSCI has been used to study murine cerebral malaria by visualizing retinal perfusion [7]. In some clinical settings such as neonatal intensive care units, rapid instrumentation and compactness is of paramount interest [8]. Moreover a handheld LSCI can help comfortably monitoring any skin area of a patient, for instance when their knees or feet needed to be observed in case of having Psoriasis, burns or diabetic wounds. Beside the advantages of portability, integrability and affordability, movement artefacts pose challenges in performing an accurate and reliable measurement.

The first study on movement artefacts was carried out by Mahé et al. [9] where the backscattered light from an adjacent opaque surface and skin surface was measured. They found a linear factor of proportionality between the two signals. Then, using point by point subtraction, the movement artefact was shown to be reduced. However, the movements by patients were not applied in a standardized way. The correction should also be optimized per different test subjects. In a later work, this technique, referred to as 'a specific post-processing procedure', was employed to examine LSCI during exercise on healthy subjects [10]. Omarjee et al. [11] proposed to use a bi-layer adhesive opaque surface to detect movement-related signal and subtract it from the artefact-induced LSCI signal recorded from skin surface in an attempt to make the artefact removal procedure calibration-free. However, the movement artefacts caused by handheld measurement were not considered in that work. A handheld study by Lertsakdadet et al. [12] sorted the recorded frames based on the speckle contrast value of a fiducial marker used in the imaging protocol to filter the frames exceeding a certain threshold. Then, the selected frames were aligned based on edge-detection of a fiducial marker to decrease blurring of the resultant time-averaged contrast map. However, selecting only proper frames may cause data loss. They recently showed reduction of movement artefacts during handheld LSCI measurement thanks to the gimbal stabilizer which was at the expense of increasing total weight of the system [13].

Rather than adding an improved method for correcting movement artefacts, here we take a step back to explore the effect of movement artefacts in handheld laser speckle perfusion imaging by investigating the relation between the speckle contrast and applied speed in both motorized and handheld scenarios, for static objects. We measure the movement of a handheld LSCI system using an electromagnetic (EM) tracking system and determine the contribution of tilt and translation of wavefronts on speckle contrast drop. We also investigate to which extent the magnitude of speckle contrast drop depends on the scattering properties of the medium. 


\subsection{Methods}

\subsubsection{Handheld LSCI Probe Design}

The designed handheld probe is illustrated in Fig. 2.1(a) which has a total weight of approximately $750 \mathrm{~g}$ including the attached cables. The light source was chosen to be a continuous wave single longitudinal mode laser (CNI MSL-FN-671) of $671 \pm 1 \mathrm{~nm}$ wavelength. The output power was $300 \mathrm{~mW}$ with a coherence length of longer than $50 \mathrm{~m}$. An absorptive filter with optical density of 0.2 (Thorlabs NE02A) was mounted in front of the laser beam with some angle deviation to prevent direct reflection to the laser source. The laser beam was further directed using broadband dielectric mirrors of wavelength $400-750 \mathrm{~nm}$ (Thorlabs BB1-E02) to a microscope objective of magnification 20 (Nikon CFI Plan Fluor DIC N2) in order to make a focus to a single mode optical fiber (Thorlabs SM600) with operating wavelength of $633-780 \mathrm{~nm}$. The distal end of the optical fiber was mounted on the handheld-LSCI probe followed by a $20^{\circ}$ top hat square engineered diffuser (Thorlabs ED1-S20-MD) to form a square uniform light beam. The distance from the fiber tip to the diffuser was set to approximately $2 \mathrm{~cm}$. The backscattered light was recorded using a USB3 monochrome camera (Basler acA2040 55um) with image depth of 8 bits, frame size of $2048 \times 1536$ pixels, exposure time of $25 \mathrm{~ms}$ and operating frame rate of $40 \mathrm{~Hz}$. The camera objective (FUJINON HF16XA-5M) had a focal length of $16 \mathrm{~mm}$ and the focus range from $10 \mathrm{~cm}$. The f-number for the experiments was set to $F 8$. Based on our measurement, this is the optimum aperture size for the system in terms of speckle size to meet the Nyquist criterion [14] and in terms of detected light intensity to obtain the required dynamic range for computation of the speckle contrast. To only detect the laser light, a hard coated bandpass interference filter of wavelength $675 \pm 12.5 \mathrm{~nm}$ (Edmund Optics) was mounted in front of the camera objective. A linear polarizer optimized for the wavelengths $600-1100 \mathrm{~nm}$ (Thorlabs LPNIRE100-B) was used to suppress detection of surface reflection and increase the speckle contrast. The light beam from the source was linearly polarized and the polarization was partly lost in the single mode optical fiber and engineered diffuser. By rotating the diffuser to a certain angle at which the specular reflection was minimized, we ensured that the direction of laser light polarization is perpendicular to the employed detection polarizer.

\subsubsection{Motorized Translational-Rotational Stage}

A translation stage was driven by a DC motor (Faulhaber DC-Minimotor). This system was designed such that a range of continuous speeds of $0-10 \mathrm{~mm} / \mathrm{s}$ can be realized. In our experiments, the distance from the camera to the phantom surfaces was set to $20 \mathrm{~cm}$. A motorized precision rotation stage (Thorlabs PRM1/M28) was connected between the handheld LSCI probe and a vertical bar mounted on the translational panel. 


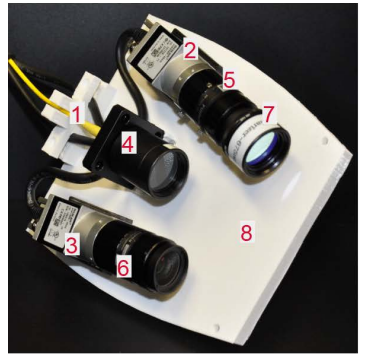

(a)

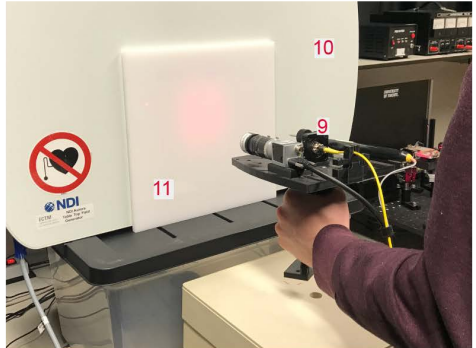

(b)

Fig. 2.1: Experimental setup. (a) Handheld LSCI system. (b) Handheld LSCI measurement and positioning. 1: optical fiber; 2: monochromatic camera; 3: color camera; 4: engineered diffuser; 5 and 6: camera objectives; 7: bandpass filter and linear polarizer; 8: panel and grip; 9: handheld LSCI system including the EM-tracker positioning sensor; 10: table-field generator EM-tracker; 11: Delrin plate.

This stage was controlled via a brushed DC servo motor unit (Thorlabs KDC101) to enable rotational speeds of up to $25 \%$ s using the software Kinesis.

\subsubsection{EM-Tracking System}

We used an NDI Aurora table top field generator as localization device [15] in order to measure the movements of the LSCI system as translational, tilt and on-surface speeds during the handheld experiments. A six degrees of freedom sensor with rootmean-square accuracy of $0.8 \mathrm{~mm}$ and $0.7^{\circ}$ respectively for position and orientation was installed on the handheld LSCI probe. Location of the probe with a rate of $40 \mathrm{~Hz}$ is sensed via inducing small currents in the sensor by altering the electromagnetic field produced by the Field Generator. The positioning accuracy depends on the distance between the sensor and the Field Generator. Based on several experiments in our setup, for a distance of $20 \mathrm{~cm}$ with the sensor placed perpendicular to the Field Generator, the highest accuracy was obtained. The output data from the system included quaternion and three-dimensional position matrices. The algorithm to convert quaternion $\left(Q_{0}, Q_{1}, Q_{2}, Q_{3}\right)$ to $(\theta, \phi, \psi)$ was written in a custom-made MATLAB R2017b program.

\subsubsection{Data Analysis}

\section{Speckle contrast}

The speckle contrast is defined as [16];

$$
C=\frac{\sigma_{s}}{\bar{I}_{s}}
$$




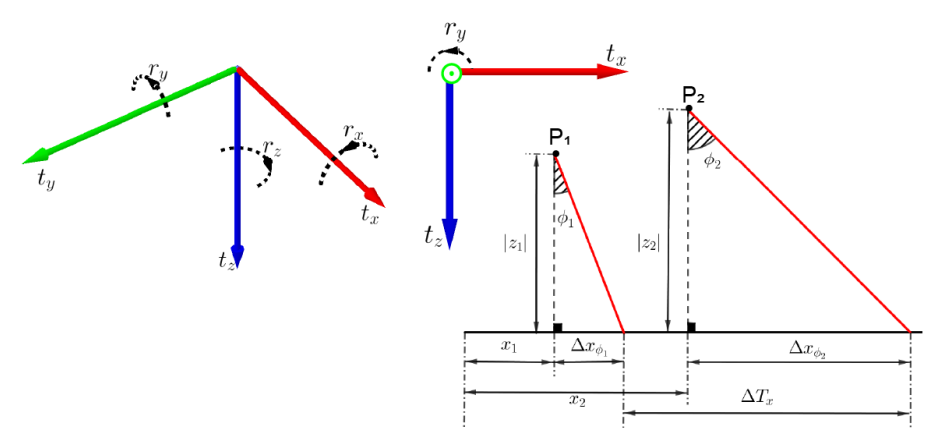

(a)

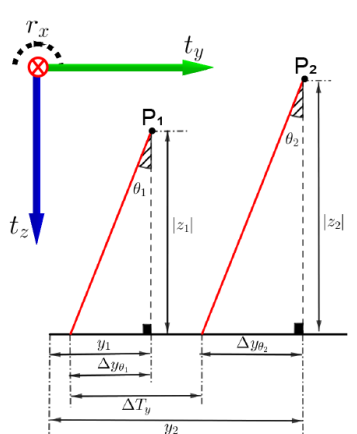

(c)

Fig. 2.2: Mapping three-dimensional (3D) movements of probe to the laser beam displacement on the surface. Schematic diagram of data analysis for six degrees of freedom motion sensor has been shown. (a) 3D coordination system defined as a set of translational and rotational vectors. Solid arrows: translational vectors; dashed curved arrows: rotational vectors; $t_{x}$ : surge; $t_{y}$ : sway; $t_{z}:$ heave; $r_{x}:$ roll; $r_{y}$ : pitch; $r_{z}$ : yaw. Movement of positioning probe during two consecutive data points $\left(P_{1}\right.$ and $\left.P_{2}\right)$ and the corresponding displacement of laser beam on $x$-direction (b) and $y$-direction (c). The pair of $\left(\Delta T_{x}, \Delta T_{y}\right)$ indicates the total displacement on the $x y$ plane.

where $\sigma_{s}$ and $\bar{I}_{s}$ represent the standard deviation and the mean values of the pixel intensities within a captured speckle frame. The speckle contrast is globally calculated for each frame; therefore, there is one speckle contrast value per frame. To obtain a decent statistical averaging, the region of interest (ROI) was chosen to be $150 \times$ 150 pixels. This ROI was selected from the center part of the camera sensor array. Since imaging and processing time were not in the scope of this work, we directly calculated the speckle contrast values of sequential frames using Eq. (2.1) and form a profile such as those shown in Fig. 2.3.

\section{Motion vector}

The algorithm of mapping the six-dimensional displacement of the probe (translations and rotations) into the two-dimensional displacements and tilts of the beam on the reference surface per two consecutive samples is described here. The purpose is to evaluate how much laser beam translated and tilted on the surface due to the applied movements in a typical handheld measurement. We first recorded the location of the sensor tip in three-dimensional space $\left(t_{x}, t_{y}, t_{z}\right)$ in millimeter per acquisition. Its instantaneous rotation along each axis $\left(r_{x}, r_{y}, r_{z}\right)$ in degrees was also the input to our algorithm (Fig. 2.2(a)). The orientation of the rotational vectors along each axis follows the right-hand rule. Assume that at times $t_{0}$ and $t_{0}+\Delta T$, the sensor is placed from location $P_{1}$ to $P_{2}$ with arbitrary rotations. We split the surface displacement of 
the beam on $x y$ plane into $x$ and $y$ vectors. On the $x$-direction (Fig. 2.2(b)), the pair of $\left(x_{1}, x_{2}\right)$ represents the locations from a reference point where the pair of $\left(z_{1}, z_{2}\right)$ corresponds to the heights at $P_{1}$ and $P_{2}$, respectively. In this case, the displacement of the beam from a perpendicular point due to the rotations can be calculated as;

$$
\left\{\begin{array}{rl}
\Delta x_{\phi_{1}} & =\left|z_{1}\right| \tan \phi_{1} \\
\Delta x_{\phi_{2}} & =\left|z_{2}\right| \tan \phi_{2}
\end{array},\right.
$$

where the pair of $\left(\phi_{1}, \phi_{2}\right)$ are the corresponding pitches. Therefore, the total beam displacement on the $x$-direction can be written as;

$$
\Delta T_{x}=x_{2}+\Delta x_{\phi_{2}}-\left(x_{1}+\Delta x_{\phi_{1}}\right) .
$$

In this way, the projected displacement on the $x$-axis is obtained taking to the account the change in (1) height; (2) location on $x$-direction; and (3) rotation around $y$-axis (pitch). Similarly, on the $y$-direction (Fig. 2.2(c)), one can write;

$$
\left\{\begin{array}{l}
\Delta y_{\theta_{1}}=\left|z_{1}\right| \tan \theta_{1} \\
\Delta y_{\theta_{2}}=\left|z_{2}\right| \tan \theta_{2}
\end{array},\right.
$$

where the pair of $\left(y_{1}, y_{2}\right)$ stands for the associated locations from a reference point and the pair of $\left(\theta_{1}, \theta_{2}\right)$ are the corresponding rolls. The total beam displacement on the $y$-direction is;

$$
\Delta T_{y}=y_{2}-\Delta y_{\theta_{2}}-\left(y_{1}-\Delta y_{\theta_{1}}\right) .
$$

Here we have taken into account the change in (1) height; (2) location on $y$-direction; and (3) rotation around $x$-axis (roll). Thus, the magnitude of surface motion vector $\left(\Delta T_{x} \vec{t}_{x}+\Delta T_{y} \vec{t}_{y}\right)$ is defined as; $\sqrt{\Delta T_{x}^{2}+\Delta T_{y}^{2}}$. This parameter times the sampling rate of the positioning device forms the temporal profile of on-surface beam speed in unit distance per unit time.

This way of calculating on-surface beam speed in a handheld measurement depends on the height and combines the influence of applied translations and rotations. Hence, to make it independent of the distance and distinguish between translational and tilt speeds, we consider the translational speed as;

$$
\frac{\sqrt{\left(x_{2}-x_{1}\right)^{2}+\left(y_{2}-y_{1}\right)^{2}}}{\Delta t} \text {. }
$$

And the instantaneous tilt angle (refers to as tilt of wavefronts) is determined as;

$$
\gamma=\tan ^{-1} \sqrt{\tan ^{2} \theta+\tan ^{2} \phi} .
$$

In a similar way, the tilt speed can be calculated by time derivation of the aforementioned tilt angle. The tilt angle is a measure of rotations along $x$ and $y$ axes which causes the wavefront tilting. The rotation around the beam axis (i.e. $z$ axis) is not considered to cause a wavefront tilt. However, it causes a nonuniform translation which is considered negligible in this work. 


\subsubsection{Handheld LSCI Measurement Protocol}

10 healthy subjects with a normal ability of holding the LSCI probe still (e.g. without hand tremor disease) participated in the study. The purpose was to measure the amount of movements in a typical handheld operation. Therefore, subjects were asked to avoid any over-concentration for reducing the movements. After the start of each measurement, the probe was mounted on the table for 15 seconds to make the baseline. Then, it was lifted slowly and kept still for $45 \mathrm{~s}$. To make sure that the time interval during which the probe was lifted was not included in the data analysis, the last $40 \mathrm{~s}$ of each measurement was accounted for the effective handheld measurement. During the handheld operation, subjects stood in front of the table top Field Generator in a relaxed manner with arm bent at elbow at $90^{\circ}$ (see Fig. 2.1(b)). To prevent metal artefacts, subjects were asked to remove any metal-made wearables. In addition, the approximate distance from the front side of the handheld probe to the Delrin's surface was kept at $20 \mathrm{~cm}$ to minimize the noise level of the EM-tracker's signal. Here, the metal artefact is referred to as interference of the electromagnetic fields caused by metal objects located close to the positioning sensor [17].

\subsubsection{In-vitro Static Phantoms}

We made four 3D printed molds with Polylactic Acid (PLA) material, each of dimensions $195 \times 60 \times 14 \mathrm{~mm}^{3}$ in which agar phantoms were cast. To make the phantom static (reduce the Brownian motion) a stock solution of demi-water with $1 \%$ agar powder (Sigma A7921-500G) was prepared. Using the spectrophotometer of wavelength $[300-1100 \mathrm{~nm}]$ (Shimadzu UV-2600) the absorption coefficient of Ecoline 700 ink (Talens) was measured as $24.6 \mathrm{~mm}^{-1}$ at the operating wavelength of $671 \mathrm{~nm}$. The Ecoline 700 was added when the stock solution had cooled down to around $60{ }^{\circ} \mathrm{C}$. We used Intralipid 20\% (Fresenius Kabi Nederland BV) for making the phantoms optically scattering. Assuming the reduced scattering coefficient of Intralipid as $26 \mathrm{~mm}^{-1}$ at the operating wavelength of $671 \mathrm{~nm}$ [18], the molds were poured with $3.7,7.7,11.5$ and 15.4 vol\%. Then, the phantoms were kept for two hours to reach room temperature. To realize a high scattering media, a black metal plate was painted with Chalk spray (Vintage) of color ultra matte. The sample called Delrin was of Polyoxymethylene material. The absorption coefficients of the agar phantoms were chosen to be the same and equal to $0.01 \mathrm{~mm}^{-1}$ while each having different reduced scattering coefficient, namely 1, 2, 3 and $4 \mathrm{~mm}^{-1}$ to cover the scattering properties of human tissue. These optical property values were adapted from Lister et al. [19] for the operating wavelength of $671 \mathrm{~nm}$. 


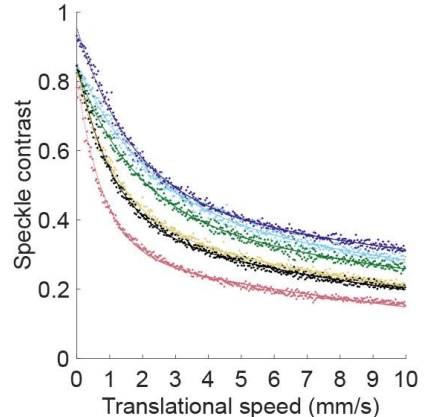

(a)

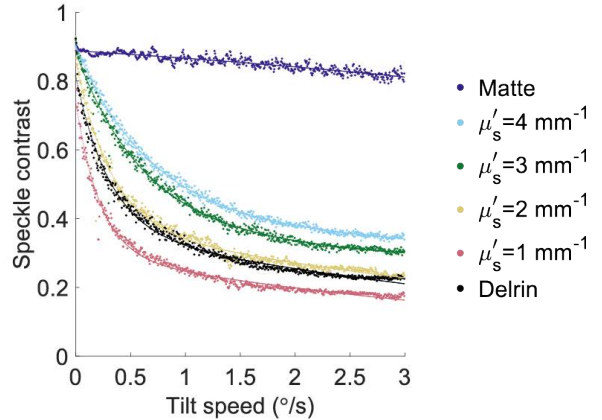

(b)

Fig. 2.3: Dependence of speckle contrast on the applied translations and tilts for various levels of scattering. (a) Speckle contrast vs. applied translational speeds. (b) Speckle contrast vs. applied tilt speeds. $\mu_{s}^{\prime}$ : reduced scattering coefficient. Solid curves: second order exponential fit functions.

\subsubsection{In-vivo Measurements}

Translational speeds from 0 to $10 \mathrm{~mm} / \mathrm{s}$ in $3.3 \mathrm{~s}$ were applied to the LSCI system and the speckle contrast was measured on a window of $150 \times 150$ pixels with the system facing on the forearms of test subjects. The distance from the camera sensor to the skin surface was set to $20 \mathrm{~cm}$. The camera operated at $40 \mathrm{~Hz}$ acquisition rate with an exposure time of $25 \mathrm{~ms}$ and $0 \mathrm{~dB}$ gain. Two healthy test subjects participated in the study including 2 phases of (1) measuring on a skin area on the forearm with normal perfusion level and (2) 15 minutes after application of $0.2 \mathrm{ml}$ vasodilating cream (60 gr Midalgan cream extra warm, Qualiphar, Meppel, The Netherlands) on an area of $20 \times 5 \mathrm{~cm}^{2}$. Each phase consisted of a $3 \mathrm{~s}$ baseline measurement during which the LSCI system was kept still. Then the system started to move along the forearm. Subjects were asked to breath normally and to keep their arm still during the measurements.

\subsection{Results}

\subsubsection{Speckle Contrast due to Controlled Translation and Tilting for Various Scattering Properties}

To investigate the contribution of translation and tilt of wavefronts on the speckle contrast drop, two independent experiments were designed. For each experiment, static phantoms of various optical properties were used as diffuse media. The speckle size for this dataset was measured as $3 \times 3$ pixels. With a choice of ROI size of $150 \times 150$ pixels, there will be around 2500 samples per frame which leads to a 
statistically reliable calculation of speckle contrast.

For the translation study, the probe was faced perpendicular to the object surface and linear translational speed was applied to the system. Figure 2.3(a) depicts the measured speckle contrast in terms of the applied translational speeds. For the same applied speed, the less scattering media tend to cause larger drop in the speckle contrast. Visualization 2.1, Visualization 2.2 and Visualization 2.3 illustrate speckle patterns and the corresponding contrast versus applied speed for matte, Delrin and the phantom of $\mu_{s}^{\prime}=1 \mathrm{~mm}^{-1}$, respectively. For rotation, the LSCI system was mounted still and the phantom was rotated around a vertical axis with a constant acceleration (Fig. 2.3(b)). Since the center of rotation was aligned to the phantom surface, the effect of tilt of wavefronts was taken into account without translation. Similarly, for the same applied tilt speed, the less scattering media tend to cause larger drop in the speckle contrast. Visualization 2.4, Visualization 2.5 and Visualization 2.6 illustrate speckle patterns and the corresponding contrast versus applied tilt speed for matte, Delrin and the phantom of $\mu_{s}^{\prime}=1 \mathrm{~mm}^{-1}$, respectively.

\subsubsection{Characterization of Movements of Handheld LSCI System}

During a handheld measurement, the movements of the LSCI system can be described as a combination of pure translations and pure rotations which causes translations and tilts of wavefronts with respect to the scattering surface. The translation of the beam on the level of the medium's surface will be referred to as on-surface speed. The on-surface speed is calculated by time derivation of beam positions on the scattering surface in which the system translation, rotation, and distance of the system to the medium play a role. The tilt speed accounts for tilting of wavefronts which is calculated as time derivation of the angle at which the handheld system is pointing with respect to the normal to the surface. To obtain an estimation of these parameters, several handheld measurements by various healthy test subjects were carried out.

Figure 2.4(a) is a so-called Lissajous graph of a representative handheld measurement on the $x y$ plane. This is an estimation for displacement of the light beam on a surface in which three episodes are of interest. (1) The baseline episode around the origin during which the system is mounted (red circle); (2) the episode where the system is being lifted (red arrow); and (3) the effective handheld measurement episode (black square). This graph shows an almost $15 \mathrm{~mm}$ and $20 \mathrm{~mm}$ total displacements on horizontal and vertical directions, respectively. Visualization 2.7 demonstrates a representative handheld measurement including a progressive plot of on-surface speed and speckle contrast. For the baseline area, the standard deviation values of the location signal fluctuations are $\sigma_{x}=7.6 \mu \mathrm{m}, \sigma_{y}=8.7 \mu \mathrm{m}$ and $\sigma_{z}=9.9 \mu \mathrm{m}$. Figure 2.4(b) depicts the total displacement of on-surface locations in time domain relative to the starting position. The absolute values of the Fourier transform of on-surface locations for baseline (noise) and effective handheld (signal) is shown in Fig. 2.4(c). 


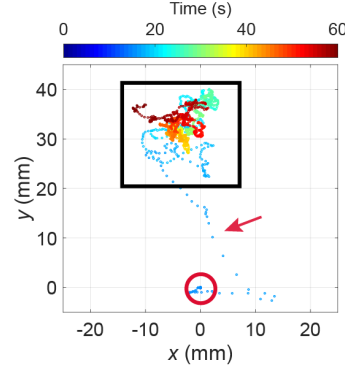

(a)

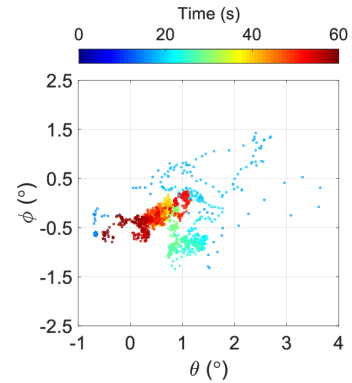

(d)

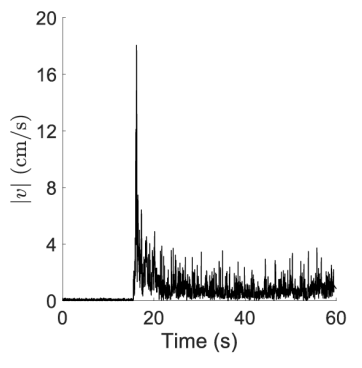

(g)

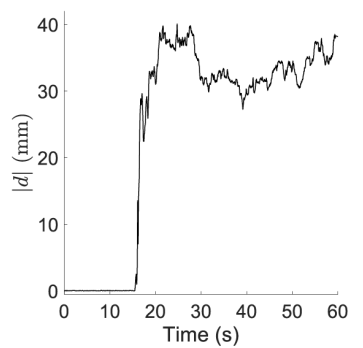

(b)

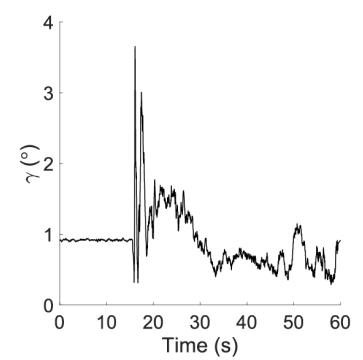

(e)

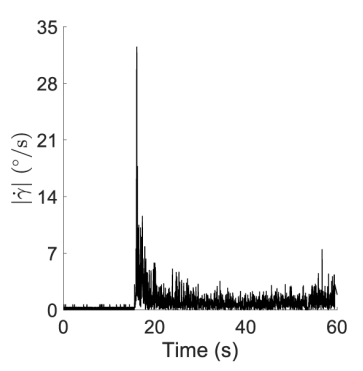

(h)

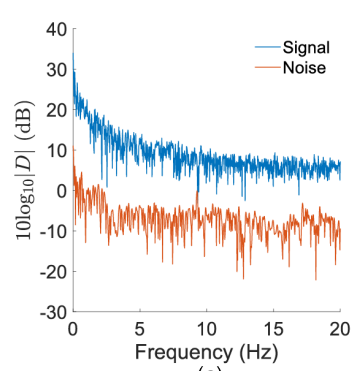

(c)

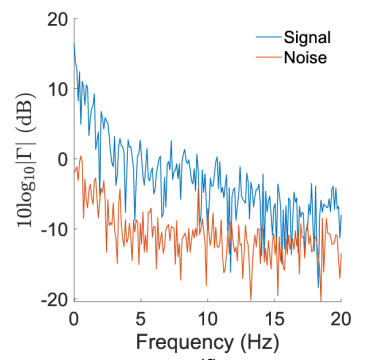

(f)

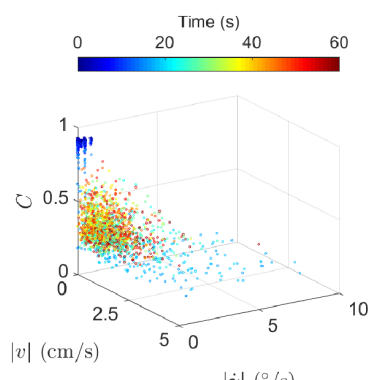

(i)

Fig. 2.4: Analysis of movement and speed of handheld LSCI system. Representative data of a handheld operation is shown. (a) Lissajous plot indicating the locations of the light beam on a scattering surface. Red circle: baseline measurement while the system is mounted; Red arrow: the episode during which the system is lifted; Black square: the effective handheld measurement. (b) Temporal fluctuations of on-surface locations. (c) Absolute Fourier transform of on-surface locations. Signal: effective handheld measurement; Noise: baseline measurement. (d) Lissajous plot of rotations along $x$ and $y$ axes shown as $\theta$ and $\phi$, respectively. (e) Temporal fluctuations of tilt angle. (f) Absolute Fourier transform of tilt angle. Temporal profiles of absolute on-surface and tilt speeds. $v$ : on-surface speed; $\dot{\gamma}$ : tilt speed. (i) Observed speckle contrast on a Delrin plate as a function of on-surface and tilt speeds. $C$ : spatial speckle contrast. (g) and (h) are still images of Visualization 2.7. (i) is still image of Visualization 2.8. 


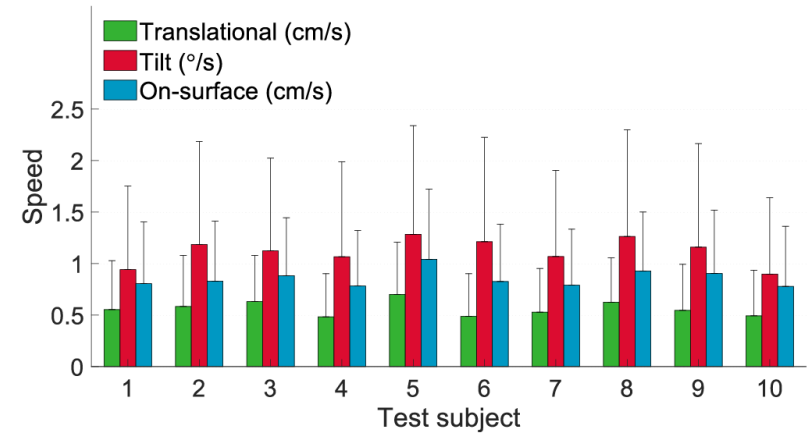

Fig. 2.5: Overview of averaged speeds estimated from handheld measurements per test subject representing the translational, tilt and on-surface speeds of the light beam. Data are mean \pm standard deviation.

The rotations of the system along $x$ and $y$ axes are shown as a Lissajous plot in Fig. 2.4(d) which is color coded with time. Based on Eq. (2.7) the instantaneous tilt angle is calculated and depicted in Fig. 2.4(e). The absolute values of the Fourier transform of tilt angle for baseline and effective handheld measurements is also illustrated in Fig. 2.4(f). For the baseline area, the standard deviation of three dimensional angles are $\sigma_{\theta}=6.5 \mathrm{~m}^{\circ}, \sigma_{\phi}=13.1 \mathrm{~m}^{\circ}$ and $\sigma_{\psi}=12.6 \mathrm{~m}^{\circ}$.

The absolute on-surface speed is defined as the absolute value of time derivative of on-surface location vectors and is shown in Fig. 2.4(g). The root mean square (RMS) value of signal to noise ratio (SNR) for this measurement is $21.5 \mathrm{~dB}$, with the signals in time intervals $0-10 \mathrm{~s}$ and $20-60 \mathrm{~s}$ are considered as noise and signal, respectively. The absolute tilt speed is also obtained by time derivation of tilt angles with an SNR of $14.5 \mathrm{~dB}$ (Fig. 2.4(h)). The observed speckle contrast as a function of on-surface and tilt speeds for a sample handheld measurement is shown in Fig. 2.4(i) (see Visualization 2.8 for a better view on this graph). The average values of extracted speed elements have been summarized in Fig. 2.5 where the mean values for all 10 operators for translational, tilt and on-surface speeds are $0.6 \mathrm{~cm} / \mathrm{s}, 1.1 \% \mathrm{~s}$ and $0.9 \mathrm{~cm} / \mathrm{s}$, respectively.

Table 2.1 summarizes the relative drop in speckle contrast due to translation and tilt of wavefronts. The values are extracted from Fig. 2.3 with two data points from each phantom: the speckle contrast at speed zero and the speckle contrast at on-surface and tilt speeds of $0.9 \mathrm{~cm} / \mathrm{s}$ and $1.1 \%$, respectively. These on-surface and tilt speed values are the averaged values of 10 handheld measurements estimated by the EM-tracker (Fig. 2.5). 


\subsubsection{In-vivo Measurements}

Temporal fluctuation of speckle contrast measured on the forearm of the first test subject after the application of Midalgan is shown in Fig. 2.6(a). The sudden drops in the speckle contrast are due to the heartbeats which occur approximately every second. The average heart rate based on these time intervals for this test subject is calculated as 64 beats per minute (bpm). In this graph, the system starts to move at the time $4.8 \mathrm{~s}$. A comparison between the observed speckle contrast vs the applied translational speed before and after application of Midalgan is shown in Fig. 2.6(b-c) for the first and the second test subjects, respectively. Here the skin area was approximately the same for each test subject and also the perfusion of the measured area is approximately the same at the start and the end locations. The speckle contrast level at zero speed after application of Midalgan is lower than that of normal perfusion level and this is the case for both test subjects. Moreover, the two graphs are not identical to each other.

\subsection{Discussion}

Movement artefacts during a handheld LSCI measurement are caused by tissue motions [9, 11, 20-23] and motions of the LSCI system [5, 12, 13]. The former can be caused by breathing or patient movements while the latter are generated in the wrist, elbow and shoulder, and motions due to heartbeat and breathing of the operator. In this work, we focused on the movement of the handheld LSCI system, and the overall motions which cause movement artefacts are considered as on-surface translational motions of the laser beam and tilt of wavefronts.

To generate controlled motions, the LSCI probe was installed on a motorized stage and the speckle contrast was measured during the applied movements. The experiments were carried out on static objects in order to exclude any additional speckle decorrelation source other than the applied external motions. The influence of optical properties of the medium on the speckle contrast was investigated for

Table 2.1: Contribution of translation and tilt of wavefronts in speckle contrast drop for various static phantoms. $\mu_{s}^{\prime}$, reduced scattering coefficient; $\% \Delta C_{x}$, speckle contrast drop percentage; on-surf., onsurface speed; tilt., tilt speed.

\begin{tabular}{l|cccccc}
$\mu_{s}^{\prime}\left(\mathrm{mm}^{-1}\right)$ & $>4($ Matte $)$ & 4 & 3 & 2 & $\approx 2($ Delrin $)$ & 1 \\
\hline$\Delta C_{\text {on-surf. }}(\%)$ & 63.4 & 63.9 & 67.1 & 71.9 & 72.6 & 78.2 \\
\hline$\Delta C_{\text {tilt. }}(\%)$ & 5.5 & 46.3 & 53.6 & 59.6 & 63.2 & 70.3 \\
\hline \hline$\Delta C_{\text {on-surf. }} / \Delta C_{\text {tilt. }}$ & 11.6 & 1.4 & 1.2 & 1.2 & 1.1 & 1.1
\end{tabular}




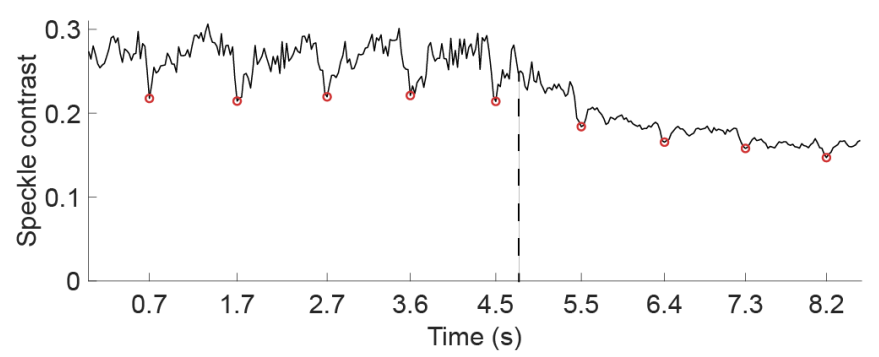

(a)

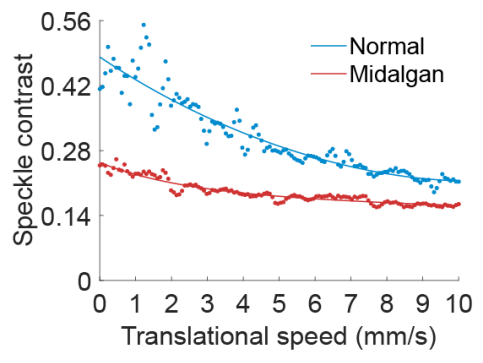

(b)

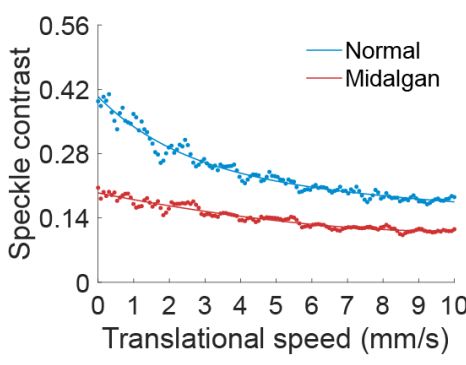

(c)

Fig. 2.6: In-vivo measurements of speckle contrast. (a) Temporal fluctuation of speckle contrast driven by heartbeat pattern. Red open circles indicate manually chosen sudden drops. During the first $4.8 \mathrm{~s}$, the LSCI system is still. The vertical dashed line indicates the moment the system starts to move. (b-c) Speckle contrast vs translational speeds for test subjects 1 and 2, respectively. 'Normal' refers to the skin area with normal perfusion level. 'Midalgan' refers to same skin area after 15 minutes application of Midalgan. Solid curves in (b-c): second order exponential fit functions.

translation and tilting. For a given absorption level, we observed (Fig. 2.3) that both for translation and tilt, the medium of less scattering properties causes greater drop in the measured speckle contrast values. Hence, tissues with lower scattering levels will generate higher movement artefacts than tissues with higher scattering levels. These findings can be explained with the use of the optical Doppler shifts on a single position on the imaging plane (the camera array sensor). Here we use the fact that intensity fluctuations have frequencies equal to the differences of Doppler shifts of the incoming light, since two light beams with Doppler frequencies $\omega_{1}$ and $\omega_{2}$ on interference give an intensity fluctuation at beat frequency $\left|\omega_{1}-\omega_{2}\right|$. And higher frequencies of the intensity fluctuations lead to more speckle blurring on integration in a finite nonzero integration time, hence a lower contrast. So we need to search for the effect of the scattering level on the differences in Doppler shifts in the complete 'ensemble' of light imaged in a single point.

For both translation and tilt of wavefronts, these differences in optical Doppler 
shifts will increase with decreasing scattering level of the medium. Fig. 2.7(a) shows schematically the case of translation where the light source and the imaging system move at a direction parallel to the object plane with speed $\vec{v}=\left(v_{x}, v_{y}, v_{z}\right)$. The reduced scattering coefficient of medium 1 is greater than that of medium 2 , that is $\mu_{s_{1}}^{\prime}>\mu_{s_{2}}^{\prime}$. Therefore, the size of the diffuse cloud of light detected at the center pixel for medium 1 (yellow fluence distribution) is smaller than that of medium 2 (red fluence distribution, partly overlapping with the yellow fluence distribution). In other words, for a given position of the diffuser in the illumination system, medium 2 causes larger variation of the incoming wave vectors $\vec{k}_{i}=\left(k_{i_{x}}, k_{i_{y}}, k_{i_{z}}\right)$ since light imaged to a single point originates from light injected into the medium over a larger area. For both medium 1 and medium 2 , the wave vectors of the detected light $\vec{k}_{s}=\left(k_{s_{x}}, k_{s_{y}}, k_{s_{z}}\right)$ are the same since they are defined by the detection lens aperture. For solid body translation the Doppler shift is [24]

$$
\omega=\vec{v} \cdot\left(\vec{k}_{s}-\vec{k}_{i}\right),
$$

and hence does not depend on the photon paths inside the medium: it is only the incoming and outgoing wave vectors that contribute to the Doppler shift. The greater the variation of incoming wave vectors $\vec{k}_{i}$ of the light that is eventually detected, the greater the frequencies of intensity fluctuations, leading to a lower speckle contrast. And this is the case for the lower scattering level, since the variation in $\vec{k}_{i}$ is larger for the wider diffuse light distribution associated with lower scattering media. We explore the consequences for a diffuser at $200 \mathrm{~mm}$ from the medium's surface. For the difference in $\vec{k}_{i}$ for light originating from a single point in the diffuser but entering the medium at $0.5 \mathrm{~mm}$ left or right from the point on the tissue surface conjugated with the point on the imaging plane, using Eq. (2.8) we find a difference in Doppler shifts of approximately $10 \mathrm{~Hz}$ per $\mathrm{mm} / \mathrm{s}$ of translational speed, leading to intensity fluctuations of $10 \mathrm{~Hz}$ per mm/s of speed. For the applied integration time of $25 \mathrm{~ms}$ an intensity fluctuation at this will lead to a significant drop in speckle contrast. Hence, our explanation makes it credible that, for a given translational speed, scattering level variations leading to variations in the dimensions of the 'diffuse cloud' in the millimeter range lead to significant variations of the speckle contrast. This makes our Doppler based explanation a plausible one.

A similar statement can be made for the case of tilt of wavefronts in the experiment of object rotation demonstrated in Fig. 2.7(b), although in that case Eq. (2.8) does not hold, and the Doppler shift will depend on the details of the photon paths. The Doppler shift will be larger for light entering the medium further away from the point of detection, and for light penetrating deeper into the medium before returning. For tilting, the Doppler shift of a given photon path now is the result of a variation of the optical path length through air plus the Doppler shift built up on scattering events in the medium. Also here the argument holds that a lower scattering leads to a larger variation of Doppler shifts, hence higher intensity fluctuation frequencies, and hence 


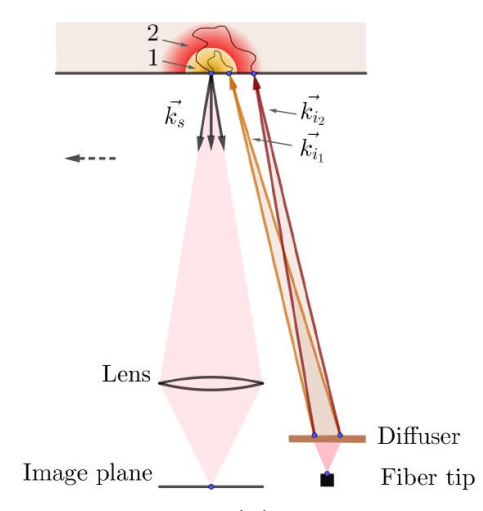

(a)

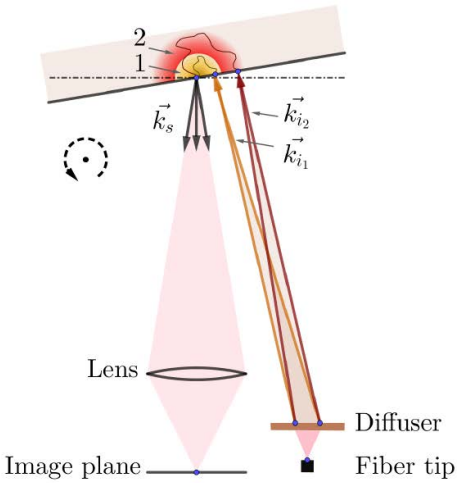

(b)

Fig. 2.7: Schematic diagram of wave vectors in a reflection geometry. (a) Translational displacement. (b) Object rotation. 1: Diffuse distribution of light eventually imaged in a single point on the image plane, for medium of higher scattering level; 2 : Idem, medium of lower scattering level; $\overrightarrow{k_{1}}$ and $\overrightarrow{k_{i_{2}}}$ illustrate wave vectors of incoming beams in 1 and 2 , respectively. $\vec{k}_{s}$ : wave vectors of light to be imaged on a single point of the image plane. The curved solid lines in the fluence distributions show random photon paths. The dashed arrows show the movement directions.

a lower speckle contrast. An extreme case is that of a very high scattering medium such as a thin layer of matte paint. Here the speckle contrast tends to be considerably less influenced by the applied tilt speed (see Fig. 2.3(b)) in comparison to the other phantoms. In this case, the speckle patterns are mainly formed by reflection of a rough surface due to the fact that the thickness of the painted surface is a couple of microns which is comparable with the wavelength of the light source. Hence light falling on a certain location of the camera sensor is originating from locally impinging light in the conjugated point on the tissue, without sideward diffusion.

We employed an EM-tracker to estimate movements of a handheld LSCI system. During the handheld measurements, the metal objects were avoided to be in the range of the electromagnetic field in order to prevent metal artefacts. To assess the accuracy and precision of this system, we first measured the discretized errors of position and angle dataset as $10 \mu \mathrm{m}$ and $11 \mathrm{~m}^{\circ}$, respectively. The discretized error is referred to as uniform steps we observed in our raw position and angle dataset which is not necessarily the minimum nonzero difference between two consecutive data points. One of the sources of this error is analog to digital conversion of the acquired signals. Then, to check the stability of raw signals, the standard deviations of location $\left(\sigma_{x}=7.6 \mu \mathrm{m}, \sigma_{y}=8.7 \mu \mathrm{m}\right.$ and $\left.\sigma_{z}=9.9 \mu \mathrm{m}\right)$ and angle $\left(\sigma_{\theta}=6.5 \mathrm{~m}^{\circ}\right.$, $\sigma_{\phi}=13.1 \mathrm{~m}^{\circ}$ and $\sigma_{\psi}=12.6 \mathrm{~m}^{\circ}$ ) data for the baseline episode were determined. Second, we presented the spectra of estimated on-surface locations and tilt angles 
(Fig. 2.4(c,f)). In both graphs, the effective handheld data have greater values than the baselines. The spectra of on-surface locations gradually drops to approximately $20 \mathrm{~dB}$ of its maximum value at the frequency of $10 \mathrm{~Hz}$ and then levels off. The frequency at which the tilt angles reach a $20 \mathrm{~dB}$ decay and remain at the same level is approximately $15 \mathrm{~Hz}$. Third, we calculated the SNR of RMS on-surface $(21.5 \mathrm{~dB})$ and tilt $(14.5 \mathrm{~dB})$ speeds (Fig. 2.4(g,h)). Note that the time derivative per two consecutive samples increases the noise of speed data rather than that of instantaneous locations and angles.

Worth noting that speckle contrasts at speed zero per each phantom shown in Fig. 2.3 are not exactly the same. This might be due to mode-hopping of the light source, external sources of vibrations or the fact that observed speckle patterns are not fully developed in practice. However, this issue does not affect the results since the relative speckle contrast is considered. The ratios of two contrast drop percentages suggest that for typical handheld motions (1) translation of the beam on the surface of the medium plays a dominant role over wavefront tilting in decreasing speckle contrast, and (2) the dominance of translation over tilt tends to decrease as the medium becomes less scattering.

We have included the in-vivo measurements in order to have a practical view on the issue of movement artefacts. Due to the movements of the red blood cells (RBCs) in the microcirculatory blood vessels, the speckle contrast values at zero speed are lower than that of static objects. Fig. 2.6(a) shows a temporal speckle contrast profile driven by heart beat pattern. The dynamic range of the fluctuations tends to decrease as the speed increases demonstrating the underlying non-linear response of the speckle contrast to the applied speed depicted in Fig. 2.3(a). The average relative speckle contrast dips at systole when the system is still is calculated as 0.07 , whereas after the system starts to move, they become $0.06,0.03,0.02$ and 0.02 , where the lower values correspond to the higher speeds. The speckle contrast drop percentages from the zero speed to that of at $0.9 \mathrm{~cm} / \mathrm{s}$ are $54.2 \%$ and $36 \%$ before and after application of Midalgan, respectively, for the first test subject shown in Fig. 2.6(b). These values for the second test subject shown in Fig. 2.6(c) are respectively 55\% and 42.1\%. This means that for the same applied speed, the relative change in the speckle contrast decrease as the skin perfusion increases and these relative changes vary per subject due to the difference in perfusion levels.

In conclusion, the magnitude of movement artefacts depends on the optical properties of the medium. We have explicitly shown that movement artefacts increase with decreasing scattering coefficient and based on our explanation it is to be expected that movement artefacts will also increase with decreasing absorption. This means that in a handheld LSCI measurement, movement artefact correction based on a simple look-up table approach is not feasible without knowledge of the applied translations and tilts and also optical properties of the tissue. The results of this study can be used for future development of strategies to suppress movement artefacts. Our findings enable 
the formulation of specifications on the performance of suppression or correction methods, be it based on hardware or software methods, since this study provides values of wavefront tilt and beam translation speeds and their temporal dynamics, and their consequences for various types of tissue.

\section{Acknowledgments}

We are grateful to Yoeko Mak and technical staff at Robotics and Mechatronics for help to build the localization setup.

\section{References}

[1] D. A. Boas and A. K. Dunn, "Laser speckle contrast imaging in biomedical optics", Journal of Biomedical Optics 15, 011109 (2010).

[2] W. Heeman, W. Steenbergen, G. M. van Dam, and E. C. Boerma, "Clinical applications of laser speckle contrast imaging: a review”, Journal of Biomedical Optics 24, 1 (2019).

[3] D. Jakovels, I. Saknite, G. Krievina, J. Zaharans, and J. Spigulis, "Mobile phone based laser speckle contrast imager for assessment of skin blood flow", in Eighth International Conference on Advanced Optical Materials and Devices (AOMD-8), edited by J. Spigulis (SPIE) (2014).

[4] D. Jakovels, I. Saknite, and J. Spigulis, "Implementation of laser speckle contrast analysis as connection kit for mobile phone for assessment of skin blood flow", in Biophotonics: Photonic Solutions for Better Health Care IV, edited by J. Popp, V. V. Tuchin, D. L. Matthews, F. S. Pavone, and P. Garside (SPIE) (2014).

[5] A. Rege, S. I. Cunningham, Y. Liu, K. Raje, S. Kalarn, M. J. Brooke, L. Schocket, S. Scott, A. Shafi, L. Toledo, and O. J. Saeedi, "Noninvasive assessment of retinal blood flow using a novel handheld laser speckle contrast imager", Translational Vision Science \& Technology 7, 7 (2018).

[6] A. Rege, M. J. Brooke, K. Murari, and Y. Liu, "System and method for rapid examination of vasculature and particulate flow using laser speckle contrast imaging”, (2018), uS Patent App. 15/767,057.

[7] I. Remer, L. F. Pierre-Destine, D. Tay, L. M. Golightly, and A. Bilenca, "In vivo noninvasive visualization of retinal perfusion dysfunction in murine cerebral malaria by camera-phone laser speckle imaging”, Journal of Biophotonics 12, e201800098 (2018).

[8] R. Farraro, O. Fathi, and B. Choi, "Handheld, point-of-care laser speckle imaging", Journal of Biomedical Optics 21, 094001 (2016).

[9] G. Mahé, P. Rousseau, S. Durand, S. Bricq, G. Leftheriotis, and P. Abraham, "Laser speckle contrast imaging accurately measures blood flow over moving skin surfaces", Microvascular Research 81, 183-188 (2011). 
[10] G. Mahé, P. Abraham, A. L. Faucheur, A. Bruneau, A. Humeau-Heurtier, and S. Durand, "Cutaneous microvascular functional assessment during exercise: a novel approach using laser speckle contrast imaging", Pflugers Archiv - European Journal of Physiology 465, 451-458 (2013).

[11] L. Omarjee, I. Signolet, A. Humeau-Heutier, L. Martin, D. Henrion, and P. Abraham, "Optimisation of movement detection and artifact removal during laser speckle contrast imaging", Microvascular Research 97, 75-80 (2015).

[12] B. Lertsakdadet, B. Y. Yang, C. E. Dunn, A. Ponticorvo, C. Crouzet, N. Bernal, A. J. Durkin, and B. Choi, "Correcting for motion artifact in handheld laser speckle images", Journal of Biomedical Optics 23, 1 (2018).

[13] B. Lertsakdadet, C. Dunn, A. Bahani, C. Crouzet, and B. Choi, "Handheld motion stabilized laser speckle imaging”, Biomedical Optics Express 10, 5149 (2019).

[14] S. J. Kirkpatrick, D. D. Duncan, and E. M. Wells-Gray, "Detrimental effects of specklepixel size matching in laser speckle contrast imaging", Optics Letters 33, 2886 (2008).

[15] Aurora, "Electromagnetic tracking system specifications", Technical Report, NDI (2013).

[16] J. W. Goodman, Speckle phenomena in optics: theory and applications (Roberts and Company Publishers) (2007).

[17] Y. Fong, P. C. Giulianotti, J. Lewis, B. G. Koerkamp, and T. Reiner, Imaging and visualization in the modern operating room, volume 112 (Springer) (2015).

[18] R. Michels, F. Foschum, and A. Kienle, “Optical properties of fat emulsions", Optics Express 16, 5907 (2008).

[19] T. Lister, P. A. Wright, and P. H. Chappell, “Optical properties of human skin”, Journal of Biomedical Optics 17, 0909011 (2012).

[20] L. M. Richards, E. L. Towle, D. J. Fox, and A. K. Dunn, "Intraoperative laser speckle contrast imaging with retrospective motion correction for quantitative assessment of cerebral blood flow", Neurophotonics 1, 015006 (2014).

[21] S. Bahadori, T. Immins, and T. W. Wainwright, "A novel approach to overcome movement artifact when using a laser speckle contrast imaging system for alternating speeds of blood microcirculation", Journal of Visualized Experiments 1 (2017).

[22] J. Zotterman, R. Mirdell, S. Horsten, S. Farnebo, and E. Tesselaar, "Methodological concerns with laser speckle contrast imaging in clinical evaluation of microcirculation", PLOS ONE 12, e0174703 (2017).

[23] S. C. Gnyawali, K. Blum, D. Pal, S. Ghatak, S. Khanna, S. Roy, and C. K. Sen, "Retooling laser speckle contrast analysis algorithm to enhance non-invasive high resolution laser speckle functional imaging of cutaneous microcirculation", Scientific Reports 7 (2017).

[24] J. Abbiss, T. Chubb, and E. Pike, "Laser doppler anemometry”, Optics \& Laser Technology 6, 249-261 (1974). 


\section{Influence of wavefront types on movement artefacts in handheld laser speckle contrast perfusion imaging}

Laser speckle contrast imaging (LSCI) is a non-invasive and affordable technique to visualize skin perfusion. Handheld use of the system facilitates measurements on various skin areas in a flexible manner. However, movement artefacts caused by handheld operation or test subject movements hamper its performance. In this work, we study the influence of the laser beam type in handheld-LSCI by evaluating the speckle contrast on static objects for beams with planar, spherical or scrambled wavefronts, and for movement artefacts caused by tilting or translation of wavefronts. We show that the scrambled waves made by often-used engineered diffusers lead to significantly larger movement artefacts than planar or spherical waves.

\subsection{Introduction}

Laser speckle contrast imaging (LSCI) is a well-known technique to study cutaneous blood flow [1]. The skin is illuminated with coherent light and the backscattered light forms a so-called speckle pattern on the imaging sensor array. Due to the interaction of light with moving red blood cells (RBCs) within the capillaries, the speckle patterns

\footnotetext{
${ }^{*}$ Chizari, A. ${ }^{+}$, Knop, T. ${ }^{+}$, Tsong, W., Schwieters, S. and Steenbergen, W., 2021. Influence of wavefront types on movement artefacts in handheld laser speckle contrast perfusion imaging. OSA Continuum, 4(6), pp.1875-1888. ${ }^{+}$These authors contributed equally. https://doi.org/10.1364/OSAC.420479.
} 
become time dependent. Different flows of RBCs will cause different blurring levels of the time integrated speckle patterns. The parameter speckle contrast is used to evaluate the actual flow [2-5]. The sensitivity of speckle patterns to small movements necessitates that during the measurement, no other source of movements should exist in order to form a reliable perfusion map. Therefore, practical realization of such experimental environment remains a challenge.

Some applications of LSCI include dermatology, burns and the diabetic foot $[6,7]$. It would be ideal to have a compact and handheld LSCI in order to operate measurements on various patient body areas without inconvenience for patients or investigators [8]. From one side, patient movements originated by breathing, heartbeat and organ tremor is a source of movement artefacts [9, 10]. From another side, when using handheld, operator-generated movements of the LSCI system caused by the operator are another source of movement artefacts. Several approaches have been proposed for decreasing movement artefacts. To measure movement related signals (i.e. speckle contrast or perfusion unit) opaque static objects have been placed in the imaging field-of-view (FOV) [11-13]. In this way, the measured signal generated by the object is only influenced by the applied handheld movements, which enables identification and potentially removal of these artefacts. It has been shown that using a motorized gimbal stabilizer, handheld-LSCI can be realized which is less influenced by movement artefacts [14]. To obtain a less noisy perfusion map, multiple perfusion maps are temporally averaged. In handheld measurements, these perfusion maps are often co-registered by segmentation or edge detection of natural textures of samples [15] or the static objects within the FOV.

As the quality of the illumination and the imaging systems directly influences the quality of LSCI measurements, the influence of the spectral width of the light source on the speckle contrast has been investigated[16]. A yet unexplored issue is the influence of the way in which the laser beam is formed. In this work, we focus on the wavefront types in the illumination system and study the speckle contrast during motorized and handheld measurements on static objects. We consider several types of movements and explore the difference between using planar, spherical and scrambled waves. We also examine whether the incorporation of an aiming beam in the handheld system will help investigators to keep the system more stable during handheld measurements. The dataset for motorized and handheld measurements can be found in Dataset 1 [17].

\subsection{Methods and materials}

\subsubsection{Handheld LSCI system}

A continuous wave single longitudinal mode laser (CNI MSL-FN-671) of $671 \pm 1 \mathrm{~nm}$ wavelength with the output power of $300 \mathrm{~mW}$ and a coherence length of longer 


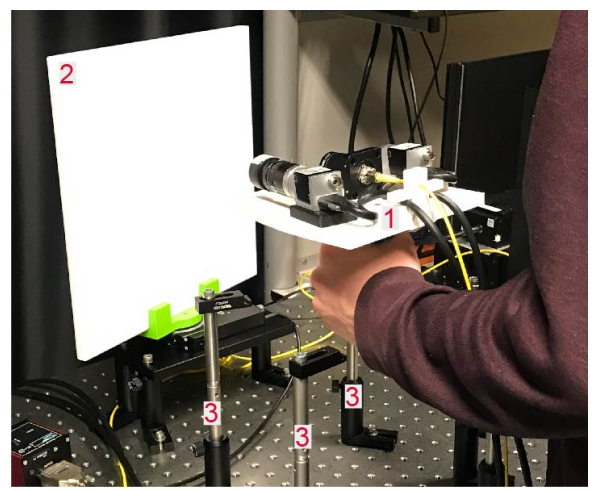

Fig. 3.1: Experimental setup for the handheld measurements. 1: Handheld LSCI system; 2: Delrin plate; 3: Mount platform for the baseline measurements.

than $50 \mathrm{~m}$ was chosen as the light source. Its output light was directed through an absorptive filter with optical density of 0.2 (Thorlabs NE02A) which was placed nonperpendicularly to the beam to prevent direct reflection to the laser tube. Broadband dielectric mirrors of wavelength $400-750 \mathrm{~nm}$ (Thorlabs BB1-E02) were used to direct the laser beam into a microscope objective of magnification 20 (Nikon CFI Plan Fluor DIC N2) mounted on a three axis stage (Thorlabs Nanomax 300) in order to focus the light into a single mode fiber (Thorlabs SM600) with operating wavelength of $633-780 \mathrm{~nm}$ and a cladding diameter of 125 micron. The distal end of the fiber was mounted on the probe. The choice of the single mode fiber was to prevent speckle change due to the movements of handheld-LSCI probe. A monochrome camera (Basler acA2040 55um USB3) was mounted on the probe to record the speckle intensity maps with a frame rate of $40 \mathrm{~Hz}$, exposure time of $10 \mathrm{~ms}$, imaging depth of 8 bits, pixel size of $3.45 \mu \mathrm{m} \times 3.45 \mu \mathrm{m}$ and frame size of $2048 \mathrm{px} \times 1536 \mathrm{px}$. The distance from the camera detector array to the object surfaces was set to $20 \mathrm{~cm}$ (camera gain of $0 \mathrm{~dB}$ ) for motorized measurements and $30 \mathrm{~cm}$ (camera gain of $9 \mathrm{~dB}$ ) for handheld measurements, respectively. The distances varied slightly during motorized-rotational and handheld measurements.

Figure 3.1 illustrates the handheld LSCI probe which has a total weight of approximately $750 \mathrm{~g}$ including the connecting cables and optical fiber. The camera objective (FUJINON HF16XA-5M) had a $16 \mathrm{~mm}$ focal length and based on prior measurements on our imaging geometry, the $\mathrm{f}-$ number was set to $F 8$ to obtain the optimum point for (1) the speckle size to meet the Nyquist criterion [18] and (2) the detected light intensity to have the required dynamic range for computation of speckle contrast. With a distance of $20 \mathrm{~cm}$ from the camera sensor array to the object surface, the measured resolution was $26.8 \mathrm{px} / \mathrm{mm}$; hence, the total field-of-view was $7.6 \mathrm{~cm} \times 5.7 \mathrm{~cm}$. A hard coated bandpass interference filter of wavelength $675 \pm 12.5 \mathrm{~nm}$ (Edmund Optics) 
was mounted on the camera objective in order to reduce the background light although the general lighting of the laboratory were turned off during the experiments. To avoid detection of specular reflection from the samples and increase speckle contrast, a linear polarizer optimized for the wavelengths $600-1100 \mathrm{~nm}$ (Thorlabs LPNIRE 100-B) was used in the imaging system with the polarization direction perpendicular to the polarization direction of the laser beam. Note that the output laser beam was linearly polarized and the polarization was partly lost in the single mode optical fibre. To prevent saturating some areas of the camera, for each scattering medium and for each wavefront type, the laser light intensity coupled to the optical fibre was adjusted by displacing the three axis stage on which the optical fiber tip was mounted. The average values of the recorded speckle intensity maps were in the range of $15-25$ out of 255. The relatively low average intensity is due to the use of a single mode optical fiber and its maximum tolerable optical power. This was compensated by adding camera gain while making sure that the speckle contrast stays just below unity when a static sample was imaged and the system was mounted. In previous experiments on the used camera, we confirmed that a gain level of $9 \mathrm{~dB}$ with an average speckle intensity of $15-25$ out of 255 does not have a significant impact on the measured speckle contrast. This is due to the high signal-to-noise ratio of the camera (40.2 dB). Another limiting factor for further increasing average intensity is to prevent saturation at any camera pixel. This average intensity level makes the speckle contrast to fall on the interval $[0.14,1]$ thanks to the rather high quantum efficiency of the used camera (70\%).

\section{Spherical waves}

The optical fiber emitted a diverging beam with a nominal numerical aperture of 0.12 such that the $1 / e^{2}$ beam width at the measurement distance of $20 \mathrm{~cm}$ from the fiber tip to the object surfaces was $3 \mathrm{~cm}$. The processing window sizes (almost in the center of the array) were $150 \mathrm{px} \times 150 \mathrm{px}$ except for handheld measurements on the custom-made phantom which was $120 \mathrm{px} \times 120 \mathrm{px}$.

\section{Scrambled waves}

To create a scrambled beam, a $20^{\circ}$ top hat engineered diffuser (Thorlabs ED1-S20MD) with square scattered shape was mounted at a distance $32.4 \mathrm{~mm}$ from the fiber tip. This created a square of width $7.8 \mathrm{~cm}$ on the object surface for the motorized measurements. The window size to process speckle intensity frames for scrambled waves was set to $150 \mathrm{px} \times 150 \mathrm{px}$. 


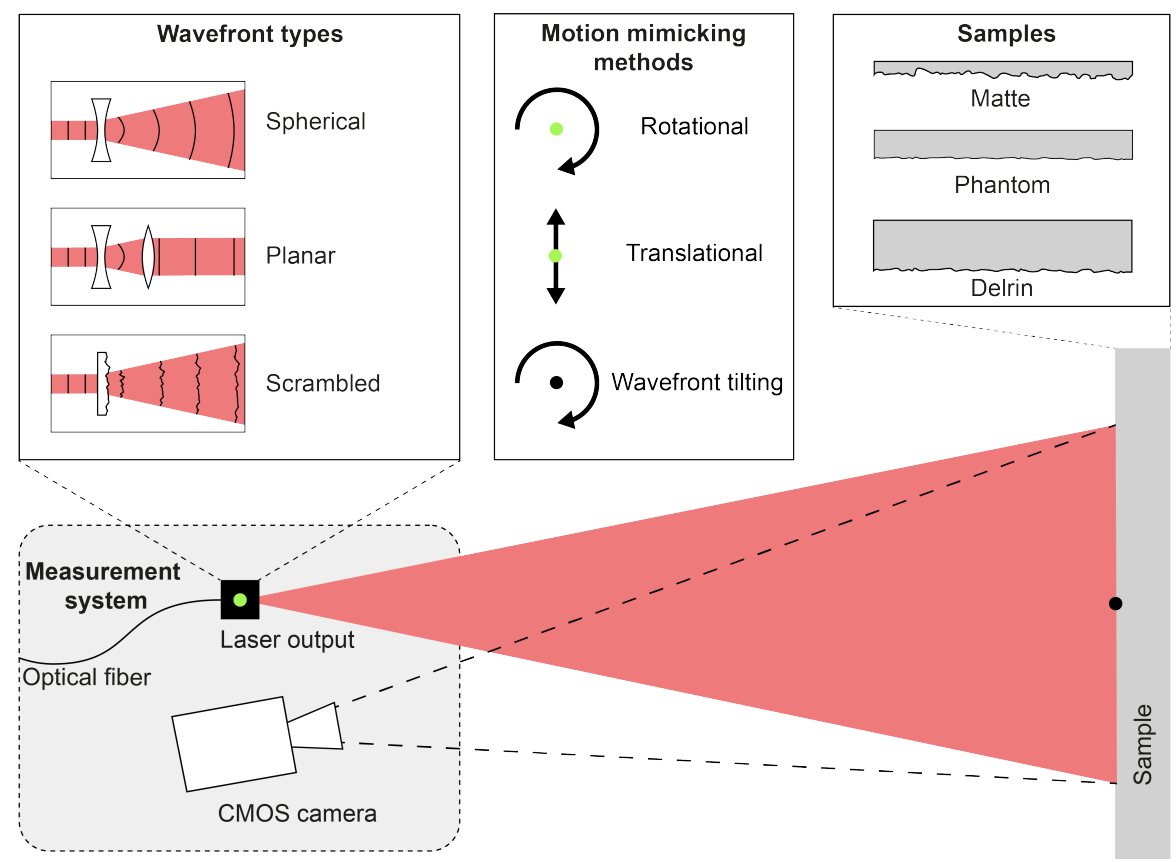

Fig. 3.2: Schematic diagram of the experimental setup for the motorized measurements. For rotational motions, translational motions and wavefront tilting see Visualization 3.1, Visualization 3.2 and Visualization 3.3, respectively. The centers of rotation for system rotation and wavefront tilting are indicated by the green and black dots, respectively.

\section{Planar waves}

A parallel beam of diameter $2.5 \mathrm{~cm}$ with planar wavefronts was created by collimating the laser beam with a pair of plano-convex (Thorlabs LA1608) and bi-convex (Thorlabs LB1945-A-ML) lenses. The distance from the fiber tip to the front side of the bi-convex lens was set to $65.5 \mathrm{~mm}$. For this wavefront type in motorized measurements, the maximum possible window sizes to process speckle intensity maps were $55 \mathrm{px} \times 55 \mathrm{px}$, $70 \mathrm{px} \times 70 \mathrm{px}, 115 \mathrm{px} \times 115 \mathrm{px}$ and $120 \mathrm{px} \times 120 \mathrm{px}$ for rotational movements on Delrin, rotational movements on matte, object rotation on Delrin and object rotation on matte, respectively. It was $150 \mathrm{px} \times 150 \mathrm{px}$ for the translational movements. In the handheld measurements, they were $75 \mathrm{px} \times 75 \mathrm{px}, 65 \mathrm{px} \times 65 \mathrm{px}$ and $65 \mathrm{px} \times 65 \mathrm{px}$ on Delrin, custom-made phantom and matte, respectively.

\section{Aiming beam}

A laser source (RGb Laser Systems Minilas) of wavelength $660 \mathrm{~nm}$ was mounted on the handheld probe to be used as a guide for some of the handheld measurements 
where its beam was projected on a cross target shown in Fig. A.1.

\subsubsection{Controlled movements on motorized stages}

The experimental plan for the motorized measurements is illustrated in Fig. 3.2. Three types of wavefronts, namely spherical, planar and scrambled were included in the experiments. From the sample side, three types of media, namely a metal plate painted with color white matte, Delrin and phantom (to be specified later) were used for the measurements. For the motion mimicking schemes, translational movements parallel to the medium's surface were applied to study the effect of translation of wavefronts; tilt movements were applied to generate tilt of wavefronts; and rotational movements were applied to realize a combination of the first two which is a form of movements occurring during a handheld operation. Note that translation of the system towards the medium's surface is considered negligible due to its small effect on the speckle intensity map. Also, compared to the other types of motion, rotation around the optical axis will be small during a typical handheld operation.

\section{Translation}

The handheld probe (facing to the static objects) was mounted on a linear translational stage (Zaber X-LHM200A-E03) with the travel length of $20 \mathrm{~cm}$, speed range of $0-6.5 \mathrm{~cm} / \mathrm{s}$ and speed resolution of $76 \mathrm{~nm} / \mathrm{s}$. Here the probe moved parallel to the object surface as animated in Visualization 3.2.

\section{Rotation}

A high precision rotation mount (Thorlabs PRM1/M28) with the angular speed range of $0-25 \%$ s and minimum step size of 25 arcsecond was employed to apply rotations. For the rotational movement, the rotation mount was placed between the handheld probe and a fixed vertical bar attached to the optical table such that the green point on the fiber tip became the center of rotation (see Visualization 3.1). For the tilt movement, this rotation mount was attached to the static medium while the handheld probe facing the medium at the distance of $20 \mathrm{~cm}$ (from the camera sensor array to the object surface) was fixated on the optical table such that the black point on the object surface and just in front of the fiber tip became the center of rotation (see Visualization 3.3). The controller for this stage was a brushed DC servo motor unit (Thorlabs KDC101). The speed profiles were applied to the motor unit using the software Kinesis. 


\subsubsection{Static phantoms}

Three scattering media were used in this study. The first medium was a wide plate of thickness $10.9 \mathrm{~mm}$ made of Polyoxymethylene material (Delrin). The second sample, referred to as matte, was a metal plate painted 6 times with spray paint of color white matte (Ecopaint Vintage chalk paint spray $400 \mathrm{ml}$ ). This medium has a very high scattering level; thus, only surface scattering occurs. The third medium was a tissue mimicking scattering phantom of reduced scattering coefficient $2 \mathrm{~mm}^{-1}$ and absorption coefficient $0.01 \mathrm{~mm}^{-1}$. These optical properties for our operating wavelength of $671 \mathrm{~nm}$ were adapted from Lister et al. [19]. Agar powder (Sigma A7921-500G) was used to make the phantom relatively static and reduce the Brownian motions. A stock solution of demi-water at boiling temperature with $2 \%$ agar was prepared. To control the absorption level of the phantom, the Ecoline 700 ink (Talens) was added when the stock solution had cooled down to around $60{ }^{\circ} \mathrm{C}$. Using the spectrophotometer of wavelength $[300-1100 \mathrm{~nm}]$ (Shimadzu UV-2600) the absorption coefficient of Ecoline at $671 \mathrm{~nm}$ was measured as $24.6 \mathrm{~mm}^{-1}$. Then, for making the phantom optically scattering, Intralipid 20\% (Fresenius Kabi Nederland BV) assuming the reduced scattering coefficient of $26 \mathrm{~mm}^{-1}$ [20], was poured to make 7.7 vol\%. The prepared solution was cast in a 3D printed mold of Polylactic Acid (PLA) material with the effective dimension of $140 \times 140 \times 14.1 \mathrm{~mm}^{3}$ and was kept for two hours to reach the room temperature.

\section{In-vivo measurements}

Two healthy male subjects were involved in in-vivo experiments. Translation of the LSCI system as explained in Section 3.2.2 was applied to forearm of the test subjects who were sitting in a comfortable position during the experiments. Subjects were asked to refrain from intensive physical activity at least 30 minutes before start of the experiments.

\subsubsection{Data analysis}

The total acquired speckle intensity frames for translational movements, rotational movements, object rotation and handheld operations were 800, 1000, 1000 and 2400, respectively. The contrast of the region-of-interest (ROI) within each frame was calculated as [21];

$$
C=\frac{\sigma_{I}}{\bar{I}}
$$

where $\sigma_{I}$ and $\bar{I}$ indicate the standard deviation and the mean of pixel values within each frame. In this way, a speckle contrast vector including the baseline and the actual 
measurement was computed per experiment. The equation for speckle contrast drop percentages shown in Tables 3.1,3.2, A.1 and Fig. 3.4(c) is;

$$
\Delta C=100 \frac{C_{0}-C_{1}}{C_{0}},
$$

where $C_{0}$ and $C_{1}$ are speckle contrasts at speed zero and speed of interest, respectively, for the motorized experiments; and the average speckle contrast for baseline and handheld, respectively, for the handheld experiments.

\section{The speckle contrast window size}

In order to achieve an appropriate statistics, we used a rather larger ROI (above $50 \times 50 \mathrm{px}$ ) for the calculation of the speckle contrast than what is normally used (below $10 \times 10 \mathrm{px}$ ) when imaging a tissue subject. We realized that a large ROI may give wrong result if an intensity gradient exists within the ROI. Then, we checked this not to be the case by reanalyzing the data with a window size of $7 \times 7 \mathrm{px}$ at different areas within the ROIs. Also, the variation in the window sizes does not have a significant impact on the observed difference in speckle contrast of the wavefront types at each applied speed. In this regard, we compared graphs of speckle contrast versus translational speed (calculated by both window sizes of $70 \times 70 \mathrm{px}$ and $7 \times 7 \mathrm{px}$ ) for the case of Delrin and matte (See Fig. A.3). While a too small ROI may cause a graph with high random variations, a too large ROI may include artefacts such as reflection. Therefore, an optimized window size is chosen according to the type of movement and illumination to ensure that a uniform speckle pattern is used for the calculation of contrast.

\subsubsection{Handheld measurement protocol}

Six and 9 healthy volunteers with normal hand tremor participated in the experiments with various beams and with the aiming beam, respectively. Subjects were asked to avoid over-concentration during the handheld measurements since the purpose was to realize movements of the LSCI system during normal handheld operations. During the first $10 \mathrm{~s}$ of the image acquisition, the handheld LSCI system was mounted on the rest position to make a baseline measurement. Then, it was lifted slowly and kept still by subjects standing normal in front of the setup and with arm bent at elbow at $90^{\circ}$ for a $40 \mathrm{~s}$ handheld measurement (Fig. 3.1). The distance from the camera sensor array to the imaged object was almost $30 \mathrm{~cm}$. The measurements were carried out on 3 scattering media ( 3 series) each with 3 beam types to obtain the average speckle contrast. In each series, 6 test subjects participated and to prevent the issue of fatigue, a gap of at least 45 minutes between measurements was considered for each test subject. The same experiment protocol was followed for the experiments of aiming beam where 9 test subjects were included in the study. 


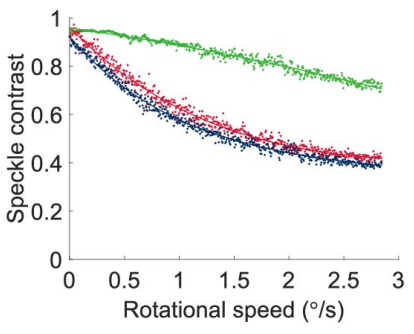

(a)

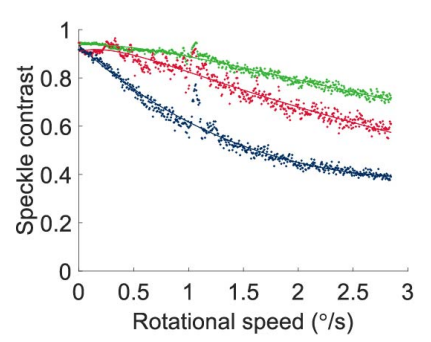

(d)

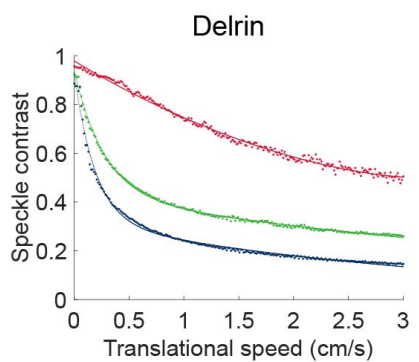

(b)

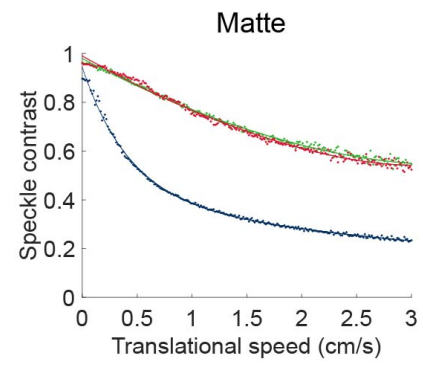

(e)

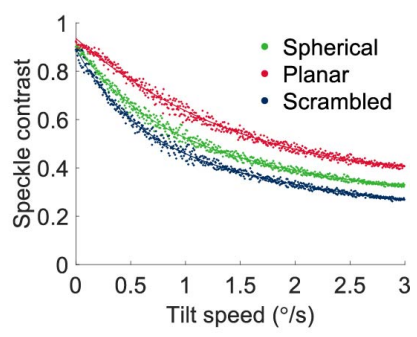

(c)

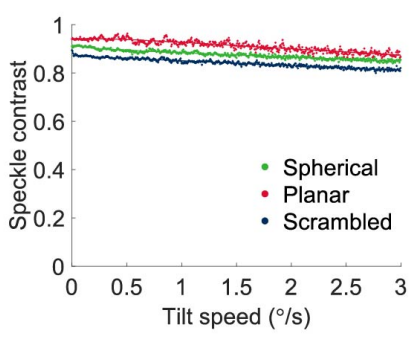

(f)

Fig. 3.3: Motorized movements of the LSCI system (a-c) on a Delrin plate and (d-f) on a matte surface for spherical, planar and scrambled wavefronts. Speckle contrast vs $(a, d)$ rotational and $(b, e)$ translational speeds. (c,f) Speckle contrast versus applied tilt speed realized by object rotation. For spherical, planar and scrambled wavefronts see Visualization 3.4, Visualization 3.5 and Visualization 3.6, respectively, where translational motions are applied on a Delrin plate. For rotational motions and object rotation see Visualization 3.7 and Visualization 3.8, respectively, where a Delrin plate and spherical wavefronts are employed. Solid curves: second order exponential fit functions.

\subsection{Results}

\subsubsection{Motorized measurements}

First, the LSCI system was rotated from an angle normal to the object surface to the angle of $22.5^{\circ}$ with an acceleration of $0.2^{\circ} / \mathrm{s}^{2}$ in 15 seconds time. The same angular speed was applied for spherical, planar and scrambled beam types. The speckle contrast vs. the applied rotational speed is depicted in Fig. 3.3(a,d), for Delrin and matte, respectively. The drop in speckle contrast is smallest for the spherical wavefront and largest for the scrambled wavefront, and for these beams the contrast drops are quite similar for both media. The behavior for the planar waves is in between those of the other beam types, with a larger sensitivity for Delrin than for matte.

Second, translational displacement of the system over $8.7 \mathrm{~cm}$ in 5.8 seconds time with an acceleration of $5.2 \mathrm{~mm} / \mathrm{s}^{2}$ was applied. See Fig. 3.3(b,e), for the Delrin and matte objects, respectively. Visual comparisons of speckle intensity maps and the 


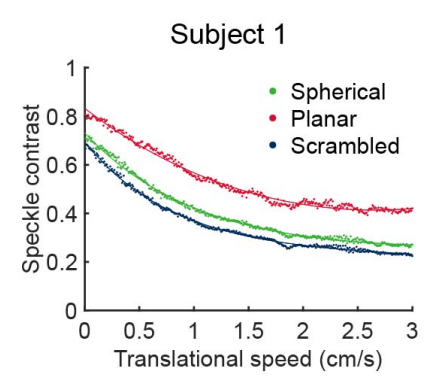

(a)

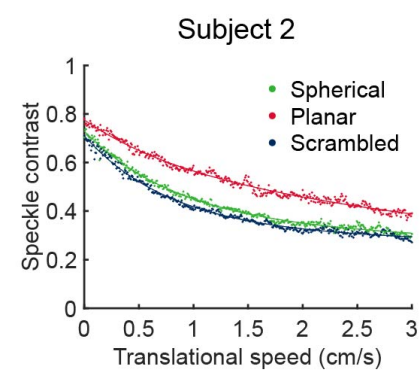

(b)

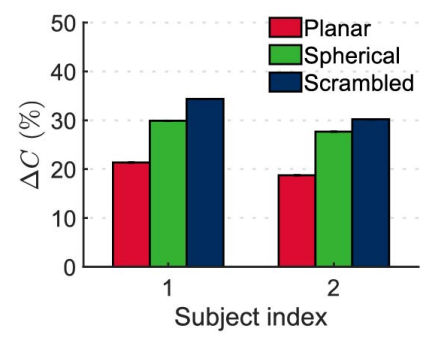

(c)

Fig. 3.4: Motorized translation of the LSCI system on forearm of two healthy subjects. (a-b) Speckle contrast versus translational speed for subjects 1 and 2, respectively. Solid curves: second order exponential fit functions. (c) Speckle contrast drop percentages (introduced in Eq. (3.2)) from the maximum values at zero speed to the translational speed of $0.6 \mathrm{~cm} / \mathrm{s}$. Based on our previous study [22], the translational speed of $0.6 \mathrm{~cm} / \mathrm{s}$ is the averaged value of a number of handheld measurements employing an EM-tracking system. Data of (c) are extracted from (a-b).

corresponding speckle contrast vs applied translational speed on Delrin for spherical, planar and scrambled wavefronts are provided in Visualization 3.4, Visualization 3.5 and Visualization 3.6, respectively. Here the beam with planar wavefronts has the lowest sensitivity to motion and the scrambled beam the highest, and for these beams the contrast drops are quite similar for both media. The spherical wavefronts give a result in between for Delrin, while giving the same contrast vs speed relation as the planar wavefronts for matte.

Third, a tilt of spherical, planar and scrambled wavefronts was realized by rotating the samples with the same angular speed profile as the first series, that is, the acceleration of $0.2 \% \mathrm{~s}^{2}$ in 15 seconds time. Here the samples started from the angle of $25^{\circ}$ with respect to the optical fibre axis and traveled an arc of $22.5^{\circ}$ towards optical fibre axis. Speckle contrast values vs tilt speed for Delrin and matte are shown in Figs. 3.3(c,f), respectively. For matte there is only a small drop of speckle contrast for all beam types. For Delrin the contrast drops are much larger. For both media, the difference between the three beam types is much smaller for tilt and for translation or rotation. A visual comparison of speckle intensity maps between the three movement types (i.e. rotation, translation and tilt) with the spherical beams on Delrin can be found in Visualization 3.7, Visualization 3.4 and Visualization 3.8, respectively. In general, the scrambled beam shows the largest sensitivity to object motion.

\section{In-vivo validation of translation}

LSCI measurements on the forearm of two subjects have been performed as introduced in Section 3.2.3. Figure 3.4(a-b) show the results of speckle contrast versus transla- 


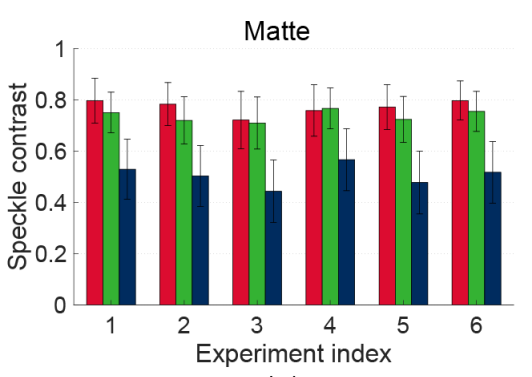

(a)

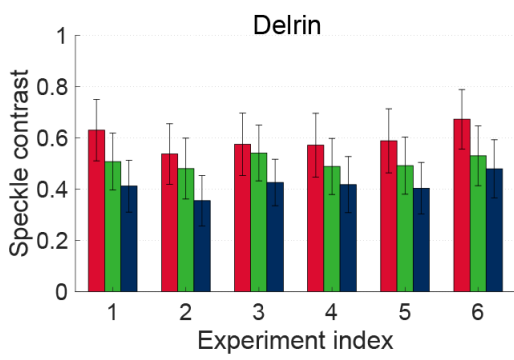

(c)

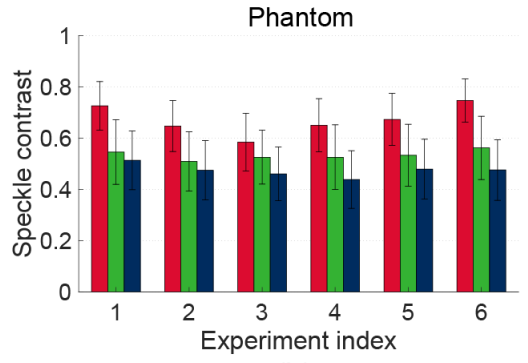

(b)

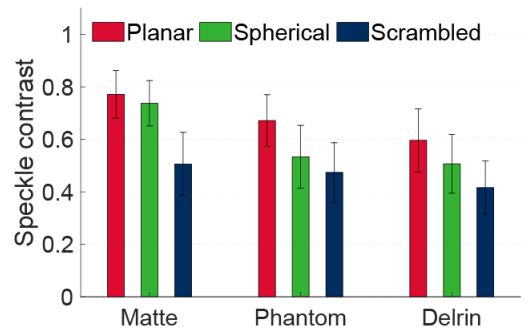

(d)

Fig. 3.5: Speckle contrast of handheld measurements on (a) matte, (b) agar-Intralipid phantom and (c) Delrin and for planar, spherical and scrambled wavefronts. (d) Speckle contrast (mean \pm standard deviation) for various scattering media and wavefronts.

tional speed for the three types of wavefronts. The speckle contrast values at zero speed are lower than the case of static objects shown in Fig. 3.3 which is due to the cutaneous microcirculatory blood flow. The difference in the speckle contrast values at zero speed between the various beam types, especially for subject 1, is larger than in-vitro (Delrin or matte). This can be due to the change in blood flow of the imaged regions. Therefore, in order to perform a fair comparison between the wavefront types, speckle contrast drop percentages introduced in Eq. (3.2) with $C_{0}$ and $C_{1}$ being the speckle contrast values at zero speed and translational speed of $0.6 \mathrm{~cm} / \mathrm{s}$, respectively, have been calculated. As shown in Fig. 3.4(c), for subject 1, the least speckle contrast drop percentage has been achieved with planar wave (21.3\%), compared to spherical wave $(29.9 \%)$ and scrambled wave $(34.4 \%)$. Similarly, for subject 2 , the least speckle contrast drop percentage has been achieved with planar wave (18.7\%), compared to spherical wave $(27.7 \%)$ and scrambled wave $(30.2 \%)$. This suggests that plane waves cause the least movement artefacts when the LSCI system is purely translated. 


\subsubsection{Handheld measurements}

The results of the 3 series of the handheld measurements, namely, on matte, on agarIntralipid phantom, and on Delrin plate are depicted in Figs. 3.5(a-c), respectively, where each experiment index per graph refers to an identical test subject. Therefore, the data of 3 groups of 6 test subjects are shown. Note that the members of mutual groups are not necessarily the same. To have an outlook on the three series, the data of 6 experiment indices within each series are averaged (Fig. 3.5(d)).

The last step of the study was devoted to the potential difference an aiming beam might make in terms of speckle contrast during handheld measurements. Therefore, subjects operated handheld measurements on the Delrin plate, with spherical beam and having an aiming beam mounted on the handheld LSCI system. The comparison was made with the data of that of Delrin, with spherical beam and without an aiming beam. The averaged speckle contrast for 9 test subjects is shown in Fig. A.2 where each experiment index refers to an identical test subject and the last column pair shows the average value of the experiment indices.

\subsubsection{Hardware and data analysis considerations}

\section{Use of a linear polarizer}

As mentioned in Section 2.1, a linear polarizer is mounted on the imaging system. The direction of the polarizer is perpendicular to the direction of the polarization of the light source in order to minimize the artefact caused by specular reflection (see Fig. A.4). It is worth noting that the use of a polarizer helps to detect nearly fully developed speckle patterns in case of no movements at the expense of the intensity loss. The underlying reason is filtering the portion of the backscattered light with various polarization directions except that of parallel with the direction of the linear polarizer. Therefore, a higher dynamic range with respect to the speckle contrast is achieved.

\section{The Gaussian beam illumination}

Apart from the engineered diffusers being compact, they provide a uniform illumination on the target tissue. Since the speckle contrast introduced in Eq. (3.1) is independent of the intensity level, a uniform illumination is not a requirement for obtaining a valid speckle contrast map from a wide tissue area. Note that in order to minimize the possibility that the speckle contrast is influenced by intensity gradient, (1) the mean intensity should not be so high that parts of the camera sensor is saturated; (2) the mean intensity should not be so low (below $5 \%$ of the maximum observable value) that the camera noise level becomes comparable with the detected intensity level. The laser light after expansion on a tissue surface is of a Gaussian nature. 
The larger the distance from the center of the beam, the lower the observed intensity. Therefore, as long as the mean intensity falls within the valid range, illuminating a target tissue with spherical wave illumination would not affect the relative speckle contrast at different locations of the illuminated tissue (see Fig. A.5).

\subsection{Discussion}

In this work, we focused on illumination schemes for handheld LSCI and their influence on the movement artefacts studied by measuring speckle contrast during motorized and human handheld measurements on the extreme cases of high scattering (matte) and low scattering (Delrin plate) and on a tissue mimicking phantom. To exclude any other source of movements than the LSCI system, experiments were carried out on static scattering media. The first phase of the study consisted of applying three motion mimicking methods (i.e. translational, rotational and object rotation) to the system. The results are shown in Fig. 3.3. It appears that, for each type of applied motion, the relation between the speckle contrast and the amount of applied motion depends on (1) the type of the illuminations and (2) the scattering level of the media. In general, the contrast's sensitivity to motion increases with decreasing scattering level, and scrambled wavefronts give a higher motion sensitivity than spherical and planar wavefronts. For media with tissue-like scattering properties such as Delrin (Fig. 3.3(a-c)), planar waves give a lower sensitivity than spherical and scrambled waves.

To address these observations, we take a closer look at the system geometry and use the optical Doppler effect. We regard the tip of the optical fiber as a point source emitting spherical waves. Figure 3.6(a) shows a snapshot of wavefronts at some distance from the source and the corresponding illuminating wave vectors $\vec{k}_{i}(x, y, z)$, where $k=2 \pi / \lambda$ is the wave number. Here the distance and the wavelength are considered as $z=20 \mathrm{~cm}$ and $\lambda=671 \mathrm{~nm}$. For a circle in millimeter scale radius, e.g. $a=1 \mathrm{~mm}$ in the $x y$ plane centered about the $z$ axis, the wavefronts may look curved with the Fresnel number of $F_{N}=a^{2} /(\lambda z)=7.4$ which is greater than unity. In other words, within a typical distance over which light diffuses from the point of injection to the point of detection, the wavefront is significantly curved. This contributes to a range of incoming wave vectors when the medium has a low scattering level such as Delrin. In Fig. 3.6(b), a collimating system is used to create plane waves having a constant wave vector toward the scattering medium. In Fig. 3.6(c), an engineered diffuser is used to create scrambled waves. In this case, each illuminated point of the diffuser is a point source of light with random phase. As a consequence, each location on the surface of the scattering medium is illuminated by light with a range of wave vectors with a small angular variation.

The light entering the scattering medium travels random pathways and a portion of 


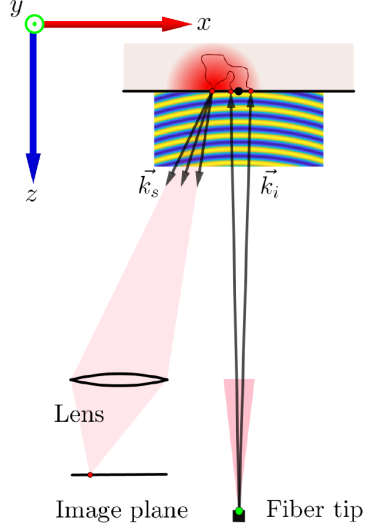

(a)

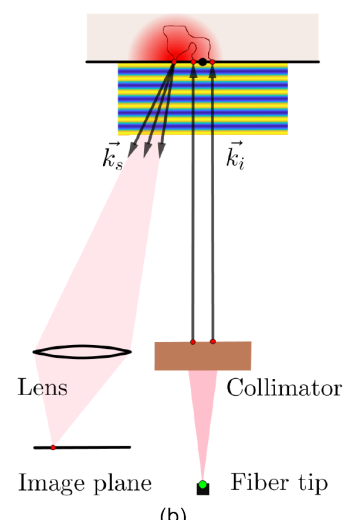

(b)

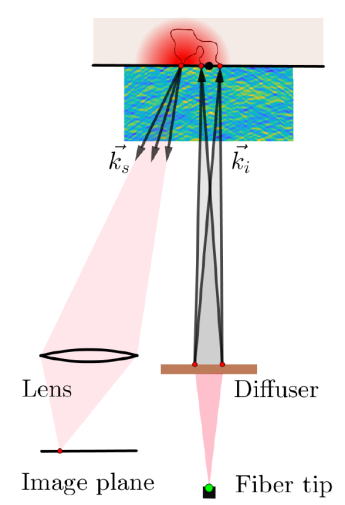

(c)

Fig. 3.6: Schematic diagram of illumination and imaging systems in a reflection geometry where the scattering medium is illuminated with (a) spherical, (b) planar and (c) scrambled waves. $\vec{k}_{i}$ : incoming wave vectors (normals to the wavefronts) toward the scattering medium; $\overrightarrow{k_{s}}$ : outgoing wave vectors from a scattering spot collected by the imaging lens. The half circle with gradient red color represents the fluence distribution of light eventually being imaged on a certain point in the imaging plane. The solid lines within the fluence distribution show random pathways. Green circles on the fiber tips: center point for rotation of the LSCI system in the experiments of rotational displacements; Black circles: center point for rotation of the medium in the experiments of wavefront tilting. Scales are exaggerated for the sake of demonstration.

it called the diffused light comes back to the surface. The fluence distributions shown in Fig. 3.6 symbolically represent the fluence of light returning to the tissue surface in a point conjugated with a certain position on the imaging plane within a cone defined by the imaging system. The range of wave vectors $\vec{k}_{s}(x, y, z)$ of detected light is the same for the three illumination schemes since the imaging geometry remained unchanged. The time scale of intensity fluctuations in the image plane determines the blurring of the time integrated speckle pattern and therefore its contrast. It is the variation of the optical Doppler shifts that determines the time scale of speckle intensity fluctuations (therefore, the blurring level of the time integrated speckle patterns) rather than the absolute values of the optical Doppler shifts. The reason is that intensity fluctuations are the result of interference between light fractions with a different Doppler shift: if all light had the same Doppler shift, there would be no intensity fluctuations.

First we discuss the case of linear translation. If the optical system moves with velocity $\vec{v}$ along the $x$ axis relative to the measured medium, i.e. $\vec{v}=\left(v_{x}, 0,0\right)$, the Doppler shift of light obeys [23]

$$
\Delta \omega=\left(\vec{k}_{s}-\vec{k}_{i}\right) \cdot \vec{v}
$$

independent of the details of the light paths inside the medium. A variation of wave 
vectors $\vec{k}_{s}$ and $\vec{k}_{i}$ will cause a range of Doppler shifts leading to intensity fluctuations. The spread of detected wavevectors $\vec{k}_{s}$ depends only on the lens aperture which is kept unchanged during this study. Assuming a lens aperture opening or closing of $1 \mathrm{~mm}$, the maximum two-sided Doppler shift of light per each $\mathrm{mm} / \mathrm{s}$ of translational speed based on Eq. (3.3) is $7.45 \mathrm{~Hz}$, for the wavelength of $671 \mathrm{~nm}$ and the distance from the object's surface to the lens aperture of $20 \mathrm{~cm}$. Due to the high scattering properties of matte, only one incoming wave vector $\vec{k}_{i}$ contributes to the creation of Doppler shift for the planar and spherical waves. This results in the same response to the applied translational speed for the planar and spherical waves (see Fig. 3.3(e)). Moreover, for the plane waves, there is only one incoming wave vector $\vec{k}_{i}$ that makes the results for Delrin and matte the same, as shown in Fig. 3.3(b,e). For the spherical waves, the Doppler shift range will be greater than the plane waves since the scattering spot contains a range of incoming wave vectors (Fig. 3.3(b)). As an example, consider a scattering spot of radius $a=1 \mathrm{~mm}$. The maximum two-sided Doppler shift of light based on Eq. (3.3) can be calculated (in Hertz) as $\Delta v=2 a v /(\lambda z)=14.9 \mathrm{~Hz}$ per each $\mathrm{mm} / \mathrm{s}$ of translational speed for the spherical waves at $\lambda=671 \mathrm{~nm}$ and $z=20 \mathrm{~cm}$. This maximum frequency exists in the power spectrum of intensity fluctuations observed at the point on the camera sensor conjugated with the points of radius $a=1 \mathrm{~mm}$ located on the medium's surface. As a consequence, for an exposure time of $10 \mathrm{~ms}$, the calculated Doppler shift can be expected to cause a non-negligible speckle contrast drop. Since a range of incoming wave vectors contribute to each location on the scattering spot caused by scrambled waves, the resultant Doppler shift range will be more than that of planar and spherical waves. For the same reason as that for spherical waves, the Doppler shifts will be less in the case of higher scattering medium for the scrambled waves. Finally, the results for the spherical beam is much more medium dependent than for the planar waves and the scrambled waves, as can be seen in Fig. 3.3(b,e). To examine the reproducibility of the data, this experiment has been carried out again (see Fig. A.3(a-b) where the results are in agreement with Fig. $3.3(\mathrm{~b}, \mathrm{e})$. Note that the range of incoming wave vectors $\vec{k}_{i}$ dictates the phase structure of the incident light on the sample. This phase structure is constant for plane waves. It gradually changes for the spherical waves. And it is randomly distributed for the case of scrambled waves.

Rotation of the illumination and imaging systems along the fiber tip (the green point located on the fiber tip shown in Fig. 3.6) with a certain angular velocity will also cause Doppler shifts. Equation (3.3) cannot be used with its current form since for rotation the details of the light trajectories will matter. Since the fiber tip is also origin for propagation of spherical waves, the incoming wave vectors tend to match the curve of rotation and since the outgoing wave vectors also rotate in the same direction, the dependence of the Doppler shift on angular speed is minimum for the case of spherical waves. This would not be the case for planar waves since the incoming 
Table 3.1: Speckle contrast drop percentages from the maximum values at zero speed to rotational speed of $1.1 \%$ s, translational speed of $0.6 \mathrm{~cm} / \mathrm{s}$ and tilt speed of $1.1 \% \mathrm{~s}$ measured on Delrin and matte. Based on our previous study [22], these are averaged values of a number of handheld measurements employing an EM-tracking system. Data are extracted from Fig. 3.3.

\begin{tabular}{l|ccc|ccc} 
& \multicolumn{3}{c}{ Delrin } & \multicolumn{3}{c}{ Matte } \\
& Spherical & Planar & Scrambled & Spherical & Planar & Scrambled \\
\hline \hline Rotational & 7.4 & 33.7 & 38.2 & 5.3 & 13.7 & 35.9 \\
Translational & 51.1 & 12.5 & 65.2 & 10.4 & 10.4 & 45.6 \\
Tilt & 42.2 & 33.7 & 49.4 & 3.3 & 2.1 & 5.6
\end{tabular}

wave vectors exist in one direction and rotation of the system causes the change in the relative angles of incoming and outgoing wave vectors (Fig. 3.3(a)). What is more, for the plane waves, these relative angles also depend on the scattering level of the medium. As the scattering spot becomes smaller with higher levels of scattering, the caused Doppler shift range decreases (Fig. 3.3(d)). However, this is not the case for the spherical and the scrambled waves as they result in the same behavior in the case of matte and Delrin.

Now consider wavefront tilt, generated by rotation of the object along a point on its surface just in front of the fiber tip on the optical axis (the black points shown in Fig. 3.6). In case of very high scattering and therefore a very small scattering spot, the incoming and outgoing wave vectors come from the same location on the medium's surface. If this location is close enough to the point of rotation, the effective velocity contributing to the Doppler shift remains zero. In practice, for wavefront tilting we observed relatively low speckle contrast drop for all three illumination schemes for measurements on matte as depicted in Fig. 3.3(f). The slight decay of all three curves is because the ROI includes the area around the center of rotation. As this ROI increases, we expect more contrast drop. For Delrin, a less scattering medium, the scattering spot will be wider and this results in a larger differences between the incoming and outgoing wave vectors. Furthermore, in Delrin, tilting causes a space dependent velocity component of the tissue towards or away from the LSCI system, causing a rather larger contrast drop for all three beam types (see Fig. 3.3(c)). To sum up, for all beam types, there is a larger sensitivity to motion for tilt speed for Delrin, and an almost zero sensitivity for matte for tilt speed. A summary of the speckle contrast drops for the motorized measurements depicted in Table 3.1 suggests that the least speckle contrast drop is achieved by using (1) spherical waves while applying rotations; (2) planar waves while applying translations or tilt. This means that the choice of appropriate illumination potentially controls system sensitivity to the applied 
Table 3.2: Speckle contrast drop percentages from the averaged values at baselines to the averaged values of the associated handheld measurements shown in Fig. 3.5. Avg.: Averaged.

\begin{tabular}{cccccccc|c} 
& & \multicolumn{7}{c}{ Experiment index } \\
& & 1 & 2 & 3 & 4 & 5 & 6 & Avg. \\
\hline \multirow{3}{*}{ Matte } & Planar & 11.4 & 12.2 & 17 & 16.9 & 14.2 & 8.9 & 13.4 \\
& Spherical & 17.1 & 21.4 & 21.6 & 15.9 & 20.6 & 16.9 & 18.9 \\
& Scrambled & 42.3 & 45.8 & 51.6 & 37.8 & 47.4 & 43.7 & 44.7 \\
\hline \multirow{3}{*}{ Phantom } & Planar & 21.3 & 30.2 & 36.1 & 27.6 & 25.7 & 18.8 & 26.6 \\
\cline { 2 - 8 } & Spherical & 39 & 44.1 & 41.6 & 41.5 & 41.3 & 38.5 & 41 \\
& Scrambled & 43.4 & 47.5 & 48.5 & 51.4 & 46.7 & 47.9 & 47.6 \\
\hline \multirow{3}{*}{ Delrin } & Planar & 32.2 & 41.3 & 37.5 & 36.4 & 36.2 & 25 & 34.8 \\
& Spherical & 43.5 & 45.6 & 39 & 44.7 & 44.7 & 40.4 & 43 \\
& Scrambled & 54.2 & 60.4 & 52.4 & 53.7 & 54.9 & 45.7 & 53.6
\end{tabular}

movements.

In the handheld measurements, a combination of all of the aforementioned movements takes place. Also, the magnitude of each motion type varies per person as in a previous study [22], we measured an averaged on-surface beam speed and tilt speed of $0.9 \mathrm{~cm} / \mathrm{s}$ and $1.1 \%$, respectively, for ten healthy operators using an electromagnetic (EM) tracking system. Table 3.2 summarizes the speckle contrast drop percentages from the baseline to the averaged values shown in Fig. 3.5. As the medium becomes less scattering, the speckle contrast drop percentages increase (for all beam types). On average, the scrambled waves created by the engineered diffuser cause the lowest speckle contrast (or the greatest speckle contrast drop) in either illumination schemes and in either scattering samples, while the planar wavefronts result in the highest averaged speckle contrast (or the lowest speckle contrast drop). The speckle contrast drop percentages from the averaged baseline to the values of the handheld measurements without and with an aiming beam shown in Fig. A.2 are summarized in Table A.1. The difference in the speckle contrast drop percentages tend to vary per test subject. For some, using an aiming beam causes less drop percentages while for others the other way round. On average, the results stay close to each other: $43.3 \%$ with and $42.6 \%$ without using an aiming beam. Therefore, one can conclude that for this group of test subjects while employing spherical illumination, use of an aiming beam does not decrease the movement artefacts.

Nowadays, most of the laser speckle perfusion imaging systems use engineered diffusers because it is an easy method for creating uniform illumination. Here, we have shown that using engineered diffusers is the worst option in terms of movement artefacts, with planar wavefronts being the best option. However, creating a planar 
beam of large size requires bulky optics. Furthermore, planar and spherical waves make a non-uniform Gaussian-shape light intensity which might affect measurement of speckle contrast at ROIs a couple of centimeters away from the beam center due to a lower average intensity. Results of this study can be used to devise solutions for suppressing movement artefacts in future handheld LSCI systems.

\section{Acknowledgments}

We are grateful to Sjoukje M. Schoustra for designing Fig. 3.2. We thank all the persons who helped us with the handheld operations. This study was supported by the Open Technology program of the Netherlands Organization for Scientific Research (NWO), Domain Applied and Engineering Sciences, under grant number 14538.

\section{Data availability}

Data underlying the results presented in this paper are available in Dataset 1 [17].

\section{References}

[1] M. Draijer, E. Hondebrink, T. van Leeuwen, and W. Steenbergen, "Review of laser speckle contrast techniques for visualizing tissue perfusion", Lasers in Medical Science 24, 639-651 (2008).

[2] D. A. Boas and A. K. Dunn, "Laser speckle contrast imaging in biomedical optics", Journal of Biomedical Optics 15, 011109 (2010).

[3] D. D. Duncan and S. J. Kirkpatrick, "Can laser speckle flowmetry be made a quantitative tool?”, JOSA A 25, 2088-2094 (2008).

[4] J. D. Briers, "Laser speckle contrast imaging for measuring blood flow.", Optica Applicata 37 (2007).

[5] J. Senarathna, A. Rege, N. Li, and N. V. Thakor, "Laser speckle contrast imaging: Theory, instrumentation and applications", IEEE Reviews in Biomedical Engineering 6, 99-110 (2013).

[6] W. Heeman, W. Steenbergen, G. M. van Dam, and E. C. Boerma, "Clinical applications of laser speckle contrast imaging: a review”, Journal of Biomedical Optics 24, 1 (2019).

[7] O. A. Mennes, J. J. van Netten, J. G. van Baal, and W. Steenbergen, “Assessment of microcirculation in the diabetic foot with laser speckle contrast imaging", Physiological Measurement 40, 065002 (2019). 
[8] A. Rege, S. I. Cunningham, Y. Liu, K. Raje, S. Kalarn, M. J. Brooke, L. Schocket, S. Scott, A. Shafi, L. Toledo, and O. J. Saeedi, "Noninvasive assessment of retinal blood flow using a novel handheld laser speckle contrast imager", Translational Vision Science \& Technology 7, 7 (2018).

[9] J. Zötterman, R. Mirdell, S. Horsten, S. Farnebo, and E. Tesselaar, "Methodological concerns with laser speckle contrast imaging in clinical evaluation of microcirculation", PLOS ONE 12, e0174703 (2017).

[10] G. Mahé, P. Rousseau, S. Durand, S. Bricq, G. Leftheriotis, and P. Abraham, "Laser speckle contrast imaging accurately measures blood flow over moving skin surfaces", Microvascular Research 81, 183-188 (2011).

[11] G. Mahe, P. Abraham, A. L. Faucheur, A. Bruneau, A. Humeau-Heurtier, and S. Durand, "Cutaneous microvascular functional assessment during exercise: a novel approach using laser speckle contrast imaging", Pflugers Archiv - European Journal of Physiology 465, 451-458 (2013).

[12] L. Omarjee, I. Signolet, A. Humeau-Heutier, L. Martin, D. Henrion, and P. Abraham, "Optimisation of movement detection and artifact removal during laser speckle contrast imaging”, Microvascular Research 97, 75-80 (2015).

[13] B. Lertsakdadet, B. Y. Yang, C. E. Dunn, A. Ponticorvo, C. Crouzet, N. Bernal, A. J. Durkin, and B. Choi, "Correcting for motion artifact in handheld laser speckle images", Journal of Biomedical Optics 23, 1 (2018).

[14] B. Lertsakdadet, C. Dunn, A. Bahani, C. Crouzet, and B. Choi, "Handheld motion stabilized laser speckle imaging", Biomedical Optics Express 10, 5149 (2019).

[15] L. M. Richards, E. L. Towle, D. J. Fox, and A. K. Dunn, "Intraoperative laser speckle contrast imaging with retrospective motion correction for quantitative assessment of cerebral blood flow", Neurophotonics 1, 015006 (2014).

[16] D. D. Postnov, X. Cheng, S. E. Erdener, and D. A. Boas, "Choosing a laser for laser speckle contrast imaging", Scientific Reports 9 (2019).

[17] A. Chizari, T. Knop, W. Tsong, S. Schwieters, and W. Steenbergen, "Dataset for influence of wavefront types on movement artefacts in handheld laser speckle contrast perfusion imaging", Figshare [retrieved 1 June 2021] (2021).

[18] S. J. Kirkpatrick, D. D. Duncan, and E. M. Wells-Gray, "Detrimental effects of specklepixel size matching in laser speckle contrast imaging", Optics Letters 33, 2886 (2008).

[19] T. Lister, P. A. Wright, and P. H. Chappell, "Optical properties of human skin”, Journal of Biomedical Optics 17, 0909011 (2012).

[20] R. Michels, F. Foschum, and A. Kienle, "Optical properties of fat emulsions", Optics Express 16, 5907 (2008). 
[21] J. W. Goodman, Speckle phenomena in optics: theory and applications (Roberts and Company Publishers) (2007).

[22] A. Chizari, T. Knop, B. Sirmacek, F. van der Heijden, and W. Steenbergen, "Exploration of movement artefacts in handheld laser speckle contrast perfusion imaging", Biomedical Optics Express 11, 2352-2365 (2020).

[23] J. Abbiss, T. Chubb, and E. Pike, "Laser doppler anemometry", Optics \& Laser Technology 6, 249-261 (1974). 
Appendices 



\section{Supplementary information}

\section{Aiming beam}

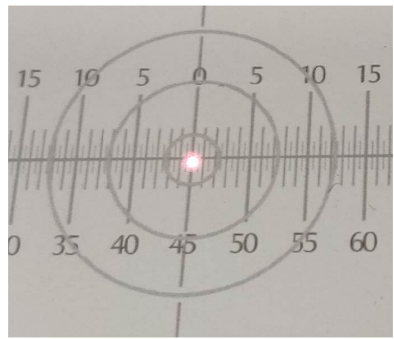

Fig. A.1: Beam target mounted on the static objects for the measurements with the aiming beam. 


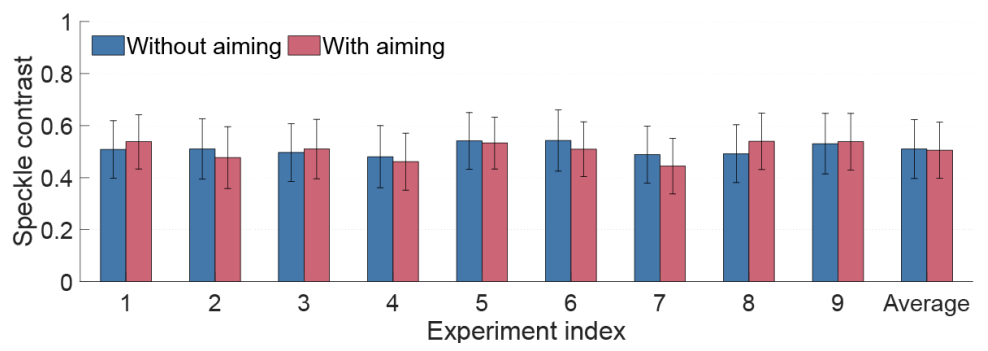

Fig. A.2: Speckle contrast for handheld measurements without and with an aiming beam with spherical beam on a Delrin plate. Data are mean \pm standard deviation.

Table A.1: Speckle contrast drop percentages from the averaged values at baselines to the averaged values of the associated handheld measurements with and without aiming beam shown in Fig. A.2.

\begin{tabular}{cccccc} 
Experiment index & 1 & 2 & 3 & 4 & 5 \\
\hline Without aiming & 43.5 & 42.4 & 43.9 & 45.6 & 39 \\
\cline { 2 - 6 } With aiming & 39.9 & 46.5 & 43.4 & 48.1 & 40.1 \\
& & & & & \\
Experiment index & 6 & 7 & 8 & 9 & Average \\
\hline Without aiming & 39.8 & 44.7 & 44.7 & 40.4 & 42.6 \\
\cline { 2 - 6 } With aiming & 42.6 & 50 & 39.6 & 39.9 & 43.3
\end{tabular}




\section{Replication of motorized translational experiments}

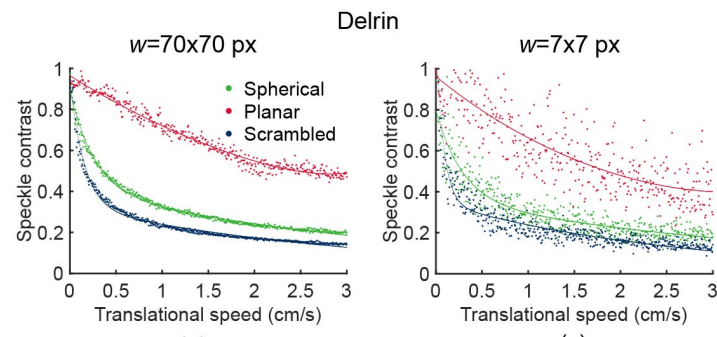

(a)

(c)

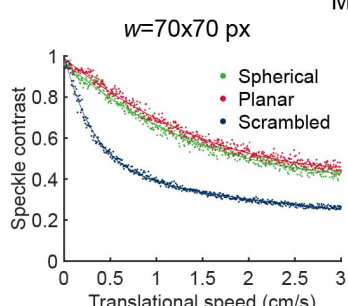

(b)

Matte

$w=7 \times 7 \mathrm{px}$

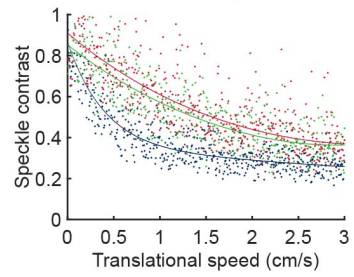

(d)

Fig. A.3: Reproducing motorized experiments for the three wavefront types. Speckle contrast versus translational speed for (a) Delrin and (b) matte with $w=70 \times 70$ px. $w$ : window size for calculation of speckle contrast. Data of (c-d) are associated with (a-b), respectively, where $w=7 \times 7$ px. Solid curves: second order exponential fit functions. 


\section{Use of a linear polarizer}

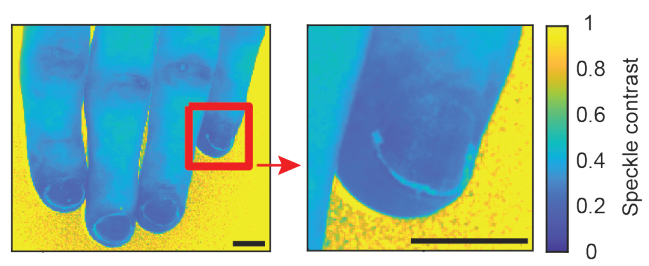

(a)

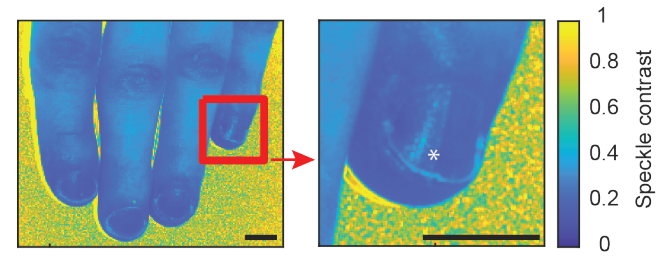

(b)

Fig. A.4: Speckle contrast maps of a backhand (window size of $7 \times 7 \mathrm{px}$ ) using the spherical wave illumination. (a) The speckle contrast map with mounting a linear polarizer on the imaging system with a direction perpendicular to the polarization of the light source. (b) The speckle contrast map without mounting any polarizer. Scale bars, $10 \mathrm{~mm}$. The white asterisk in (b) indicates the region at which the specular reflection artefact in apparent.

\section{Backhand imaging with spherical and scrambled illumina- tions}

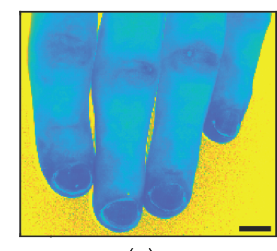

(a)

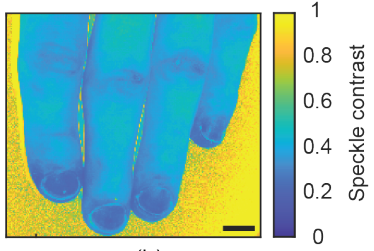

(b)

Fig. A.5: Speckle contrast maps of a backhand (window size of $7 \times 7 \mathrm{px}$ ) using (a) spherical wave and (b) scrambled wave illuminations. scale bars, $10 \mathrm{~mm}$. 


\section{4}

\section{Prediction of movement artefacts caused by translation in handheld laser speckle contrast perfusion imaging}

Movement artefacts distort handheld measurements of laser speckle contrast imaging (LSCI). Enabling a robust LSCI in handheld use brings convenience for both patients and clinical staff. However, there is a lack of a comprehensive model that can predict and potentially compensate the amount of movement artefacts occurring during a handheld LSCI measurement. Here, we propose an analytical-numerical model based on the optical Doppler effect for prediction of movement artefacts caused by translation in a handheld LSCI when static scattering objects are considered. The model incorporates the type of illumination as well as the imaging geometry by taking into account the spread of wavevectors for illumination and detection. We validate the model on numerically simulated speckle patterns. Results of the speckle simulation are in agreement with predictions of the numerical model for both semi-circular and Gaussian forms of the density functions of the incoming and outgoing wavevectors.

${ }^{*}$ Chizari, A. ${ }^{+}$, Tsong W. ${ }^{+}$, Knop, T. and Steenbergen, W., Prediction of movement artefacts caused by translation in handheld laser speckle contrast perfusion imaging. (to be submitted). ${ }^{+}$These authors contributed equally. 


\subsection{Introduction}

Four decades ago, a technique was developed for measurement of spatiotemporal blood flow variations in the microcirculation, by the analysis of laser speckle [1]. Since then, this technique, known as laser speckle contrast imaging (LSCI) [2-4], is advanced in different aspects such as enhancement of spatial resolution [5], processing time [6] and quantitative prediction of blood flow [7-9]. When coherent light encounters an optically rough surface or propagates through a scattering medium such as tissue, an interference pattern is observed on an image screen called speckle. Movement of the medium or scattering particles inside it causes formation of dynamic speckles. Imaging of dynamic speckles with a limited exposure time results in creation of speckle pattern images of reduced contrast. The speckle contrast is a measure of relative movement: lower speckle contrast corresponds to higher movement [10].

LSCI covers a wide range of clinical applications [11], including psoriasis [12], diabetic foot [13], and cerebral blood flow monitoring during surgery (intra-operative) [14]. Operated in handheld mode, LSCI brings convenience for both patients and clinical staff [15]. However, involuntarily movements applied by test subjects or due to the handheld operations result in extra blurring of the speckle patterns. This challenge which is called movement artefact has been addressed in different ways: (1) Motion stabilization using a gimbal mount as a prevention of movement artefacts due to operator motion [16]. (2) Use of planar or spherical waves for illumination instead of conventional scrambled waves made by glass (engineered) diffusers [17]. (3) Capturing speckle patterns from an opaque surface mounted in the field-of-view (FOV) during the measurements. The perfusion measured on the opaque surface was used either to choose a number of movement-artefact-free frames [18] or to be subtracted from the perfusion values measured on tissue as a compensation of movement artefacts [19-22]. Altogether, a fundamental model that explains the underlying reason but also predicts movement artefact is missing.

Almost two decades prior to the development of LSCI, laser Doppler flowmetry had been shown to measure relative fluid flow [23]. Laser Doppler and time-varying speckle are equivalent concepts in terms of line-of-sight velocity [24]. There is a direct relation between the contrast of time-integrated speckle and power spectrum of the Doppler shifted light $[25,26]$. There are possibilities for cross-fertilization of ideas between the two concepts when it comes to differences such as modeling of multiple scattering in laser Doppler and in-plane sensitivity of speckle; therefore, there is a need to develop a unified model. In this work, we use the concept of optical Doppler effect to predict speckle contrast drop due to linear motion, and for static objects. This is a first step in modelling movement artefacts due to linear motion and rotation when measuring on objects with internal motion, such as perfused tissue. The rest of the chapter is organized as follows. Section 4.2 describes the system model and the developed theory. Section 4.3 validates the proposed theory by computer simulation. 


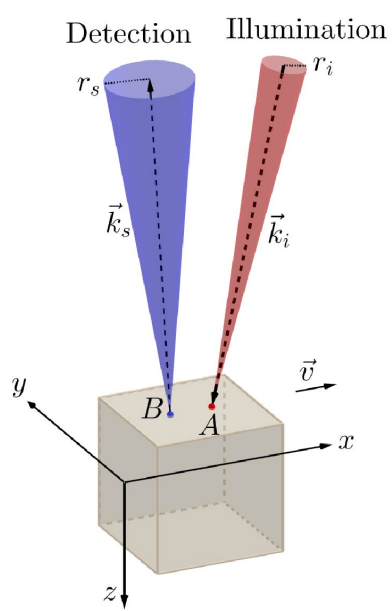

(a)

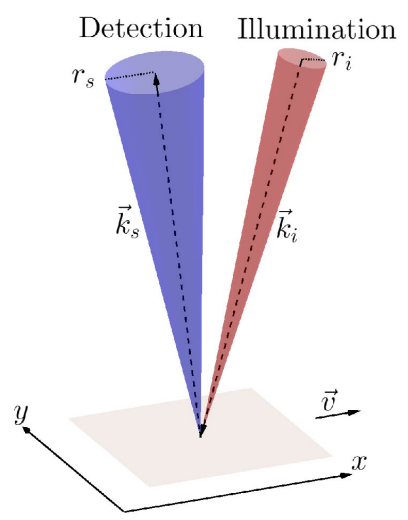

(b)

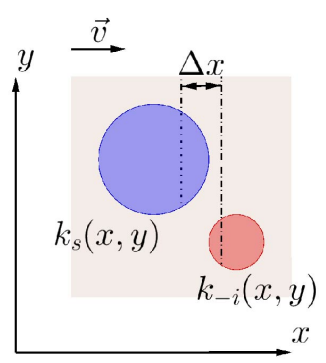

(c)

Fig. 4.1: Schematic diagram of the system model. Linear translation of a solid object along the $x-y$ plane. Illumination and detection on a solid object of (a) finite and (b) very high scattering levels. (c) Top view of the case of (b) showing the mapping of the bases of the illumination and detection cones on the object's surface.

Finally, Section 4.4 discusses the results and concludes the chapter.

\subsection{Model of the optical system and speckle generation}

\subsubsection{Optical Doppler shifts induced by translation of a solid object}

Consider a solid object of finite scattering level moving with a constant velocity $\vec{v}$ along the $x-y$ plane (see Fig. 4.1(a)). The object is illuminated by a coherent light source. For a given point $A$ on the object's surface, a cone of base radius $r_{i}$ is formed that is defined by the range of incoming wavevectors $\vec{k}_{i}$. Assuming a lens collecting scattered light at its focal point $B$ located at the object's surface, a cone of base radius $r_{s}$ defined by the range of wavevectors $\vec{k}_{s}$ will be formed. The collecting lens will conjugate the point $B$ at the imaging screen of a detector causing to observe time varying intensity $I(t)$ containing a range of frequencies that are dictated by the optical Doppler effect as [23]

$$
\omega_{D}=\vec{v} \cdot\left(\vec{k}_{i}-\vec{k}_{s}\right)
$$

The type of illumination determines the spread of $\vec{k}_{i}$ and, therefore, the size of $r_{i}$. For scrambled waves made by a glass diffuser, the $r_{i}$ takes a nonzero value. However, for spherical waves (e.g. those coming from an optical fiber tip) or plane waves, there 
is only a single $\vec{k}_{i}$ on $A$ which leads $r_{i}$ to be zero. The openness of a diaphragm located at the base of detection cone, controls the spread of $\vec{k}_{s}$ and the size of $r_{s}$ consequently. If the scattering medium is of finite scattering level, then for each detection point $B$ on the object's surface, the scattered light originally entering the medium at a range of positions around $B$ contribute to the observed Doppler shift introduced in Eq. (4.1). This range depends on the type of illumination and the optical properties of the medium.

\section{High scattering medium}

Consider the scattering medium shown in Fig. 4.1(a) to be of high scattering level such that no light penetrates into the medium (e.g. a matte surface). Then, $A$ and $B$ will coincide, and for each observation point $B$, only the incoming wavevectors to $B$ will contribute to creation of Doppler shift on the conjugated point on the detector. Now the problem narrows down to a simplified geometry as shown in Fig. 4.1(b). If the bases of illumination and detection cones are mapped on the $x-y$ plane and assuming that the angles between the normal to the surface and both $\overrightarrow{k_{-i}}$ and $\overrightarrow{k_{s}}$ is relatively small, then the two circular-shape collections for $\overrightarrow{k_{-i}}(x, y)$ and $\vec{k}_{s}(x, y)$ can be introduced as shown in Fig. 4.1(c). Note that the circular shapes only hold for relatively small tilt angles of the cones with respect to the normal on the $x-y$ plane. All the wavevectors differences indicated by $\Delta x$ will create the same Doppler shift. Moreover, in laser Doppler flowmetry and laser speckle contrast flowmetry techniques, being based on optical interferometry, the frequencies of the fluctuations are caused by the variation of the Doppler shifts but not the absolute Doppler shifts. Consequently, the absolute tilt angles of the wavevectors are not of importance but the wavevector spreads, that are modelled by $r_{i, s}$.

From Eq. (4.1), a single Doppler shift as a function of the wavevector difference can be written as

$$
\omega_{D}(\Delta x)=\frac{2 \pi V}{\lambda} l_{s-i}(\Delta x)
$$

where $V$ is absolute speed, $\lambda$ is the wavelength of the light source, $l_{s-i}($.$) is a dimen-$ sionless vector element function that depends on the spreads of $\vec{k}_{-i}(x, y)$ and $\vec{k}_{s}(x, y)$. A Doppler histogram is made by adding all the single Doppler shifts introduced in Eq. (4.2) as

$$
A\left(\frac{\omega_{D} \lambda}{2 \pi V}\right)=p_{R_{-i}}\left(\frac{\omega_{D} \lambda}{2 \pi V}\right) * p_{R_{s}}\left(\frac{\omega_{D} \lambda}{2 \pi V}\right)
$$

where $*$ denotes the convolution operator, $p_{R_{-i}}($.$) and p_{R_{s}}($.$) are probability density$ functions of the spreads of incoming and outgoing wavevectors, respectively. The 
power spectral density can be calculated as [25]

$$
P\left(\frac{\omega_{D} \lambda}{2 \pi V}\right)=A\left(\frac{\omega_{D} \lambda}{2 \pi V}\right) * A\left(\frac{\omega_{D} \lambda}{2 \pi V}\right)
$$

Then, the normalized intensity autocorrelation is calculated by

$$
g^{(2)}(\tau)=\mathscr{F}^{-1}\left\{P\left(\frac{\omega_{D} \lambda}{2 \pi V}\right)\right\}
$$

where $\mathscr{F}^{-1}\{$.$\} is the inverse Fourier transformation operator. Now, based on the$ properties of the Fourier transform [27], $g^{(2)}(\tau)$ can be calculated from $p_{R_{-i}}$ and $p_{R_{s}}$ as

$$
g^{(2)}(\tau)=\left[\mathscr{F}^{-1}\left\{p_{R_{-i}}\right\}\right]^{2}\left[\mathscr{F}^{-1}\left\{p_{R_{s}}\right\}\right]^{2}
$$

The temporal contrast of time-integrated speckle intensity is

$$
C=\sqrt{\frac{2}{T} \int_{0}^{T}\left[1-\frac{\tau}{T}\right]\left|g^{(1)}(\tau)\right|^{2} d \tau}
$$

where $g^{(1)}(\tau)=\sqrt{g^{(2)}(\tau)-1}$ is the normalized complex speckle field correlation [28] (Siegert relation). See Appendix B for the proof of Eq. (4.7) which also holds for spatial contrast of time-integrated intensity [29]. Therefore, on a highly scattering object (i.e. infinite scattering coefficient) it is possible to predict the speckle contrast drop as a result of applied $V$ when there is knowledge about $p_{R_{-i}}$ and $p_{R_{s}}$.

The density functions $p_{R_{-i}}$ and $p_{R_{s}}$ can take several possible forms of which we consider two, namely, semi-circular and Gaussian.

- For semi-circular, they take the following form

$$
p_{R_{-i, s}}\left(\frac{\omega_{D} \lambda}{2 \pi V}\right)=\frac{\lambda}{\pi^{2} V \chi_{i, s}} \sqrt{1-\left(\frac{\omega_{D} \lambda}{2 \pi V \chi_{i, s}}\right)^{2}}
$$

where $\chi_{i, s}$ are shape parameters that control the spread of $p_{R_{-i}}$ and $p_{R_{s}}$, respectively. Figure 4.2(a) represents a pair of $p_{R_{-i}}$ and $p_{R_{s}}$ with semi-circular shapes. The corresponding optical Doppler histogram is shown in 4.2(b).

Substituting Eq. (4.8) into Eq. (4.6) and performing the calculation result in

$$
g^{(2)}(\tau)=16 b_{i} b_{s}\left(\frac{J_{1}\left(\frac{\tau}{b_{i}}\right) J_{1}\left(\frac{\tau}{b_{s}}\right)}{\tau^{2}}\right)^{2}+1
$$




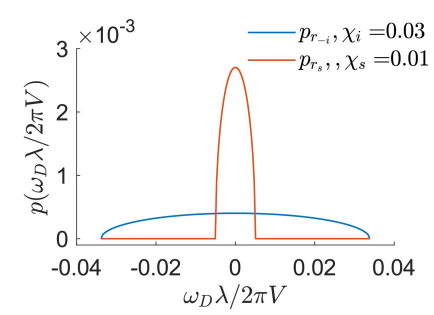

(a)

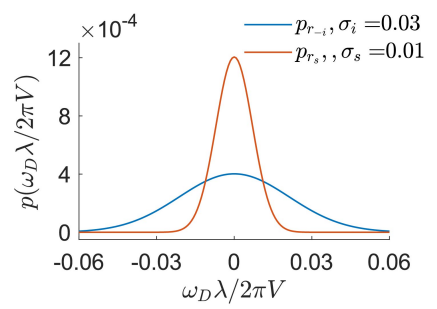

(c)

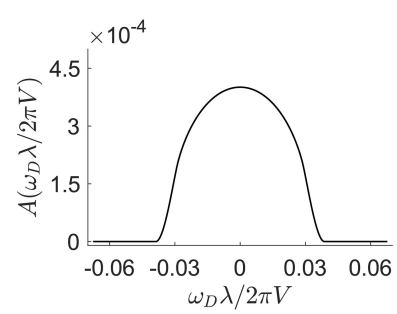

(b)

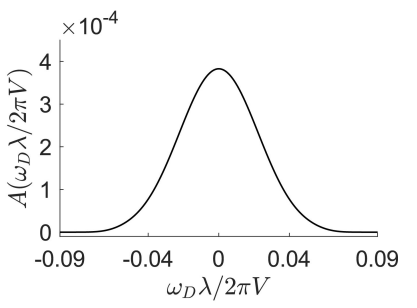

(d)

Fig. 4.2: Density functions for incoming and outgoing wavevectors. $\lambda=671 \mathrm{~nm} ; V=5 \mathrm{~mm} / \mathrm{s}$. (a) Case of semi-circular shape and (b) the associated optical Doppler histogram resulting of convolution of $p_{R_{-i}}$ and $p_{R_{s}}$. (c) Case of Gaussian shape and (d) the associated optical Doppler histogram.

where $J_{1}($.$) is the Bessel function of the first kind [30] and b_{i, s}=\lambda /\left(2 \pi V \chi_{i, s}\right)$. By substituting the calculated $g^{(1)}(\tau)$ from Eq. (4.9) into Eq. (4.7), the contrast of the time-integrated intensity takes the following form

$$
C=\sqrt{\frac{32 b_{i} b_{s}}{T} \int_{0}^{T} \frac{1}{\tau^{4}} J_{1}\left(\frac{\tau}{b_{i}}\right)^{2} \cdot J_{1}\left(\frac{\tau}{b_{s}}\right)^{2}\left[1-\frac{\tau}{T}\right]} d \tau
$$

Eq. (4.10) cannot be solved analytically; however, it can be calculated numerically.

- The Gaussian forms of the density functions $p_{R_{-i}}$ and $p_{R_{s}}$ can be written as follows

$$
p_{R_{-i, s}}\left(\frac{\omega_{D} \lambda}{2 \pi V}\right)=\exp \left(-\left(\frac{\omega_{\mathrm{D}} \lambda}{2 \pi \mathrm{V} \sigma_{\mathrm{i}, \mathrm{s}}}\right)^{2}\right)
$$

where $\sigma_{i, s}$ are the shape parameters that control the spread of $p_{R_{-i}}$ and $p_{R_{s}}$, respectively. An example of a pair of $p_{R_{-i}}$ and $p_{R_{s}}$ with Gaussian shapes and the corresponding optical Doppler histogram are shown in 4.2(c-d), respectively.

Similar to the semi-circular case, substitution of Eq.(4.11) into Eq. (4.6) results in

$$
g^{2}(\tau)=\exp \left(-\frac{1}{2} a \tau^{2}\right)+1
$$


where $a=(2 \pi V / \lambda)^{2}\left[\sigma_{i}^{2}+\sigma_{s}^{2}\right]$. Finally, the substitution of the calculated $g^{(1)}(\tau)$ from Eq. (4.12) into Eq. (4.7) results the following contrast of the timeintegrated intensity

$$
C=\sqrt{\frac{2}{T}\left[\sqrt{\frac{\pi}{2 a}} \operatorname{erf}\left(T \sqrt{\frac{a}{2}}\right)+\frac{\exp \left(-\frac{1}{2} a T^{2}\right)-1}{a T}\right]}
$$

where $\operatorname{erf}(z)=(2 / \sqrt{\pi}) \int_{0}^{z} \exp \left(-t^{2}\right) d t$ is the error function.

\section{Finite scattering medium}

In case of having a medium with finite scattering level, Eqs. (4.10) and (4.13) only hold for plane wave illumination. This is because for a finite scattering level, a single detection point $B$ (Fig. 4.1(a)) is associated with a range of light injection positions $A$. Only for plane wave illumination the illumination wavevector will be the same for all positions of injection of the light. Basically, with plane wave illumination, the observed Doppler shift will be independent of the light path ways from an arbitrary point $A$ to the point $B$.

For the cases of spherical waves, there is a single $\vec{k}_{i}$ per arbitrary point $A$, but the direction of each $\vec{k}_{i}$ depends on the location of $A$. Therefore, when illuminating with spherical or scrambled waves, information about the range of light injection positions that contribute to the detection in the observation point $B$ is required. The fluence distribution around the observation point $B$ can be obtained by running a Monte-Carlo simulation of light propagation and requires knowledge about scattering properties of the medium of interest.

\subsubsection{Simulation of time-varying speckle patterns of size 1 pixel}

For validation of the proposed theory, a time-dependent speckle complex field is generated for each pixel at location $(x, y)$ as

$$
E(x, y, t)=\frac{1}{N} \sum_{n=1}^{N} \exp \left(i\left(\omega_{n}(x, y) t+\phi_{n}(x, y)\right)\right)
$$

Here $N$ is the number of phasors set as 50 in the simulation, $i=\sqrt{-1}, \phi_{n}(x, y)$ is a uniformly distributed random number on the interval $[-\pi, \pi]$ at each position $(x, y)$ and $\omega_{n}(x, y)$ is an angular frequency picked from the optical Doppler density function $A\left(\frac{\omega_{D} \lambda}{2 \pi V}\right)$ introduced in Eq. (4.3) in a weighted manner: the higher the amplitude of frequency in $A\left(\frac{\omega_{D} \lambda}{2 \pi V}\right)$, the higher the chance of selecting that frequency as $\omega_{n}(x, y)$. In the dynamic speckle pattern obtained in this manner each speckle occupies one pixel. 


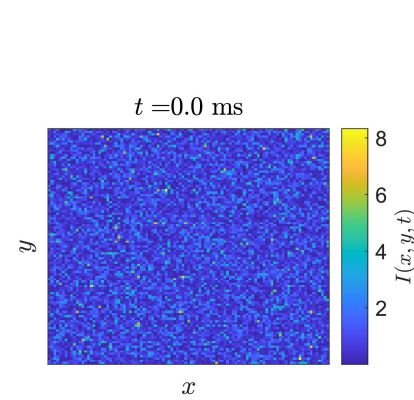

(a)

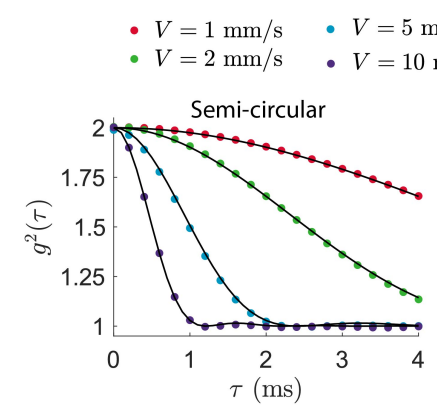

(b)
- Theoretical

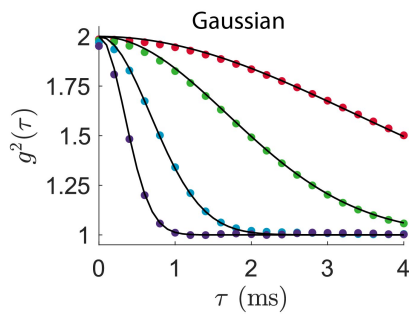

(c)

Fig. 4.3: Generation of time-varying speckle patterns. (a) A frame of speckle intensity. Normalized intensity auto-correlations with (b) semi-circular and (c) Gaussian forms of the wavevector density functions. In (b-c), a comparison between results of simulated speckle patterns and theory is shown.

Figure C.1(a) shows a simulated frame of the absolute field of size $100 \times 100 \mathrm{px}$. The corresponding speckle intensity is calculated as

$$
I(x, y, t)=|E(x, y, t)|^{2}
$$

The absolute field of a fully developed speckle pattern follows a Rayleigh distribution with the following form [31] (see Fig. C.1(b))

$$
p_{E}(E)=\frac{E}{\sigma^{2}} \exp \left(-\frac{E^{2}}{2 \sigma^{2}}\right)
$$

where $\sigma=\sigma_{R}=\sigma_{I}$ is the standard deviation of either real or imaginary part of the complex field. In addition, a fully developed speckle pattern has a negative exponential intensity $\left(I=|E|^{2}\right)$ distribution with the following form (see Fig. C.1(c))

$$
p_{I}(I)=\frac{1}{\bar{I}} \exp \left(-\frac{I}{\bar{I}}\right)
$$

where $\bar{I}=2 \sigma^{2}$ is the mean intensity. The time-integrated speckle intensity is

$$
I_{T}(x, y)=\int_{0}^{T} I(x, y, t) d t
$$

where $T$ denotes the exposure time. Lastly, the spatial contrast of the time-integrated speckle intensity is calculated as

$$
C=\frac{\sigma_{I_{T}}(x, y)}{\overline{I_{T}}(x, y)}
$$

where $\sigma_{I_{T}}(x, y)$ and $\bar{I}_{T}(x, y)$ represent the spatial standard deviation and mean of $I_{T}(x, y)$, respectively. 


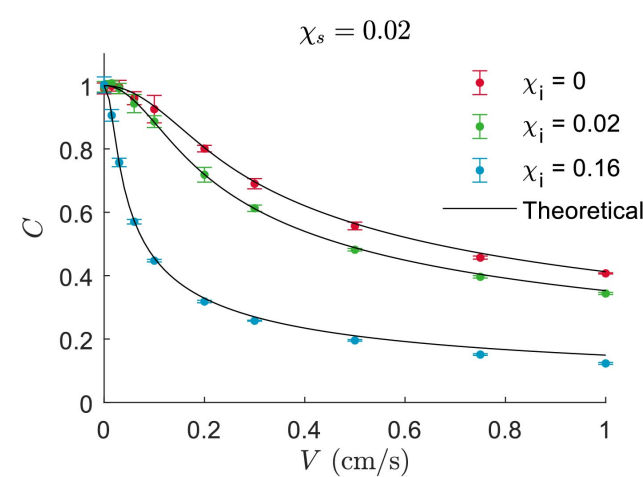

(a)

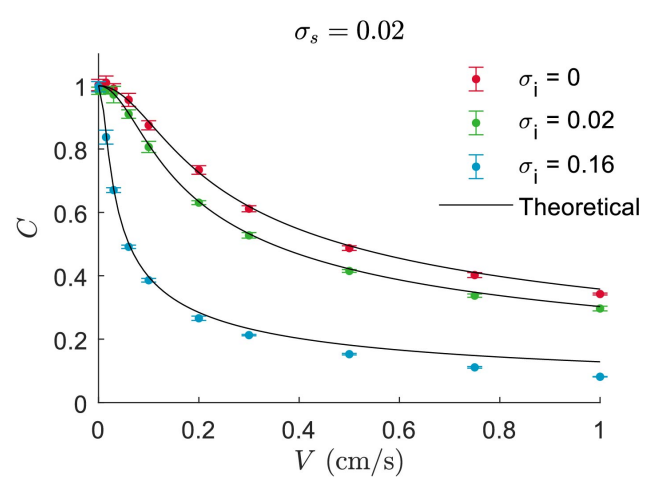

(b)

Fig. 4.4: Speckle contrast versus the applied translational speed. The density functions for the wavevectors are (a) semi-circular and (b) Gaussian. $\left\{\chi_{i}, \sigma_{i}\right\}=0$ simulate spherical or planar waves, while $\left\{\chi_{i}, \sigma_{i}\right\}>0$ simulate scrambled waves.

\subsection{Results}

A simulated speckle pattern introduced in Eq. (4.15) is shown in Fig. 4.3(a) which is made by a pair of semi-circular density functions introduced in Eq. (4.8) with $\chi_{i}=0.03$ and $\chi_{s}=0.01$. Visualization 4.1 and Visualization 4.2 show the speckle intensity frames during an episode of $10 \mathrm{~ms}$ for $V=2 \mathrm{~mm} / \mathrm{s}$ and $10 \mathrm{~mm} / \mathrm{s}$, respectively, and the corresponding $g^{2}(\tau)$ numerically calculated from Eq. (4.9). It can be seen that the higher the velocity the more dynamic the speckle patterns are and the more rapid the decay of the intensity correlation.

To demonstrate that the temporal aspect has been implemented correctly, normalized intensity correlation functions $\left(g^{2}(\tau)\right)$ are also calculated from simulations and compared with the theory. Fig. 4.3(b-c) depicts intensity correlation functions for the pairs of semi-circular and Gaussian $\left(\sigma_{i}=0.03\right.$ and $\left.\sigma_{s}=0.01\right)$ wave vector density functions, respectively, for a range of applied velocities. The theoretical $g^{2}(\tau)$ curve of the Gaussian pair is calculated from Eq. (4.12). It can be observed that for the both semi-circular and Gaussian cases, the simulated values are in agreement with the theoretical predictions.

In Fig. 4.4(a), the spatial speckle contrast is shown versus the applied translational velocity for semi-circular density functions introduced in Eq. (4.8). The theoretical curves are calculated based on Eq. (4.10) while the simulated points are obtained by Eq. (4.19). Here, the detection system (modeled by $\chi_{s}$ ) is kept constant, while three different values for the spread of incoming wavevectors (i.e. $\chi_{i}$ ) are realised. $\chi_{i}=0$ simulates planar or spherical waves, while $\chi_{i}>0$ simulates scrambled waves such that a larger $\chi_{i}$ corresponds to a wider area on a diffuser's surface being illuminated.

Similarly, in Fig. 4.4(b) the spatial speckle contrast is plotted versus the applied 
translational velocity for Gaussian density functions shown in Eq. (4.11). The theoretical curves are obtained by Eq. (4.13). The effect of illumination types is simulated here by changing $\sigma_{i}$ while keeping $\sigma_{s}$ unchanged.

\subsection{Discussion}

A model has been developed with the purpose of predicting movement artefacts caused by translation in handheld laser speckle contrast perfusion imaging. Our model is based on the optical Doppler shift distributions associated with the range of wave vectors for illumination and detection. It is shown that the speckle contrast drop which is a measure of movement artefacts depends on the applied translational velocity, the type of illumination, and the geometry of detection. Equation (4.7), suggests that the speckle contrast drop is independent of speckle boiling, shifting or a combination of both. What dictates the speckle contrast drop is the spatial one-point auto-correlation of the field. This statement is not concluded by the developed model but it is purely originated from the definition of speckle contrast. The importance of this statement is that it facilitates further development of the model.

Two forms of semi-circular and Gaussian are proposed for the density functions of the incoming and outgoing wavevector sets. Analytical expressions for the contrast of time-integrated intensity for the two types of density functions have been derived that can be calculated numerically. Therefore, a connection has been established between the contrast measured in a typical LSCI and the optical Doppler shift. The model is validated by simulation of speckle patterns where an agreement has been found between the simulated and the theoretical values for the cases of semi-circular and Gaussian density functions (see Fig. 4.4).

What has been investigated in this chapter includes the combination of (1) finite scattering level and the plane wave illumination and (2) high scattering level and illumination with plane waves, spherical waves and scrambled waves. In an in-vivo LSCI measurement, the subject of interest is human skin which is of finite scattering level. Therefore, as a future work, it is essential to combine the developed model with a Monte-Carlo simulation of light propagation in order to calculate the fluence distribution around each detection point and predict the speckle contrast drop. It is also of interest to account for a non-static medium in which some extent of movements such as Brownian motion is introduced. In that case, the proposed model should be adjusted and the validation simulation and experiments should be performed. 


\section{References}

[1] A. Fercher and J. D. Briers, "Flow visualization by means of single-exposure speckle photography", Optics communications 37, 326-330 (1981).

[2] J. D. Briers, "Laser speckle contrast imaging for measuring blood flow.", Optica Applicata 37 (2007).

[3] D. A. Boas and A. K. Dunn, "Laser speckle contrast imaging in biomedical optics", Journal of biomedical optics 15, 011109 (2010).

[4] J. Senarathna, A. Rege, N. Li, and N. V. Thakor, "Laser speckle contrast imaging: theory, instrumentation and applications", IEEE reviews in biomedical engineering 6, 99-110 (2013).

[5] S. C. Gnyawali, K. Blum, D. Pal, S. Ghatak, S. Khanna, S. Roy, and C. K. Sen, "Retooling laser speckle contrast analysis algorithm to enhance non-invasive high resolution laser speckle functional imaging of cutaneous microcirculation", Scientific reports 7, 41048 (2017).

[6] M. Hultman, M. Larsson, T. Strömberg, and I. Fredriksson, "Real-time video-rate perfusion imaging using multi-exposure laser speckle contrast imaging and machine learning”, Journal of Biomedical Optics 25, 116007 (2020).

[7] D. D. Duncan and S. J. Kirkpatrick, "Can laser speckle flowmetry be made a quantitative tool?", JOSA A 25, 2088-2094 (2008).

[8] S. S. Kazmi, E. Faraji, M. A. Davis, Y.-Y. Huang, X. J. Zhang, and A. K. Dunn, "Flux or speed? examining speckle contrast imaging of vascular flows", Biomedical optics express 6, 2588-2608 (2015).

[9] A. Nadort, K. Kalkman, T. G. Van Leeuwen, and D. J. Faber, "Quantitative blood flow velocity imaging using laser speckle flowmetry", Scientific reports 6, 25258 (2016).

[10] C. Boudoux, Fundamentals of biomedical optics: From light interactions with cells to complex imaging systems (Pollux) (2017).

[11] W. Heeman, W. Steenbergen, G. M. van Dam, and E. C. Boerma, "Clinical applications of laser speckle contrast imaging: a review", Journal of biomedical optics 24, 080901 (2019).

[12] M. A. Mikhailova, E. V. Potapova, A. K. Koroleva, D. D. Stavtsev, N. B. Margaryants, N. Y. Yakushkina, and A. V. Dunaev, "A multimodal approach to monitoring the state of microvasculature in patients with psoriasis in the course of treatment", in Tissue Optics and Photonics, edited by V. V. Tuchin, W. C. P. M. Blondel, and Z. Zalevsky, volume 11363, 189 - 193, International Society for Optics and Photonics (SPIE) (2020).

[13] O. A. Mennes, M. Selles, J. J. van Netten, J. G. van Baal, W. Steenbergen, and R. H. Slart, "Semi-automatic tracking of laser speckle contrast images of microcirculation in diabetic foot ulcers", Diagnostics 10, 1054 (2020). 
[14] A. Mangraviti, F. Volpin, J. Cha, S. I. Cunningham, K. Raje, M. J. Brooke, H. Brem, A. Olivi, J. Huang, B. M. Tyler, et al., "Intraoperative laser speckle contrast imaging for real-time visualization of cerebral blood flow in cerebrovascular surgery: Results from pre-clinical studies", Scientific reports 10, 1-13 (2020).

[15] R. Farraro, O. Fathi, and B. Choi, "Handheld, point-of-care laser speckle imaging", Journal of biomedical optics 21, 094001 (2016).

[16] B. Lertsakdadet, C. Dunn, A. Bahani, C. Crouzet, and B. Choi, "Handheld motion stabilized laser speckle imaging", Biomedical optics express 10, 5149-5158 (2019).

[17] A. Chizari, T. Knop, W. Tsong, S. Schwieters, and W. Steenbergen, "Influence of wavefront types on movement artefacts in handheld laser speckle contrast perfusion imaging", OSA Continuum 4, 1875-1888 (2021).

[18] B. Lertsakdadet, B. Y. Yang, C. E. Dunn, A. Ponticorvo, C. Crouzet, N. Bernal, A. J. Durkin, and B. Choi, "Correcting for motion artifact in handheld laser speckle images", Journal of biomedical optics 23, 036006 (2018).

[19] L. Omarjee, I. Signolet, A. Humeau-Heutier, L. Martin, D. Henrion, and P. Abraham, "Optimisation of movement detection and artifact removal during laser speckle contrast imaging”, Microvascular research 97, 75-80 (2015).

[20] S. Bahadori, T. Immins, and T. W. Wainwright, "A novel approach to overcome movement artifact when using a laser speckle contrast imaging system for alternating speeds of blood microcirculation", JoVE (Journal of Visualized Experiments) 1, e56415 (2017).

[21] G. Mahé, P. Rousseau, S. Durand, S. Bricq, G. Leftheriotis, and P. Abraham, "Laser speckle contrast imaging accurately measures blood flow over moving skin surfaces", Microvascular research 81, 183-188 (2011).

[22] G. Mahé, P. Abraham, A. Le Faucheur, A. Bruneau, A. Humeau-Heurtier, and S. Durand, "Cutaneous microvascular functional assessment during exercise: a novel approach using laser speckle contrast imaging", Pflügers Archiv-European Journal of Physiology 465, 451-458 (2013).

[23] Y. Yeh and H. Cummins, "Localized fluid flow measurements with an he-ne laser spectrometer", Applied Physics Letters 4, 176-178 (1964).

[24] J. D. Briers, "Laser Doppler and time-varying speckle: a reconciliation", JOSA A 13, 345-350 (1996).

[25] I. Fredriksson and M. Larsson, "On the equivalence and differences between laser doppler flowmetry and laser speckle contrast analysis", Journal of Biomedical Optics 21, 126018 (2016).

[26] M. J. Draijer, E. Hondebrink, M. Larsson, T. G. van Leeuwen, and W. Steenbergen, "Relation between the contrast in time integrated dynamic speckle patterns and the power spectral density of their temporal intensity fluctuations", Optics express 18, 21883-21891 (2010). 
[27] J. G. Proakis, Digital communications, 2nd edition (McGraw-Hill New York) (1989).

[28] L. Mandel, "Fluctuations of photon beams and their correlations", Proceedings of the Physical Society (1958-1967) 72, 1037 (1958).

[29] J. W. Goodman, Statistical optics (John Wiley \& Sons) (2015).

[30] F. Bowman, Introduction to Bessel functions, Dover books on mathematics (Dover, New York, NY) (1958).

[31] J. W. Goodman, Speckle phenomena in optics: theory and applications (Roberts and Company Publishers) (2007). 
Appendices 



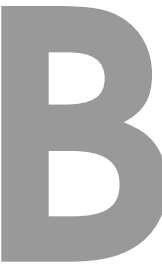

\section{Contrast of the time-integrated intensity for a fully dynamic speckle}

The equation that connects the contrast of fully dynamic speckle patterns to the integration of the field or intensity correlation is not clearly explained in the literature. The assumption is that all light undergoes a Doppler shift and the resultant speckle patterns therefore do not have a static part. Here, we employ the theory of Goodman [29] on high-order coherence of polarized thermal light (i.e. electromagnetic radiations emitted from a light source as a consequence of its temperature) to calculate the temporal speckle contrast. Since the subject speckle patterns are fully developed, they share the same statistics in space and time. Therefore, the result of the analysis is nearly identical for integrals over space in order to calculate the spatial speckle contrast. Consider the instantaneous intensity $I(x, y, z, t)$ of a complex field $E(x, y, z, t)$ at a point $P(x, y, z)$ in space. The observed time-integrated intensity on a detector is

$$
I_{T}(t)=\int_{t-T}^{t} I(\xi) d \xi
$$

with $T$ the exposure time. Since the thermal light is an ergodic random process (and therefore stationary), the statistics of $I_{T}(t)$ is independent of the observation time, allowing us to write

$$
I_{T}=\int_{-\frac{T}{2}}^{\frac{T}{2}} I(\xi) d \xi
$$


The expected (i.e. mean) value of the time-integrated intensity is

$$
\begin{aligned}
\overline{I_{T}} & =\mathbb{E}\left[\int_{-\frac{T}{2}}^{\frac{T}{2}} I(\xi) d \xi\right] \\
& =\int_{-\frac{T}{2}}^{\frac{T}{2}} \mathbb{E}[I(\xi)] d \xi \\
& =\bar{I} T .
\end{aligned}
$$

The variance of the time-integrated intensity is

$$
\sigma_{I_{T}}^{2}=\mathbb{E}\left[I_{T}^{2}\right]-{\overline{I_{T}}}^{2} .
$$

The first term (the average of the square intensity) has the form

$$
\begin{aligned}
\mathbb{E}\left[I_{T}^{2}\right] & =\mathbb{E}\left[\iint_{-\frac{T}{2}}^{\frac{T}{2}} I(\xi) I(\eta) d \xi d \eta\right] \\
& =\iint_{-\frac{T}{2}}^{\frac{T}{2}} \mathbb{E}[I(\xi) I(\eta)] d \xi d \eta \\
& =\iint_{-\frac{T}{2}}^{\frac{T}{2}} \Gamma_{I}(\xi-\eta) d \xi d \eta .
\end{aligned}
$$

The function $\Gamma_{I}(\tau)$ is the autocorrelation function of the instantaneous intensity that is defined as

$$
\begin{aligned}
\Gamma_{I}(\tau) & =\mathbb{E}\left[E(t) E^{*}(t) E(t+\tau) E^{*}(t+\tau)\right] \\
& =\lim _{x \rightarrow+\infty} \frac{1}{x} \int_{0}^{x} I(t) I(t+\tau) d t
\end{aligned}
$$

where $*$ represents the complex-conjugate operator and $\Gamma_{I}(\tau) / \bar{I}^{2}=g^{2}(\tau)$. Equation (B.5) can be rewritten as

$$
\mathbb{E}\left[I_{T}^{2}\right]=\iint_{-\infty}^{\infty} \operatorname{rect}\left(\frac{\xi}{T}\right) \operatorname{rect}\left(\frac{\eta}{T}\right) \Gamma_{I}(\xi-\eta) d \xi d \eta,
$$

where $\operatorname{rect}(x)=1$ for $|x| \leq \frac{1}{2}$ and zero otherwise. By replacing the variables $\xi=\tau+t$ and $\eta=t$ (and thus $d \xi=d \tau$ and $d \eta=d t$ ) we have

$$
\begin{aligned}
\mathbb{E}\left[I_{T}^{2}\right] & =\iint_{-\infty}^{\infty} \operatorname{rect}\left(\frac{\tau+t}{T}\right) \operatorname{rect}\left(\frac{t}{T}\right) \Gamma_{I}(\tau) d \tau d t \\
& =\int_{-\infty}^{\infty}[\underbrace{\int_{-\infty}^{\infty} \operatorname{rect}\left(\frac{\tau+t}{T}\right) \operatorname{rect}\left(\frac{t}{T}\right) d t}_{f(\tau)}] \Gamma_{I}(\tau) d \tau .
\end{aligned}
$$


The functions $\operatorname{rect}\left(\frac{\tau+t}{T}\right)$ and $\operatorname{rect}\left(\frac{t}{T}\right)$ return unity for $-\frac{T}{2}-|\tau| \leq t \leq \frac{T}{2}-|\tau|$ and $-\frac{T}{2} \leq t \leq \frac{T}{2}$, respectively, and zero otherwise. The intersection of the last two intervals would mean $-\frac{T}{2} \leq t \leq \frac{T}{2}-|\tau|$, that results in

$$
\begin{aligned}
f(\tau) & =\int_{-\frac{T}{2}}^{\frac{T}{2}-|\tau|} d t \\
& =T-|\tau| .
\end{aligned}
$$

Substituting Eq. (B.9) into Eq. (B.8) and considering $-\frac{T}{2} \leq \frac{T}{2}-|\tau|$, we have

$$
\mathbb{E}\left[I_{T}^{2}\right]=\int_{-T}^{T}[T-|\tau|] \Gamma_{I}(\tau) d \tau
$$

The autocorrelation function $\Gamma(\tau)$ introduced in Eq. (B.6) under the assumption of a Gaussian random process can be expressed as [28]

$$
\Gamma_{I}(\tau)=\bar{I}^{2}\left[1+\left|g^{1}(\tau)\right|^{2}\right]
$$

where $g^{1}(\tau)$ is the complex degree of coherence of the light, also known as the normalized complex field correlation with the following definition [31]

$$
g^{1}(\tau)=\frac{\mathbb{E}\left[E(t+\tau) E^{*}(t)\right]}{\sqrt{\mathbb{E}\left[|E(t+\tau)|^{2}\right] \mathbb{E}\left[|E(t)|^{2}\right]}} .
$$

Now, substitution of Eq. (B.11) into Eq. (B.10) and further simplification yields the result

$$
\mathbb{E}\left[I_{T}^{2}\right]=\bar{I}^{2}\left[\int_{-T}^{T}[T-|\tau|]\left|g^{1}(\tau)\right|^{2} d \tau+T^{2}\right] .
$$

Finally, the temporal speckle contrast is

$$
\begin{aligned}
C & =\sqrt{\frac{\sigma_{I_{T}}^{2}}{\bar{I}_{T}^{2}}} \\
& =\sqrt{\frac{1}{T} \int_{-T}^{T}[T-|\tau|]\left|g^{1}(\tau)\right|^{2} d \tau} \\
& =\sqrt{\frac{2}{T} \int_{0}^{T}\left[1-\frac{\tau}{T}\right]\left|g^{1}(\tau)\right|^{2} d \tau}
\end{aligned}
$$


In a single scattering regime and assuming Browning motion of the scattering particles, the normalized field correlation function has a negative exponential form

$$
g^{1}(\tau)=e^{-\frac{|\tau|}{\tau_{c}}}
$$

with $\tau_{c}$ the correlation time, that is the time it takes for the correlation function to reach $1 / e$ of its maximum value. Substituting Eq. (B.15) into Eq. (B.14) and calculating the integration will result

$$
C=\sqrt{\frac{\tau_{c}}{T}+\frac{\tau_{c}^{2}}{2 T^{2}}\left(e^{-\frac{2 T}{\tau_{c}}}-1\right)},
$$

which is the conventional model for laser speckle contrast imaging (LSCI) [3, 7]. 


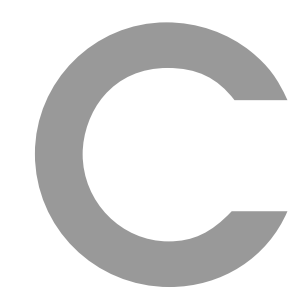

\section{Supplementary information}

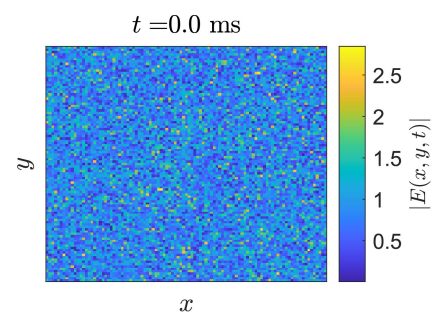

(a)

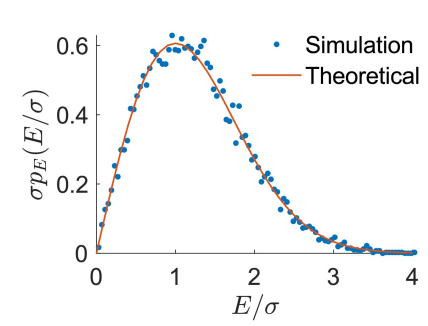

(b)

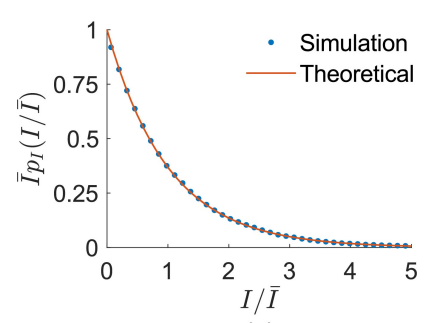

(c)

Fig. C.1: Simulation of fully dynamic speckle patterns. (a) A frame of speckle absolute field. Normalized probability density functions (PDFs) of the (b) absolute field and (c) intensity as a comparison between results of simulated speckle patterns and theory. 


\section{Handheld versus mounted laser speckle contrast perfusion imaging demonstrated in psoriasis lesions * $\dagger$}

Enabling handheld perfusion imaging would drastically improve the feasibility of perfusion imaging in clinical practice. Therefore, we examine the performance of handheld laser speckle contrast imaging (LSCI) measurements compared to mounted measurements, demonstrated in psoriatic skin. A pipeline is introduced to process, analyze and compare data of 11 measurement pairs (mounted-handheld LSCI modes) operated on 5 patients and various skin locations. The on-surface speeds (i.e. speed of light beam movements on the surface) are quantified employing mean separation (MS) segmentation and enhanced correlation coefficient maximization (ECC). The average on-surface speeds are found to be 8.5 times greater in handheld mode compared to mounted mode. Frame alignment sharpens temporally averaged perfusion maps, especially in the handheld case. The results show that after proper post-processing, the handheld measurements are in agreement with the corresponding mounted measurements on a visual basis. The absolute movement-induced difference between

${ }^{*}$ Chizari, A. ${ }^{+}$, Schaap, M.J. ${ }^{+}$, Knop, T., Boink, Y.E., Seyger, M. and Steenbergen, W. Handheld versus mounted laser speckle contrast perfusion imaging demonstrated in psoriasis lesions. Scientific Reports, 11, 16646 (2021). ${ }^{+}$These authors contributed equally. https://doi.org/10.1038/s41598-02196218-6

${ }^{\dagger}$ The development of the user interface for data acquisition and analysis; conducting the in-vitro phantom experiments; and performing the data analysis are part of the present thesis. Conducting the experiments on psoriasis patients and carrying out the statistical analysis are due to M.J. Schaap. 
mounted-handheld pairs after the background correction is $16.4 \pm 9.3 \%$ (mean \pm std, $n=11$ ), with an absolute median difference of $23.8 \%$. Realization of handheld LSCI facilitates measurements on a wide range of skin areas bringing more convenience for both patients and medical staff.

\subsection{Introduction}

The microcirculation in the skin is responsible for delivering oxygen and nutrients to the tissue and evaluation of the microvasculature provides insights regarding tissue viability and health. Microcirculatory perfusion imaging has the potential to provide valuable information on the microvasculature and has gained attention in the past years [1-4]. Examples of medical applications of perfusion imaging include psoriasis [5-7], burn wound depth assessment [8] and postoperative monitoring [9]. Among the optical perfusion imaging techniques, laser speckle contrast imaging (LSCI) has been a point of interest in the past three decades since it is non-invasive, affordable and compact [10]. In this imaging modality, the skin surface is illuminated by coherent laser light. Due to the roughness of the surface and multiple light scattering inside the tissue, an interference pattern is observed on the camera detector called optical speckle. Blurred speckle patterns are created by movements of red blood cells (RBCs) within a certain exposure time. Their contrast depends on the movement: a larger movement causes lower speckle contrast [11]. The speckle contrast distribution can be transformed into a perfusion map of the illuminated tissue.

The sensitivity of optical speckles to movements of other sources, such as involuntarily movements of the patients, introduces challenges during (handheld) measurements [12]. Several attempts to reduce the effect of these movements have been described in literature. Using an adjacent opaque surface, Mahe et al. [13] obtained mounted LSCI data over a moving skin surface as well as during exercise [14] and compensated the occurred movement artefacts. Their protocol was later optimized by Omarjee et al. [15]. Due to the need to make the LSCI system portable, which is especially important in a clinical setting, Lertsakdadet et al. [16] proposed a handheld LSCI device with a fiducial marker as an indicator of movement artefacts as well as introducing a motion stabilized version of this device using a motorized gimbal mount [17]. To date, all approaches are based on attachment of an opaque surface on the tissue for frame alignment and accounting for movement artefacts. The room for improvement is to find alternatives for such opaque surfaces so that they can be removed.

In a previous work, we measured movements of the LSCI system during handheld experiments using an electromagnetic (EM) tracker. We analyzed movement artefacts in response to on-surface beam speed, that is the speed of the light beam on the surface caused by translation and rotation of the probe, and wavefront tilting for media with 
various scattering levels [18]. In addition, we explored the influence of wavefront types on movement artefacts employing three types of illumination, namely scrambled, spherical and planar wavefronts [19]. Results of handheld measurements showed that on average spherical and planar wavefronts cause less drop in the speckle contrast compared to a scrambled wavefront while measuring on a tissue mimicking static phantom.

In this work, we explore the validity of handheld measurements compared to mounted measurements (as the golden standard) demonstrated in psoriatic skin. We chose to use psoriasis lesions since it is well known that microvasculature changes and angiogenesis are important in psoriasis [20,21]. Additionally, perfusion inhomogeneity is present in psoriasis plaques. This results in so called hot spots and cold spots [5, 22], that provides valuable perfusion maps for comparison of mounted and handheld measurements. A methodology is proposed to post-process the acquired raw speckle frames under the presence of small natural movements of patients and operators, and to compute a representative perfusion map per experiment. Moreover, the theoretical model for assigning perfusion values to the measured speckle contrast is examined in-vitro and in-vivo. The aim is to study the proportionality of estimated perfusion and the applied speed.

\subsection{Methods and materials}

\subsubsection{Handheld perfusion imager (HAPI)}

A handheld LSCI device was designed to be utilized in a clinical research setting, performing both red-green-blue (RGB) and perfusion imaging. Figure 5.1 illustrates an overview of the experimental setup.

For perfusion imaging, a coherent and continuous wave single longitudinal mode laser (CNI MSL - FN - 671) with a wavelength of 671 and output power of $300 \mathrm{~mW}$ was used with a coherence length $>50 \mathrm{~m}$. The laser beam was attenuated by an absorptive filter of 0.2 optical density (Thorlabs NE02A) with a non-perpendicular surface to the beam, preventing direct back-reflection into the laser. The laser beam was then directed to an olympus plan achromat microscope objective (Thorlabs, RMS10X), that was mounted on a three axis stage (Thorlabs Nanomax 300), via broadband dielectric mirrors (Thorlabs BB1 - E02). The three axis stage was used to focus the light into a single mode optical fiber (Thorlabs P1 - 630A - FC - 5) and an FC/APC connection to prevent back-reflections to the laser head. The distal end of the optical fiber is attached to the handheld probe where its outgoing beam has been diverged using a mounted plano-concave lens (Qioptiq, $\mathrm{N}-\mathrm{BK} 7$ ) of focal distance $-6 \mathrm{~mm}$, diameter of $6 \mathrm{~mm}$ and was located $25 \mathrm{~mm}$ away from the fiber tip. This leads to a diverging laser beam with a spherical wavefront. The distance from the light source and camera sensors to the tissue surface has been set to $40 \mathrm{~cm}$, although it varied 

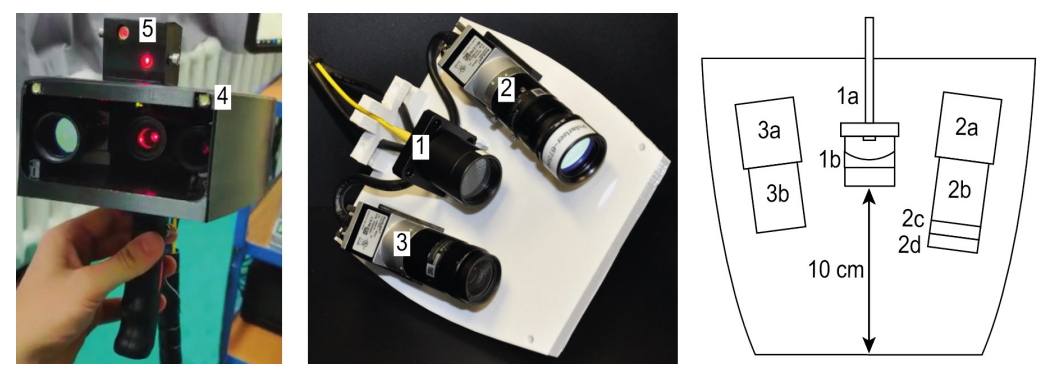

Fig. 5.1: Experimental setup of the handheld perfusion imager (HAPI). Left, a front view of the handheld probe. Middle, handheld probe without a cap (reprinted from [18]). Right, schematic drawing of the handheld probe. 1, Fiber tip (1a) and lens for laser beam expansion (1b). 2, Set of monochromatic camera (2a), objective lens (2b), bandpass filter (2c) and linear polarizer (2d). 3, Set of RGB camera (3a) and objective lens (3b). 4, Power light emitting diodes (LEDs) for white-light illumination. 5, Targeting lasers. The total weight of the handheld probe is $995 \pm 25 \mathrm{gr}$.

slightly during handheld operations. The measured beam width, i.e. the radial distance at which the intensity decreases by a factor of $1 / e^{2}$ of its maximum at the center of illumination, for this system is approximately $8 \mathrm{~cm}$. Also, the full width at half max (FWHM) of the beam intensity is measured as $8.8 \mathrm{~cm}$. Previously, we demonstrated that, using a spherical wavefront, movement artefacts due to the rotation of the probe could be reduced in comparison to the use of a conventional engineered diffuser which generates a scrambled wavefront [19].

To ensure eye safety, the following precautions are taken: (1) use of a visible wavelength (i.e. red) compared to near infrared; (2) training of the operator of the handheld probe prior to the study. To allow or block laser illumination on the tissue, a motorized shutter was made by a translational stage (Zaber, T - LSM050A). Two cross-line laser modules with optical power $5 \mathrm{~mW}$ and operating wavelength $650 \mathrm{~nm}$, each driven by $25 \mathrm{~mA}$ electrical current, were used to illuminate the boundaries of the imaging fields-of-view FOVs. This aided the operator in targeting the imaging regions during perfusion imaging. The RGB camera was not of help for targeting the imaging regions during perfusion measurement, because the white light illumination was switched off.

To record the speckle intensity patterns for the patient measurements, a monochrome camera (Basler acA2040 55um USB3) was used that operated at a frame rate of $30 \mathrm{~Hz}$, imaging depth of 8 bits, gain of $10 \mathrm{~dB}$, exposure time of $10 \mathrm{~ms}$ and frame size of $1536 \mathrm{px} \times 2048 \mathrm{px}$. The camera objective (FUJINON HF16XA $-5 \mathrm{M}$ ) had a focal length of $16 \mathrm{~mm}$ and an f-number of $F / 8$ in order to obtain the optimum point for (1) the detected light intensity to have the highest dynamic range for computation of speckle contrast and (2) the speckle size to meet the Nyquist criterion [23]. The magnification of the imaging system was measured as $11.9 \mathrm{px} / \mathrm{mm}$ resulting in an 
FOV of $12.9 \times 17.2 \mathrm{~cm}^{2}$. To reduce noise from background light, a hard coated bandpass interference filter (Edmund Optics) of wavelength $675 \pm 12.5 \mathrm{~nm}$ was mounted on the objective as well as turning off the general illumination of the room during each measurement. A linear polarizer (Thorlabs LPNIRE $100-$ B) with a polarization direction perpendicular to that of the laser beam was used in the imaging system to minimize specular reflections and to increase measurable speckle contrast.

For making RGB images, a color camera(Basler acA1920 - 40uc USB3) was used which operated at the imaging depth of 8 bits, gain of $12 \mathrm{~dB}$, exposure time of $10 \mathrm{~ms}$ and frame size of $1200 \mathrm{px} \times 1920 \mathrm{px}$. Its objective (FUJINON HF12XA $-5 \mathrm{M}$ ) had a $12 \mathrm{~mm}$ focal length and worked with the fully open diaphragm. With a magnification of $5.6 \mathrm{px} / \mathrm{mm}$ the obtained FOV was $21.4 \times 34.3 \mathrm{~cm}^{2}$. Two power light emitting diodes (LEDs) of each $1 \mathrm{~W}$ maximum electrical power and driven by $90 \mathrm{~mA}$ electrical current were used as illumination sources during the imaging.

\subsubsection{Study population}

Five adult psoriasis patients ( 2 female and 3 male) participated in this study. In total, eleven pairs of mounted and handheld measurements were carried out. The time interval between each measurement pair was minimized to maximally 9 minutes (Supplementary Tables 5.2-5.12). In order to minimize external influences on the skin perfusion level, patients were asked to refrain from heavy physical activities, scratching of the skin, drinking caffeine or smoking for at least 30 minutes prior to each measurement. The in-vivo evaluation of the perfusion estimation model mentioned in Subsection 5.3.2 was performed on a healthy volunteer. Informed consent was obtained from all participants before enrollment. Utilization of the HAPI and the study protocol was approved by the ethics committee of the region of Arnhem-Nijmegen and the Radboud university medical center, Nijmegen, the Netherlands (NL69174.091.19).

\subsubsection{Measurement protocol}

The laser source for perfusion imaging was turned on at least 15 minutes prior to each measurement to warm up. A calibration cap was placed in front of the handheld probe to take a snapshot of a Delrin plate (Polyoxymethylene) and a scattering suspension (Perimed, PF 1001 Refill Motility Standard) located at its working distance. The PF 1001 Refill Motility Standard is a colloidal suspension of polystyrene particles. For a particle diameter of $320 \mathrm{~nm}$, the concentration of the suspension would be $1.2 \times 10^{12}$ per cube centimeter [24]. The average speckle intensity on the Delrin plate was checked via a custom-made user-interface in MATLAB R2019b and maximized by manually adjusting the three axis stage on which the microscope objective directs laser light into the optical fiber. The obtained average intensities $\bar{I}$ are between $13.5-21.9$ out of 255 . The eye safety and the maximum tolerable optical power through a single 
mode optical fiber are two limiting factors in obtaining a higher average intensity level in the imaging plane. Therefore, the camera gain was used to enhance the detected intensity level. Use of a bandpass filter resulted in a high signal-to-noise-ratio (SNR) which facilitated detection of suggestive heartbeat patterns by monitoring temporal fluctuations of spatial speckle contrast on a lesion. Moreover, temporal averaging of the perfusion maps ensured obtaining a clear difference between areas with low and high perfusion values. The speckle contrasts of regions selected on the Delrin plate and scattering suspension were in the ranges of $C_{\mathrm{s}}=0.64-0.94$ and $C_{\mathrm{d}}=0.16-0.24$, respectively (Supplementary Tables 5.2-5.12). This calibration data was not used for adjusting data but for checking the stability of the system.

Black marker dots (Edding 400, $1 \mathrm{~mm}$, permanent marker) were placed by a physician on the clinical psoriatic lesion border to provide a reference of the clinically visible lesion borders on the perfusion images. Depending on the skin area to be imaged, subjects were located in a rest position: sitting or supine. Subjects were asked to stay still and breath normally during the measurements. Measurements started with capturing an RGB frame. During the RGB imaging, the LEDs were switched on and the laser illumination was blocked via the motorized shutter. After the RGB imaging, the LEDs were turned off while the laser illumination was allowed on the subject and the monochrome camera acquired speckle frames for 7 seconds. All of the experiments were performed by the same operator (Mirjam J. Schaap), in the same room, with the curtains closed and room illumination switched off. The operator was instructed to perform the handheld measurements while conveniently positioned, the arm bent at almost 90 degrees and keeping the handheld probe normally (i.e. without over-concentration). For the mounted measurements, the handheld LSCI device was placed on a tripod.

\subsubsection{Data analysis}

Acquisition and processing codes in the form of user-interfaces were programmed in MATLAB R2019b. The seven-step perfusion imaging procedure is summarized in Fig. 5.2. All the steps are explained in detail in the rest of this section.

\section{Step 1: Marker segmentation}

A stack of raw speckle frames of a representative handheld measurement including movements of the handheld LSCI system and the patient is shown in Visualization 5.1. The frames were segmented with the aim to prepare them for tracking and alignment. A region of interest (ROI) was manually selected in the first frame that included boundary-indicating marked points, which was only used for the purpose of marker segmentation and frame alignment (see Fig. 5.3(a-b)). Then, a normalized and inverted version of the cropped frame was made with a cut-off value of 25 out of 255 


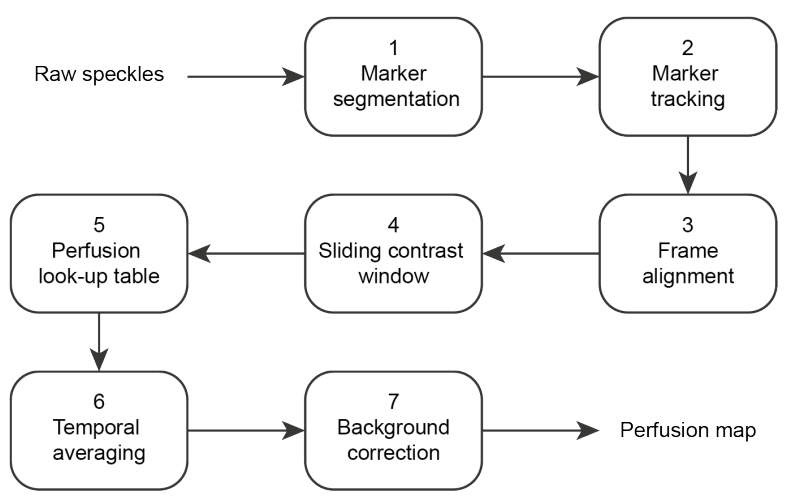

Fig. 5.2: Analysis workflow for perfusion imaging. 1) Choosing an area within the speckle frames that includes markers and run the segmentation algorithm [25, 26]. 2) Localization of segmented markers for the entire stack of frames and calculation of horizontal and vertical on-surface speed elements. 3) Translating the frames based on the computed two dimensional displacements with respect to the reference frame. 4) Applying a sliding window for local calculation of the speckle contrasts and formation of a contrast map per raw speckle frame. 5) Converting contrast maps to perfusion maps based on a pre-saved lookup table. 6) Formation of a denoised perfusion map by temporal averaging of the whole stacks of perfusion maps. 7) Making a background corrected perfusion map with normalization of the input perfusion map by the background perfusion value.

such that the markers had a higher intensity than the surrounding tissue (Fig. 5.3(c)). This image was thresholded at 0.8 to provide the segmentation (Fig. 5.3(d)). The background speckle pattern differs for each frame and therefore should be removed from all frames. To do so, we applied the MATLAB software package 'localized active contour' [25, 26], which implements image segmentation in mean separation (MS) mode [27, 28] shown in Fig. 5.3(e) with the parameters listed in Table 5.1. A Gaussian sliding window of standard deviation 3 was applied to the segmented image (Fig. 5.3(f)). Finally, the product of the cropped version shown in Fig. 5.3(b) with the Gaussian filtered segmented version shown in Fig. 5.3(f) was used for tracking and alignment.

Table 5.1: Parameter setting for the localized active contour segmentation employed in mean separation (MS) mode.

\begin{tabular}{llr} 
Symbol & Parameter & Value \\
\hline$N$ & Number of iterations & 20 \\
$r a d$ & Side length of the square window & 1 \\
$\alpha$ & Coefficient to balance the image fidelity and regularization terms & 0.09 \\
$\varepsilon$ & Value for Delta and Heaviside step functions & 1
\end{tabular}




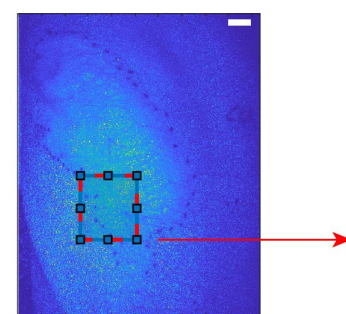

(a)

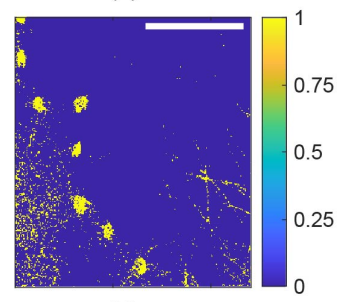

(d)

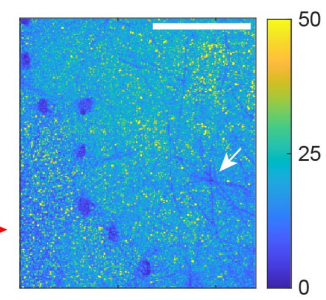

(b)

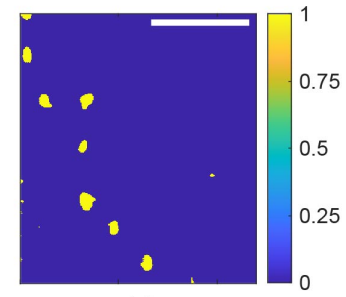

(e)

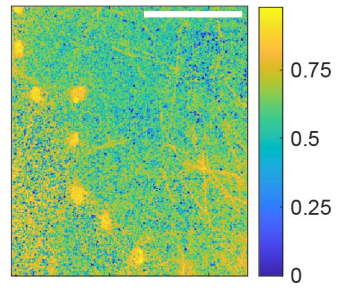

(c)

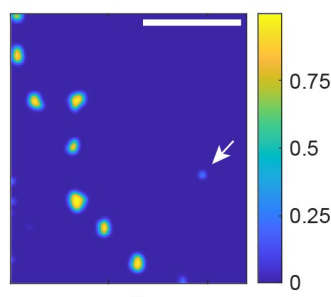

(f)

Fig. 5.3: Marker segmentation in a speckle intensity frame. (a) Full frame speckle pattern including boundary markers. Scale bars, $10 \mathrm{~mm}$. (b) Cropped area shown in (a) with a maximum value of 255 . (c) Normalized and inverted version of (b). (d) Thresholded version of (c) including a speckle pattern. (e) Mean separation segmentation. (f) Gaussian filtering. White arrows indicate a natural landmark formed by intersection of hairs.

\section{Steps 2-3: Marker tracking and frame alignment}

After marker segmentation of all frames, the first segmented frame of each experiment was used as the reference frame. Displacements of the rest of the frames with respect to the reference frame were detected by IAT MATLAB toolbox [29] that works based on maximization of enhanced correlation coefficients [30]. Here, the inputs are: the number of iterations $N=20$; the number of levels for multi-resolution execution 2; the type of geometric transformation 'translation'; and the initial transformation per frame is the translation elements of the previous frame. This way, a concurrent localization is obtained. The output is a matrix called 'warp' that contains horizontal and vertical displacement elements (see Visualization 5.2). The warp matrix was then used for two purposes; (1) computation of on-surface speeds $\left(v_{x}, v_{y}\right)$ by time derivation of the horizontal and vertical position elements $(x, y)$ and (2) full frame alignment of the speckle raw frames by translating each frame based on the corresponding warp matrix elements in the opposite direction (see Visualization 5.3).

The root-mean-square-error (RMSE) distance for each measurement is calculated as

$$
d_{\mathrm{RMSE}}=\sqrt{\frac{1}{T_{m}} \int_{0}^{T_{m}}(x(t)-\bar{x})^{2}+(y(t)-\bar{y})^{2} d t},
$$


where $T_{m}$ is the measurement time (approximately 7 seconds) and $(\bar{x}, \bar{y})$ is the mean location for each experiment. The average on-surface speed per measurement is calculated as

$$
\bar{v}=\frac{1}{T_{m}} \int_{0}^{T_{m}} \sqrt{v_{x}^{2}(t)+v_{y}^{2}(t)} d t .
$$

For the mounted experiments, since the movements were rather small, the tracking could be done for the whole stacks of raw speckle frames. However, due to the sudden displacements of the probe during some handheld experiments, the tracking algorithm became unstable and therefore only part of those experiments could be aligned and considered for motion and perfusion analysis. A summary of the percentages of aligned frames can be found in Supplementary Tables 5.2-5.12.

\section{Step 4: Speckle contrast and sliding window}

The expression for speckle contrast is[31]

$$
C=\frac{\sigma_{I}}{\bar{I}}
$$

where $\sigma_{I}$ and $\bar{I}$ are the standard deviation and mean values of intensity fluctuations respectively, observed by a camera. To convert the speckle raw frames into the socalled contrast maps, a window spatially sweeps over every speckle frame individually in order to compute the local contrast values according to Eq. (5.3). In order to implement the algorithm with an efficient processing time, we have used the sliding convolution technique[32] with a window size of $9 \times 9$ pixels $\left(0.8 \times 0.8 \mathrm{~mm}^{2}\right)$. Visualization 5.4 illustrates the stack of contrast maps calculated after alignment of raw speckle frames. Based on the definition of fully dynamic speckle patterns [31] and time integrated dynamic speckles only the positive contrast values below unity are valid for consideration. For this reason, the values exceeding unity in speckle contrast are clipped. These are mainly the regions outside the imaged skin area where little light is received by the camera sensor. This results in low mean intensity and therefore the contrast raises above 1 (see Eq. (5.3)).

\section{Step 5: Perfusion estimation model}

With random motion of the scatterers and a negative exponential approximation for the auto-correlation function of a field, time integrated dynamic speckle patterns have the contrast[11]

$$
C=\sqrt{\frac{\tau_{c}}{2 T}\left(1-e^{-\frac{2 T}{\tau_{c}}}\right)}
$$


where $\tau_{c}$ and $T$ represent correlation and camera integration times, respectively. The correlation time is defined as the time it takes for speckle field auto-correlation to reach $1 / e$ of its maximum value. Here, the line-of-sight velocity distribution is approximated to be Lorentzian (i.e. a function exponentially decaying with time and reaches $1 / e$ at time $\tau_{c}$ ) and single scattering of the detected light is taken into account. The simplest characteristic velocity is defined as [33]

$$
v_{c}=\frac{\lambda}{2 \pi \tau_{c}}
$$

where $\lambda$ is the wavelength of light. By definition, $v_{c}$ is of dimension distance per time. Note that the focus of this work is not to quantify the blood flow or volumetric flux but to study the relative changes of $v_{c}$ as a function of the observed speckle contrast.

In order to estimate a perfusion value $\left(P_{\text {est. }}\right)$ by measuring a speckle contrast $\left(C_{\text {meas. }}\right)$, a lookup table was made as following. An array $v_{c}$ was formed on the interval $\left[0,10^{-3}\right]$. Then, for each element of the array $v_{c}$ a speckle contrast $(C)$ was calculated based on Eqs. (5.4-5.5) with $\lambda=671 \mathrm{~nm}$ and $T=10 \mathrm{~ms}$. Since a typical contrast value measured on the calibration suspension is $C_{\text {ref. }}=0.2$, the array $v_{c}$ was scaled to show a reference (and arbitrary) perfusion $p_{\text {ref. }}=250$ at $C_{\text {ref. }}$. Therefore, the calibrated lookup table was a plot of $C$ versus $P=\frac{v_{c}\left(T, C_{\text {ref. }}\right)}{p_{\text {ref. }}} v_{c} \times 10^{6}$ (see Fig. 5.5(b)). The quantity $P$ is referred to as perfusion (a.u.) throughout this work. Using this lookup table, stacks of speckle contrast maps were converted to perfusion maps by pixel-based linear interpolation. The reason of creating a lookup table is that $v_{c}$ cannot be written as a closed-form function of $C$.

\section{Step 6: Temporal averaging}

The perfusion maps for each experiment were temporally averaged to make a smoothed perfusion map of reduced speckle noise[34]. Since the temporal resolution is not of interest in this work, averaging of successive frames is a straightforward method of making a representative perfusion map, also with lower influence of heart pulsatility.

\section{Step 7: Background correction}

As a measure of the ratio between perfusion in skin lesions to that of unaffected skin (background perfusion), a background correction step has been introduced. To do so, three ROIs on the healthy skin around a lesion are manually selected and the average perfusion of the selected areas, i.e. background perfusion $\overline{p_{b}}$, is calculated. Then, the whole temporally averaged perfusion map is divided by $\overline{p_{b}}$ in order to form the background corrected perfusion map [7] where the background perfusion is approximately 1 (Supplementary Tables 5.2-5.12). 


\subsubsection{Processing times}

The processing times can be categorized in measurement time and post processing time. The measurements were carried out with a processor Intel(R) Core(TM) i7 $3770 \mathrm{CPU} @ 3.4 \mathrm{GHz}$ and RAM capacity of $16 \mathrm{~GB}$. The total time for a single measurement and saving was $49 \pm 1$ seconds. The program shows perfusion maps (with a sliding window size of $21 \times 21 \mathrm{px}$ on a frames size of $1536 \times 2048 \mathrm{px}$ ) in a preview mode with a rate of $1.46 \pm 0.02 \mathrm{~Hz}$.

The post-processing of the measurements was carried out on a desktop computer with a processor Intel(R) Core $(\mathrm{TM}) \mathrm{i} 7-8700 \mathrm{~K}$ CPU @ 3.70GHz with a RAM capacity of $16 \mathrm{~GB}$. A sample segmentation and alignment in a region of $175 \times 240 \mathrm{px}$ took 129 seconds. The temporal averaging of all 211 frames of a measurement took 109 seconds. The background correction took 45 seconds. Note that the post processing time not only depends on the computer performance, but also it depends on the user skills in loading files and choosing regions in an image. Therefore, the entire post-processing of a measurement can be performed in 5 minutes.

\subsubsection{Statistical analysis}

The perfusion of handheld and mounted measurements was analyzed using descriptive statistics. In addition, paired t-tests were performed between all temporally averaged mounted-handheld measurement pairs. This was done for the mean lesion perfusion in measurements pairs both before (temporally averaged values) and after background correction. Also, the intraclass correlation coefficients (ICCs), a measure for the amount of agreement with a value of 1 reflecting perfect agreement, were calculated for the aforementioned measurement pairs. Statistical analyses were performed with Statistical package SPSS, version 25 (IBM). A two-sided $p<0.05$ was considered statistically significant.

\subsubsection{In-vitro and in-vivo evaluation of the perfusion estimation model in a healthy volunteer}

For the in-vitro experiment, the handheld LSCI system was mounted on a translational stage facing perpendicular to a Delrin plate and a linear speed range of $V=[0,10] \mathrm{mm} / \mathrm{s}$ during 10.35 seconds was applied. an example of this experiment is shown in Visualization 5.5 for an exposure time of $T=10 \mathrm{~ms}$.

Similarly, for the in-vivo experiments, that were performed before usage in psoriasis patients, the LSCI system was mounted on a translational stage to which a linear speed range of $V=[0,10] \mathrm{mm} / \mathrm{s}$ during 3.3 seconds was applied. The study consisted of two phases. In the first phase, the skin had a normal perfusion level. In the second phase, the measurement was carried out 15 minutes after applying $0.2 \mathrm{ml}$ vasodilating 


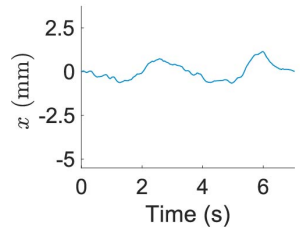

(a)

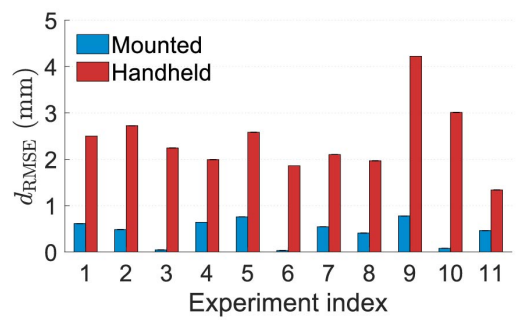

(e)

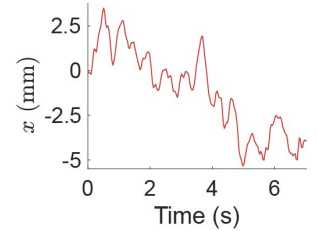

(c)

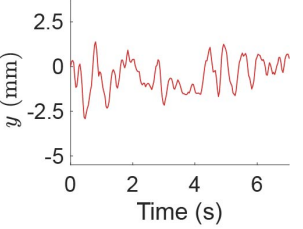

(d)

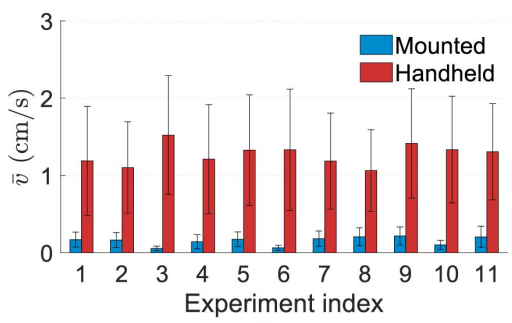

(f)

Fig. 5.4: Motion detection with ECC technique and statistical comparison of calculated on-surface speeds for mounted and handheld measurements. Positions of the beam along (a) horizontal and (b) vertical axes in a mounted experiment. Positive direction in horizontal displacements $(x)$ corresponds to right-side orientation in real world coordination. Positive direction in vertical displacements $(y)$ corresponds to downside orientation in real world coordination. Positions of the beam along (c) horizontal and (d) vertical axes in a handheld experiment. In (a-d) data of experiment index 1 is shown. Overview of the (e) root-mean-square-error distance from average location of each experiment and (f) on-surface speeds (mean \pm standard deviation) for the entire time trace of each experiment.

cream (60 gr Midalgan cream Extra Warm, Qualiphar, Meppel, The Netherlands) on an area of $20 \times 5 \mathrm{~cm}^{2}$. The second phase is referred to as 'high perfusion level'.

For both the in-vitro and in-vivo experiments, a $20^{\circ}$ top hat engineered diffuser (Thorlabs ED1-S20-MD) with square scattered shape was used to expand the laser beam. The speckle frames were captured with an exposure time of $T=25 \mathrm{~ms}$, a frame rate of $40 \mathrm{~Hz}$ and a gain of $0 \mathrm{~dB}$. The speckle contrast for each captured frame was calculated from a windows of size $150 \times 150$ pixels.

\subsection{Results}

\subsubsection{Magnitude of movements}

Relative displacements of the camera and targeted markers around the lesions were observed in all experiments. In mounted experiments displacements are caused by patient movements and in handheld experiments displacements are caused by a combination of the probe and the patients movements. Localization and on-surface speed calculation for the handheld measurement of experiment index 1 has been 


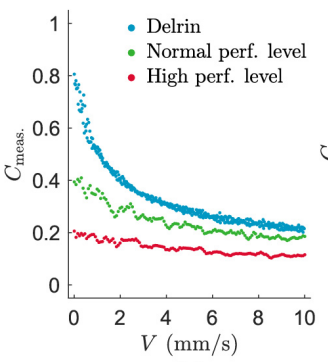

(a)

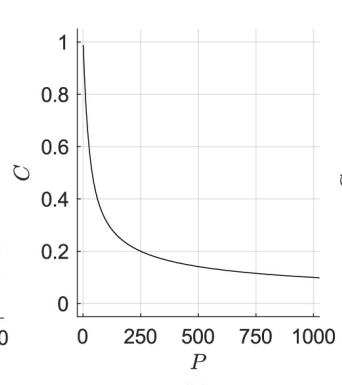

(b)

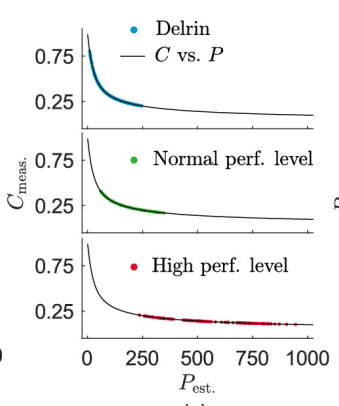

(c)

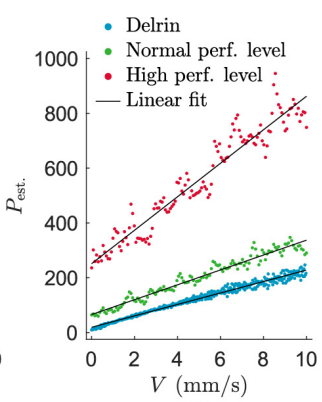

(d)

Fig. 5.5: Model examination with motorized displacements of the LSCI system for phantom and in-vivo subjects. (a) Measured speckle contrast versus the applied speed. perf.: perfusion. (b) Contrast-perfusion lookup table. (c) Linear interpolation of the estimated perfusion data points from the measured speckle contrast according to the contrast-perfusion lookup table. vs.: versus. (d) Estimated perfusion as a function of the applied speed overlapped with a linear fit for each experiment.

demonstrated in Visualization 5.2. Figure 5.4 (a-b) illustrates horizontal and vertical positions with respect to the first frame for a representative mounted experiment. The RMSE-distance and the average on-surface speed values for this experiment are $0.6 \mathrm{~mm}$ and $1.7 \mathrm{~mm} / \mathrm{s}$, respectively. The corresponding handheld experiment has been depicted in Fig 5.4 (c-d) with an RMSE-distance and average on-surface speed values of $2.5 \mathrm{~mm}$ and $11.9 \mathrm{~mm} / \mathrm{s}$, respectively.

Figure 5.4(e) gives an overview of comparison between the RMSE-distance with an average of $0.4 \mathrm{~mm}$ and $2.4 \mathrm{~mm}$ for all of the mounted and handheld measurements, respectively. In Fig. 5.4(f), the on-surface speeds are depicted which are calculated based on Eq. 5.2. The averages of the absolute on-surface speed values (Eq. 5.2) shown in Fig. 5.4(f) are $1.5 \mathrm{~mm} / \mathrm{s}$ and $12.7 \mathrm{~mm} / \mathrm{s}$ for the mounted and handheld measurement pairs, respectively, which shows an increase with a factor of 8.5.

\subsubsection{Contrast-perfusion model examination}

The purpose of the model examination is to explore the estimated perfusion response to a linear increase of the applied speed using the contrast-perfusion lookup table described in 5.2.4. Figure 5.5(a) illustrates the measured speckle contrast versus the applied speed observed on a static scattering medium (Delrin) and on a forearm of a healthy subject with both normal and high perfusion levels. The following can be observed. (1) The speckle contrast at zero-speed is not unity for Delrin as expected theoretically since Delrin is a static object; (2) The measured speckle contrast decays non-linearly as a function of the applied speed for all three cases; (3) For the same applied speed profile, the dynamic range of the speckle contrast decreases as the subject includes more internal movements. 


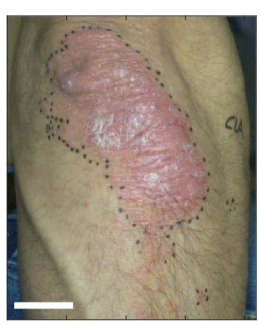

(a)

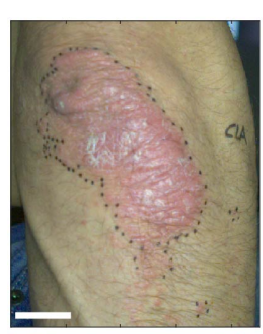

(b)

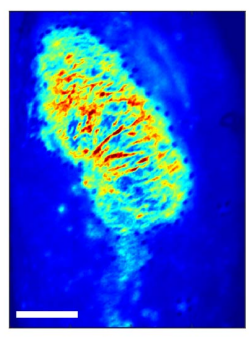

(c)

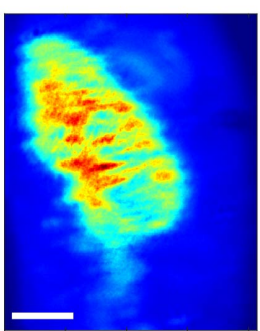

(d)

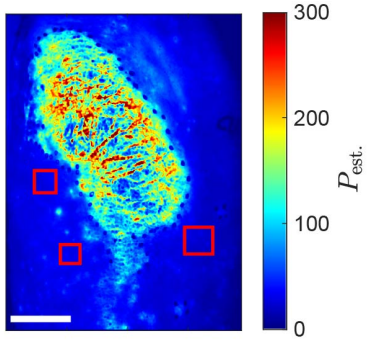

(e)

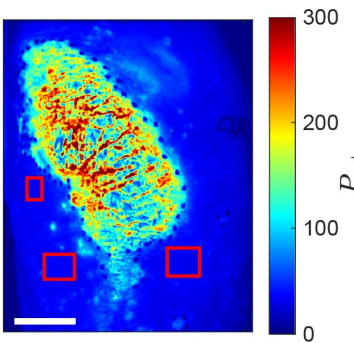

(f)

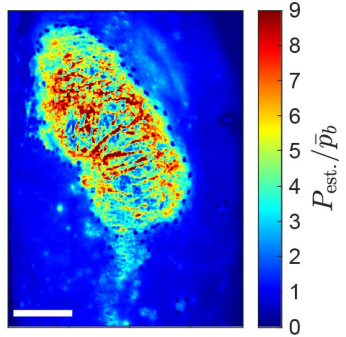

(g)

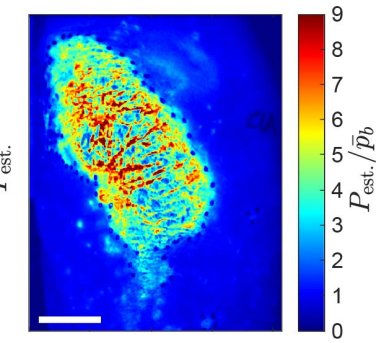

(h)

Fig. 5.6: Visual comparison of a representative mounted (top row) and the corresponding handheld (bottom row) experiments. (a-b) RGB photographs. Scale bars, $25 \mathrm{~mm}$. Temporally averaged perfusion maps (c-d) before and (e-f) after the alignment. Red rectangles indicate areas in which background perfusion values $\left(\bar{p}_{b}\right)$ are calculated. $P_{\text {est. }}$ : estimated perfusion. $(\mathrm{g}-\mathrm{h})$ Background corrected perfusion maps. Data of experiment index 1 is shown.

The predefined contrast-perfusion curve is shown in Fig. 5.5(b) which was introduced in Eqs. (5.4-5.5). Using this curve a perfusion value is assigned to each measured speckle contrast data point. The set of estimated perfusion data points for the three experiments is depicted in Fig. 5.5(c) that gives us a visual impression to which part of the curve these data points belong. Finally, a plot of estimated perfusion versus the applied speed is shown in Fig. 5.5(d) where a rather linear relation for each case is observed. This relation is investigated by a fit function as;

$$
P_{\text {est. }}=a V+b \text {. }
$$

The pair of $(a, b)$ for Delrin, low and high perfusion levels are obtained as $(20.9,19.1)$, $(27.1,65.7)$ and $(61.1,251.4)$, respectively. The $R^{2}$ values for Delrin, low and high perfusion levels are $0.98,0.95$ and 0.92 , respectively. The $y$-intercept ( $b$ value) of this fit function points at two issues: (1) experimental imperfection (i.e. the fact that $b$ is non-zero for Delrin) which can be due to small vibration of the setup, camera noise, and laser source; and (2) baseline perfusion (i.e. perfusion level without movement artefact). Also, both the slopes and $y$-intercepts increase as the internal movements of the subjects increase. Note that the fluctuations in high perfusion level shown in Fig. 5.5(d) are noticeably driven by the heart pulsations. 


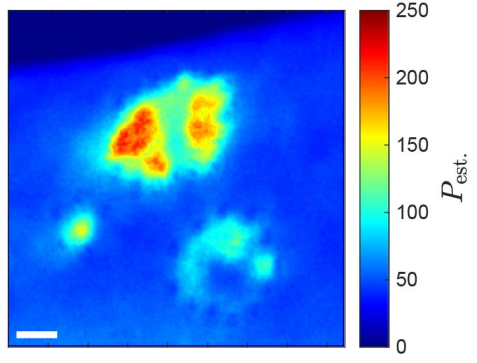

(a)

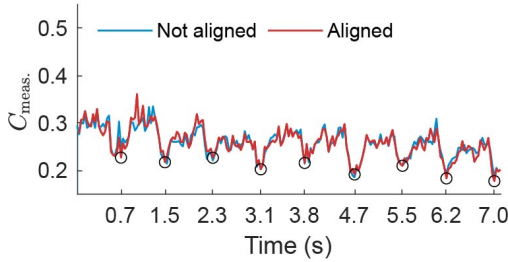

(c)

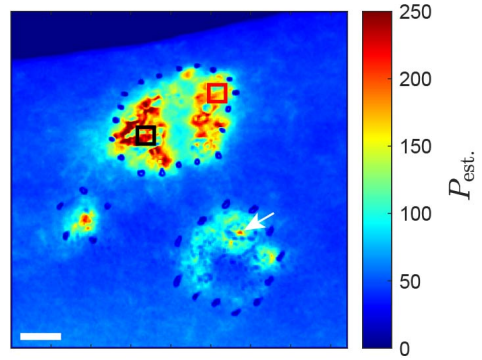

(b)

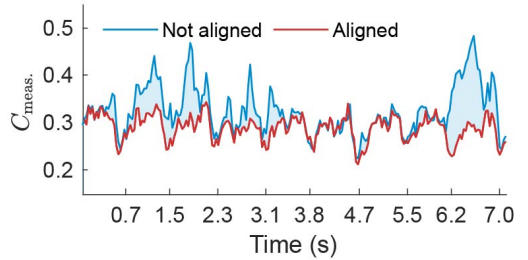

(d)

Fig. 5.7: Spatiotemporal analysis of speckle frames alignment. Data of experiment index 6 is shown. (a) Temporally averaged perfusion map of a representative handheld measurement without alignment. $P_{\text {est. }}$ :estimated perfusion. Scale bars, $10 \mathrm{~mm}$. (b) Temporally averaged perfusion map of the same measurement after alignment. White arrow indicates a small region with a rather higher perfusion value which is not distinguishable without alignment. (c) Temporal fluctuation of the measured speckle contrast $\left(C_{\text {meas. }}\right)$ during the corresponding handheld measurement with and without alignment. The region of interest for the calculation is shown as the black square in (b). Open circles: manually selected sudden drops in the speckle contrasts possibly driven by the heartbeats. (d) Measured speckle contrast versus time during the handheld measurement where the calculation region is shown as the red square in (b).

\subsubsection{RGB imaging and visual comparison of perfusion maps}

The RGB image of a mounted measurement is shown in Fig. 5.6(a) where the clinically visible lesion and boundary-indicating black marking points are apparent. The temporally averaged perfusion maps of this measurement before and after the alignment are illustrated in Fig. 5.6(c) and (e), respectively. Although there is no movement of the handheld LSCI system during this mounted measurement, patient movement causes slight blurring of this perfusion map before the alignment. The healthy skin around the psoriasis lesion has a lower perfusion in comparison to the lesion. The low intensities of the black markers make the speckle contrasts exceed unity; thus, the corresponding perfusion values become close to zero. The associated background corrected perfusion map is depicted in Fig. 5.6(g) with a background perfusion level of approximately 1 . The corresponding RGB image, temporally averaged and background corrected perfusion maps for the handheld experiment are demonstrated in Fig. 5.6(b), (d), (f), and (h), respectively. For an overview of the 
entire collection of measurement pairs, see Supplementary Figs. 5.9-5.19.

\subsubsection{Influence of alignment on spatiotemporal perfusion profiles}

A temporally averaged handheld perfusion map without alignment is shown in Fig. 5.7(a). This perfusion map is blurred due to the movements. The aligned perfusion map is shown in Fig. 5.7(b), in which the marking points look sharper. Two regions are selected to examine the temporal fluctuation of the speckle contrast. The first ROI (the black square shown in Fig. 5.7(b)) is placed far from the lesion boundaries. The contrast of this region in the time domain with and without alignment is shown in Fig. 5.7(c), where the graphs are to some extent similar with a root mean square error (RMSE) of 0.02. The dips in the speckle contrast pattern are a suggestive heartbeat signal with an estimated rate of 77 beats/minutes. A second ROI was selected in a region close to the lesion boundary (the red square shown in Fig. 5.7(b)) and the speckle contrast time traces were computed (See Fig. 5.7(d)). For a demonstration of the analysis without and with tracking, see Visualization 5.6 and Visualization 5.7, respectively. This time, at the interval when the ROI overlaps the markers or goes beyond the lesion area, the calculation of speckle contrast is affected and could be unreliable when the region is not tracked. The RMSE for this pair is 0.06 which is, in this example, 3 times of that on the first ROI. It is worth noting that the suggestive heartbeat pattern could not be detected in all measurements even in mounted mode.

\subsubsection{Accounting for movement artefacts and effect of background cor- rection}

A comparison between temporally averaged and background corrected perfusion maps gives a measure of similarity between the pairs of mounted and handheld measurements. Fig. 5.8(a-b) summarizes the mean perfusion values of the entire lesion indicated by black marking points on the skin for all measurement pairs (Supplementary Figs. 5.9-5.19). It is worth noting that the collections $\{1,4,8\},\{3,6,10\},\{5,9\}$, $\{7,11\}$ and $\{2\}$ belong to same patients captured in different days, i.e. data of 5 patients are illustrated.

In all experiments, the temporally averaged handheld measurements (Fig. 5.8(a)) showed greater mean perfusions. The temporally averaged perfusions for mounted and handheld measurements are $94.2 \pm 29.5$ (mean \pm std, $n=11)$ and $117.4 \pm 40$ (mean \pm std, $n=11$ ) with medians of 82.3 and 101.6, respectively. An absolute difference of $26.2 \pm 28.5 \%$ (mean \pm std, $n=11$ ), and an absolute median difference of $23.4 \%$ exist between the mounted and handheld temporally averaged perfusion map pairs due to the movement artefacts. A paired t-test revealed that the difference between the handheld and mounted pairs of temporally averaged perfusion maps is statistically significant $(p=0.02)$. 


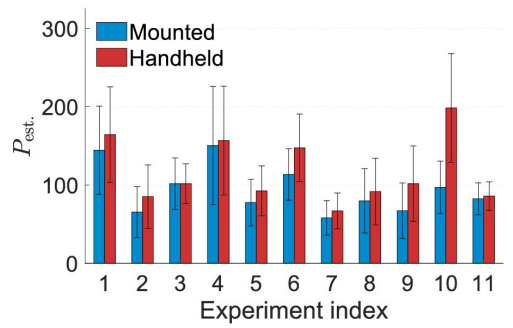

(a)

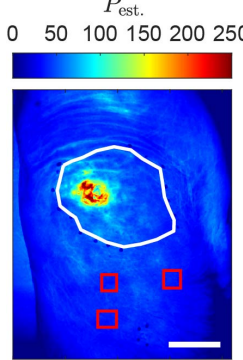

(c)

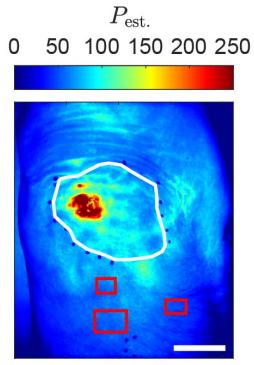

(d)

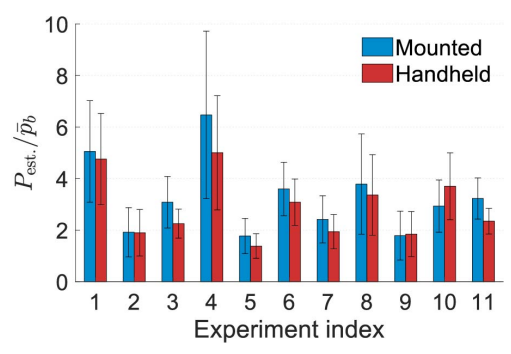

(b)

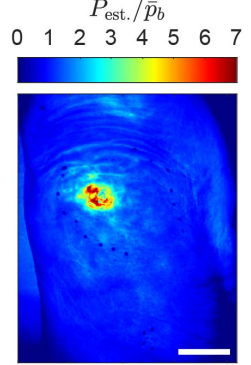

(e)

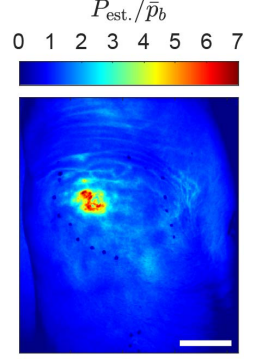

(f)

Fig. 5.8: Visualization and statistical comparison between temporally averaged and background corrected perfusion maps per pairs of mounted and handheld experiments. Overview of average perfusion over the lesion areas for (a) temporally averaged and (b) background corrected perfusion maps. $P_{\text {est. }}$ : estimated perfusion; $\bar{P}_{b}$ : background perfusion. Data are reported as mean \pm standard deviation. Representative temporally averaged perfusion maps of (c) mounted and (d) handheld measurements of experiment index 9. Red rectangles: manually selected regions in which background perfusion values $\left(\bar{P}_{b}\right)$ are calculated. White polygons: manually selected lesion areas. Background corrected perfusion maps of corresponding (e) mounted and (f) handheld measurements. Scale bars, $25 \mathrm{~mm}$.

The mean perfusions of the corresponding lesions in the background corrected perfusion maps are shown in Fig. 5.8(b). The regions chosen for background correction are provided in Supplementary Figs. 5.9-5.19. The background corrected perfusions for mounted and handheld cases are $3.3 \pm 1.4$ (mean \pm std, $n=11)$ and $2.9 \pm 1.2$ (mean \pm std, $n=11$ ) with medians of 3.1 and 2.3, respectively. After the background correction, the absolute difference between the mounted and handheld pairs becomes $16.4 \pm 9.3 \%$ (mean \pm std, $n=11$ ), with an absolute median difference of $23.8 \%$. A paired t-test revealed that the difference between the handheld and mounted measurement pairs after the background correction remains statistically significant $(p=0.04)$.

Therefore, background correction helps to decrease the absolute difference between the mounted and handheld pairs caused by movement artefacts on average from $26.2 \%$ to $16.4 \%$ although such improvement is negligible when median values are considered. These movement-induced difference margins are to be considered when ana- 
lyzing handheld perfusion maps. The paired t-tests revealed that background correction does not guaranty that the mounted-handheld pairs become insignificantly different. However, the ICCs for the aforementioned pairs were 0.603 (95\% CI $0.02-0.877$, corresponding with moderate agreement) and 0.874 (95\% CI $0.514-0.967$, corresponding with good agreement) for the temporally averaged and background corrected pairs, respectively. This suggests that background correction increases the agreement between the mounted-handheld measurement pairs.

In Fig. 5.8(c-f) a visual example is shown in which, having the same color scale, the pair of background corrected images are more comparable to each other. Note that the average perfusion values of the handheld measurements after the background correction shown in Fig. 5.8(b) are, mostly, lower than the mounted measurements. To elaborate on the reason, we argue the following. (1) If in the entire image, the perfusion value is increased with a constant value, the relative increase for the background perfusion (which is low) exceeds the relative increase of the lesion value (which is high). (2) This effect is mitigated since the motion-induced perfusion increment increases with the perfusion (as shown in Fig. 5.5(d), however this effect cannot fully undo the effect of (1)).

\subsection{Discussion}

The performance of handheld LSCI was studied in detail and the outputs were compared with mounted experiments in psoriasis patients using the HAPI prototype (Fig. 5.1). We introduced a post-processing procedure of handheld LSCI for step-by-step analysis of acquired raw speckle frames to reach a representative perfusion map which matches the mounted perfusion map (Fig. 5.2). The advantage of frame alignment in LSCI was previously shown by Richards et al. [35] intraoperatively and in a mounted modality. In this study, we employed segmentation and image alignment for LSCI on psoriasis lesions in a handheld modality. The size of a selected ROI for aligning handheld measurement frames should not be too large as an account for the computation time, and not too small, as an account for having stable results.

For tracking and image alignment, the geometric transformation is chosen as 'translation' which means that the rotations are ignored. As a consequence, the aligned sequence of speckle frames include residual movements in the form of rotations which is apparent in Visualization 5.3 although the temporally averaged perfusion maps are sharp enough to neglect the rotations. Despite the ability of the employed ECC image alignment method to account for rotational transformation as well as translational, the rotational transformation cannot be done since the center point for an ROI is not necessarily the same as the center point of the whole frame. Although several state-of-the-art methods for segmentation and alignment exist, the combination of MS segmentation and ECC image alignment for this work has been chosen since they are 
robust, computationally cheap and can effectively remove the speckle patterns in the background. Here a proof-of-concept for alignment and motion detection within the speckle intensity frames is shown, that highlights the potential of this method for other handheld applications or those including patient movements in the field.

The existence of (natural or artificial) markers on the skin is a key requirement for optimal perfusion imaging and image alignment. In this study, black marking points were applied. Naturally, for the applicability in daily clinical practice, the use of natural (skin) landmarks would be preferable. Figure 5.3(f) shows an example of the detection of a crossing hair region as a natural landmark. However, the possibility and accuracy of tracking natural landmarks with the current algorithm remains a challenge. Alternative solutions such as employing optical-flow algorithm to track the natural landmarks on RGB frames acquired parallel to the speckle frames might be of help.

Relative movements between the handheld probe and subjects are quantified based on the RMSE-distance analysis (Fig. 5.4(e)). The RMSE-distance is a measure of displacements of the acquired frames from the average point (center of gravity). Since the RMSE-distances for both handheld and mounted modes are non-zero, the frame alignment is a required step in order to prevent blurring of the temporally averaged perfusion maps as well as obtaining a reliable temporal perfusion profile when choosing an area on a perfusion map. The average absolute on-surface speed for handheld measurements is calculated as 8.5 times greater than the mounted ones (Fig. 5.4(f)). In a previous study, the average on-surface speed for handheld measurements by 10 healthy operators on a static object was calculated as $9 \mathrm{~mm} / \mathrm{s}$ where the measurement distance was $20 \mathrm{~cm}$ [18]. In this study, the calculated value is $12.7 \mathrm{~mm} / \mathrm{s}$ with the measurement distance of $40 \mathrm{~cm}$. The increase in the calculated value in this study is due to the added movements of the patients during measurements as well as the increased measurement distance.

The exact relationship between speckle contrast and blood flow is open for research and has been addressed in a range of articles[36, 37]. The theoretical model that we have used to estimate the perfusion assumes a Lorentzian velocity distribution. Although a modified version of this model is proposed by Duncan et al.[38], we used it in its native version (Eqs. (5.4-5.5)) and examined it via in-vitro and in-vivo experiments. We observed a linear relation of the estimated perfusion versus the applied speed and the slope of these lines are not necessarily the same for low and high perfusion levels (see Fig. 5.5(d)). These results cannot be generalized to any human subject due to the complexity of the human body and change of perfusion from one subject to another. However, what these results suggest is that the movement-related perfusion can be subtracted from the measured perfusion in both low and high levels provided that a range of on-surface speeds is accurately measured. This method would make the procedure of movement artefact correction independent of attaching an opaque surface on the tissue for measuring the speckle contrast driven by involuntary 
movements. Another source of movement artefacts, in addition to translation, is the tilting of wavefronts relative to the tissue. However, the impact of wavefront tilting on speckle contrast drop was suppressed by using spherical wave illumination [19].

Three ways for presentation of mounted and handheld experiments have been introduced. First, comparing the pairs of temporally averaged perfusion maps with a localized color-map scaling (Supplementary Figs. 5.9-5.19 (a-b)). Here the best spatial similarity is achieved compared to the other two ways mentioned in the following. Second, comparing the pairs of temporally averaged perfusion maps with the same color-map scaling (Fig. 5.8(c-d) and Supplementary Figs. 5.9-5.19 (c-d)). In this case, the influence of movement-related perfusion is apparent. Depending on the magnitude of movements, handheld measurements are of higher average perfusion values. Third, comparison of the background corrected pairs with the same color-map scaling (Fig. 5.8(e-f) and Supplementary Figs. 5.9-5.19 (e-f)). This step helps to know the perfusion level in a lesion relative to the background perfusion. Moreover, in the analysis of this work, all captured frames were used for temporal averaging. As an improvement, one can choose certain frames with the lowest average perfusion in order to decrease the influence of heartbeat and movement artefact in the temporally averaged perfusion maps. However, based on the type of the analysis and the required accuracy, compensation for movement artefacts may still be necessary for some applications.

While our post-processing procedure is effective in obtaining perfusion maps that are less influenced by involuntary movements, artefacts caused by blurring of the speckle patterns within the exposure time are not corrected. For exact correction of the speckle contrast loss due to motion, both on-surface speed and tilt of wavefronts are required as well as knowledge about optical properties of the tissue. Another factor contributing to speckle blurring within the exposure time is moderately high frequency (i.e. around $40 \mathrm{~Hz}$ ) and low amplitude (i.e. about $1 \mathrm{px}$ ) vibrations that are conducted to the patient's skin (e.g. vibrations transferred from the floor to the skin). This factor poses potential limitation in obtaining a movement-artefact-free measurement. Also, power spectral analysis (PSD) of time-variant local perfusion helps to identify and correct for the influence of cardiac and respiratory rhythms. 


\subsection{Conclusion}

We have examined handheld LSCI in a clinical research setting by introducing a post-processing procedure and made a comparison with mounted measurements. Our results show that handheld measurements are reliable in terms of visual similarity compared to mounted measurements. Enabling handheld perfusion imaging would be highly beneficial for implementation in daily clinical practice. The compact and portable HAPI probe realizes a comfortable skin perfusion measurement for both patients and medical staff. The background correction reduced the absolute difference between the mounted and handheld pairs to $16.4 \pm 9.3 \%$ (mean \pm std, $n=11$ ), with an absolute median difference of $23.8 \%$. Although the differences between the mountedhandheld pairs in both temporally averaged and background corrected cases remain statistically significant, the degree of agreement between them increases after the background correction. Depending on the application in a clinical practice, this difference induced by movement artefacts may be acceptable. The results of this study can be used to advance the LSCI technology towards clinical use, especially for correction of movement artefacts in the handheld mode.

\section{Data availability}

The datasets generated during and/or analyzed during this study to reproduce the graphs within the manuscript (Figs. 2-8) are available in the Figshare repository, https://doi.org/10.6084/m9.figshare.14995083. The temporally averaged and background corrected perfusion maps for experiment indices 2-5, 7-8 and 10-11 are available from the corresponding author on reasonable request. The raw speckle frames for experiment indices 2-11 are available from the corresponding author on reasonable request.

\section{Code availability}

The codes used for analysis of the raw data as user-interface during the current study are available in Supplementary Code S 5.1.

\section{Acknowledgments}

This study was supported by the Open Technology program of the Netherlands Organization for Scientific Research (NWO), Domain Applied and Engineering Sciences, under grant number 14538. We thank Tjitske Bannink and Freek Bielevelt for their valuable help during implementation of the handheld laser speckle contrast imaging system. 


\section{Author contributions}

T.K. designed the handheld perfusion imager. A.C. and T.K. developed the user interface for data acquisition and analysis. A.C. and T.K. conducted the in-vitro phantom experiments. M.J.S. conducted the experiments on psoriasis patients and carried out the statistical analysis. Y.E.B. initialized the MATLAB program for segmentation and alignment. A.C. performed the data analysis and wrote the manuscript. M.M.B.S. and W.S. designed and supervised the study. All authors carefully reviewed and approved the manuscript. A.C. and M.J.S. contributed equally to this work. 


\subsection{Supplementary data}

\section{Experiment index 1}

\begin{tabular}{ccccccc} 
& Time & \% Aligned frames & $\overline{p_{b}}$ & $\bar{I}$ & $C_{\mathrm{s}}$ & $C_{\mathrm{d}}$ \\
\hline Mounted & $9: 34$ & 100 & 28.6 & 15.9 & 0.86 & 0.17 \\
\cline { 2 - 5 } Handheld & $9: 41$ & 100 & 34.5 & & &
\end{tabular}

Table 5.2: Detail information of the measurement pair. $\bar{p}_{b}$ : Average perfusion in the selected background areas. $\bar{I}$ : Mean intensity on Delrin out of 255 . $C_{\mathrm{s}}$ : Speckle contrast on Delrin. $C_{\mathrm{d}}$ : Speckle contrast on scattering suspension.

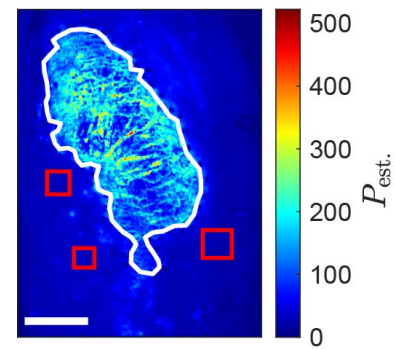

(a)

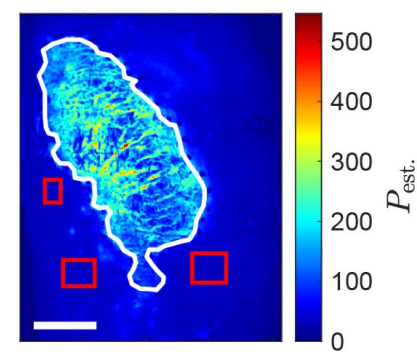

(b)

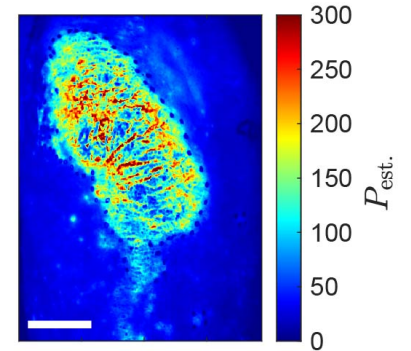

(c)

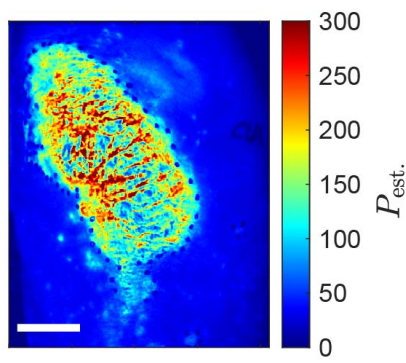

(d)

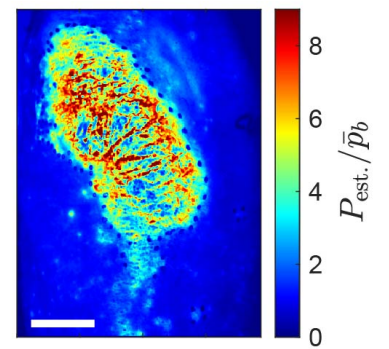

(e)

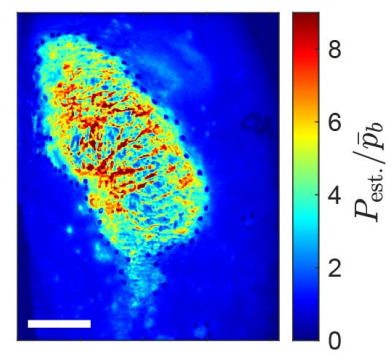

(f)

Fig. 5.9: Comparison of mounted (i.e. (a), (c) and (e)) and handheld (i.e. (b), (d) and (f)) measurements. $P_{\text {est. }}$ : estimated perfusion. Scale bars, $25 \mathrm{~mm}$. Red rectangles: manually selected regions for calculation of background perfusion $\left(\bar{p}_{b}\right)$. White polygons: manually selected lesion areas. Temporally averaged perfusion maps with (a-b) a localized color-map scaling and (c-d) the same color-map scaling. (e-f) Corresponding background corrected perfusion maps with the same color-map scaling. 


\section{Experiment index 2}

\begin{tabular}{|c|c|c|c|c|c|c|}
\hline & Time & $\%$ Aligned frames & $\overline{p_{b}}$ & $\bar{I}$ & $C_{\mathrm{s}}$ & $C_{\mathrm{d}}$ \\
\hline Mounted & $9: 33$ & 100 & 34 & \multirow{2}{*}{13.5} & \multirow{2}{*}{0.8} & \multirow{2}{*}{0.16} \\
\hline Handheld & $9: 35$ & 100 & 44.9 & & & \\
\hline
\end{tabular}

Table 5.3: Detail information of the measurement pair. $\bar{p}_{b}$ : Average perfusion in the selected background areas. $\bar{I}$ : Mean intensity on Delrin out of $255 . C_{\mathrm{s}}$ : Speckle contrast on Delrin. $C_{\mathrm{d}}$ : Speckle contrast on scattering suspension.

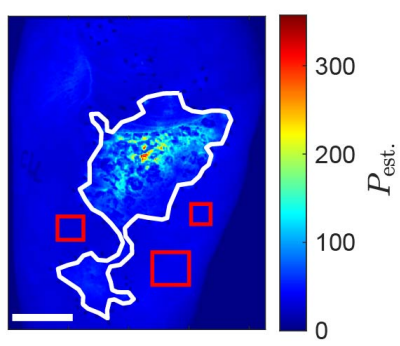

(a)

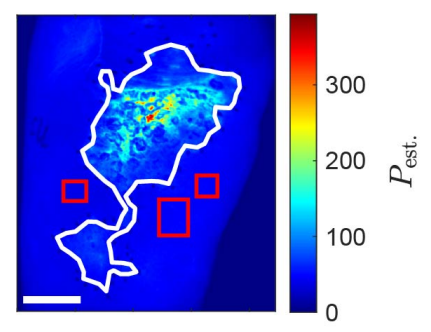

(b)

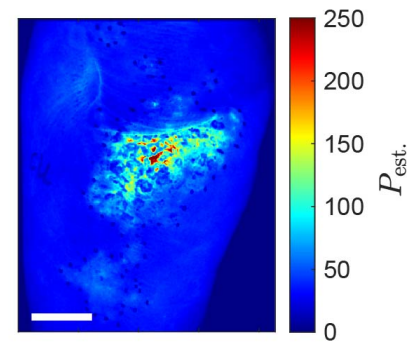

(c)

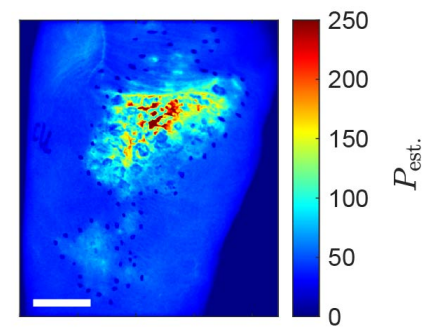

(d)

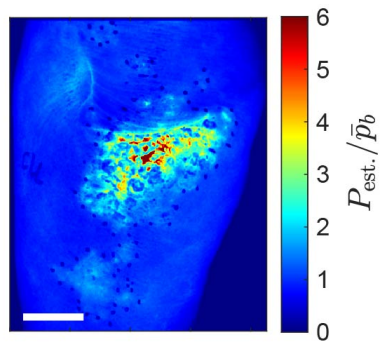

(e)

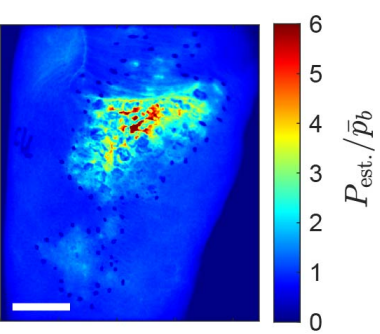

(f)

Fig. 5.10: Comparison of mounted (i.e. (a), (c) and (e)) and handheld (i.e. (b), (d) and (f)) measurements. $P_{\text {est. }}$ : estimated perfusion. Scale bars, $25 \mathrm{~mm}$. Red rectangles: manually selected regions for calculation of background perfusion $\left(\bar{p}_{b}\right)$. White polygons: manually selected lesion areas. Temporally averaged perfusion maps with (a-b) a localized color-map scaling and (c-d) the same color-map scaling. (e-f) Corresponding background corrected perfusion maps with the same color-map scaling. 


\section{Experiment index 3}

\begin{tabular}{|c|c|c|c|c|c|c|}
\hline & Time & $\%$ Aligned frames & $\overline{p_{b}}$ & $\bar{I}$ & $C_{\mathrm{s}}$ & $C_{\mathrm{d}}$ \\
\hline Mounte & $13: 32$ & 100 & 33 & 21 & 00 & (A) \\
\hline Handheld & $13: 41$ & 66 & 45.1 & & & \\
\hline
\end{tabular}

Table 5.4: Detail information of the measurement pair. $\bar{p}_{b}$ : Average perfusion in the selected background areas. $\bar{I}$ : Mean intensity on Delrin out of $255 . C_{\mathrm{S}}$ : Speckle contrast on Delrin. $C_{\mathrm{d}}$ : Speckle contrast on scattering suspension.

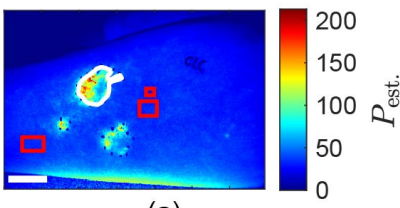

(a)

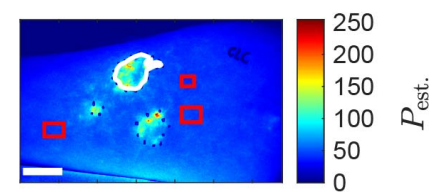

(b)

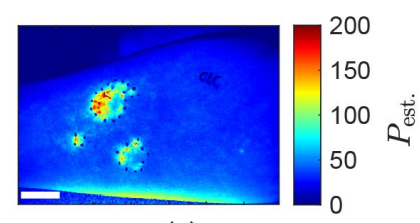

(c)

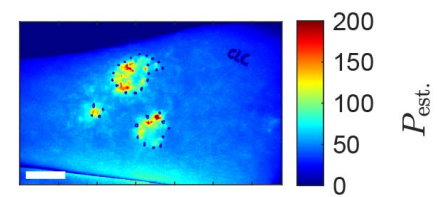

(d)

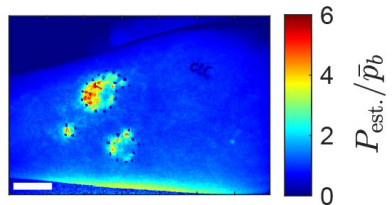

(e)

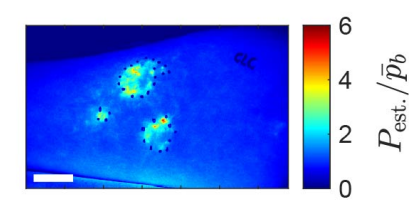

(f)

Fig. 5.11: Comparison of mounted (i.e. (a), (c) and (e)) and handheld (i.e. (b), (d) and (f)) measurements. $P_{\text {est. }}$ : estimated perfusion. Scale bars, $25 \mathrm{~mm}$. Red rectangles: manually selected regions for calculation of background perfusion $\left(\bar{p}_{b}\right)$. White polygons: manually selected lesion areas. Temporally averaged perfusion maps with (a-b) a localized color-map scaling and (c-d) the same color-map scaling. (e-f) Corresponding background corrected perfusion maps with the same color-map scaling. 


\section{Experiment index 4}

\begin{tabular}{ccccccc} 
& Time & \% Aligned frames & $\overline{p_{b}}$ & $\bar{I}$ & $C_{\mathrm{s}}$ & $C_{\mathrm{d}}$ \\
\hline Mounted & $9: 19$ & 100 & 23.2 & & 0.91 & 0.2 \\
\cline { 2 - 6 } Handheld & $9: 16$ & 100 & 31.3 & & &
\end{tabular}

Table 5.5: Detail information of the measurement pair. $\bar{p}_{b}$ : Average perfusion in the selected background areas. $\bar{I}$ : Mean intensity on Delrin out of $255 . C_{\mathrm{s}}$ : Speckle contrast on Delrin. $C_{\mathrm{d}}$ : Speckle contrast on scattering suspension.

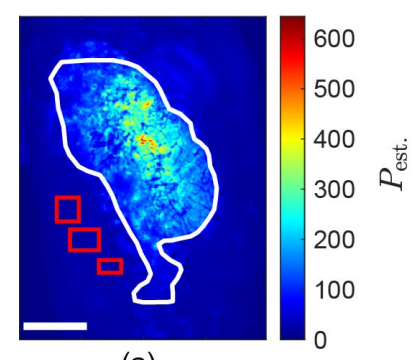

(a)

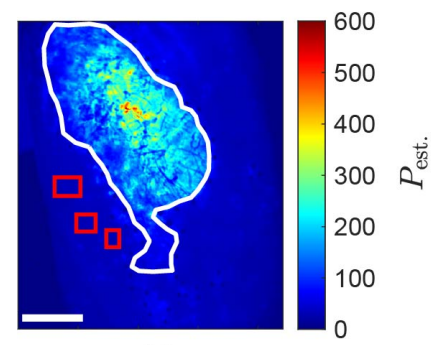

(b)

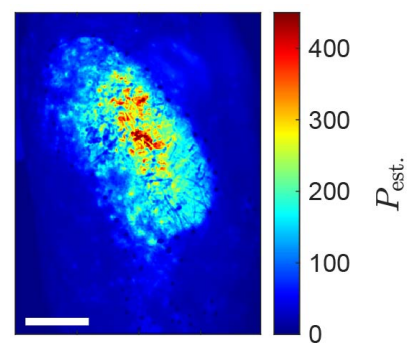

(c)

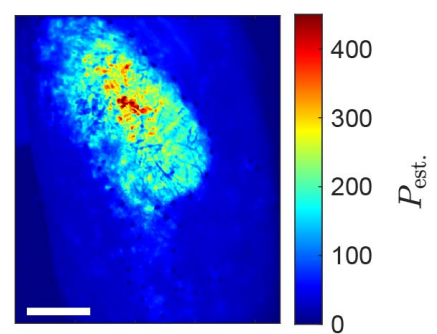

(d)

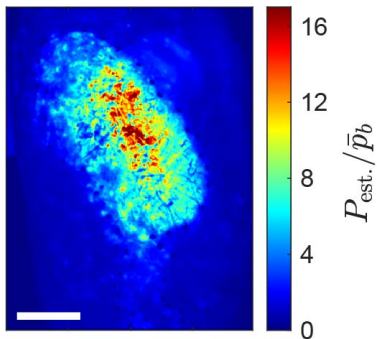

(e)

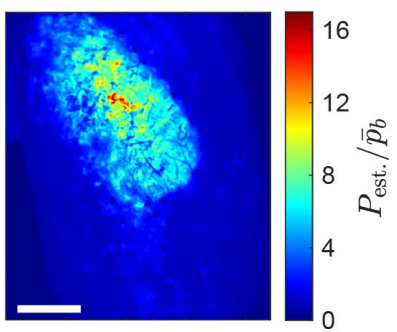

(f)

Fig. 5.12: Comparison of mounted (i.e. (a), (c) and (e)) and handheld (i.e. (b), (d) and (f)) measurements. $P_{\text {est. }}$ : estimated perfusion. Scale bars, $25 \mathrm{~mm}$. Red rectangles: manually selected regions for calculation of background perfusion $\left(\bar{p}_{b}\right)$. White polygons: manually selected lesion areas. Temporally averaged perfusion maps with (a-b) a localized color-map scaling and (c-d) the same color-map scaling. (e-f) Corresponding background corrected perfusion maps with the same color-map scaling. 


\section{Experiment index 5}

\begin{tabular}{ccccccc} 
& Time & $\%$ Aligned frames & $\overline{p_{b}}$ & $\bar{I}$ & $C_{\mathrm{s}}$ & $C_{\mathrm{d}}$ \\
\hline Mounted & $13: 13$ & 100 & 43.8 & & & \\
\cline { 2 - 7 } Handheld & $13: 11$ & 100 & 66.9 & & 0.89 & 0.19
\end{tabular}

Table 5.6: Detail information of the measurement pair. $\bar{p}_{b}$ : Average perfusion in the selected background areas. $\bar{I}$ : Mean intensity on Delrin out of $255 . C_{\mathrm{s}}$ : Speckle contrast on Delrin. $C_{\mathrm{d}}$ : Speckle contrast on scattering suspension.

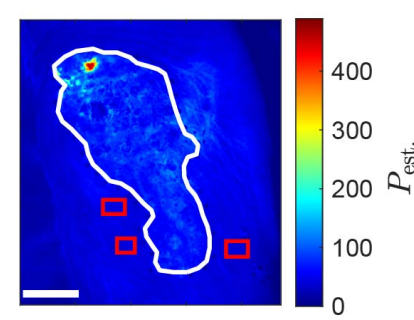

(a)

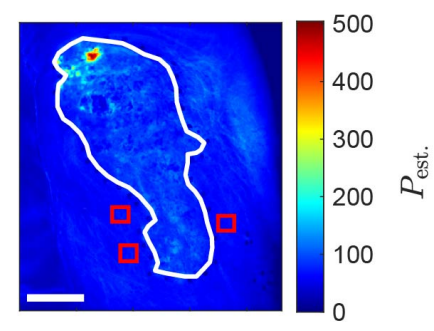

(b)

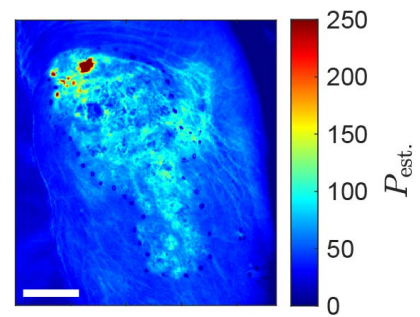

(c)

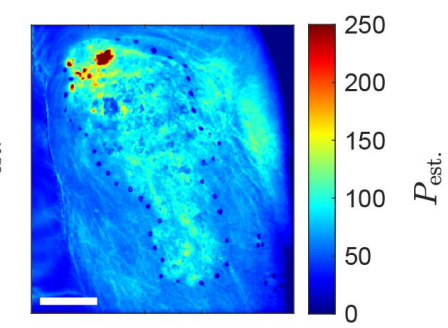

(d)

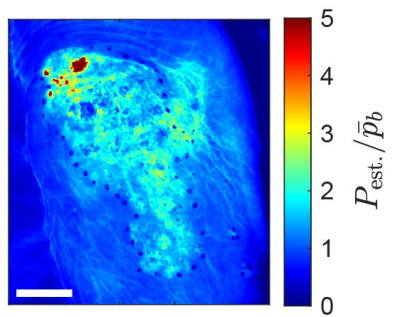

(e)

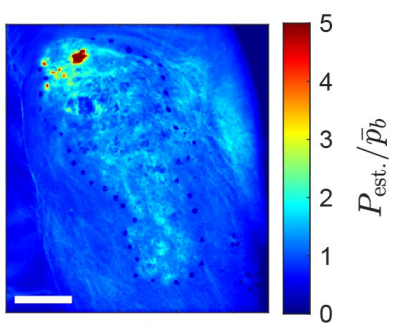

(f)

Fig. 5.13: Comparison of mounted (i.e. (a), (c) and (e)) and handheld (i.e. (b), (d) and (f)) measurements. $P_{\text {est. }}$ : estimated perfusion. Scale bars, $25 \mathrm{~mm}$. Red rectangles: manually selected regions for calculation of background perfusion $\left(\bar{p}_{b}\right)$. White polygons: manually selected lesion areas. Temporally averaged perfusion maps with (a-b) a localized color-map scaling and (c-d) the same color-map scaling. (e-f) Corresponding background corrected perfusion maps with the same color-map scaling. 


\section{Experiment index 6}

\begin{tabular}{ccccccc} 
& Time & \% Aligned frames & $\overline{p_{b}}$ & $\bar{I}$ & $C_{\mathrm{s}}$ & $C_{\mathrm{d}}$ \\
\hline Mounted & $13: 26$ & 100 & 31.5 & & & \\
\cline { 2 - 6 } Handheld & $13: 24$ & 100 & 47.8 & & 0.78 & 0.18
\end{tabular}

Table 5.7: Detail information of the measurement pair. $\bar{p}_{b}$ : Average perfusion in the selected background areas. $\bar{I}$ : Mean intensity on Delrin out of 255 . $C_{\mathrm{s}}$ : Speckle contrast on Delrin. $C_{\mathrm{d}}$ : Speckle contrast on scattering suspension.

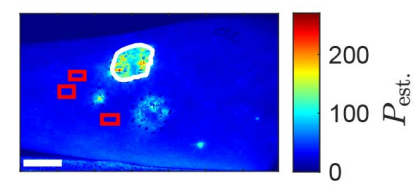

(a)

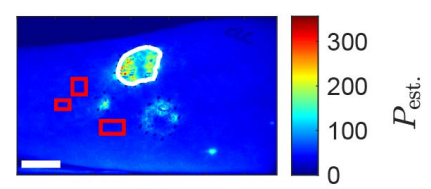

(b)

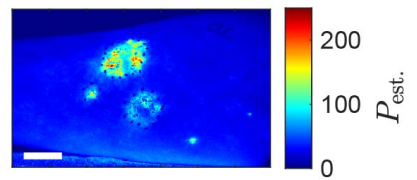

(c)

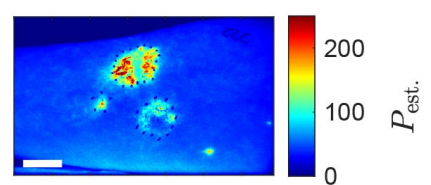

(d)

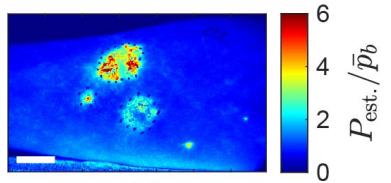

(e)

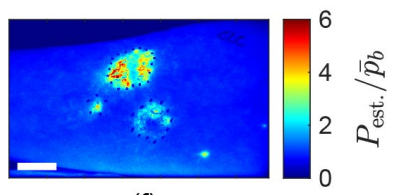

(f)

Fig. 5.14: Comparison of mounted (i.e. (a), (c) and (e)) and handheld (i.e. (b), (d) and (f)) measurements. $P_{\text {est. }}$ : estimated perfusion. Scale bars, $25 \mathrm{~mm}$. Red rectangles: manually selected regions for calculation of background perfusion $\left(\bar{p}_{b}\right)$. White polygons: manually selected lesion areas. Temporally averaged perfusion maps with (a-b) a localized color-map scaling and (c-d) the same color-map scaling. (e-f) Corresponding background corrected perfusion maps with the same color-map scaling. 


\section{Experiment index 7}

\begin{tabular}{ccccccc} 
& Time & $\%$ Aligned frames & $\overline{p_{b}}$ & $\bar{I}$ & $C_{\mathrm{s}}$ & $C_{\mathrm{d}}$ \\
\hline Mounted & $15: 39$ & 100 & 24 & 20.1 & 0.94 & 0.19 \\
\cline { 2 - 7 } Handheld & $15: 37$ & 100 & 34.4 & & &
\end{tabular}

Table 5.8: Detail information of the measurement pair. $\bar{p}_{b}$ : Average perfusion in the selected background areas. $\bar{I}$ : Mean intensity on Delrin out of 255. $C_{\mathrm{S}}$ : Speckle contrast on Delrin. $C_{\mathrm{d}}$ : Speckle contrast on scattering suspension.

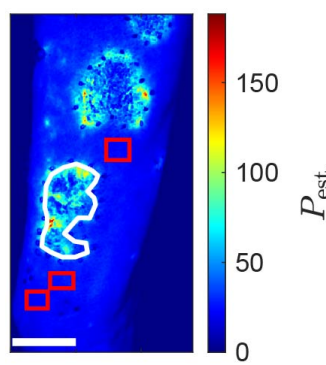

(a)

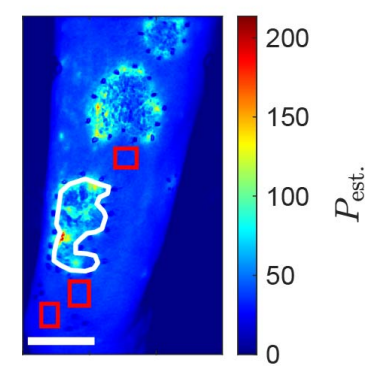

(b)

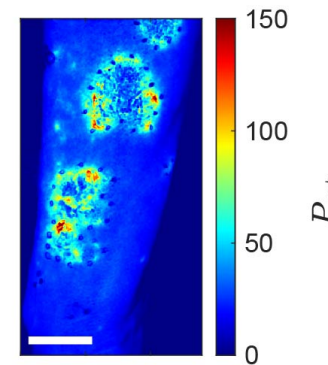

(c)

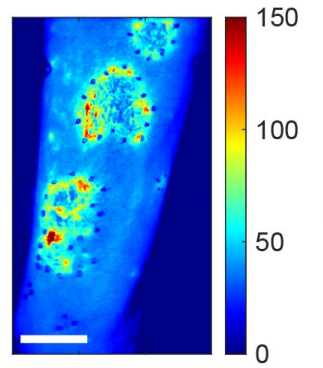

(d)

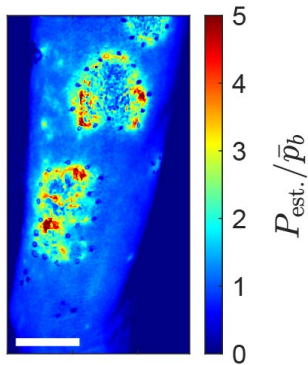

(e)

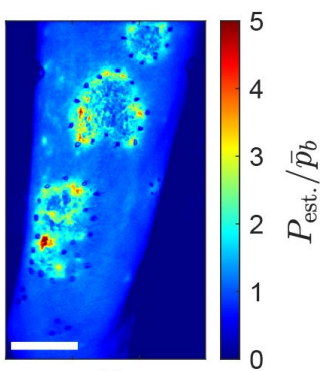

(f)

Fig. 5.15: Comparison of mounted (i.e. (a), (c) and (e)) and handheld (i.e. (b), (d) and (f)) measurements. $P_{\text {est. }}$ : estimated perfusion. Scale bars, $25 \mathrm{~mm}$. Red rectangles: manually selected regions for calculation of background perfusion $\left(\bar{p}_{b}\right)$. White polygons: manually selected lesion areas. Temporally averaged perfusion maps with (a-b) a localized color-map scaling and (c-d) the same color-map scaling. (e-f)

Corresponding background corrected perfusion maps with the same color-map scaling. 


\section{Experiment index 8}

\begin{tabular}{ccccccc} 
& Time & \% Aligned frames & $\overline{p_{b}}$ & $\bar{I}$ & $C_{\mathrm{s}}$ & $C_{\mathrm{d}}$ \\
\hline Mounted & $9: 26$ & 100 & 21 & 20.4 & 0.9 & 0.24 \\
\cline { 2 - 5 } Handheld & $9: 23$ & 100 & 27.2 & & &
\end{tabular}

Table 5.9: Detail information of the measurement pair. $\bar{p}_{b}$ : Average perfusion in the selected background areas. $\bar{I}$ : Mean intensity on Delrin out of 255 . $C_{\mathrm{s}}$ : Speckle contrast on Delrin. $C_{\mathrm{d}}$ : Speckle contrast on scattering suspension.

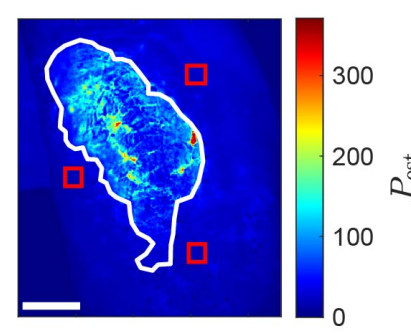

(a)

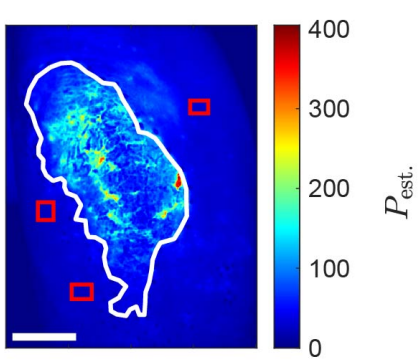

(b)

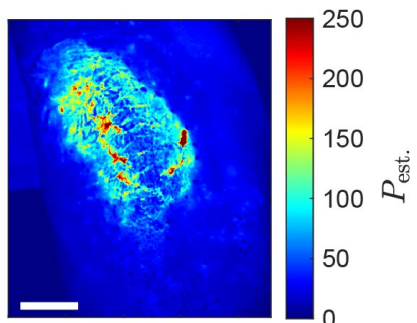

(c)

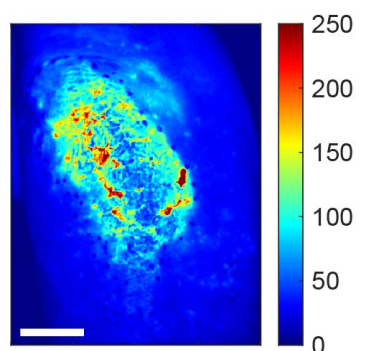

(d)

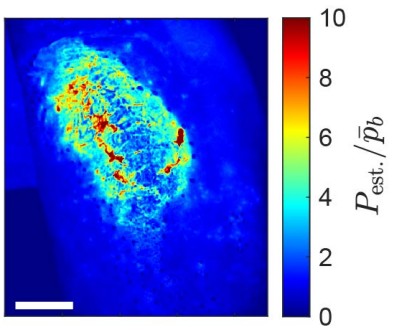

(e)

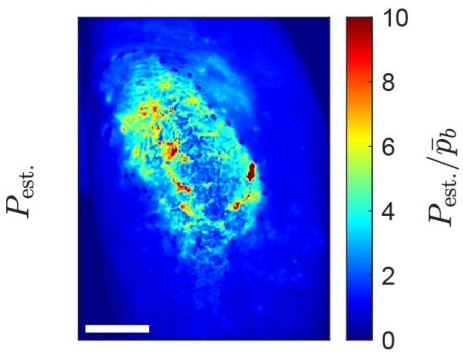

(f)

Fig. 5.16: Comparison of mounted (i.e. (a), (c) and (e)) and handheld (i.e. (b), (d) and (f)) measurements. $P_{\text {est. }}$ : estimated perfusion. Scale bars, $25 \mathrm{~mm}$. Red rectangles: manually selected regions for calculation of background perfusion $\left(\bar{p}_{b}\right)$. White polygons: manually selected lesion areas. Temporally averaged perfusion maps with (a-b) a localized color-map scaling and (c-d) the same color-map scaling. (e-f) Corresponding background corrected perfusion maps with the same color-map scaling. 


\section{Experiment index 9}

\begin{tabular}{ccccccc} 
& Time & $\%$ Aligned frames & $\overline{p_{b}}$ & $\bar{I}$ & $C_{\mathrm{s}}$ & $C_{\mathrm{d}}$ \\
\hline Mounted & $13: 16$ & 100 & 37.4 & 18.1 & 0.66 & 0.24
\end{tabular}

Table 5.10: Detail information of the measurement pair. $\bar{p}_{b}$ : Average perfusion in the selected background areas. $\bar{I}$ : Mean intensity on Delrin out of $255 . C_{\mathrm{S}}$ : Speckle contrast on Delrin. $C_{\mathrm{d}}$ : Speckle contrast on scattering suspension.

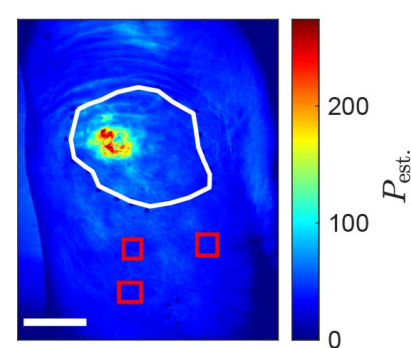

(a)

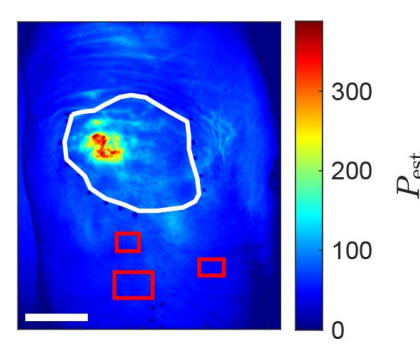

(b)

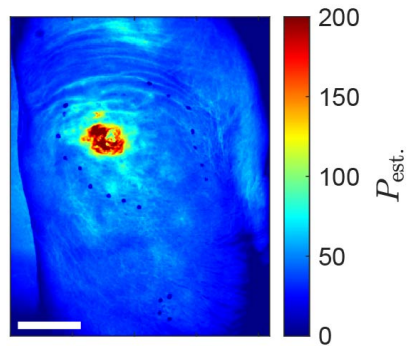

(c)

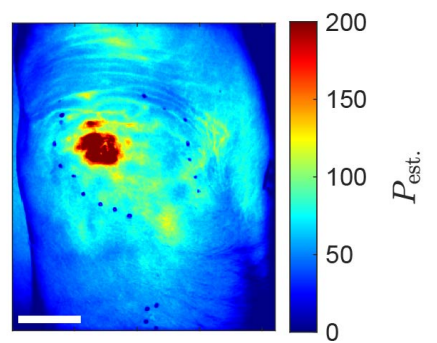

(d)

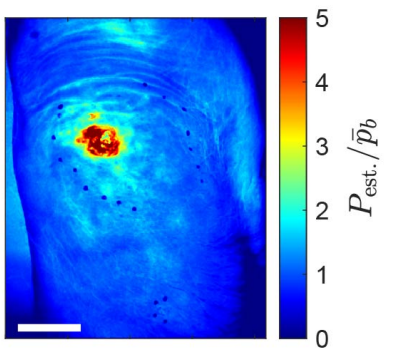

(e)

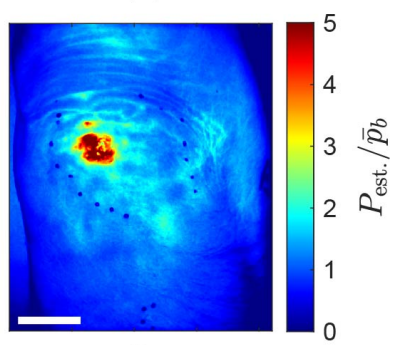

(f)

Fig. 5.17: Comparison of mounted (i.e. (a), (c) and (e)) and handheld (i.e. (b), (d) and (f)) measurements. $P_{\text {est. }}$ : estimated perfusion. Scale bars, $25 \mathrm{~mm}$. Red rectangles: manually selected regions for calculation of background perfusion $\left(\bar{p}_{b}\right)$. White polygons: manually selected lesion areas. Temporally averaged perfusion maps with (a-b) a localized color-map scaling and (c-d) the same color-map scaling. (e-f)

Corresponding background corrected perfusion maps with the same color-map scaling. 


\section{Experiment index 10}

\begin{tabular}{ccccccc} 
& Time & \% Aligned frames & $\overline{p_{b}}$ & $\bar{I}$ & $C_{\mathrm{s}}$ & $C_{\mathrm{d}}$ \\
\hline Mounted & $13: 25$ & 100 & 33 & & & \\
\cline { 2 - 6 } Handheld & $13: 23$ & 53 & 53.5 & & 0.64 & 0.2
\end{tabular}

Table 5.11: Detail information of the measurement pair. $\bar{p}_{b}$ : Average perfusion in the selected background areas. $\bar{I}$ : Mean intensity on Delrin out of $255 . C_{\mathrm{S}}$ : Speckle contrast on Delrin. $C_{\mathrm{d}}$ : Speckle contrast on scattering suspension.

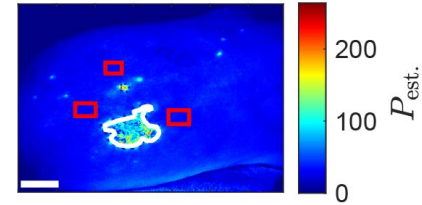

(a)

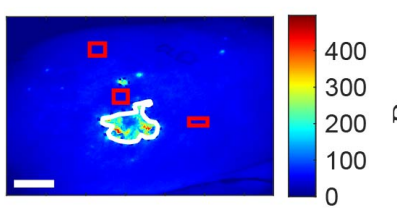

(b)

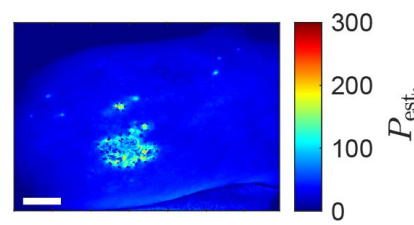

(c)

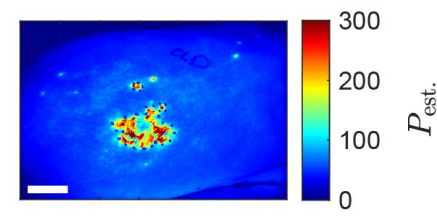

(d)

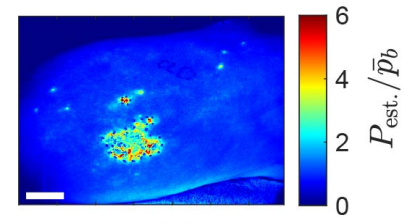

(e)

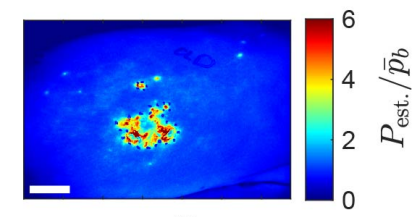

(f)

Fig. 5.18: Comparison of mounted (i.e. (a), (c) and (e)) and handheld (i.e. (b), (d) and (f)) measurements. $P_{\text {est. }}$ : estimated perfusion. Scale bars, $25 \mathrm{~mm}$. Red rectangles: manually selected regions for calculation of background perfusion $\left(\bar{p}_{b}\right)$. White polygons: manually selected lesion areas. Temporally averaged perfusion maps with (a-b) a localized color-map scaling and (c-d) the same color-map scaling. (e-f) Corresponding background corrected perfusion maps with the same color-map scaling. 


\section{Experiment index 11}

\begin{tabular}{|c|c|c|c|c|c|c|}
\hline & Time & $\%$ Aligned frames & $\overline{p_{b}}$ & $\bar{I}$ & $C_{\mathrm{s}}$ & $C_{\mathrm{d}}$ \\
\hline Mounted & $14: 24$ & 100 & 25.5 & & 085 & 022 \\
\hline Handheld & $14: 22$ & 57 & 36.5 & & & \\
\hline
\end{tabular}

Table 5.12: Detail information of the measurement pair. $\bar{p}_{b}$ : Average perfusion in the selected background areas. $\bar{I}$ : Mean intensity on Delrin out of 255. $C_{\mathrm{S}}$ : Speckle contrast on Delrin. $C_{\mathrm{d}}$ : Speckle contrast on scattering suspension.

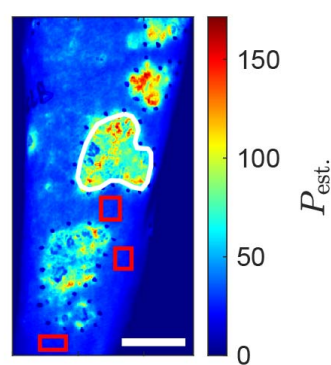

(a)

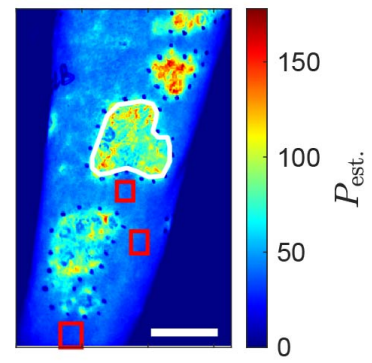

(b)

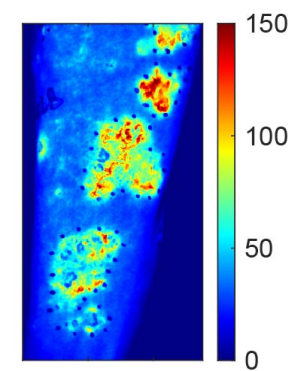

(c)

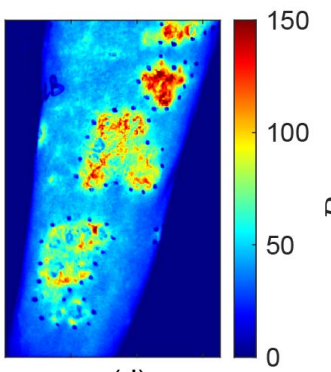

(d)

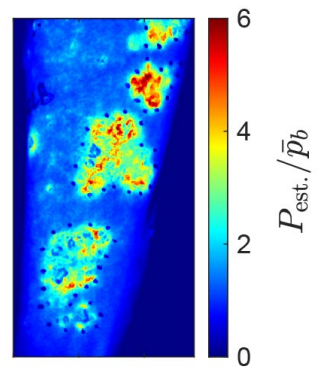

(e)

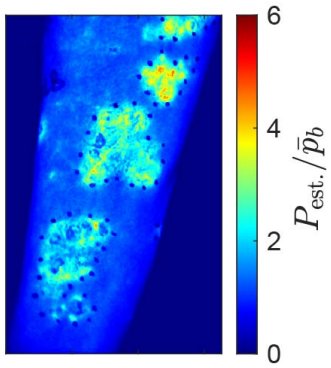

(f)

Fig. 5.19: Comparison of mounted (i.e. (a), (c) and (e)) and handheld (i.e. (b), (d) and (f)) measurements. $P_{\text {est. }}$ : estimated perfusion. Scale bars, $25 \mathrm{~mm}$. Red rectangles: manually selected regions for calculation of background perfusion $\left(\bar{p}_{b}\right)$. White polygons: manually selected lesion areas. Temporally averaged perfusion maps with (a-b) a localized color-map scaling and (c-d) the same color-map scaling. (e-f) Corresponding background corrected perfusion maps with the same color-map scaling. 


\section{References}

[1] M. Draijer, E. Hondebrink, T. van Leeuwen, and W. Steenbergen, "Review of laser speckle contrast techniques for visualizing tissue perfusion", Lasers in Medical Science 24, 639-651 (2008).

[2] D. A. Boas and A. K. Dunn, "Laser speckle contrast imaging in biomedical optics", Journal of Biomedical Optics 15, 011109 (2010).

[3] W. Heeman, W. Steenbergen, G. M. van Dam, and E. C. Boerma, "Clinical applications of laser speckle contrast imaging: a review”, Journal of Biomedical Optics 24, 1 (2019).

[4] K. Lee, "Diffuse speckle contrast analysis (DSCA) for deep tissue blood flow monitoring", Advanced Biomedical Engineering 9, 21-30 (2020).

[5] A. G. M. Hendriks, W. Steenbergen, E. Hondebrink, J. C. G. van Hespen, P. C. M. van de Kerkhof, and M. M. B. Seyger, "Whole field laser doppler imaging of the microcirculation in psoriasis and clinically unaffected skin", Journal of Dermatological Treatment 25, 18-21 (2013).

[6] A. G. M. Hendriks, P. C. M. van de Kerkhof, C. S. de Jonge, M. Lucas, W. Steenbergen, and M. M. B. Seyger, "Clearing of psoriasis documented by laser doppler perfusion imaging contrasts remaining elevation of dermal expression levels of CD31", Skin Research and Technology 21, 340-345 (2014).

[7] A. G. Hendriks, W. Steenbergen, P. L. Zeeuwen, J. Schalkwijk, E. Hondebrink, M. A. Klitsie, R. Schreurs, P. C. van de Kerkhof, and M. M. Seyger, "Perfusion intensity correlates with expression levels of psoriasis-related genes and proteins", Skin Pharmacology and Physiology 28, 296-306 (2015).

[8] L. Cooper, R. K. Chan, P. K. Bohan, A. H. Carlsson, and T. Everett, "642 the use of laser speckle contrast imaging in assessing depth and progression of burn wounds", Journal of Burn Care \& Research 41, S166-S167 (2020).

[9] M. B. B. Khashru, "Laser speckle contrast imaging, a feasible device for monitoring postoperative microcirculation: A case report", Journal of Orthopaedics and Sports Medicine 02 (2020).

[10] D. Briers, D. D. Duncan, E. Hirst, S. J. Kirkpatrick, M. Larsson, W. Steenbergen, T. Stromberg, and O. B. Thompson, "Laser speckle contrast imaging: theoretical and practical limitations", Journal of Biomedical Optics 18, 066018 (2013).

[11] A. Fercher and J. D. Briers, "Flow visualization by means of single-exposure speckle photography", Optics communications 37, 326-330 (1981).

[12] J. Zötterman, R. Mirdell, S. Horsten, S. Farnebo, and E. Tesselaar, "Methodological concerns with laser speckle contrast imaging in clinical evaluation of microcirculation", PLOS ONE 12, e0174703 (2017). 
[13] G. Mahé, P. Rousseau, S. Durand, S. Bricq, G. Leftheriotis, and P. Abraham, "Laser speckle contrast imaging accurately measures blood flow over moving skin surfaces", Microvascular Research 81, 183-188 (2011).

[14] G. Mahe, P. Abraham, A. L. Faucheur, A. Bruneau, A. Humeau-Heurtier, and S. Durand, "Cutaneous microvascular functional assessment during exercise: a novel approach using laser speckle contrast imaging", Pflügers Archiv - European Journal of Physiology 465, 451-458 (2013).

[15] L. Omarjee, I. Signolet, A. Humeau-Heutier, L. Martin, D. Henrion, and P. Abraham, "Optimisation of movement detection and artifact removal during laser speckle contrast imaging”, Microvascular Research 97, 75-80 (2015).

[16] B. Lertsakdadet, B. Y. Yang, C. E. Dunn, A. Ponticorvo, C. Crouzet, N. Bernal, A. J. Durkin, and B. Choi, "Correcting for motion artifact in handheld laser speckle images", Journal of Biomedical Optics 23, 1 (2018).

[17] B. Lertsakdadet, C. Dunn, A. Bahani, C. Crouzet, and B. Choi, "Handheld motion stabilized laser speckle imaging”, Biomedical Optics Express 10, 5149 (2019).

[18] A. Chizari, T. Knop, B. Sirmacek, F. van der Heijden, and W. Steenbergen, "Exploration of movement artefacts in handheld laser speckle contrast perfusion imaging", Biomedical Optics Express 11, 2352 (2020).

[19] A. Chizari, T. Knop, W. Tsong, S. Schwieters, and W. Steenbergen, "Influence of wavefront types on movement artefacts in handheld laser speckle contrast perfusion imaging”, OSA Continuum 4, 1875-1888 (2021).

[20] R. Heidenreich, M. Röcken, and K. Ghoreschi, "Angiogenesis drives psoriasis pathogenesis”, International journal of experimental pathology 90, 232-248 (2009).

[21] O. Frank, M. Nestle, H. Daniel, M. Kaplan, and J. Barker, "Mechanisms of disease: Psoriasis", N Engl J Med 361, 496-509 (2009).

[22] A. Murray, A. Herrick, T. Moore, T. King, and C. Griffiths, "Dual wavelength (532 and $633 \mathrm{~nm}$ ) laser doppler imaging of plaque psoriasis”, British Journal of Dermatology 152, 1182-1186 (2005).

[23] S. J. Kirkpatrick, D. D. Duncan, and E. M. Wells-Gray, "Detrimental effects of specklepixel size matching in laser speckle contrast imaging”, Optics Letters 33, 2886 (2008).

[24] R. Kesavamoorthy, T. Sakuntala, and A. K. Arora, "In situ measurement of charge on polystyrene particles in colloidal suspension", Measurement Science and Technology 1, 440 (1990).

[25] Jincheng Pang, "Localized Active Contour", https://www.mathworks.com/ matlabcentral/fileexchange/44906-localized-active-contour (2014), online; accessed 19 April 2020. 
[26] C. Li, C.-Y. Kao, J. Gore, and Z. Ding, "Minimization of region-scalable fitting energy for image segmentation", IEEE Transactions on Image Processing 17, 1940-1949 (2008).

[27] S. Lankton and A. Tannenbaum, "Localizing region-based active contours", IEEE Transactions on Image Processing 17, 2029-2039 (2008).

[28] A. Yezzi, A. Tsai, and A. Willsky, "A statistical approach to snakes for bimodal and trimodal imagery", in Proceedings of the Seventh IEEE International Conference on Computer Vision (IEEE) (1999).

[29] G. Evangelidis, "Iat: A matlab toolbox for image alignment", https://sites.google. com/site/imagealignment/ (2013).

[30] G. D. Evangelidis and E. Z. Psarakis, "Parametric image alignment using enhanced correlation coefficient maximization", IEEE Transactions on Pattern Analysis and Machine Intelligence 30, 1858-1865 (2008).

[31] J. W. Goodman, Speckle phenomena in optics: theory and applications (Roberts and Company Publishers) (2007).

[32] Z. Fegyver, "Calculate standard deviation: case of sliding window", http://matlabtricks.com/post-20/ calculate-standard-deviation-case-of-sliding-window (2013).

[33] J. D. Briers and S. Webster, "Laser speckle contrast analysis (lasca): a nonscanning, full-field technique for monitoring capillary blood flow", Journal of biomedical optics 1, 174-180 (1996).

[34] A. K. Dunn, H. Bolay, M. A. Moskowitz, and D. A. Boas, "Dynamic imaging of cerebral blood flow using laser speckle", Journal of Cerebral Blood Flow \& Metabolism 21, 195-201 (2001).

[35] L. M. Richards, E. L. Towle, D. J. Fox, and A. K. Dunn, "Intraoperative laser speckle contrast imaging with retrospective motion correction for quantitative assessment of cerebral blood flow", Neurophotonics 1, 015006 (2014).

[36] P. Zakharov, A. Völker, M. Wyss, F. Haiss, N. Calcinaghi, C. Zunzunegui, A. Buck, F. Scheffold, and B. Weber, "Dynamic laser speckle imaging of cerebral blood flow", Optics Express 17, 13904 (2009).

[37] K. Khaksari and S. J. Kirkpatrick, "Laser speckle contrast imaging is sensitive to advective flux", Journal of Biomedical Optics 21, 076001 (2016).

[38] D. D. Duncan and S. J. Kirkpatrick, "Can laser speckle flowmetry be made a quantitative tool?", JOSA A 25, 2088-2094 (2008). 


\section{Assessment of flow within developing chicken vasculature and biofabricated vascularized tissues using multimodal imaging techniques $* \dagger$}

Fluid flow shear stresses are important regulators of vascular organization and as such are potential candidates to guide vascular organization in engineered tissues. To include controllable organized vascular networks within engineered tissues, it is necessary to understand the fluid flow dynamics within vasculature of varying complexity. However, reported investigations of vascular organization and their associated flow dynamics within multiscale complex vasculature over time are limited, due to limitations in the available physiological pre-clinical models, and the optical inaccessibility and aseptic nature of these models. To overcome these limitations, this chapter uses LSCI, side-stream dark field (SDF) microscopy and white light imaging to investigate the structural and blood flow information of developing vascular networks within an ex ovo chicken embryo chorioallantoic membrane (CAM) model. First,

${ }^{*}$ Padmanaban, P. $^{+}$, Chizari, A. ${ }^{+}$, Knop, T., Zhang, J., Trikalitis, V.D., Koopman, B., Steenbergen, W., and Rouwkema, J. Assessment of flow within developing chicken vasculature and biofabricated vascularized tissues using multimodal imaging techniques. Scientific Reports, 11, 18251 (2021). https://doi.org/10.1038/s41598-021-97008-w ${ }^{+}$These authors contributed equally.

${ }^{\dagger}$ The multimode imaging experiments and incubator modification for PDMS based ex-ovo culture in this chapter are part of the present thesis. The fluid dynamic simulations and fabrication of the engineered tissue perfusion chambers are due to P. Padmanaban. 
white-light imaging is used to map the complete vascular network. Second, the spatial and temporal fluctuations of blood flow in the corresponding vessels are non-invasively captured by LSCI. Third, the organization of capillaries, as well as fluid flow velocities, are determined based on SDF microscopy, which enables the visualization of individual erythrocytes flowing through capillary networks. Based on this information, the fluid flow shear stresses within individual vessels are estimated by computational fluid dynamics simulations in order to get an understanding of the flow associated mechanical signals within developing vasculature. In proof-of-principle experiments, we perform LSCI on biofabricated perfusable muscle tissue models and show that LSCI is compatible with bioengineered tissues and can help to better understand vascular organization and flow perfusion. The application of LSCI and SDF on perfusable tissues enables us to study the flow perfusion in a non-invasive fashion. Flow manipulation helps to tune the vascular organization with multiscale vasculature into specific organization and to design mechanically stable tissues.

\subsection{Introduction}

The inclusion of multiscale vascular networks portraying a correct hierarchical organization within engineered tissues is essential for the tissue viability and function. Vascular networks that include large vessels and small capillaries are essential for transporting oxygen and nutrients to allow for tissue survival. Large vessels are needed to bridge distances without large pressure drops, whereas small capillaries are needed to access all cells within the tissue. Even though the field of biofabrication has progressed fast over the past years, fabricating a large tissue construct with a resolution down to the smallest capillaries $(\approx 5 \mu \mathrm{m})$ remains challenging. Therefore, a stable hierarchically organized vascular network including all relevant size scales, will likely still rely on tissue remodeling. As fluid flow is one of the key regulators of vascular organization and remodeling [1,2], it is important to understand the blood flow within developing vascular networks of varying complexity.

For a long time, vascular network formation during embryogenesis has been considered to be genetically predetermined [3]. However, more recent studies showed that vascular cells can display plasticity with respect to local cues such as hemodynamics, especially blood flow shear stresses, meaning that the organization is influenced by the environment [4]. Hemodynamics-driven vascular organization is vividly observable in the chick embryo where the arteries and veins appear just a few hours after the onset of the heartbeat and subsequently blood perfusion. Perturbation of blood flow within the naturally formed vascular network of the chick embryo has been used to observe the effect of hemodynamic changes on vascular organization [4-6]. Additionally, in vitro studies have shown that mechanical signals such as fluid flows [7, 8] and wall shear stresses $[9,10]$ play an important role in regulating the different stages of vascular 
organization. However, even though the importance of fluid flow related mechanical signals in a wide range of in vitro cellular organizational phenomena has been shown, it has not been possible so far to completely elucidate the physiological significance of these mechanical signals, largely due to a lack of accessible angiogenesis models and integrated imaging platforms fit for long-term culture.

Due to this, accessible angiogenesis models have become a key focus in tissue engineering. Multiple in vivo models exist for studying the vascular responses to biomaterials and drug testing. Examples include zebra fish [11-13], mice, skin flap windows $[14,15]$ and snake embryos $[16,17]$. These models are generally complex and are associated with ethical concerns. Moreover, these models provide only a small area for imaging and often biomaterials/drugs are tested at random locations, lacking spatiotemporal control. Due to these limitations, the shell-less ex-ovo culture of chick embryo and its chorioallantoic membrane (CAM) has become a popular model for studying vascular network organization [18-21].

Optical methods used for imaging flow within vascular networks include optical coherence tomography (OCT) [22], photoacoustics [23], ultrasound [24], bioluminescence [25] etc. OCT and photoacoustics provide three-dimensional images, however they suffer from limited field-of-view (FOV) and need a complicated experimental setup. To overcome these challenges, this study adopts laser speckle contrast imaging (LSCI) [26-28] and side-stream dark field (SDF) microscopy [29] to probe the spatial and temporal profile of blood flow distribution and erythrocyte velocities within individual capillaries. LSCI is noninvasive, requires a rather simple experimental setup and provides a wide FOV typically in the range of several square centimeters; however, it is a two-dimensional imaging modality. SDF also represents a simple, portable experimental setup with high sensitivity that provides fine, well-defined video recordings of capillary structures. Moreover, this modality uses light-emitting diodes (LED) as a light source rather than lasers as used in LSCI. The downside of SDF is that the probe covered by a disposable cap should touch the sample surface during imaging, which can result in perturbations of the developing tissue and causes concerns regarding the aseptic nature of cell and tissue culture.

In this study, we prepared an artificial eggshell in which chick embryos were cultured from day 3 to day 10 of development. The complete vasculature was imaged using color imaging and LSCI. The former was used to quantify vessel properties such as diameter while the later was used to explore the blood flow level of the vasculature at different locations and times. Additionally, LSCI experiments were performed on biofabricated muscle tissues containing a perfusable channel as a proof-of-concept (POC) to show the application of LSCI in engineered tissues. An SDF probe was used to visualize capillary structures and erythrocyte velocity on several locations of the vasculature. Figure 6.1 gives an overview of the three imaging modalities. To get a more quantitative understanding of the LSCI data, flow-phantom experiments 


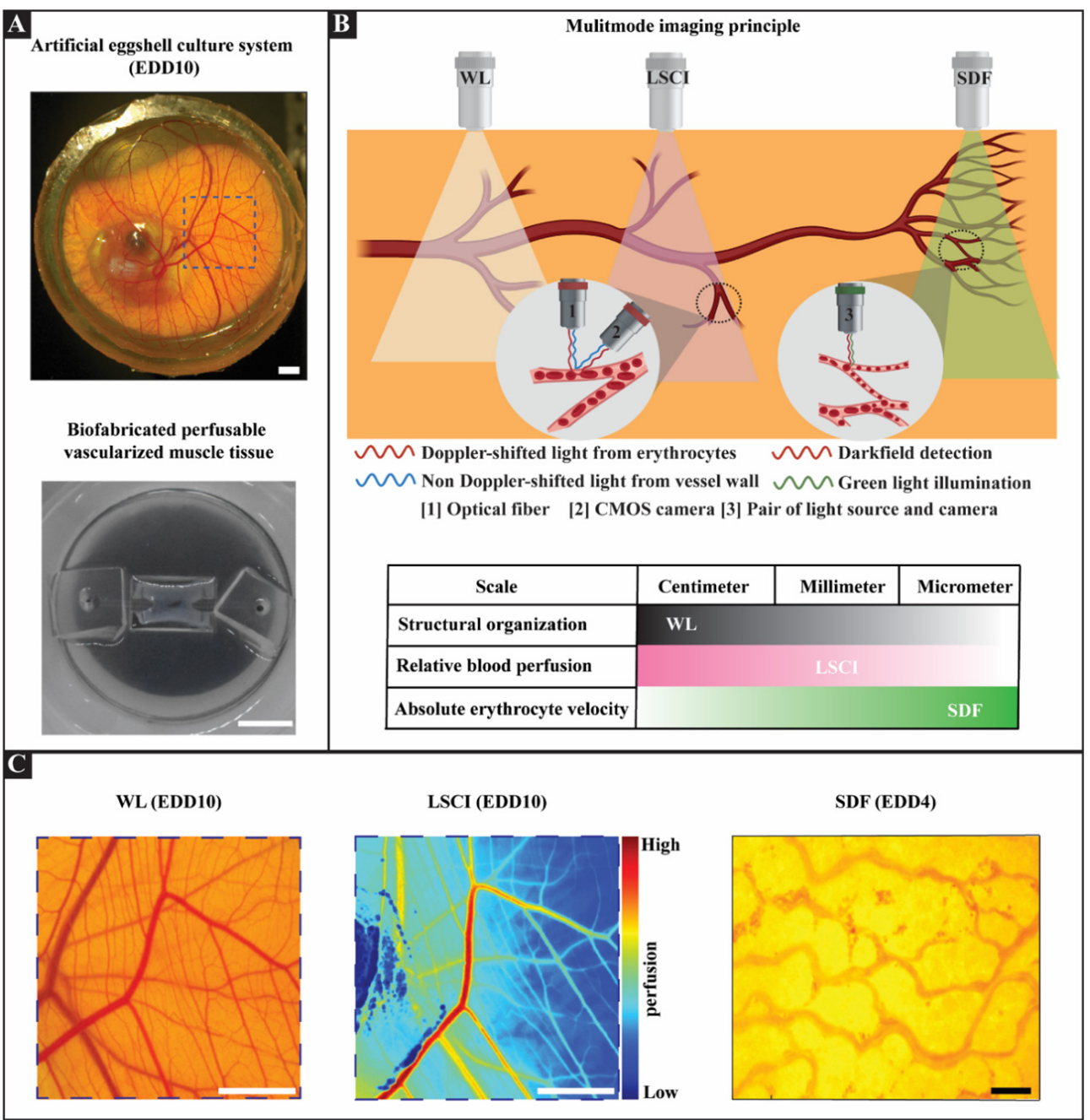

Fig. 6.1: Multimode imaging for probing vascular organization and associated flow dynamics. Panel A shows the artificial eggshell culture system with fully developed chick embryo of embryo development stage day 10 exhibiting multiscale vasculature of varying complexity, as well as a biofabricated vascularized tissue analogue. Both systems are compatible with WL, LSCI and SDF imaging methods and represent the proof-of-concept application towards engineering functional tissue constructs. Panel B shows the schematics explaining the principle of multiple imaging methods used in this study, with highlighted potential and limitations of the above-mentioned imaging methods. Panel $\mathbf{C}$ represents the examples of output results obtained from multiple imaging methods. Abbreviations: WL - white light (color) imaging; LSCI - laser speckle contrast imaging and SDF - side-stream dark field microscopy; EDD - embryo development day. White scale bars, $5 \mathrm{~mm}$. Black scale bar, $100 \mu \mathrm{m}$. The schematic figure in Panel B was created using Biorender.com. 
were carried out on microtubing of diameters ranging from $75-500 \mu \mathrm{m}$ through which a blood-mimicking scattering fluid (Intralipid with dye) was pumped. The microtubing diameters were comparable with diameters of the vasculatures imaged in in vivo experiments. The microtubing was mounted on both static scattering and static absorbing media to study their influence on the LSCI measurements. Additionally, computational fluid dynamics simulations (velocity-driven model) were performed to estimate shear stresses within the multiple vessel diameters.

\subsection{Methods}

\subsubsection{Fabrication of ex ovo culture system}

The culture system was made of polydimethylsiloxane, PDMS (Sylgard 184 silicone elastomer, Dow corning). The PDMS base was mixed at 10:1 (w/w) with the curing agent and poured into a laser cut circular shaped polymethyl methacrylate (PMMA) block. The mold was placed in a desiccator to remove the air bubbles, and it was cured overnight in a $65^{\circ} \mathrm{C}$ dry oven. Then, the cured PDMS and PMMA mold was carefully detached using a sharp razor blade.

\subsubsection{Culturing of chick embryos}

Fertilized chicken eggs (Dekalb white) were purchased from Het Anker bv (Ochten, The Netherlands) and stored at a temperature of $12^{\circ} \mathrm{C}$. $24 \mathrm{~h}$ prior to the egg transfer, the modified incubator was turned on and maintained at $38^{\circ} \mathrm{C}$ and $65 \%$ humidity throughout the entire culturing process (see Supplementary Fig. 6.8). For the first 3 days of incubation, the eggs were turned for 15 seconds every two hours to make sure that the embryo does not stick to the wall of the egg shell. After 3-day incubation period, the chicken embryo was transferred to the PDMS culture system in a sterile environment. As a first step, in order to avoid damage to the yolk and embryo vasculature, using sharp tweezers a hole of about $\approx 2 \mathrm{~mm}$ diameter was created on the eggshell and by inserting an $18 \mathrm{G}$ syringe microneedle $3 \mathrm{ml}$ of albumin was withdrawn using a plastic syringe. The removed albumin was carefully added to the culture system while limiting the introduction of air bubbles, after which the egg content was transferred.

\subsubsection{Color imaging and laser speckle contrast imaging of chick vascu- lature experiments}

For colour imaging, samples were illuminated using light emitting diodes (TYP b0913 Jansjö LED). A color camera (Basler acA1920-40uc USB3) was used for RGB imaging at full frame with the rate of $30 \mathrm{~Hz}$ and exposure time of $T=10 \mathrm{~ms}$. To 
realize an image resolution of up to $17.9 \mathrm{micron} /$ pixel a $25 \mathrm{~mm}$ camera objective (Pentax TV lens) was used. For a wider FOV and realizing image resolution of up to 55 micron/pixel, a $12 \mathrm{~mm}$ camera objective (FUJINON HF12XA-5M) was used. The maximum FOV for color imaging was $71.7 \times 73.1 \mathrm{~mm}^{2}$.

For LSCI, a continuous wave and coherent single longitudinal mode laser (CNI MSL-FN-671) with a wavelength of $\lambda=671 \mathrm{~nm}$ was used. The laser light was focused on a single mode optical fiber (Thorlabs P1-630A-FC-5) using an Olympus plan achromatic microscope objective (Thorlabs, RMS10X). A 20 degrees top hat square engineered diffuser (Thorlabs ED1-S20-MD) was located at the distal end of the optical fibre. The laser power illuminated from the optical fibre to the engineered diffuse was around $20 \mathrm{~mW}$. A monochrome camera (Basler acA2040 55um) recorded raw speckle frames with an exposure time of $T=10 \mathrm{~ms}$, frames rate of $50 \mathrm{~Hz}$, gain of $0 \mathrm{~dB}$ and acquisition time of 10 seconds. The sample at development day of 10 was imaged with both $T=5 \mathrm{~ms}$ and $T=10 \mathrm{~ms}$. Using a $25 \mathrm{~mm}$ camera objective (Pentax TV lens) with an effective numerical aperture of $F 8$, a spatial resolution of up to $15.4 \mathrm{micron} /$ pixel and a maximum FOV of $37 \times 38.4 \mathrm{~mm}^{2}$ was achieved. To suppress the background light, a hard coated bandpass interference filter of wavelength $675 \pm 12.5 \mathrm{~nm}$ (Edmund Optics) was placed in front of the monochrome camera objective. To suppress the effect of specular reflection from the laser light, a linear polarizer of wavelengths $600-1100 \mathrm{~nm}$ (Thorlabs LPNIRE100-B) with a direction perpendicular to the laser light polarization was mounted in front of the camera objective.

Each speckle frame was converted to a contrast map by applying a sliding window of size $7 \times 7$ pixels using sliding convolution technique. The equation for speckle contrast is [30]

$$
C=\frac{\sigma_{I}}{\bar{I}}
$$

where $\bar{I}$ and $\sigma_{I}$ are mean image value and image value fluctuations about the mean image value in the region of interest (ROI), respectively. With the assumption of a negative exponential form for the field autocorrelation function, the speckle contrast can be written as $[31,32]$

$$
C=\sqrt{\frac{\tau_{c}}{T}+\frac{\tau_{c}^{2}}{2 T^{2}}\left[\exp \left(-\frac{2 T}{\tau_{c}}\right)-1\right]}
$$

where $\tau_{c}$ and $T$ are correlation time and exposure time, respectively. The correlation time is assumed to be [33]

$$
\tau_{c}=\frac{\alpha}{v_{c}}=\frac{1}{P}
$$


where $v_{c}$ is the effective velocity and is called perfusion in arbitrary units. $\alpha$ is a constant and is set 1 in this study; thus, $v_{c}=P$. Based on the set exposure time $(T)$, a lookup table is made to assign a perfusion value $\left(P_{\text {est. }}\right)$ to each measured speckle contrast $\left(C_{\text {meas. }}\right)$ using linear interpolation. For the perfusion maps of Fig. 6.4(A) at each measurement, all frames were averaged to make a representative graph. For the perfusion maps of Fig. 6.5 at each measurement, $10 \%$ of the captured frames were selected for the averaging based on the maximum or minimum perfusion values at the ROI. The set colour bar scaling for each experiment shown in Fig. 6.4(A) and Fig. 6.5 corresponds to mean +7 times the standard deviation of the entire temporally averaged perfusion map. The data analysis for perfusion imaging is done in MATLAB R2019b.

\subsubsection{Side-stream dark field microscopy}

SDF uses LEDs (with a center wavelength of $530 \mathrm{~nm}$ ) as light source, present at the probe tip that directly penetrate deep into the specimen [29]. This wavelength corresponds to an isosbestic point in the absorption spectra of both deoxy- and oxyhemoglobin. The high absorption at this wavelength causes moving RBCs to appear black against a gray background in the SDF raw data. In order to give them a natural look we used a false color map ranging from red (associated with RBCs) to yellow (associated with the yolk). In SDF, the imaging system is surrounded by the light sources in a coaxial geometry. The dark field imaging arrangement avoids direct surface reflections. This way, clear images of the capillaries were captured without blurring. Due to low battery power consumption and portability, this technique can be used in different experimental scenarios.

A microscan video microscope system (Microscan Microvision Medical) was used for SDF microscopy with a spatial resolution of $1.4 \mathrm{micron} / \mathrm{pixel}$. The video microscope probe was mounted on a vertical motorized stage (Zaber X-LHM200AE03). The probe was gently moved toward the CAM vasculature until it touched the desired region. Then, the frames were acquired with a rate of $30 \mathrm{~Hz}$ for 12 seconds. The representative SDF image for each measurement was an average of 25 consecutive frames.

\subsubsection{Laser speckle contrast imaging of microtubing flow phantoms}

The laser illumination was as described in Subsection 6.2.3 with the difference that the diffuser was removed in order to provide higher intensity level. The CMOS camera type and its setting was as mentioned in Section 6.2.3. Here, instead of a camera objective, a $4-f$ imaging system of magnification $M \approx 1$ (image resolution of $3.3 \mathrm{micron} /$ pixel ) was built using two biconvex lenses (Thorlabs $f=75 \mathrm{~mm}$ ) and a diaphragm located at the focal point between the two lenses. The diaphragm was adjusted to make speckle size of approximately $3 \times 3$ pixels on the camera sensor. 
Table 6.1: Types of microtubing (MT) channels and the corresponding microsyringes (MS). ID: inner diameter; OD: outer diameter; vol.: volume; US: Upchurch Scientific; ANT: Autoclavable Nylon Tubing.

\begin{tabular}{c|c|c|c|c|c}
\multicolumn{1}{c}{ Channel No. } & MT type & MT ID $(\mu \mathrm{m})$ & MT OD $(\mu \mathrm{m})$ & MS type & MS vol. $(\mu \mathrm{l})$ \\
\hline \hline 1 & HPFA, US 1931 & 75 & 360 & ILS, 2100506 & 25 \\
2 & HPFA, US 1932 & 100 & 360 & ILS, 2100506 & 50 \\
3 & HPFA, US 1933 & 150 & 360 & ILS, 2100506 & 100 \\
4 & FEP, US 1688 & 200 & 800 & ILS, 2100506 & 100 \\
5 & PFA, US 1931 & 300 & 635 & ILS, 2100506 & 1000 \\
6 & ANT, US 1931 & 500 & 630 & ILS, 2100506 & 1000
\end{tabular}

In order to mimic the flow within the hollow structures of blood vessels, microtubing phantoms were used in the literature. This includes the use of dialyses tubes [34] along with the 3D printed tubes made up of soft materials [35]. In this study, six pairs of tubing channels were coupled to syringes (see Table 6.1 that also specifies the tube materials). The tubing channels were located on a static scattering object called Delrin and on a black tape (black anodized sheet). A wall was located between the Delrin plate and the black taping area to prevent light leakage (see Fig. 6.3(A)). To suppress the possible lens effect created by the wall curvature of tubing being imaged on the camera, microscope glass slides of $1 \mathrm{~mm}$ thickness (Thermo Scientific, Menzel-Glaser) were mounted on the tubing channels to form an enclosed space which was filled with ultrasound gel (Sonogel, Vertriebs GMBH). For creating a blood-resembling fluid in terms of optical properties at the wavelength of $\lambda=671$ nm, a stock solution of $830 \mu 1$ Intralipid 20\% (Fresenius Kabi Nederland BV) with reduced scattering coefficient of $26 \mathrm{~mm}^{-1}$ [36] and $43 \mu 1$ Ecoline 700 ink (Talens) with the measured absorption coefficient of $24.6 \mathrm{~mm}^{-1}$ and $10 \mathrm{ml}$ demineralized water was prepared. The volume fractions were chosen such that the prepared solution had a reduced scattering of $2 \mathrm{~mm}^{-1}$ and absorption coefficient of $0.1 \mathrm{~mm}^{-1}$ to mimic the scattering and absorption properties of blood at a wavelength of $\lambda=671 \mathrm{~nm}$ [37].

For each tube diameter, the coupled syringe was installed in a pump (Aladin-1000). The average flow speed (volumetric flux) through the tubing was calculated as

$$
V=\frac{F}{A}
$$

where $F$ and $A$ stand for flow rate and tubing area, respectively. Therefore, the dimension of $V$ is unit volume per unit time per unit area or simply unit distance per unit time. A fluid speed range of 0 to $7.5 \mathrm{~mm} / \mathrm{s}$ was applied for each experiment in 10 steps during which 250 raw speckle frames were recorded. Table 6.2 shows the set flow rates for each tube diameter.

To account for the effect of static scattering caused by any static scatterer con- 
Table 6.2: Flow rates for various tube diameters to obtain the same speed interval. $V_{\text {cal. }}$ : calculated volumteric flux; MT: microtubing; ID: inner diameter; $F_{\text {set }}$ : set flow rate.

\begin{tabular}{|c|c|c|c|c|c|c|c|c|c|c|c|}
\hline & & & & & & cal. (n & & & & & \\
\hline & & 0 & 0.5 & 1 & 2 & 3 & 4 & 5 & 6 & 7 & 7.5 \\
\hline & 75 & 0 & 0.1 & 0.3 & 0.5 & 0.8 & 1.1 & 1.3 & 1.6 & 1.9 & 2 \\
\hline & 100 & 0 & 0.2 & 0.5 & 0.9 & 1.4 & 1.9 & 2.4 & 2.8 & 3.3 & 3.5 \\
\hline & 150 & 0 & 0.5 & 1.1 & 2.1 & 3.2 & 4.2 & 5.3 & 6.4 & 7.4 & 8 \\
\hline $\mathrm{ID}(\mu \mathrm{m})$ & 200 & 0 & 0.9 & 1.9 & 3.8 & 5.7 & 7.5 & 9.4 & 11.3 & 13.2 & 14.1 \\
\hline & 300 & 0 & 2.1 & 4.2 & 8.5 & 12.7 & 17 & 21.2 & 25.5 & 29.7 & 31 \\
\hline & 500 & 0 & 5.9 & 11.8 & 23.6 & 35.3 & 47.1 & 58.9 & 70.7 & 82.5 & 88.4 \\
\hline & & & & & & $\operatorname{set}(\mu$ & & & & & \\
\hline
\end{tabular}

Table 6.3: Scattering ratio parameters set for various microtubing diameters in both scattering and absorbing background types. MT: microtubing; ID: inner diameter.

\begin{tabular}{|c|c|c|c|c|c|c|}
\hline & \multicolumn{6}{|c|}{ MT ID $(\mu \mathrm{m})$} \\
\hline & 75 & 100 & 150 & 200 & 300 & 500 \\
\hline Tube & 0.31 & 0.31 & 0.32 & 0.42 & 0.7 & 0.87 \\
\hline Tube on black & 0.47 & 0.47 & 0.63 & 0.71 & 0.84 & 0.9 \\
\hline
\end{tabular}

tributing to the observed dynamic speckle, the speckle contrast has the form [26]

$$
C=\sqrt{\beta\left[\rho^{2} \frac{\exp (-2 x)-1+2 x}{2 x^{2}}+4 \rho(1-\rho) \frac{\exp (-x)-1+x}{x^{2}}+(1-\rho)^{2}\right]}+C_{\text {noise }}
$$

where $x=T / \tau_{c}$ and $\beta$ is a constant that may be used for calibration. The scattering ratio parameter $\rho=I_{f} /\left(I_{f}+I_{s}\right)$ where $I_{f}$ is the time-averaged intensity of fluctuating dynamic scattered light and $I_{s}$ the intensity of the statically scattered light. For $\rho=1$ (i.e. fully dynamic scattering), Eq. (6.5) will be equal to Eq. (6.2) provided that $\beta=1$ and $C_{\text {noise }}=0$. Thus, for the sake of consistency, we set $\beta=1$ and $C_{\text {noise }}=0$. For each tube diameter a scattering ratio parameter is chosen such that a linear response of the perfusion values to the applied flow rates is achieved (see Table 6.3).

To obtain a fit for the measured speckle contrast versus perfusion with the Eqs. (6.3) and (6.5) for each tube diameter, speckle contrast from Eq. (6.5) was plotted versus a shifted and scaled version of the effective velocity of Eq. (6.3) as

$$
v_{c}^{\prime}=\alpha v_{c}+b
$$

The shifting and scaling parameters where chosen based on a visual fit with the measured data and the goodness of this fitting was examined by the $R^{2}$ value of a 
Table 6.4: List of fitting factors for theoretical fitting of the flow phantom experiments. MT: microtubing; ID: inner diameter.

\begin{tabular}{cccccccc} 
& & \multicolumn{7}{c}{$\operatorname{MT~ID~}(\mu \mathrm{m})$} \\
& & 75 & 100 & 150 & 200 & 300 & 500 \\
\cline { 2 - 8 } Tube on Delrin & $a$ & 70 & 120 & 340 & 550 & 990 & 2500 \\
& $b$ & 220 & 220 & -100 & -300 & -750 & -2200 \\
& $R^{2}$ & 0.89 & 0.86 & 0.67 & 0.88 & 0.99 & 0.99 \\
& & & & & & & \\
Tube on black & $a$ & 80 & 170 & 320 & 650 & 850 & 2000 \\
& $b$ & -80 & -100 & -300 & -450 & -450 & -1000 \\
& $R^{2}$ & 0.95 & 0.9 & 0.96 & 0.91 & 0.96 & 0.97
\end{tabular}

linear fitting of the estimated perfusion versus the actual fluid velocity (volumetric flux). See Table 6.4 for an overview of the fitting factors for the entire flow phantom experiments.

\subsubsection{Measurement of vascular network properties}

The color images of the chick vascular networks were acquired at the indicated development days as explained in Section 6.2.3, and the vascular network information such as vessel area, average vessel diameter, average vessel length, number of branching points or junctions and mean lacunarity were measured using ImageJ (Fiji) software and Angiotool plugin [38]. The image analysis pipeline is described in Supplementary Fig. 6.9. Graphs were plotted using OriginPro $2019 \mathrm{~b}$.

\subsubsection{Computational modeling and fluid dynamics simulations}

Computational models were used to calculate the fluid flow shear stresses of the developing vascular networks (for detailed information, see Supplementary Figure 6.10). Structural information such as microcapillary diameter calculated from SDF microscopy served as the geometrical input of the simulation model. Straight tubes that mimic blood vessels of varying diameter of 7-31 $\mu \mathrm{m}$ (see Figure 6.6(A) for the selected microcapillaries, highlighted with blue, green and red arrows) were designed and flow studies were performed using COMSOL Multiphysics software version 5.5. Boundary conditions include input flow velocities obtained from the SDF microscopy videos (for erythrocyte tracking, see Visualization 6.11 and Figure 6.6(B) for erythrocyte velocities) and zero pressure conditions at both the input and output 
were used. For this study, blood was assumed as a Newtonian fluid with the density $1060 \mathrm{~kg} / \mathrm{m}^{3}$ and dynamic viscosity 10-4 Pa.s. Even though blood is in essence a non-Newtonian liquid, the non-Newtonian properties of chicken blood were shown to be limited compared to human blood [39]. Flow type was considered as laminar and a vessel length of $1 \mathrm{~mm}$ was used.

\subsubsection{Biofabricated perfusable muscle tissue constructs}

Muscle tissue perfusion chambers (see Figure 6.7) were replica molded using PDMS, similar to section 6.2.1. The dimensions of the chambers were $8 \mathrm{~mm}$ in length, $5 \mathrm{~mm}$ in width and $2 \mathrm{~mm}$ in depth. Culture chambers were supplied with nylon wires $(0.5 \mathrm{~mm}$ diameter) before the chambers were filled with hydrogel. Mouse myogenic $\mathrm{C}_{2} \mathrm{C}_{12}$ cells were obtained from ATCC and cells were used up until passage number 21. Myoblasts were cultured in Dulbecco's modified Eagle's medium (DMEM) supplemented with $10 \%$ fetal bovine serum and $1 \%$ penicillin-streptomycin. Green fluorescent protein (GFP) expressing human umbilical vein endothelial cells (HUVECs) were obtained from Cellworks and cells were used up until passage number 5. GFP-HUVECs were cultured in EGM-2 medium (EGM-2 Basal medium + EGM-2 Supplements) with $1 \%(\mathrm{v} / \mathrm{v})$ Pen/Strep. All cells were maintained in a humidified incubator kept at $37^{\circ} \mathrm{C}$ and $5 \% \mathrm{CO}_{2}$ and were passaged upon reaching $80 \%$ confluency. A-cellular controls were prepared using gelatin. $5 \%$ gelatin hydrogel solution was prepared by mixing Type A 300 bloom porcine skin gelatin (G1890-500G, Sigma Aldrich) with demineralized water and stirred at $200 \mathrm{rpm}$ at $65^{\circ} \mathrm{C}$ for 30 minutes. Fibrin hydrogel was prepared by mixing $0.5 \mathrm{U} / \mathrm{ml}, 1 \mathrm{mg} / \mathrm{ml}$ and $6 \mathrm{mg} / \mathrm{ml}$ of thrombin, $\mathrm{CaCl}_{2}$ and fibrinogen respectively. $\mathrm{C}_{2} \mathrm{C}_{12}$ cells were resuspended in culture medium and were then mixed with fibrin hydrogel to reach a final cell concentration of $1.5 \times 10^{7}$ cells $/ \mathrm{ml}$ and a final fibrinogen concentration of $6 \mathrm{mg} / \mathrm{ml} .100 \mu \mathrm{l}$ of this mixture was then transferred to the perfusion chamber. After gelification, culture medium was added and the samples were incubated at $37^{\circ} \mathrm{C}$ and $5 \% \mathrm{CO}_{2}$. The nylon wires were removed after 7 days, resulting in a perfusable channel. The channels of select samples were seeded with $3.5 \times 10^{7}$ cells $/ \mathrm{ml}$ of GFP-HUVECs for 24 hours. LSCI was performed on day 8 .

\subsection{Results and discussion}

\subsubsection{Color imaging of chick vascular network organization}

Figure 6.2(A) illustrates color images of the entire vasculature during 5 development days, namely 3-6 and 10. Since the culture system allows for a wide view of the vasculature from the top side, multiple ROIs are selected in order to perform statistical analysis (See Figure 6.2(B)). The quantification metrics were performed as explained 
in Section 6.2.6 and Supplementary Figure 6.9. The results show an increasing trend in the vasculature area and number of branching points over time. The mean lacunarity (i.e. the void spaces in the image) were decreased over time, due to the increasing vascular sprouting and emergence of new vascular structures. An interesting trend was observed for the vessel diameter. Between EDD3 and EDD6, the average vessel diameter increased, which was accompanied by a wider spread in the vessel diameters. This points to a remodeling vascular network that becomes more multi-scale over time. On EDD10, the average vessel diameter was slightly decreased again, which may indicate a further stabilization of the formed vascular network. This is corroborated by the data on the average vessel length, which decreased between EDD5 and EDD10.

\subsubsection{Laser speckle contrast perfusion imaging}

\section{Microtubing flow phantoms}

The schematic diagram of the flow phantom study is depicted in Figure 6.3(A). For a comparison between the inner and outer diameters of the microtubings see Figure 6.3(B) that visualizes the cross sections. For the experiments on each tubing, three regions were selected namely, Delrin, tube on black and tube on Delrin. The mean intensity of these areas detected on the camera sensor is shown in Supplementary Figure 6.11(A-C), respectively.

The measured speckle contrast versus the set volumetric flux on these regions for the representative tube diameter of $150 \mu \mathrm{m}$ is depicted in Figure 6.3(C). It is observed that the measured speckle contrast on Delrin remains constant independent of the applied volumetric flux, which means that it acts as a baseline signal validating the stability of the light source during the measurement. For all volumetric fluxes, the drop in the measured speckle contrast for the case of tube on black was higher than the case of tube on Delrin. This observation is the case for all of the tube diameters used in this study (see Supplementary Figure 6.11(D-H)). For the case of tube on Delrin, the observed (backscattered) light includes contribution of light waves that (1) only propagate through the flow (experience Doppler shift); (2) only propagate through Delrin (static medium, no Doppler shift); (3) propagate through both the flow and Delrin. The cases (1) and (3) form dynamic speckle patterns of limited correlation time and the case (2) forms static speckle pattern of long correlation time (unity contrast). The speckle pattern is observed on the camera will be the result of superposition of both Doppler shifted and non-Doppler shifted light. However, for the case of tube on black, there is no contribution of light waves that propagate through a static background (Delrin) and in an ideal case (no static scattering material) fully dynamic speckle patterns are observed. Therefore, due to the presence of a partly static speckle pattern in the case of tube on Delrin, the observed speckle patterns will be of higher contrast (less blurred) than the case of tube on black. For a video of this 


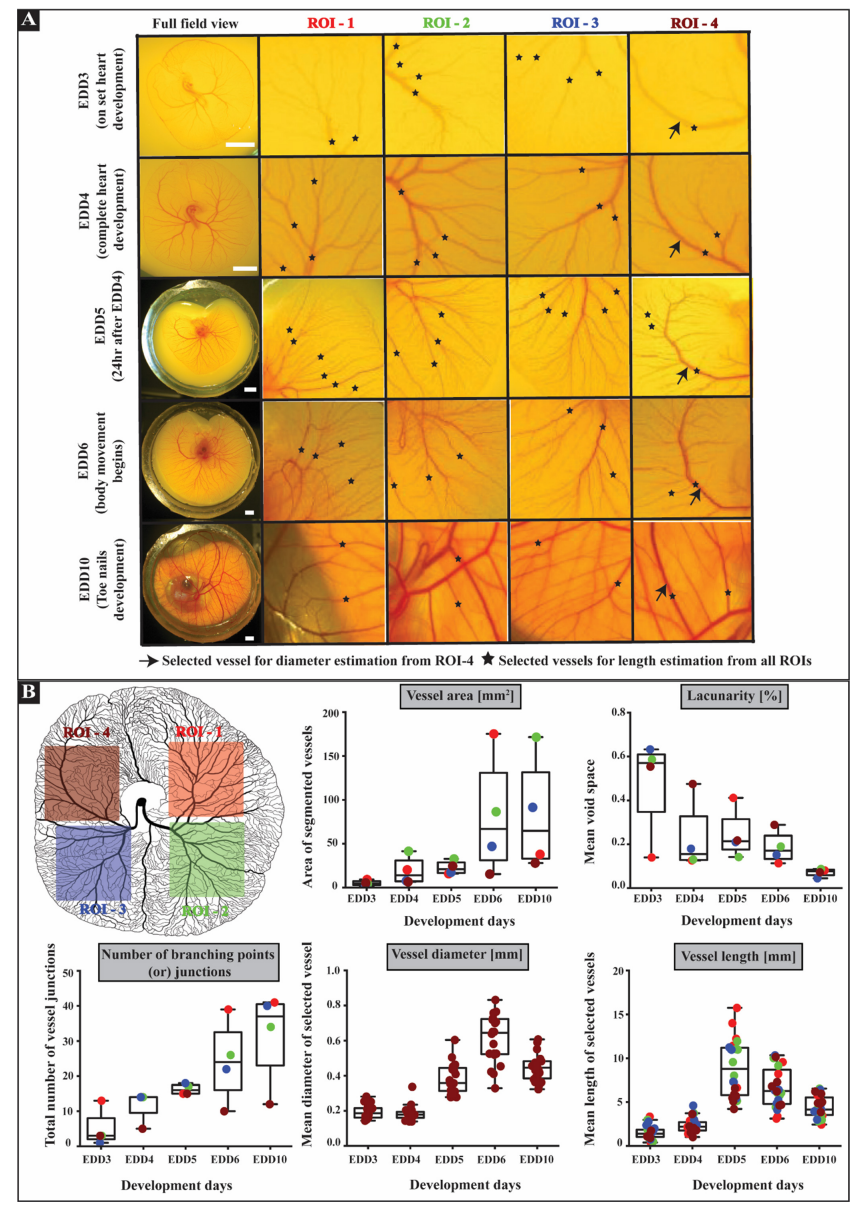

Fig. 6.2: Organization of developing vascular networks. Panel A shows the snapshots of entire vascular networks of developing chick embryo including the CAM cultured within artificial eggshell culture system. The figures represent the developmental stages of day 3-6 and day 10, displaying the evolution of vascular network organization in both space and time. For videos, see Visualization 6.11. Scale bars represent $5 \mathrm{~mm}$. Panel B represents the schematic figure of chick vascular network of the CAM development day 5 with highlighted ROIs, which are used for reference in Panel A and for the quantitative analysis. These multiple ROIs can be used for performing statistical analysis. Measured values are calculated from one sample $(n=1)$ and represented as mean \pm standard deviation along with individual data points. Measured values are compared within 4 different ROIs for different development days as indicated. Vessel diameter are calculated from single vessel of ROI-4 for different development days (highlighted with black arrows) and vessel length are calculated from multiple vessels from all ROIs (highlighted with black asterisks). For detailed explanation about quantitative analysis, see Section 6.2.6 and Supplementary Fig. 6.9. Schematic figure in Panel B was created using Adobe Illustrator 2020. 
situation see Visualization 6.7 in which black and white regions correspond to tube on black and tube on Delrin, respectively.

In Figure 6.3(E) the influence of tube diameter is probed for the case of tube on black. By increasing the volumetric flux, the measured speckle contrast decreases. For a certain volumetric flux, increasing tube diameter causes a higher drop in the speckle contrast. Similar behavior is observed for the case of tube on Delrin (see Supplementary Figure 6.11(I)). The underlying reason can be explained by considering intensity fluctuations at each camera pixel within the exposure time. The frequency of the observed intensity fluctuations obeys the optical Doppler effect [40]. The longer the tube diameter, the more the probability of multiple Doppler scattering. A higher Doppler shift causes more fluctuations within the exposure time and therefore more blurred speckle patterns of lower contrast.

The theoretical model introduced in Eqs. (6.3) and (6.5) estimates perfusion (effective velocity) as a function of exposure time and scattering ratio parameter $\rho$. Figure 6.3(D) shows the curves of speckle contrast versus perfusion for different values of scattering ratio parameter. For a certain perfusion value, a higher scattering ratio corresponds to a lower speckle contrast. The tube material, inner diameter, thickness and scattering background influence the scattering ratio parameter. To account for these effects and as explained in Section 6.2.5, the scattering ratio parameter for each tube diameter and scattering background was chosen such that the estimated perfusion responded linearly to the applied flow.

As a demonstration of the fitting of the measured data with the theory showed in Figure 6.3(D), the speckle contrast was plotted versus perfusion according to the scattering ratio parameter for each tube diameter showed in Table 6.3. Then, the measured speckle contrast data points were plotted versus a shifted and scaled version of the perfusion such that they matched with the theoretical curves. Figure 6.3(F) shows the result of the fitting for the case of tube on black. For the case of tube on Delrin see Supplementary Figure 6.11(J). The purpose of this fitting is to show that the measured data points match the behavior of the theoretical model, which is a way of validating the proper choice of the scattering ratio parameters. However, we acknowledge that the scaling and shifting parameters may not have a straightforward relation with the physical aspects such as speckle size and imaging system.

The estimated perfusion versus the applied volumetric flux for various tube diameters and the case of tube on black is shown in Figure 6.3(G). Although the estimated perfusion, to a good extent, is proportional to the applied volumetric flux, the slopes of the fitted lines depend on the tube diameter. Similar results are obtained for the case of tube on Delrin (see Supplementary Figure 6.11(K)). Notice that the estimated perfusion is a direct interpolation of the measured speckle contrast according to Eqs. (6.3) and (6.5); therefore, no scaling and shifting is applied. The estimated perfusion versus the applied flow rate for the case of tube on black is depicted in Figure 6.3(H). Now, 


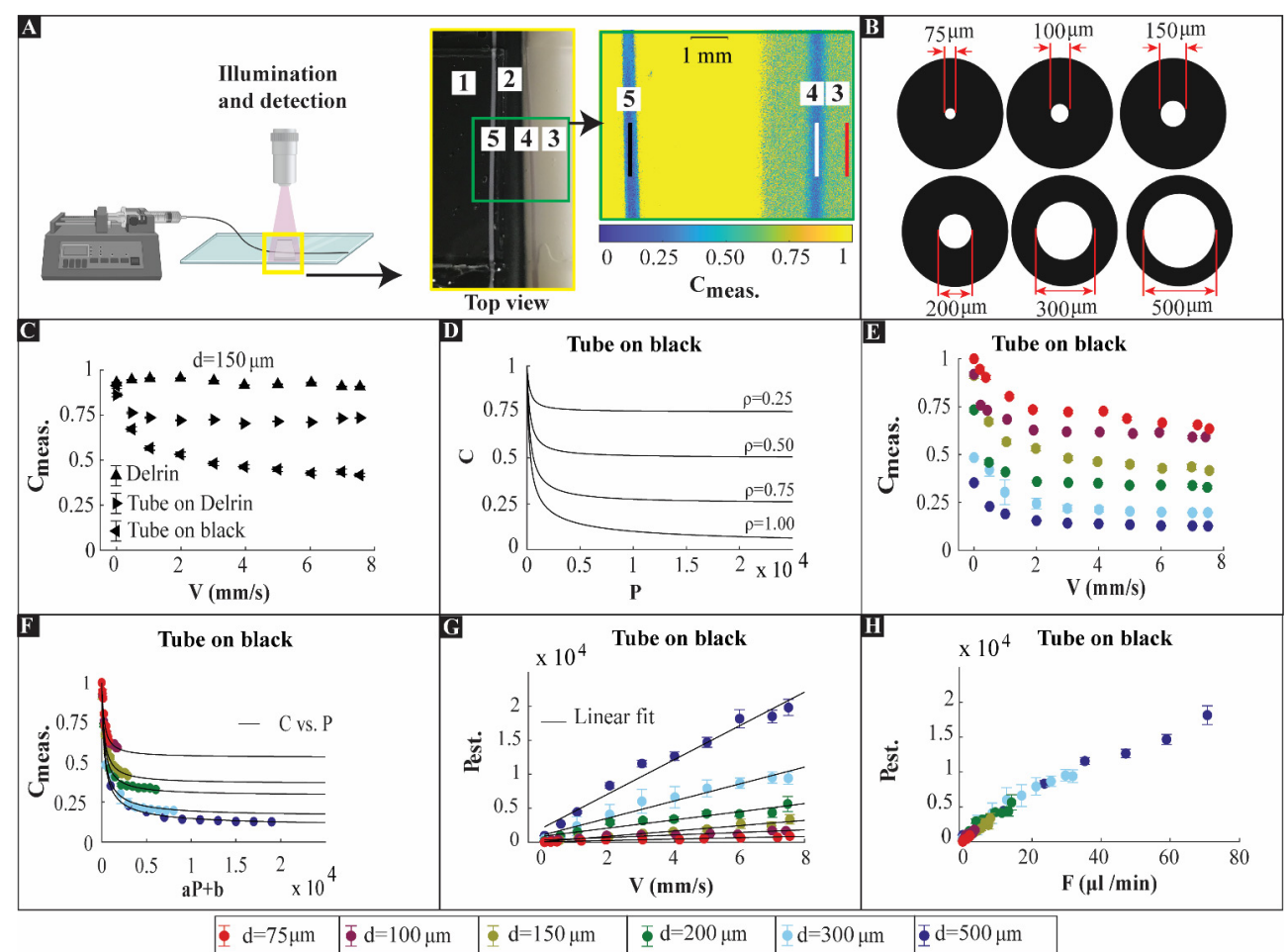

Fig. 6.3: LSCI on microtubing flow phantoms. Panel A, left, schematic of syringe pump connected to a microtubing for Intralipid-dye perfusion and laser speckle contrast measurement. Middle, top-view of the area for imaging. 1, glass plate mounted on microtubing and static media. 2, light isolating wall. 3, Delrin. 4, tube on Delrin. 5, tube on black. Right, representative speckle contrast map for the channel of $d=300 \mu \mathrm{m}$ at $F=17 \mu \mathrm{l} / \mathrm{min}$. The red, white and black ROIs indicate the regions in which $C_{\text {meas. }}$ are calculated, respectively. The schematic figure in Panel A is created using Biorender.com. Panel B shows the microtubings of different diameters, representing the blood vessel diameter variation of the CAM. Panel C shows the measured speckle contrast (calculated based on Eq. (6.1)) versus the volumetric flux (calculated based on Eq. (6.4)) for the representative tube diameter of $150 \mu \mathrm{m}$ as a comparison between the cases of Delrin, tube on Delrin and tube on black, respectively. Data points are mean \pm standard deviation. Panel D illustrates relation between the speckle contrast and the perfusion shown in Eqs. (6.3) and (6.5) for various scattering ratios. Panels E-H correspond to the case of tube on black and the colored data points represent the different vessel diameters as stated in Panel H. Panel E illustrates the measured speckle contrast versus the volumetric flux. Panel F illustrates the measured speckle contrast versus the shifted and scaled versions of the perfusion for each tube diameter overlapped with the theoretical curves of the speckle contrast versus perfusion. Panel G illustrates the estimated perfusion versus the volumetric flux with a linear fit for each tube diameter. Panel $\mathbf{H}$ is a plot of the estimated perfusion versus the flow rate. 
as well as the proportionality, the estimated perfusion shows independency to the tube diameter, when plotted versus the applied flow rate. Similar results are achieved for the case of tube on Delrin (see Supplementary Figure 6.11(L)). Our analysis suggests that knowledge about the tube diameter is crucial in making perfusion estimation that is proportional to the applied volumetric flux or flow rate. Moreover, vessel diameter is an important geometrical parameter that constantly changes over the development and varies within the chick vascular system.

\section{Blood flow distribution within vasculature of varying complexity}

The procedure of creating perfusion maps was described in Section 6.2.3. Temporally averaged perfusion maps of developing chicken vasculature on days 3-6 are shown in Figure 6.4(A). The spatiotemporal perfusion fluctuations within 10 seconds of recording on days 3-6 and 10 are visualized in Visualization 6.8. One vessel of this sample is chosen for probing the perfusion fluctuations over developing days of 3-6. At each day, average temporal fluctuations at three locations of the selected vessel are calculated. Figure 6.4(B) shows an overview of the average temporal fluctuations over days as well as representative temporal fluctuations for days 3 and 4 . Note that speckle contrast maps gathered on EDD10 with an exposure time of $10 \mathrm{~ms}$ were highly blurred due to the high blood flow within the samples combined with embryo movement artefacts. An alternative measurement with an exposure time of $5 \mathrm{~ms}$ was carried out which may preclude a comparison with EDD3-6. Therefore, this data is not included in the analysis of this Subsection. More recently, it was shown that movement artefacts caused by a moving embryo can be corrected using an optical flow algorithm but that requires simultaneous RGB imaging using a color camera [41].

For each EDD, the average estimated perfusion of the vessel decreases as the distance from the heart increases. This is due to the supply of blood to smaller vessels and capillaries on its way. An increase in the average estimated perfusion of all three regions from days 3 till 5 is also observed. The ratio of the average estimated perfusion on the region closest to the heart (regions $\gamma$ in Figure 6.4(A)) on day 5 compared to day 3 is calculated as 7 . The underlying reason is the growth of the sample in terms of heart capacity, blood vessel numbers and generation of more blood. The heart shows the highest estimated perfusion level within the temporally averaged perfusion maps. In Figure 6.4(C) the heart areas within days 3-5 are illustrated. The heart rate over developing days is calculated by counting the number of peaks in the temporal fluctuations of the estimated perfusion. For this sample, it increases from 90 beats per minute (bpm) on day 3 to $228 \mathrm{bpm}$ on day 10 . 


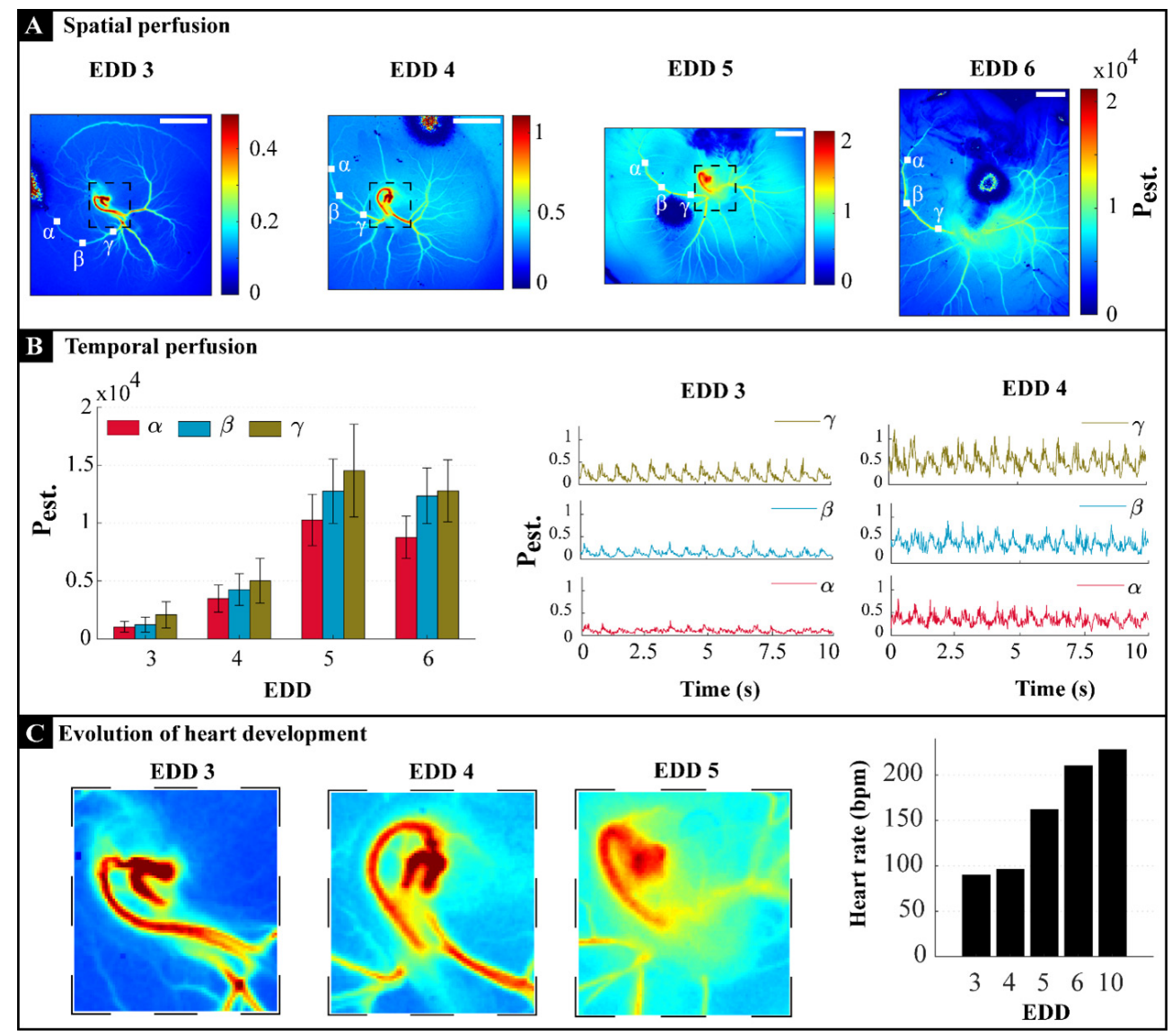

Fig. 6.4: Blood flow distribution of developing vascular networks. Panel A shows the average spatial perfusion maps of complete vascular network of chick embryo belonging to developmental day 3 till 6 . Scale bars represent $5 \mathrm{~mm}$. EDD, embryo development day. $P_{\text {est. }}$, estimated perfusion. Panel B shows the temporal perfusion profile from the regions in Panel A (highlighted using Greek symbols $\alpha$ :alpha, $\beta$ :beta and $\gamma$ :gamma). Measured perfusion values are obtained from single independent biological sample $(n=1)$ over a time frame of 10 seconds. Data of bar graph are mean \pm standard deviation. Panel $\mathrm{C}$ represents zoomed snapshot of heart development from day 3 till 5 (highlighted regions in panel A using dashed boxes) and the heart rate of the sample. bpm, beats per minute. 


\section{Arterial-venous flow of developing vascular networks}

As described in Section 6.2.5 temporally averaged perfusion maps are created by the selection of perfusion maps at certain time points in order to visualize arterial and venous flow maps. For EDD3, a region $x$ on a vessel close to heart (see Figure 6.5(A)) is chosen to extract the temporal fluctuations of the estimated perfusion. Then, by choosing the frames correspond to maximum (systole) and minimum (diastole) values of region $x$, arterial and venous flow maps are formed. See Visualization 6.9 that demonstrates the two flow maps in an overlapping manner. The arterial flow map shows the spread of the vessels supplied by the main arteries throughout the vascular network while the venous flow map shows high perfusion on the heart, a large vessel below it and the upper boundary of the vascular network. In Figure 6.5(B), the same analysis is performed for EDD4. The mean estimated perfusion on the regions $x$ and $y$ for the development days 3 and 4 are compared in Figure 6.5(C) for both the systole and diastole cycles. For the systole cycle on region $x$, the mean estimated perfusion ratio of day 4 to 3 is 2.3 , while this ratio is 1.2 on region $y$.

On EDD10, a fluctuation corresponding to the heartbeat pattern of the embryo is observed on the main vein labeled as region $y$ (see Figure 6.5(D)). The temporal fluctuations of the estimated perfusion on region $x$ that corresponds to an arterial vessel is more stabilized than the region $y$. Here, the arterial and venous flow maps are formed by temporal averaging of the perfusion maps that belong to the minimum (diastole) and maximum (systole) points of the region $y$. The main vein labeled with the white arrows in Figure 6.5(D) generates a higher signal during the venous flow. Based on white light imaging, this vessel has a darker red color compared to the arterial vessels which can be seen in Figure 6.1(C, left), indicating that the blood flowing through the vessel is less oxygenated and therefore venous. This observation can be explained as follows. The vessel labeled with $y$ collects all the deoxygenated blood from the vasculature and directly reaches the heart, such that it moves slightly with each pulsatility of the heart (See Visualization 6.6 EDD10). However, the vessel indicated with $x$ is one of the many arterial branches that carry oxygenated blood. As a result, $x$ consists of less blood flow and rhythmic pulsatility pattern compared with $y$.

\section{Erythrocytes visualization within individual blood vessels using SDF microscopy}

SDF microscopy as explained in Section 6.2.4 was performed on multiple locations of the vascular network on EDD4. Figure 6.6(A) shows the temporally averaged images of different locations namely, vascular crowded region, close to heart, away from the heart and at the boundaries of the vascularized membrane. For videos of moving erythrocytes within multiple locations and their associated measurements see Visualization 6.10 and Visualization 6.11, respectively. The SDF images were used to elucidate the capillary structure as well as the movement of individual erythrocytes. 


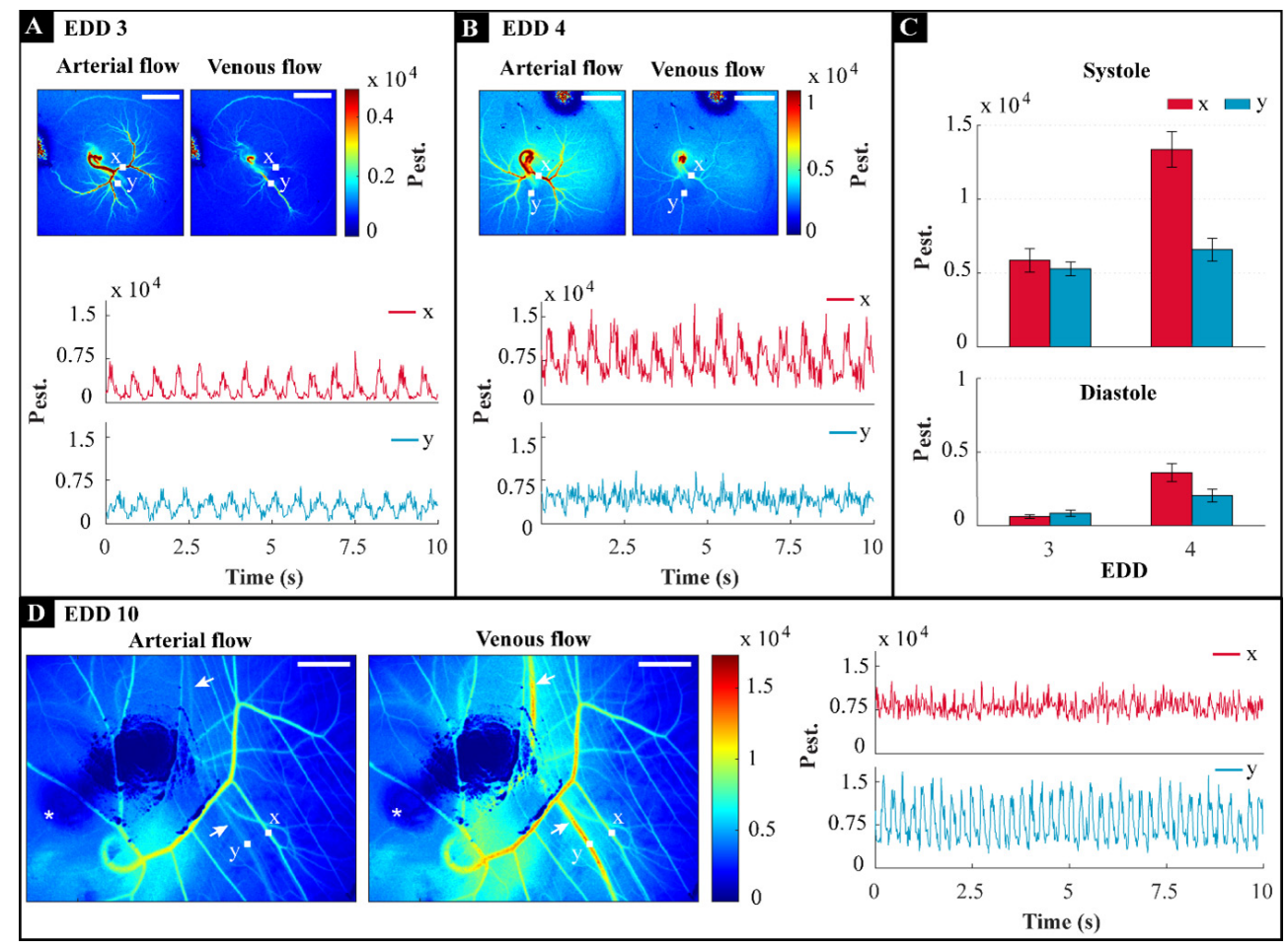

Fig. 6.5: Blood flow distribution within arteries and veins. Panel A, perfusion maps based on averaging of a number of frames chosen during systole (maximum perfusion) and diastole (minimum perfusion) from the region $x$ that represent arterial and venous flow, respectively, for the development day 3. EDD, embryo development day. $P_{\text {est. }}$, estimated perfusion. Scale bars, $5 \mathrm{~mm}$. Panel B represents the same analysis as Panel A but for the development day 4. Panel C, a comparison between the averaged perfusion maps (mean \pm standard deviation) for both systole and diastole during days 3 and 4 of the development. $x$ and $y$ correspond to the regions shown in the perfusion maps of Panels A and B. Panel D, perfusion maps and temporal fluctuations of the perfusion extracted from the regions $x$ and $y$ on the development day 10. The perfusion maps labeled with arterial and venous flow are calculated by averaging of a number of frames at minimum and maximum values of the perfusion at the region $y$, respectively. White arrows, the vein that provides a clear signal during the venous flow. Asterisk, the eye of the embryo. The exposure time for EDDs 3-4 is $10 \mathrm{~ms}$ while it is $5 \mathrm{~ms}$ for EDD 10. 


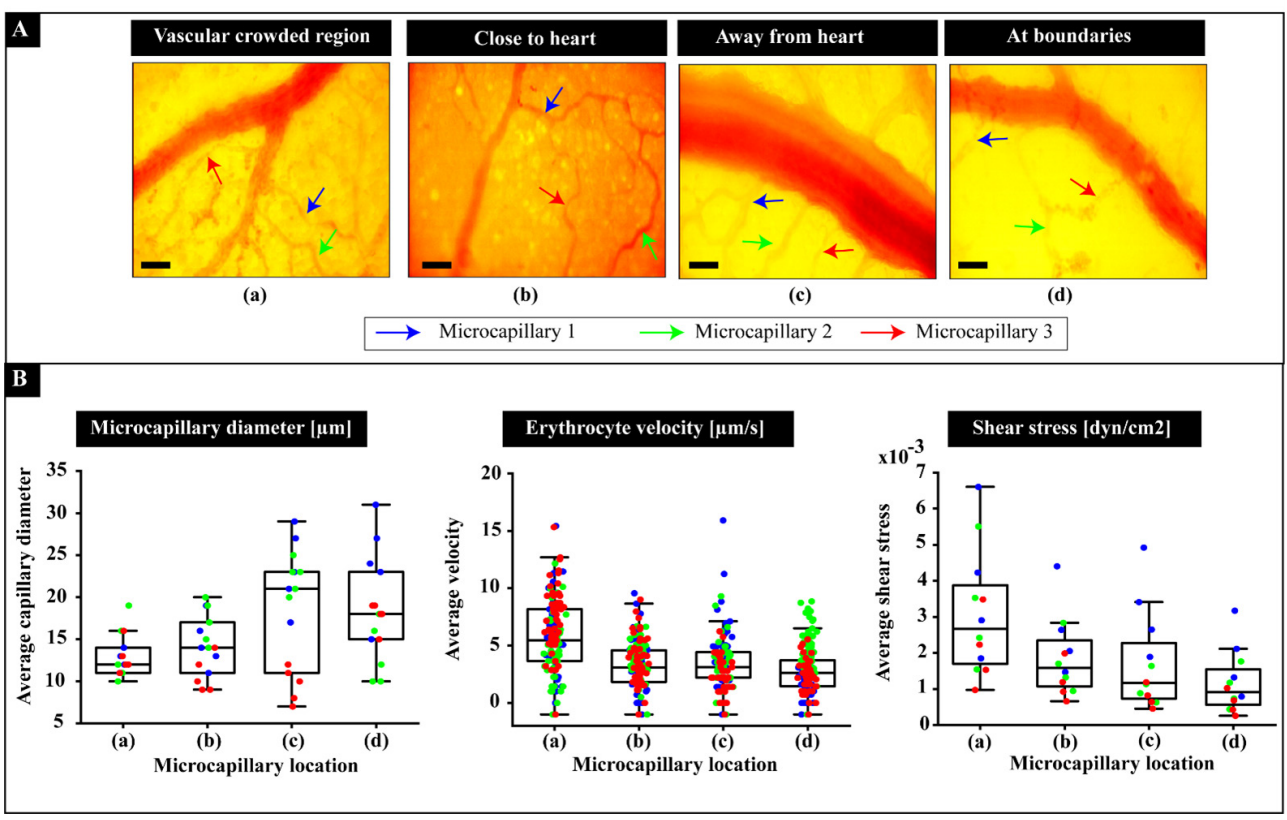

Fig. 6.6: Vessel diameter and erythrocytes velocity estimation within individual microcapillaries using SDF microscopy. Panel A shows the time-averaged snapshots of microcapillaries organization at multiple locations of chick vascular networks. scale bars, $100 \mu \mathrm{m}$. Three capillaries from each locations highlighted with colored arrows were selected for quantitative analysis. Panel B shows the quantitative analysis similar to Figure 6.2(B) were performed on the selected microcapillaries from different locations to approximate the diameter and erythrocytes velocities. These values were served as an input for computational modeling to estimate the shear stress. For the shear stress profile, erythrocyte movements and tracking see Supplementary Figure 6.10, Visualization 6.10 and Visualization 6.11, respectively.

Quantifications of microcapillary diameter and erythrocytes velocities are depicted in Figure 6.6 (B). This information is further used as input for computational fluid dynamics simulations in order to predict fluid flow shear stresses in the capillaries. The results show an increasing trend in the microcapillary diameters starting from the heart towards the boundary regions of the vascularized membrane whereas the erythrocyte velocity flowing through these microcapillaries decreases since they are moving away from the heart. Interestingly, this is corroborated by the shear stress graph, which decreases on the regions from the heart to the boundaries. This points to a understanding that there is a decrease in blood flow resistance when the blood is supplied from heart to the surrounding tissues whereas it is other way round when the blood is moving towards heart. Knowledge about shear stress is crucial for understanding the dynamics of the capillary network such as the formation of a new micro-vessel. 


\section{Proof-of-concept study: LSCI on biofabricated tissue constructs}

As described in Section 6.2.8, the perfusable biofabricated tissue constructs were created and LSCI were performed using multiple flow rates. For the demonstrator experiment, we made three perfusable tissue constructs namely (1) acellular gelatin hydrogel (control), (2) fibrin hydrogel with $\mathrm{C} 2 \mathrm{C} 12$ cells (Tissue 1) and (3) fibrin hydrogel with $C_{2} C_{12}$ and HUVECs (Tissue 2) as mentioned in Figure 6.7(A). The gelatin hydrogel was created for the initial demonstrator experiment. An image of the perfusable channel is shown in 6.12(A). The speckle contrast map for the flow rate of $28.5 \mu \mathrm{l} / \mathrm{min}$ is also shown in 6.12(B). The measured speckle contrast across the cross section of the channel for the case of no flow (Brownian motion) and the flow rate of $28.5 \mu \mathrm{l} / \mathrm{min}$ is depicted in $6.12(\mathrm{C})$. A plot of speckle contrast at the center of the channel versus the flow rate and the equivalent volumetric flux is depicted in 6.12(D). Here, we observe that increasing the flow rate, the measured speckle contrast is decreased. In this experiment, for the scattering ratio parameter $(\rho)$ equals to 0.85 , the estimated perfusion response to the set flow rate becomes linear. A plot of the estimated perfusion across the cross section of the channel for the case of no flow and the set flow rate of $28.5 \mu \mathrm{l} / \mathrm{min}$ is depicted in 6.12(E). Finally, the estimated perfusion versus the set flow rate and the corresponding volumetric flux is depicted in 6.12(F).

The representative image of a biofabricated channel and the corresponding confocal images for the case of $\mathrm{C} 2 \mathrm{C} 12$ and HUVECs is depicted in Figure 6.7(B). The temporally averaged perfusion map for the channel with $\mathrm{C} 2 \mathrm{C} 12$ cells with the set flow rate of $10 \mu \mathrm{l} / \mathrm{min}$ is illustrated in Figure 6.7(C). A temporal profile of the estimated perfusion at three locations, namely, inlet, middle and outlet for the set flow rate of $10 \mu \mathrm{l} / \mathrm{min}$ is shown in Figure 6.7(D). A comparison between the estimated perfusion at these three locations for the case of no flow and the set flow rates of 5 and 10 $\mu \mathrm{l} / \mathrm{min}$ is illustrated in Figure 6.7(E). Similar analysis is performed for the channel with $C_{2} C_{12}$ and HUVECs cells and the data is shown in Figure 6.7(F-H). A video presentation of the flow application can be seen in Visualization 6.12. To the best of our knowledge, this study is the first to show that laser speckle contrast imaging is compatible with living engineered tissue constructs. By coupling fluid flow parameters to the organization of vascular networks, this leads to a deeper understanding regarding the structural information and mechanical environment of these developing vascular networks, which is an important component in controlling vascular organization in biofabricated tissues.

\subsection{Summary}

An artificial eggshell system was developed which enables the development of chick embryos up to embryo development day 10 and offers the possibility of studying the complete vascular network formation and organization over time. The culture system 


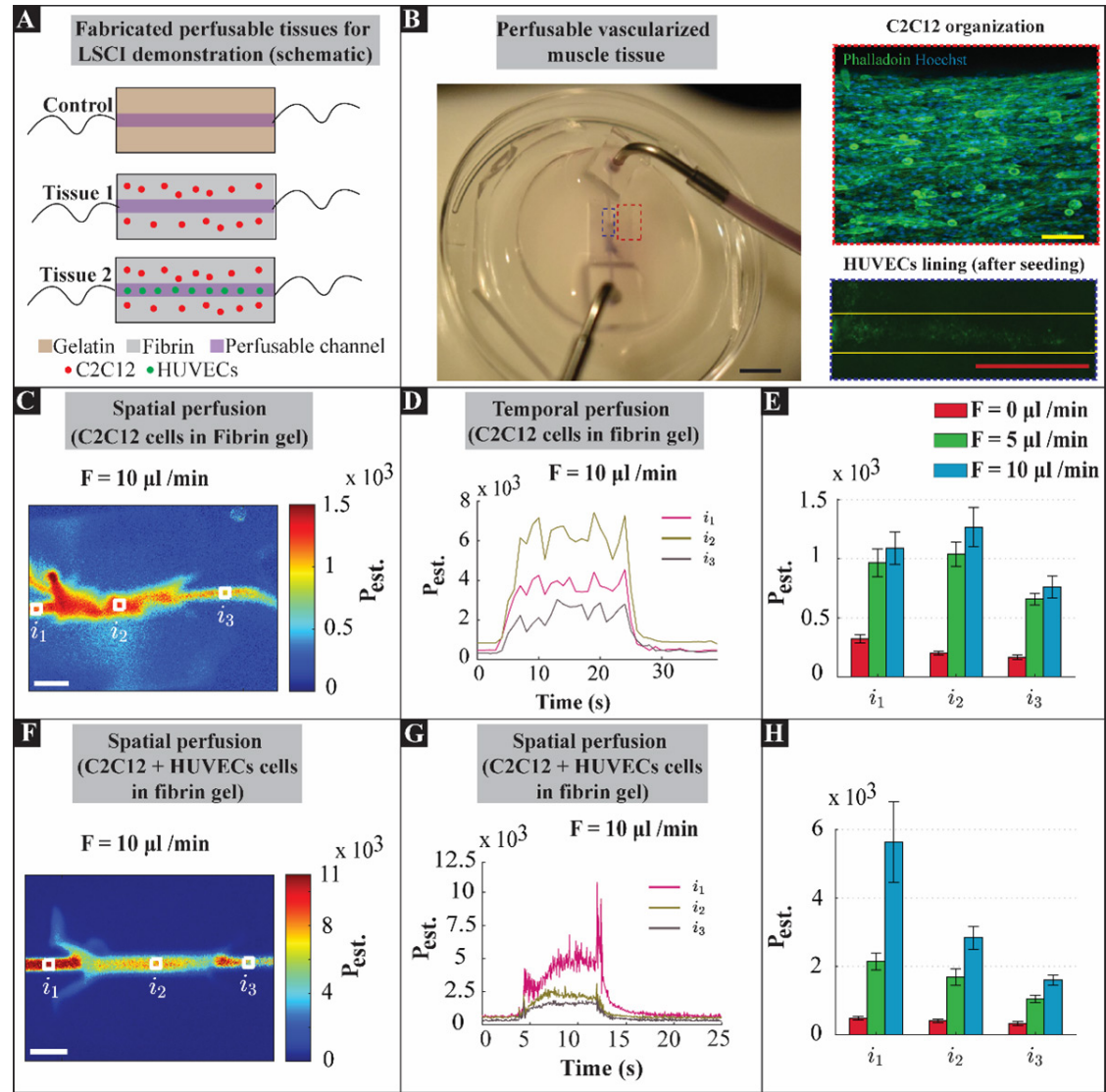

Fig. 6.7: LSCI on perfusable muscle tissues. Panel A shows the schematic figure of three perfusable hydrogel tissue construct namely acellular gelatin hydrogel (control), fibrin hydrogel with $\mathrm{C}_{2} \mathrm{C}_{12}$ cells (Tissue 1) and fibrin hydrogel with $\mathrm{C}_{2} \mathrm{C}_{12}+\mathrm{HUVEC}$ cells (Tissue 2). Schematic figure were created using Adobe Illustrator 2020. Data of gelatin hydrogel is illustrated in Supplementary Figure 6.12. Panel B, left, shows an image of the perfusable muscle tissue construct with Intralipid-dye perfusion setup. Top right, a confocal microscope image of the $\mathrm{C}_{2} \mathrm{C}_{12}$ cellular organization and densities within tissue construct, highlighted with the dashed red box in the left image. Bottom right, a fluorescence microscope image of the channel area highlighted with the dashed blue box in the left image. Panel $\mathbf{C}$ illustrates the temporally averaged perfusion map ( $\rho=0.85$ ) of the flow phantom with $\mathrm{C}_{2} \mathrm{C}_{12}$ cells with a flow rate of $F=10 \mu \mathrm{l} / \mathrm{min}$ for the case of tube on Delrin. scale bar, $1 \mathrm{~mm}$. Panel D illustrates the temporal profiles of the estimated perfusion $(F=10 \mu \mathrm{l} / \mathrm{min})$ associated with the white regions highlighted in Panel C. Panel E illustrates the estimated perfusion for various flow rates. Panel F depicts the temporally averaged perfusion map ( $\rho=0.85$ ) of the flow phantom with $\mathrm{C}_{2} \mathrm{C}_{12}$ and HUVECs cells with the flow rate of $F=10 \mu \mathrm{l} / \mathrm{min}$ for the case of tube on Delrin. scale bar, $1 \mathrm{~mm}$. Panel $\mathbf{G}$ depicts the temporal profiles of the estimated perfusion $(F=10 \mu \mathrm{l} / \mathrm{min})$ associated with white regions highlighted in Panel F. Panel H, depicts the estimated perfusion for various flow rates. Panels (C-D, F-G) are still images of Visualization 6.12. 
offers high accessibility as an area of $6 \mathrm{~cm}^{2}$ at the top of the system is open for optical probes. The culture system can accommodate $60 \mathrm{ml}$ of fertilized egg contents (egg contents is measured to vary from $52-56 \mathrm{ml}$ ) and offers the flexibility for long-term chick embryo culture (Figure 6.1). The secondary incubator (see Supplementary Figure 6.8) in which the samples were placed during imaging provided a relatively safe environment in terms of temperature and humidity control. On the other hand, we acknowledge that there are several challenges in the procedure of culturing samples and in the preparation for imaging, such as difficulties in cracking the eggshells at early development stage and transferring the egg content including the embryo into the artificial eggshell system.

In order to elucidate the relation between vascular structure and organization on the one hand and shear stresses and perfusion values on the other hand, which is beneficial information for the bioengineering community in order to understand and control vascular organization in engineered tissues, multiple imaging modalities were used. The color imaging during the development days revealed global structural changes of the vasculature such as vessel area, vessel length and branching density (Figure 6.2). The chick CAM vascular network includes large vessels and smaller capillaries that constantly change their diameter due to the remodeling mechanism [4]. Moreover, the fluid properties of fertilized egg contents such as thin/thick albumin and egg yolk change during the course of development. As this will result in a variation of the LSCI scattering profiles, we performed LSCI of flow phantoms consisting of microtubing of various diameters mounted on the light scattering and absorbing backgrounds. Dependency of the measured speckle contrast on both tube diameter and scattering/absorbing background was observed. A model that takes into account the scattering ratio was used to estimate perfusion. It is shown that with knowledge about the tube diameter and scattering level of the background, perfusion values can be estimated that are proportional to the actual volumetric flux or flow rate (Figure 6.3). However, there are three reasons that prevent us to directly correlate flow phantom results to absolute blood flow values in the developing chick vasculature. (1) Although a blood mimicking fluid was pumped through microtubing in flow phantom experiments, it is different from the blood in terms of particle size and viscosity. (2) The scattering and absorbing backgrounds used in the flow phantom study were static and simplistic while the CAM model is a dynamic environment with its scattering and absorbing properties changing over developing stages. (3) In order to make the estimated perfusion values independent of tube diameters, vessel diameters in the CAM model must be accurately measured. Since there are blood vessels with various diameters at each development stage of the CAM model, blood vessel segmentation and characterization may be an intensive machine vision task that is out of the scope of this current research.

To illustrate that LSCI is not only compatible with developing chick embryos but 
also with bioengineered tissues, perfusable muscle tissue constructs were prepared using $C_{2} C_{12}$ mouse myoblast cells and GFP-HUVEC endothelial cells (Figure 6.7). Even though the presented construct only contains a simple straight perfused channel, this study shows that LSCI imaging is compatible with opaque tissue constructs, thus highlighting the potential for the bioengineering community.

The tracking of spatiotemporal fluctuations of blood flow within vasculature of varying complexity and constantly changing vessel diameter, is important for validating the physiological significance of mechanical signals developed within the vascular networks. In this work, LSCI perfusion maps of the entire chick CAM vasculature within days 3-6 were obtained and the estimated perfusion levels on three locations of a vessel were compared over the development days (Figure 6.4). It is known that distinct arteries and veins are formed within hours after the on-set of the heart beat in flow driven chick CAM vasculature [4] . Earlier studies have shown that veins can transform into arteries when subjected to high shear stress forces by flow manipulation, proving that arterial-venous differentiation is a flow-driven highly dynamic process [4]. To our knowledge, this study is the first to show the blood perfusion within artery and veins separately over space and time at the developmental days 3-4 and 10 (Figure 6.5).

In this study, SDF microscopy was performed in order to examine erythrocytes velocities in vessels that vary in size. With the analysis of the SDF data, microcapillary diameters and erythrocyte velocities were quantified. Then, with the combined data of velocity and diameter, fluid flow shear stresses for the microcapillaries of varying diameters were evaluated using computational modeling (Figure 6.6(B)). This direct knowledge about shear stress is crucial for tissue engineers when perfusing biofabricated vascularized tissues with the goal of tuning the vascular organization, as fluid flow shear stress appears to be the main fluid flow related parameter that controls vascular organization $[1,2]$.

As a concluding step in this study, biofabricated tissue constructs were imaged with LSCI to investigate their ability to be perfused (Figure 6.7). Knowing the localized flow induced shear stress values and associated spatiotemporal information will help bioengineers to fine-tune the vascular organization using mechanical signals. We show that information about spatiotemporal perfusion can be obtained by LSCI in a non-invasive manner, using an affordable experimental setup and with a reasonable post-processing time. Transparent bioengineered tissues are of interest to the tissue engineers. However, engineered tissues often becomes opaque because of cells maturation over time. Moreover, it is difficult to visualize perfusion even using fluorescent beads within these tissues. Recent studies show that intact-tissue imaging requires complicated staining procedure and expensive experimental setup for visualizing the structural and mechanical information [42]. These tissue constructs we made out of gelatin and fibrin, which are commonly used hydrogel polymers, provided us with 
different levels of opacity, that are used to explore the LSCI application on engineered tissues. Since it was not possible to make a perfusable channel within acellular, less stiff fibrin hydrogel, we used gelatin hydrogel as a control. The addition of HUVECs to the fibrin hydrogel constructs decreases the vascular permeability which is visible during perfusion (see Visualization 6.12). To our knowledge, this study is the first to show that LSCI is compatible with engineered tissues and even to study HUVEC cells barrier properties.

\subsection{Conclusion and future work}

In conclusion, we employed multimode imaging schemes, namely color imaging, LSCI and SDF microscopy, for studying the vascular organization and their associated flow dynamics in a well-known CAM model. Additionally, the compatibility of LSCI with perfusable bioengineered muscle tissue constructs was shown. This resulted in exploring the flow dynamics and estimation of shear stress within the vasculature of varying complexity. Even though the culture system is simple, it is compatible with the aforementioned imaging modalities in terms of providing access to a wide vasculature surface and offering the possibility of placing the probe to multiple locations of the vascular network. Future work will focus on perturbing the developing chick embryo using external localized fluid flows and multiple growth factors. Additionally, the chick embryo culture system as well as the perfusable engineered muscle tissue model will be improved to further elucidate the role that fluid flow related mechanical signals can play in the tuning of vascular organization.

\section{Acknowledgments}

The authors acknowledge the financial support from the ERC consolidator grant (724469) of J.R. and Open Technology program of the Netherlands Organization for Scientific Research (NWO), Domain Applied and Engineering Sciences, under grant number 14538 of W.S. The authors thank Design Lab of the University of Twente for laser cutting PMMA blocks, used to make the PDMS culture system. Illustrations of the manuscript were partially created with Biorender.com. The authors thank Deepti Rana for sharing hydrogel materials.

\section{Author contribution statement}

P.P., A.C. designed and performed the experiments. P.P. wrote the initial draft. A.C. assisted with incubator modification for PDMS based ex-ovo culture experiments and performed multi-mode imaging. P.P. performed fluid dynamics simulations. P.P. and 
V.T. fabricated the engineered tissue perfusion chambers. T.K. helped with assembly of imaging setup. J.Z. performed cell culture studies and confocal microscopy. A.C. and P.P., interpreted and analyzed the data. J.R. and W.S. conceived and coordinated the research. All authors contributed to a critical discussion of the data and participated in writing the manuscript.

\section{Ethics statement}

According to the Dutch animal care guidelines, IACUC approval for chicken embryo experimentation is not necessary unless hatching is expected. Moreover, only experiments with chick embryos of development EDD14 and older need IACUC approval. The embryos used in this study were all in early stages of embryo development (between EDD3 and EDD10).Fertilized chicken eggs used in this study were purchased from approved poultry egg farms in Netherlands. GFP-HUVECs culture studies were approved by Dutch Ministry and experiments were performed under ML-1 laboratory environment, followed by the university policies and standard protocol.

\section{Data availability}

The datasets generated during and/or analyzed during this study to reproduce Fig. 6.1(A), top; Fig. 6.1(C); Fig. 6.2 raw RGB images; Fig. 6.3(C-H); Figs. 6.4-6.5; Fig. 6.6(A); Fig. 6.7(C-H); Supplementary Fig. 6.11 (S4) and Supplementary Fig. 6.12(B-F) (S5) are available in the following Figshare repository:

https://doi.org/10.6084/m9.figshare.16402662.

The rest of the datasets generated during and/or analyzed during this study to reproduce Fig. 6.2(B); Fig. 6.6(B) and Supplementary Figs. 6.9-6.10 (S2-S3) are available from the corresponding author on reasonable request. 


\subsection{Supplementary figures}

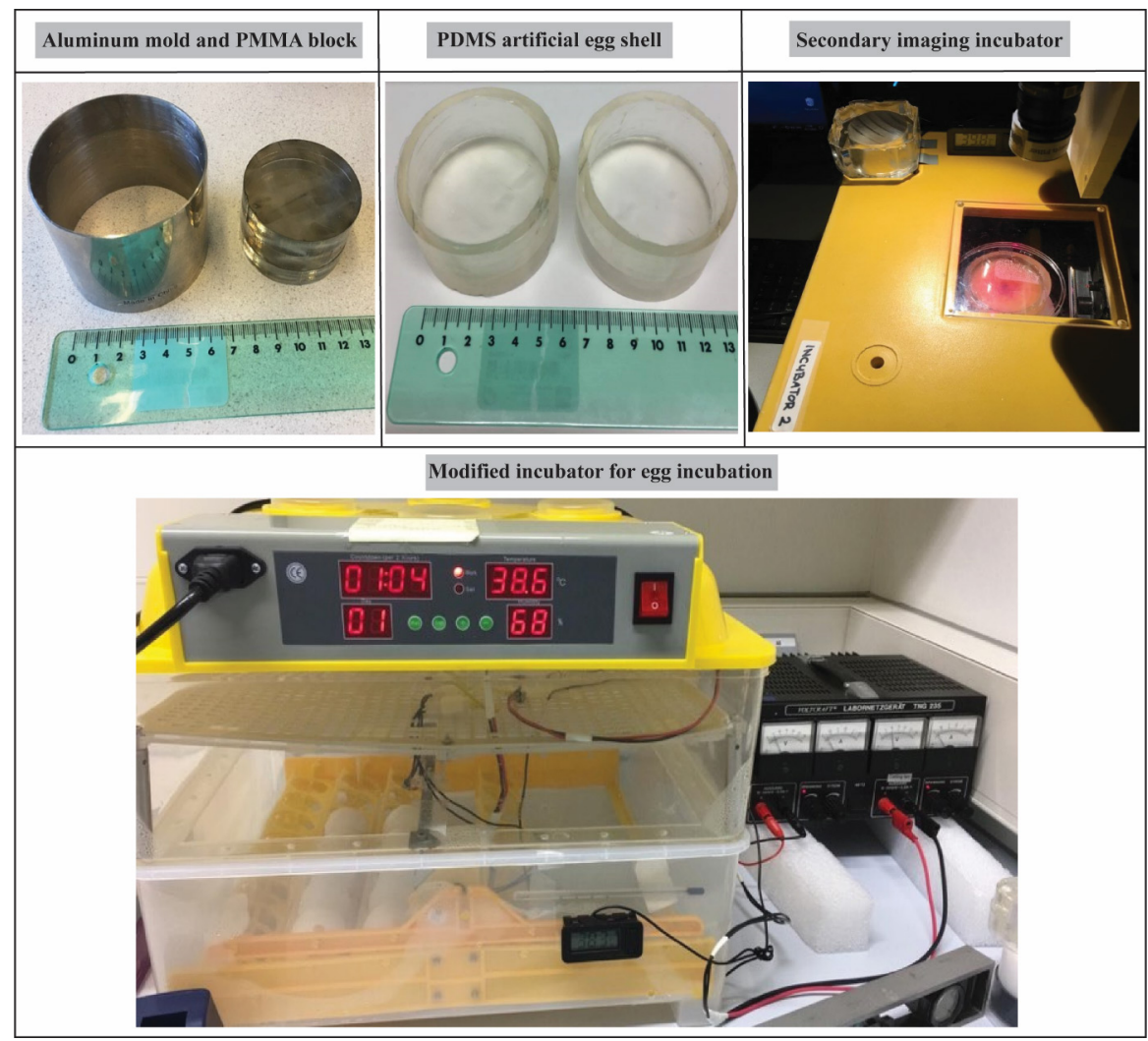

Fig. 6.8: Artificial eggshell fabrication and fertilized chicken eggs incubation. Top panels (left and middle) show the aluminum mold and circular shaped laser-cut PMMA block, used for PDMS casting to make the artificial eggshell, where fertilized chicken embryos are cultured. Top panel (right), secondary incubator with artificial eggshell cultured chick embryo sample used for multimode imaging experiments. Bottom panel, shows the modified egg incubator with controllable fan and additional temperature sensors. Fertilized chicken eggs and artificial eggshell cultured chick embryo samples were incubated in this incubator throughout the study. Only during the imaging, artificial eggshell cultured chick embryo samples were transferred to the secondary imaging incubator. 


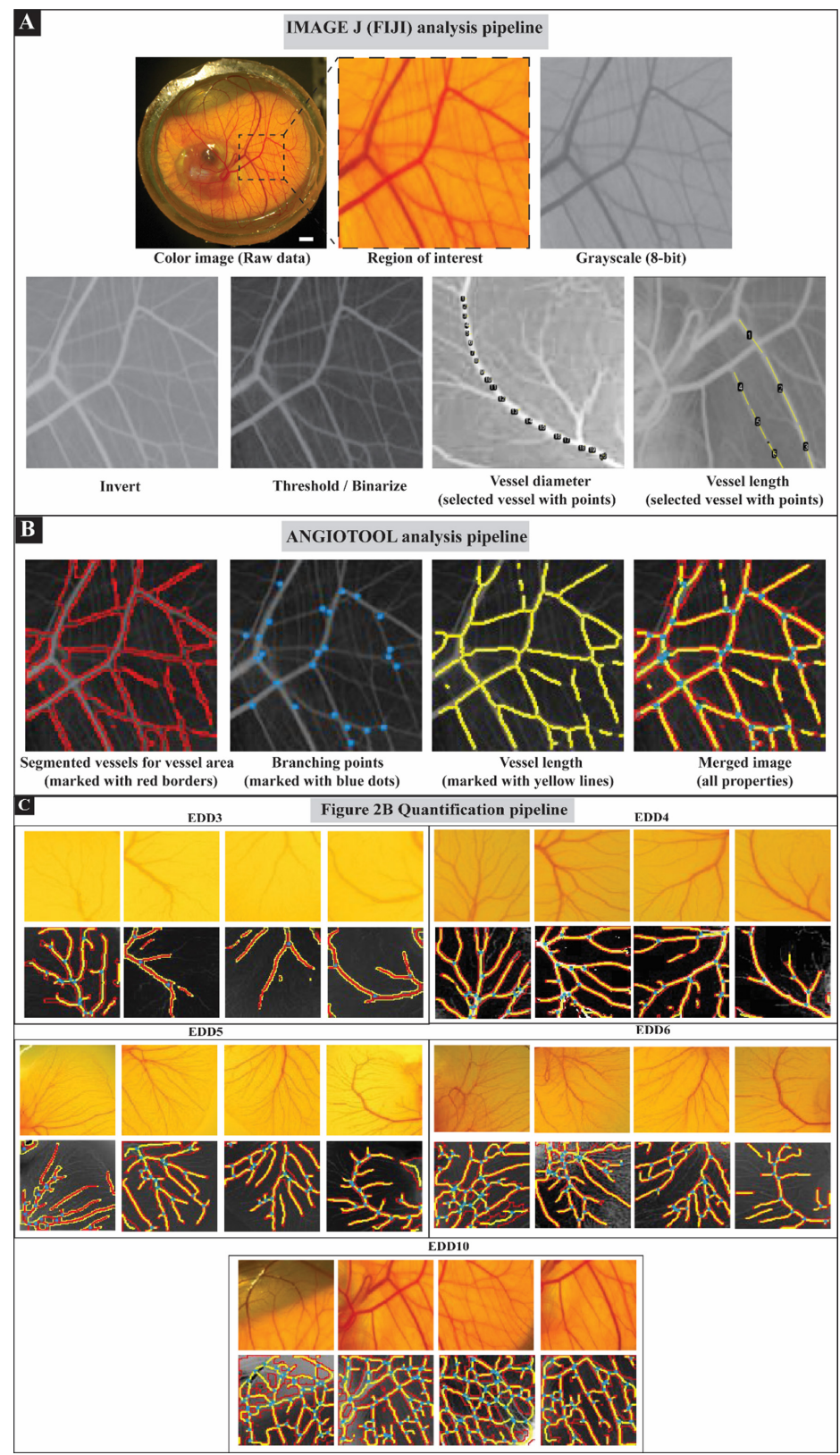

Fig. 6.9: Complementary data for quantitative image analysis of vascular networks. Panel A shows the steps involved in binarizing of RAW data using ImageJ (FIJI) software tool for vessel diameter and length measurement. Panel B shows the steps involved in Angiotool Plugin, used for calculating vascular network properties such as vessel length, vessel diameter, branching points and lacunarity. Panel C shows the colored raw data of selected ROIs and respective merged images of vessel properties obtained from Angiotool Plugin. These measured values are used for plotting graphs in Figure 6.2(B). 

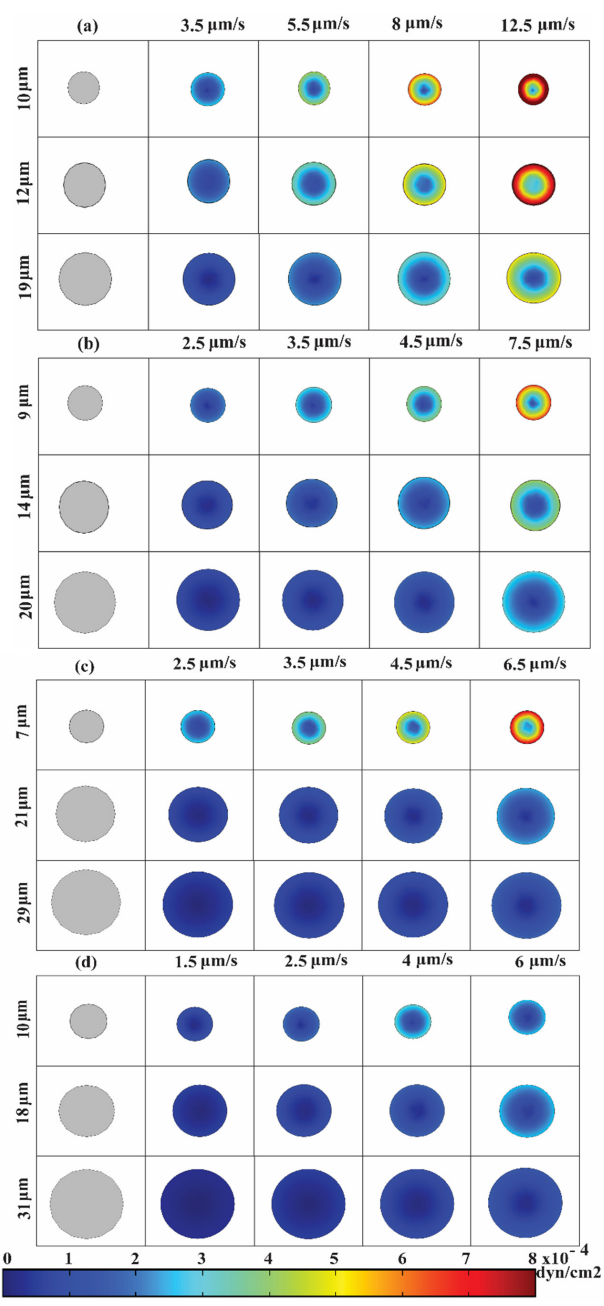

Fig. 6.10: Computational models showing the average shear stress values within varying microcapillary diameters obtained from SDF microscopy data. Panels (a-d) show the cross-sectional view of selected microcapillary diameters from SDF microscopy data, which are highlighted in Figure 6.6(A). By coupling the microcapillary diameters and erythrocyte velocities from SDF microscopy data, the average volumetric shear stresses are computed for different velocities using COMSOL Multiphysics software Version 5.5 as shown in the panels (a-d). Three microcapillary from four different regions are selected for the diameter estimation and erythrocyte tracking. Selected capillaries are highlighted in blue, green and red arrows (refer Figure 6.6(A)). Min, Mid and Max values of microcapillary diameters are taken from Figure 6.6(B) (left graph), used as structural input geometry for the computational models. 25, $50,75 \%$ and max values of erythrocyte velocities are taken from Figure 6.6(B) (middle graph), as input velocities. In total combination of 12 microcapillary geometries with varying diameters and 16 different input velocities were tested. Refer Figure 6.6(B) for average shear stress graph. See Visualization 6.10 and Visualization 6.11 for erythrocyte movements and tracking, respectively. 


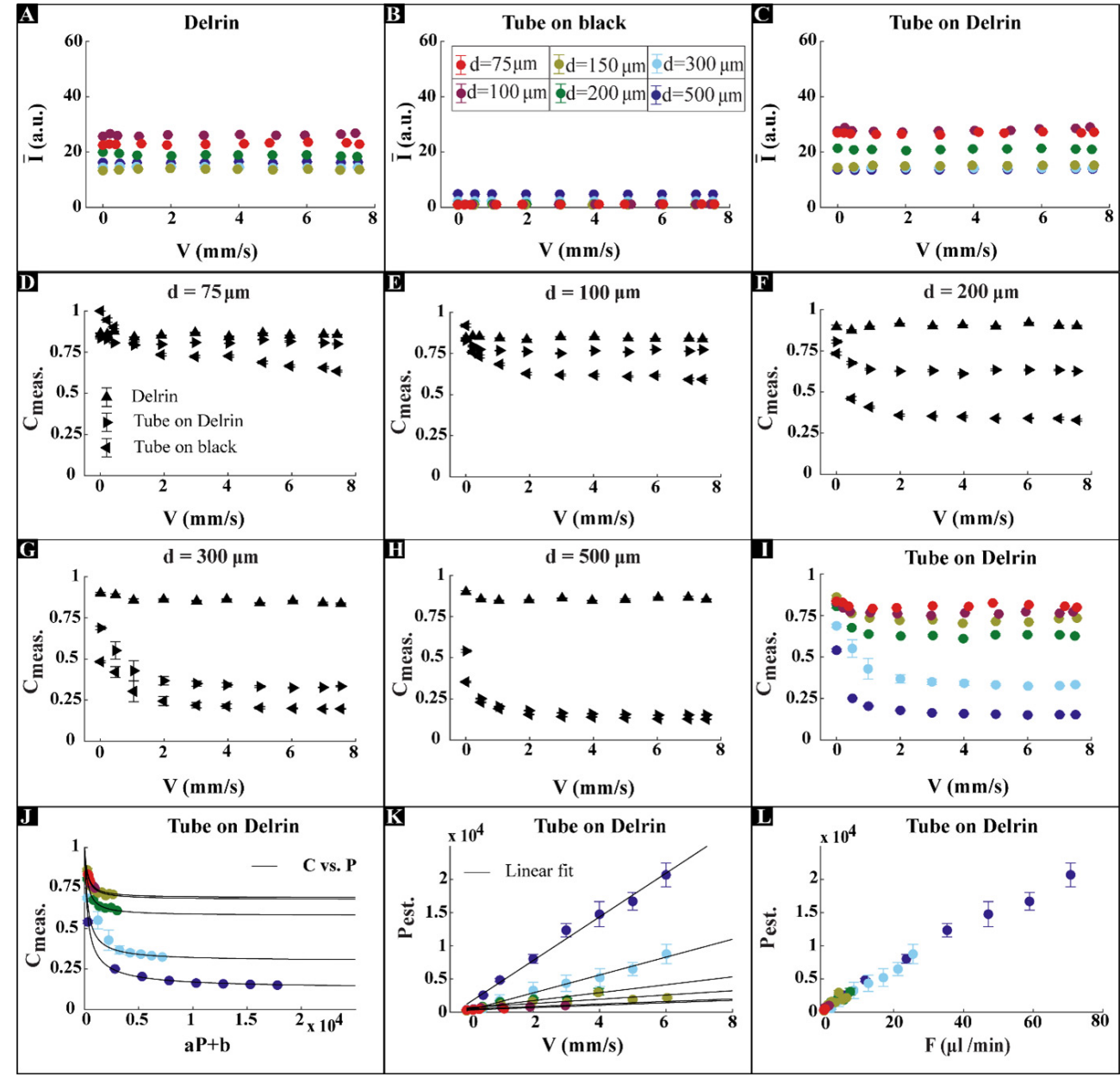

Fig. 6.11: Complementary data of LSCI for microtubing flow phantoms. Panels A-C, mean intensity versus the volumetric flux for Delrin, tube on black and tube on Delrin, respectively. Data points are mean \pm standard deviation. The colored data points in Panels A-C, I-L correspond to various tube diameters which are defined in Panel B. Panels D-H, measured speckle contrast versus the volumetric flux as a comparison of Delrin, tube on Delrin and tube on black for tubing diameters of 75, 100, 200, 300 and $500 \mu \mathrm{m}$, respectively. Panels I-L correspond to the case of tube on Delrin. Panel I, measured speckle contrast versus the volumetric flux. Panel J, measured speckle contrast versus the shifted and scaled versions of perfusion overlapped with theoretical relation between the speckle contrast and perfusion. Panel K, estimated perfusion versus the applied volumetric flux with a linear fit for each tube diameter. Panel L, estimated perfusion versus the flow rate. 


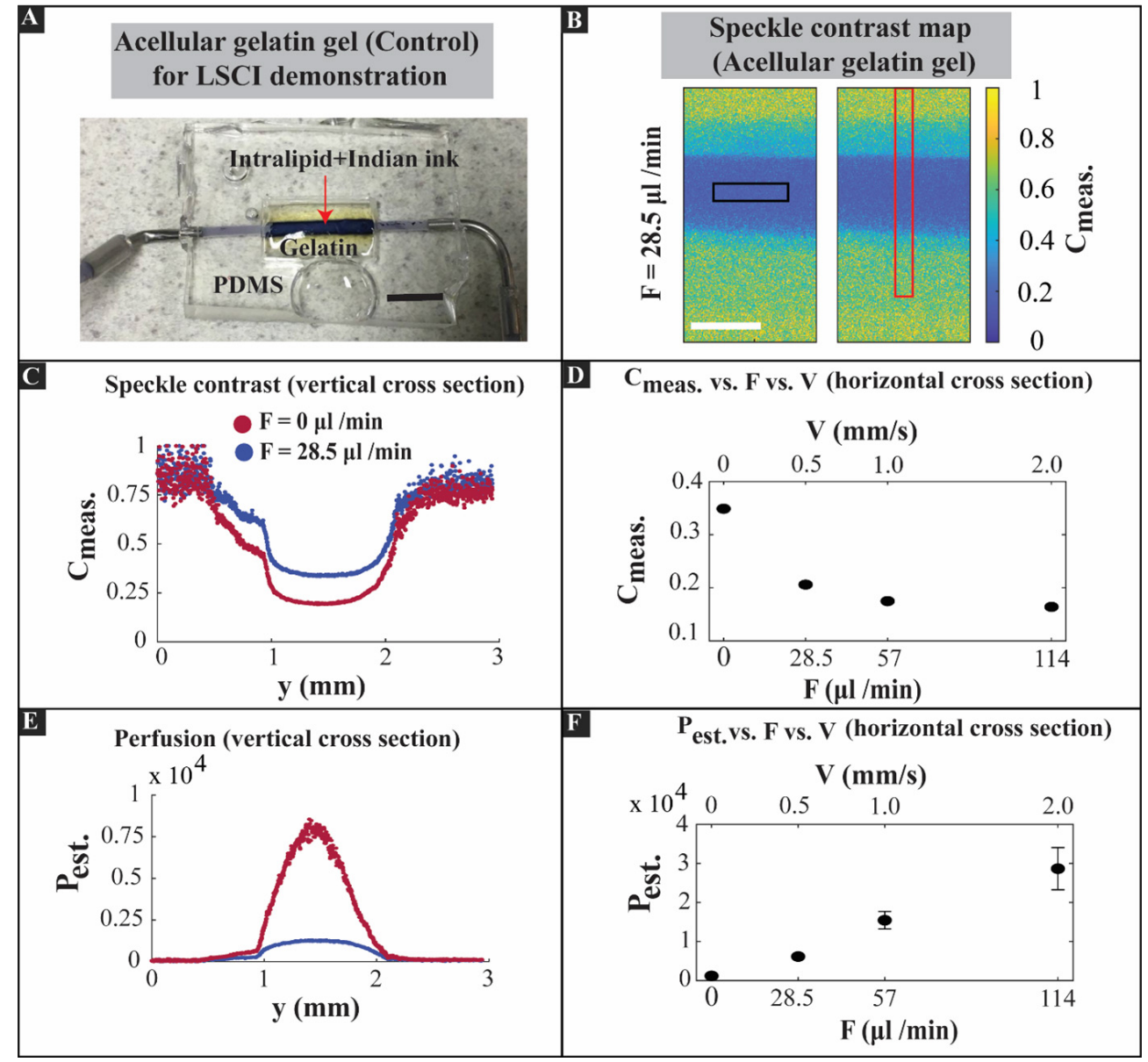

Fig. 6.12: Complementary data of LSCI flow phantom on gelatin hydrogel. Panel A shows the perfusable gelatin hydrogel construct without cells. Black scale bar, $5 \mathrm{~mm}$. Gelatin hydrogel was used for initial demonstrator testing and it also served as control and substitute to plain fibrin hydrogel construct. Panel B shows the spatial speckle contrast map for the case of tube on black. White scale bar, $1 \mathrm{~mm}$. Panel C shows speckle contrast over the cross sectional vertical region (highlighted with red box) indicated in Panel B. Panel D shows speckle contrast versus flow rate and volumetric flux calculated from the horizontal region (highlighted with black box) indicated in Panel B. Panel E depicts the estimated perfusion ( $\rho=0.85$ ) associated with the speckle contrast shown in Panel C. Panel F depicts the estimated perfusion $(\rho=0.85)$ associated with the speckle contrast shown in Panel D. 


\section{References}

[1] J. L. Lucitti, E. A. Jones, C. Huang, J. Chen, S. E. Fraser, and M. E. Dickinson, "Vascular remodeling of the mouse yolk sac requires hemodynamic force", Development 134, 3317-3326 (2007).

[2] H. Nakajima and N. Mochizuki, "Flow pattern-dependent endothelial cell responses through transcriptional regulation”, Cell Cycle 16, 1893-1901 (2017).

[3] H. U. Wang, Z.-F. Chen, and D. J. Anderson, "Molecular distinction and angiogenic interaction between embryonic arteries and veins revealed by ephrin-b2 and its receptor eph-b4", Cell 93, 741-753 (1998).

[4] F. Le Noble, D. Moyon, L. Pardanaud, L. Yuan, V. Djonov, R. Matthijsen, C. Bréant, V. Fleury, and A. Eichmann, "Flow regulates arterial-venous differentiation in the chick embryo yolk sac", Development 131, 361-375 (2004).

[5] T.-H. Nguyen, A. Eichmann, F. Le Noble, and V. Fleury, "Dynamics of vascular branching morphogenesis: the effect of blood and tissue flow", Physical Review E 73, 061907 (2006).

[6] P. A. Galie, D.-H. T. Nguyen, C. K. Choi, D. M. Cohen, P. A. Janmey, and C. S. Chen, "Fluid shear stress threshold regulates angiogenic sprouting", Proceedings of the National Academy of Sciences 111, 7968-7973 (2014).

[7] J. W. Song and L. L. Munn, "Fluid forces control endothelial sprouting", Proceedings of the National Academy of Sciences 108, 15342-15347 (2011).

[8] S. Kim, H. Lee, M. Chung, and N. L. Jeon, "Engineering of functional, perfusable 3d microvascular networks on a chip", Lab on a Chip 13, 1489-1500 (2013).

[9] J. G. DeStefano, A. Williams, A. Wnorowski, N. Yimam, P. C. Searson, and A. D. Wong, "Real-time quantification of endothelial response to shear stress and vascular modulators", Integrative Biology 9, 362-374 (2017).

[10] J. Seo, D. Conegliano, M. Farrell, M. Cho, X. Ding, T. Seykora, D. Qing, N. S. Mangalmurti, and D. Huh, "A microengineered model of rbc transfusion-induced pulmonary vascular injury”, Scientific reports 7, 1-10 (2017).

[11] M. N. Chávez, G. Aedo, F. A. Fierro, M. L. Allende, and J. T. Egaña, "Zebrafish as an emerging model organism to study angiogenesis in development and regeneration", Frontiers in physiology 7, 56 (2016).

[12] N. Bahrami and S. J. Childs, "Development of vascular regulation in the zebrafish embryo", Development 147 (2020).

[13] A. V. Gore, K. Monzo, Y. R. Cha, W. Pan, and B. M. Weinstein, "Vascular development in the zebrafish", Cold Spring Harbor perspectives in medicine 2, a006684 (2012). 
[14] A. L. Seynhaeve and T. L. Ten Hagen, "Intravital microscopy of tumor-associated vasculature using advanced dorsal skinfold window chambers on transgenic fluorescent mice", Journal of visualized experiments: JoVE (2018).

[15] M. Alieva, L. Ritsma, R. J. Giedt, R. Weissleder, and J. Van Rheenen, "Imaging windows for long-term intravital imaging: General overview and technical insights", Intravital 3, e29917 (2014).

[16] K. van Doorn and J. G. Sivak, "Blood flow dynamics in the snake spectacle", Journal of Experimental Biology 216, 4190-4195 (2013).

[17] Y. Matsubara, A. Kuroiwa, and T. Suzuki, "Efficient harvesting methods for early-stage snake and turtle embryos", Development, growth \& differentiation 58, 241-249 (2016).

[18] P. Nowak-Sliwinska, T. Segura, and M. L. Iruela-Arispe, "The chicken chorioallantoic membrane model in biology, medicine and bioengineering", Angiogenesis 17, 779-804 (2014).

[19] W. Huang, M. Itayama, F. Arai, K. S. Furukawa, T. Ushida, and T. Kawahara, “An angiogenesis platform using a cubic artificial eggshell with patterned blood vessels on chicken chorioallantoic membrane", PLoS One 12, e0175595 (2017).

[20] A. Sharma, N. Ishak, T. Swee-Hin, and M. Pramanik, "High resolution, label-free photoacoustic imaging of live chicken embryo developing in bioengineered eggshell", Journal of biophotonics 13, e201960108 (2020).

[21] N. Ishak, M. Chan, S. C. Ang, C. Cheung, and S.-H. Teoh, "Bioengineered threedimensional transparent eggshell as a chicken embryo experimentation platform for biomedical research", Engineering Reports 2, e12092 (2020).

[22] J. M. Schmitt, "Optical coherence tomography (oct): a review", IEEE Journal of selected topics in quantum electronics 5, 1205-1215 (1999).

[23] M. Xu and L. V. Wang, "Photoacoustic imaging in biomedicine", Review of scientific instruments 77, 041101 (2006).

[24] M. R. Lowerison, C. Huang, F. Lucien, S. Chen, and P. Song, "Ultrasound localization microscopy of renal tumor xenografts in chicken embryo is correlated to hypoxia", Scientific reports 10, 1-13 (2020).

[25] R. T. Sadikot and T. S. Blackwell, "Bioluminescence imaging", Proceedings of the American Thoracic Society 2, 537-540 (2005).

[26] D. A. Boas and A. K. Dunn, "Laser speckle contrast imaging in biomedical optics", Journal of biomedical optics 15, 011109 (2010).

[27] A. Nadort, K. Kalkman, T. G. Van Leeuwen, and D. J. Faber, "Quantitative blood flow velocity imaging using laser speckle flowmetry", Scientific reports 6, 1-10 (2016). 
[28] E. Du, S. Shen, S. P. Chong, and N. Chen, "Multifunctional laser speckle imaging", Biomedical optics express 11, 2007-2016 (2020).

[29] P. Goedhart, M. Khalilzada, R. Bezemer, J. Merza, and C. Ince, "Sidestream dark field (sdf) imaging: a novel stroboscopic led ring-based imaging modality for clinical assessment of the microcirculation.", Optics express 15, 15101-15114 (2007).

[30] J. W. Goodman, Speckle phenomena in optics: theory and applications (Roberts and Company Publishers) (2007).

[31] A. Fercher and J. D. Briers, "Flow visualization by means of single-exposure speckle photography", Optics communications 37, 326-330 (1981).

[32] D. D. Duncan and S. J. Kirkpatrick, "Can laser speckle flowmetry be made a quantitative tool?”, JOSA A 25, 2088-2094 (2008).

[33] J. D. Briers and S. Webster, "Laser speckle contrast analysis (lasca): a nonscanning, full-field technique for monitoring capillary blood flow", Journal of biomedical optics 1, 174-179 (1996).

[34] K. Christensen-Jeffries, J. Brown, P. Aljabar, M. Tang, C. Dunsby, and R. J. Eckersley, "3-d in vitro acoustic super-resolution and super-resolved velocity mapping using microbubbles", IEEE transactions on ultrasonics, ferroelectrics, and frequency control 64, 1478-1486 (2017).

[35] M. L. Ommen, M. Schou, C. Beers, J. A. Jensen, N. B. Larsen, and E. V. Thomsen, "3d printed calibration micro-phantoms for super-resolution ultrasound imaging validation", Ultrasonics 114, 106353 (2021).

[36] R. Michels, F. Foschum, and A. Kienle, "Optical properties of fat emulsions", Optics express 16, 5907-5925 (2008).

[37] M. C. Meinke, G. J. Müller, J. Helfmann, and M. Friebel, "Optical properties of platelets and blood plasma and their influence on the optical behavior of whole blood in the visible to near infrared wavelength range", Journal of Biomedical Optics 12, 014024 (2007).

[38] E. Zudaire, L. Gambardella, C. Kurcz, and S. Vermeren, "A computational tool for quantitative analysis of vascular networks", PloS one 6, e27385 (2011).

[39] H.-S. Ji, J.-Y. Lee, and S.-J. Lee, "In-vitro study on the hemorheological characteristics of chicken blood in microcirculation", Korea-Australia Rheology Journal 19, 89-95 (2007).

[40] J. Abbiss, T. Chubb, and E. Pike, "Laser doppler anemometry", Optics \& Laser Technology 6, 249-261 (1974).

[41] X. Liu, J. Wei, L. Meng, W. Cheng, X. Zhu, J. Lu, and P. Li, "Motion correction of laser speckle imaging of blood flow by simultaneous imaging of tissue structure and non-rigid registration”, Optics and Lasers in Engineering 140, 106526 (2021). 
[42] K. Chung, J. Wallace, S.-Y. Kim, S. Kalyanasundaram, A. S. Andalman, T. J. Davidson, J. J. Mirzabekov, K. A. Zalocusky, J. Mattis, A. K. Denisin, et al., "Structural and molecular interrogation of intact biological systems", Nature 497, 332-337 (2013). 


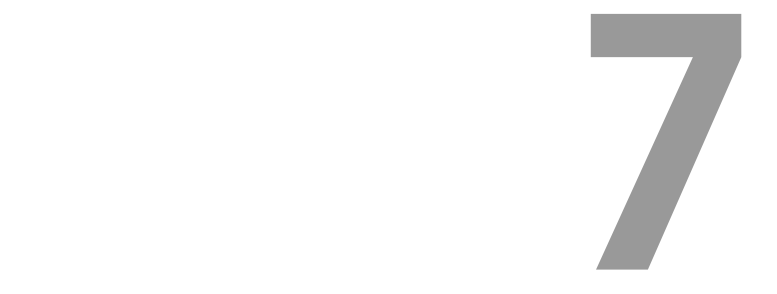

\section{Conclusion and outlook}

\subsection{Concluding marks}

Throughout this thesis, we made a closer look at the challenge of movement artefacts in a handheld LSCI system.

In Chapter 2, we concluded that the magnitude of movement artefacts depends on the optical properties of the medium. We explicitly showed that movement artefacts increase with decreasing scattering coefficient and based on our explanation it is to be expected that movement artefacts will also increase with decreasing absorption. We concluded that in the typical handheld motions (1) translation of the beam on the surface of the medium plays a dominant role over wavefront tilting in decreasing speckle contrast due to motion, and (2) the dominance of translation over tilt tends to decrease as the medium becomes less scattering and likely less absorbing. This means that in a handheld LSCI measurement, movement artefact correction based on a simple look-up table approach is not feasible without knowledge of the applied translations and tilts and also optical properties of the tissue.

Nowadays, most of the laser speckle perfusion imaging systems use engineered diffusers because it is an easy method for creating uniform illumination. In Chapter 3 , we have shown that using engineered diffusers is the worst option in terms of movement artefacts, with planar wavefronts being the best option. However, creating a planar beam of large size requires bulky optics. Furthermore, planar and spherical waves make a non-uniform Gaussian-shape light intensity which might affect measurement of speckle contrast at ROIs a couple of centimeters away from the beam center due to a lower average intensity. 
A model has been developed in Chapter 4 with the purpose of predicting movement artefacts caused by translation in handheld laser speckle contrast perfusion imaging observed in Chapter 3. Our model is based on the optical Doppler shift distributions associated with the range of wave vectors for illumination and detection. It is shown that the speckle contrast drop which is a measure of movement artefacts depends on the applied translational velocity, the type of illumination, and the geometry of detection. In the model, a connection has been established between the contrast measured in a typical LSCI and the optical Doppler shifts. What has been investigated in this chapter includes the combination of (1) finite scattering level and the plane wave illumination and (2) high scattering level and illumination with plane waves, spherical waves and scrambled waves.

We have examined handheld LSCI in a clinical research setting in Chapter 5 by introducing a post-processing procedure and made a comparison with mounted measurements. Our results showed that handheld measurements are reliable in terms of visual similarity compared to mounted measurement. The compact and portable HAPI probe realizes a comfortable skin perfusion measurement for both patients and medical staff. The performance of background correction reduced average perfusion differences between handheld and mounted measurements to $16.4 \pm 9.3 \%$ (mean \pm std, $n=11$ ), median of $23.8 \%$. Depending on the application in a clinical practice, this difference induced by movement artefacts may be acceptable.

Lastly, we employed multimodal imaging, namely color imaging, LSCI and SDF microscopy, for studying the vascular organization and their associated flow dynamics in a well-known CAM model in Chapter 6. The compatibility of LSCI with perfusable bioengineered muscle tissue constructs was shown. This resulted in exploring the flow dynamics and estimation of shear stress within the vasculature of varying complexity. Even though the culture system is simple, it is compatible with the aforementioned imaging modalities in terms of providing access to a wide vasculature surface and offering the possibility of placing the probe to multiple locations of the vascular network. Future work will focus on perturbing the developing chick embryo using external localized fluid flows and multiple growth factors.

\subsection{Future directions}

In an in-vivo LSCI measurement, the subject of interest is human skin which is of finite scattering level. It is essential to combine the developed model introduced in Chapter 4 with a Monte-Carlo simulation of light propagation in order to calculate the wave vector distribution of detected light and use this as input to our theory in chapter 4 to predict the speckle contrast drop. This will cover the case of scrambled or spherical beam illumination on a static medium with a finite scattering level. In addition to the validation of the theoretical and analytical curves of time-integrated 
speckle contrast versus the applied translational speed using simulation of dynamic speckles that we performed in chapter 4 , the theory has been experimentally validated by us. Due to the time limitation, the experimental results are not included in Chapter 4. Also, the model will be expanded to explain a dynamic medium with unordered motions of the scatterers.

Our current handheld LSCI system is still connected to a console with a computer and a laser power supply using a quite thick cable. This wired implementation will form an obstacle to clinical introduction in many settings. A wired system imposes limitations if measurements are to be performed on body parts such as the lower extremities. It would be strongly preferable if the LSCI operator can move around freely with the device. Therefore, we are currently developing a wireless version of the handheld perfusion imager. In the near future, we foresee application of this device in the framework of reconstructive surgery as well as burn wound imaging in a clinical research setting.

In long term, if we look at ten years from now, it is envisioned that the next generation of LSCI systems will have the possibility of operating both in mounted and handheld modes. Wireless LSCI systems will be used in operating theaters. With an optimal choice of the laser source, their total cost will reduce so a wide range of medical centers can afford them. At the moment, the features of the LSCI systems are limited to showing perfusion maps and color images, while the upcoming devices will contribute to image segmentation, interpretation and diagnosis. This will help clinicians to perform better treatment of skin diseases, and to reduce the number of repeat surgeries in reconstructive surgery, to mention a few applications. For each of these applications, systematic research will learn whether or not the movement artefacts during handheld operation using spherical or planar wavefronts will be sufficiently low to have no detrimental effect on the medical conclusions drawn from perfusion imaging.

It is also predicted that future LSCI devices estimate more quantitative perfusion values thanks to deep learning and multi-exposure approaches [1]. In applications where the movement-induced error margins of handheld LSCI or those including excessive patient movements are not acceptable, the systems will become more robust in terms of movement artefacts benefiting from hardware-based artefact suppression (e.g. using a gimbal mount [2]) and artefact correction methods based on physical models, perhaps with image-analysis based estimation. Moreover, implementation of smartphone-based LSCI systems paves the way of point-of-care applications, such that the status and progress of a skin disorder can be monitored remotely via practitioners. Apart from its medical applications, it is expected that LSCI commercially enters the field of cosmetics and investigation of agricultural crops (e.g. apple and orange) where LSCI welcomes its new title: biospeckle method [3, 4]. 


\section{References}

[1] M. Hultman, M. Larsson, T. Strömberg, and I. Fredriksson, "Real-time video-rate perfusion imaging using multi-exposure laser speckle contrast imaging and machine learning", Journal of Biomedical Optics 25 (2020).

[2] B. Lertsakdadet, C. Dunn, A. Bahani, C. Crouzet, and B. Choi, "Handheld motion stabilized laser speckle imaging", Biomedical Optics Express 10, 5149 (2019).

[3] A. Zdunek, A. Adamiak, P. M. Pieczywek, and A. Kurenda, "The biospeckle method for the investigation of agricultural crops: A review", Optics and Lasers in Engineering 52, 276-285 (2014).

[4] R. Pandiselvam, V. P. Mayookha, A. Kothakota, S. V. Ramesh, R. Thirumdas, and P. Juvvi, "Biospeckle laser technique - A novel non-destructive approach for food quality and safety detection", Trends in Food Science and Technology 97, 1-13 (2020). 


\section{Summary}

In this thesis, the problem of movement artefacts in handheld laser speckle contrast perfusion imaging is addressed.

In Chapter 2, we quantified the movements of a handheld laser speckle contrast imaging (LSCI) system employing electromagnetic (EM) tracking and measured the applied translational, tilt and on-surface laser beam speeds. By observing speckle contrast on static objects, the magnitudes of translation and tilt of wavefronts were explored for various scattering levels of the objects. We concluded that for tissue mimicking static phantoms, on-surface speeds play a dominant role to wavefront tilt speed in creation of movement artefacts. The ratio of movement artefacts due to on-surface speed to those related to wavefront tilt speed depends on the optical properties of the phantom. Furthermore, with the same applied speed, the drop in the speckle contrast increases with decreasing reduced scattering coefficient, and hence the related movement artefact increases.

In Chapter 3, we studied the influence of the laser beam type in handheld-LSCI by evaluating the speckle contrast on static objects for beams with planar, spherical or scrambled wavefronts, and for movement artefacts caused by tilting or translation of wavefronts. We showed that the scrambled waves made by often-used engineered diffusers lead to significantly larger movement artefacts than planar or spherical waves. The planar waves caused the least movement artefacts when translating the LSCI system while the spherical waves caused the least movement artefacts when rotating the LSCI system.

In Chapter 4, we proposed an analytical-numerical model based on the optical Doppler effect for prediction of movement artefacts caused by translation in a handheld LSCI when static scattering objects are considered. The model incorporates the type of illumination as well as the imaging geometry by taking into account the spread of wavevectors for illumination and detection. We validated the model on numerically simulated speckle patterns. Results of the speckle simulation are in agreement with predictions of the numerical model for both uniform-circular and circular-Gaussian forms of the density functions of the incoming and outgoing wavevectors.

In Chapter 5, we examined the performance of handheld laser speckle contrast imaging (LSCI) measurements compared to mounted measurements, demonstrated in psoriatic skin. A pipeline was introduced to process, analyze and compare data of 
11 measurement pairs (mounted-handheld LSCI modes) operated on 5 patients and various skin locations. The on-surface speeds (i.e. speed of light beam movements on the surface) were quantified employing mean separation (MS) segmentation and enhanced correlation coefficient maximization (ECC). The average on-surface speeds were found to be 8.5 times greater in handheld mode compared to mounted mode. Alignment of perfusion frames before averaging sharpened temporally averaged perfusion maps, especially in the handheld case. The results showed that after proper post-processing, the handheld measurements are in agreement with the corresponding mounted measurements on a visual basis. The average movement-induced difference of estimated perfusion values on psoriasis lesions between handheld-mounted pairs was $16.4 \pm 9.3 \%$ (mean \pm std, $n=11$ ), median of $23.8 \%$ after the background correction.

In Chapter 6, we broadened our focus to the scientific field of tissue engineering and biofabrication. We used LSCI, side-stream dark field (SDF) microscopy and white light imaging to investigate the structural and blood flow information of developing vascular networks within an ex ovo chicken embryo chorioallantoic membrane (CAM) model. First, white-light imaging was used to map the complete vascular network. Second, the spatial and temporal fluctuations of blood flow in the corresponding vessels were non-invasively captured by LSCI. Third, the organization of capillaries, as well as fluid flow velocities, were determined based on SDF microscopy, which enabled the visualization of individual erythrocytes flowing through capillary networks. Based on this information, the fluid flow shear stresses within individual vessels were estimated by computational fluid dynamics simulations in order to get an understanding of the flow-associated mechanical signals within developing vasculature. In proof-ofprinciple experiments, we performed LSCI on biofabricated perfusable muscle tissue models and showed that LSCI is compatible with bioengineered tissues and can help to better understand vascular organization and flow perfusion. The application of LSCI and SDF on perfusable tissues enables tissue engineers to study the flow perfusion in a non-invasive fashion. Flow manipulation helps to tune the vascular organization with multiscale vasculature into specific organization and to design mechanically stable tissues. 


\section{Samenvatting}

In dit proefschrift wordt het probleem van bewegingsartefacten in handheld laser speckle contrast perfusie beeldvorming behandeld.

In hoofdstuk 2 hebben we de bewegingen gekwantificeerd van een handheld laser speckle contrast imaging (LSCI) systeem met behulp van een elektromagnetische (EM) volger. Daarna hebben we de translatie, en tilt van de laserbundel snelheden gemeten. We hebben ook de totale translatiesnelheid van de bundel op het weefselsoppervlak bepaald. Door het speckle contrast op statische media te observeren, zijn de amplitudes van translatie en tilt van golffronten onderzocht voor verschillende verstrooiingsniveaus van de media. We hebben geconcludeerd dat voor statische weefselfantomen, translatiesnelheden op het oppervlak een dominante rol spelen vergeleken met tiltsnelheid van het golffront bij het creëren van bewegingsartefacten. De verhouding van bewegingsartefacten door de snelheden op het oppervlak tot die ten gevolge van de tiltsnelheid hangt af van de optische eigenschappen van het fantoom. Bovendien neemt met dezelfde toegepaste snelheid, de daling van het speckle contrast toe met afnemende verstrooiingscoëfficiënt, en daarmee neemt het gerelateerde bewegingsartefact toe.

In hoofdstuk 3 hebben we de invloed van het type laserbundel in handheld-LSCI bestudeerd door het speckle contrast op statische media te evalueren voor bundels met vlakke, bolvormige of grillig gevormde golffronten, en voor bewegingsartefacten veroorzaakt door tilt of translatie van golffronten. We hebben laten zien dat de grillig gevormde golven gemaakt door vaak gebruikte optische diffusors leiden tot aanzienlijk grotere bewegingsartefacten dan vlakke of bolvormige golven. De vlakke golven veroorzaakten de minste bewegingsartefacten bij translatie van het LSCI-systeem, terwijl de bolvormige golven de minste bewegingsartefacten veroorzaakten bij het roteren van het LSCI-systeem.

In hoofdstuk 4 hebben we een analytisch-numeriek model gepresenteerd op basis van het optische Doppler-effect voor het voorspellen van bewegingsartefacten veroorzaakt door translatie in een draagbare LSCI, bij het meten op statisch verstrooiende media. Het model omvat zowel de geometrie van de belichting als de beeldvormende geometrie door rekening te houden met de spreiding van golfvectoren voor belichting en detectie. We hebben het model gevalideerd op numeriek gesimuleerde specklepatronen. De resultaten van de specklesimulatie zijn in overeen- 
stemming met de voorspellingen van het numerieke model voor zowel uniform-cirkel vormige als circulair-Gaussische vormen van de verdelingen van de inkomende en uitgaande golfvectoren.

In hoofdstuk 5 hebben we de haalbaarheid onderzocht van het uitvoeren van handheld metingen van LSCI gedemonstreerd in psoriatische huidlaesies. We hebben een pipeline geïntroduceerd om data van 11 meetparen te verwerken, te analyseren en te vergelijken in gemonteerde en draagbare modi van LSCI. Metingen zijn gedaan op 5 patiënten en verschillende huidlocaties. De snelheden op het oppervlak (d.w.z. snelheid van bewegingen van de lichtstraal op het oppervlak) werden gekwantificeerd met behulp van gemiddelde separation (MS) segmentatie en enhanced correlatiecoëfficiënt maximalisatie (ECC). De gemiddelde snelheid op het oppervlak bleek 8,5 keer hoger te zijn in de handheld-modus in vergelijking met de gemonteerde modus. Uitlijning van alle gemeten doorbloedingsverdelingen voorafgaand aan middeling verscherpte tijdsgemiddelde perfusieverdelingen vooral in de handheld modus. De resultaten toonden aan dat na een goede nabewerking de handmetingen op visuele basis overeenkomen met de bijbehorende gemonteerde metingen. De geschatte perfusiewaarden op psoriasislaesies na de achtergrond correctie in handheld-modus omvat een gemiddeld door beweging geïnduceerd verschil van $16,4 \pm 9,3 \%$ (gemiddelde \pm standaarddeviatie, $n=11$ ), mediaan van $23,8 \%$ in vergelijking met de gemonteerde metingen.

In hoofdstuk 6 hebben we onze focus verbreed nsaar het wetenschappelijke gebied van tissue engineering en biofabricage. We gebruikten LSCI, side-stream dark field (SDF) microscopie en witlichtbeeldvorming om de structurele en bloedstroominformatie van het ontwikkelen van vasculaire netwerken binnen een ex ovo kippenembryochorioallantoïsmembraanmodel te onderzoeken. Ten eerste hebben we beeldvorming met wit licht gebruikt om het volledige oppervlakkige vasculaire netwerk in kaart gebracht. Ten tweede hebben we de ruimtelijke en temporele fluctuaties van de bloedstromen in de corresponderende vaten niet-invasief gemeten door LSCI. Ten derde werd de organisatie van capillairen, evenals de vloeistofstroomsnelheden, bepaald op basis van SDF-microscopie, die de visualisatie van individuele erytrocyten mogelijk maakte die door capillaire netwerken stromen. Op basis van deze informatie zijn de schuifspanningen in de vloeistofstroom in individuele vaten geschat door computationele vloeistofdynamica-simulaties om inzicht te krijgen in de stroomgerelateerde mechanische signalen binnen het zich ontwikkelende vaatstelsel. In proof-of-principle experimenten, voerden we LSCI metingen uit op bio-gefabriceerde perfundeerbare spierweefselmodellen en toonden aan dat LSCI compatibel is met bio-ontworpen weefsels en kan helpen om de vasculaire organisatie en bloedperfusie beter te begrijpen. De toepassing van LSCI en SDF op perfundeerbare weefsels stelt tissue engineers in staat de perfusie op een niet-invasieve manier te bestuderen. Stromingsmanipulatie helpt om de vasculaire organisatie met grote en kleine vasculatuur af te stemmen op een specifieke organisatie en om mechanisch stabiele weefsels te ontwerpen. 


\section{Acknowledgements}

I would like to acknowledge every single one who has directly or indirectly helped me in the past four and a half year. Without the contribution of so many people around me, it would be impossible for me to reach this milestone.

Let me start by thanking my mother, Mitra, and my wife, Motahare. I am grateful to your boundless emotional support (this Latex package does not allow me to write some words in Farsi). I express my sincere appreciation to my supervisor Wiendelt who gave me the opportunity to join the group as a PhD student. You trusted me that I can do this work despite my rather different scientific background. And I have done my best to accomplish this. Ik waardeer uw constante ondersteuning in diverse aspecten: het leren van de taal, het onderzoeken, het manuscript schrijven etc. Ik ben trots op uw bijdrage aan hoofdstuk 4 en natuurlijk aan de rest van dit proefschrift. U hebt me geïnspireerd om sporten in mijn dagelijks leven toe te passen, zoals fietsen. Ook heb ik nu veel betere leven-werk balans.

Sylvia, vanaf de eerste moment dat ik was gesolliciteerd voor de $\mathrm{PhD}$ vacature tot nu heb je heel veel voor mij gedaan. Hartelijke dank voor alle hulp, advies en gezelligheid. Tom, je hebt me laten zien hoe een mooi en systematische meting uit te voeren. Je hebt me geleerd hoe een optische opstelling te bouwen. Wij hebben uren kritische discussies gehad over diverse aspecten van het project. Zonder jouw bijdrage zou hoofdstuk 3 niet worden gevormd. Hartelijk dank dat je er bent.

I have had awesome time at BMPI. I thank Altaf, Sjoukje and all of the BMPI members for the scientific discussions, feedback giving and social interactions. It was not easy for you to patiently listening to my often-given presentations at our group meetings. Thanks for teaching me things, passing me your experiences and letting me grow. Ivo, you challenged me in several scientific aspects. Gerwin, you taught me many optical concepts and programming skills when I just joined the group. You lent me your Alfred self-study piano book. Maura and David, you encouraged me speak Dutch and dared me to sing Farsi in a karaoke bar. I had nice time with Bart working in the same office and collaborating in the peppered skin project. Yoeri, Chapter 5 of this thesis would never form if you did not knock on the door in that day to ask for joining a coffee break. It was before the COVID-19 pandemic. Johan and Wilma, thanks for your guidance during the lab work.

I would like to take the time to acknowledge Miriam, Klaas, Tommie and Sven 
who joined our project to do their bachelor assignments. The same goes to Wilson who did his Capita Selecta project and master assignment with us.

Beril, Stijn, Mike, Sander and Gerben, I am proud that, together, we managed to initialize and develop our HAPI despite all the challenge and difficulties. Ferdi, your suggestion of using the EM tracker resulted in formation of Chapter 2 of this thesis. Mirjam and Marieke, working with you, I had my first interaction with medical research experts. Tjitske and Freek, thanks for being with us and examining our HAPI system. I also acknowledge the input and support of Onno and the members of our NWO user meetings. Prasanna, you are my first and the best Indian colleague. I did my first (and for sure the last because it is dangerous) tandem skydiving in Texel with you. You kept me motivated and encouraged me during our long and fruitful collaboration. I am proud of Chapter 6 of this thesis which is the result of all those experiments during weekends and late evenings. Jeroen, thanks for your kind support.

Amin, you were my first country mate I met in the Netherlands. Bahareh, Mahdi, Davoud, Ehsan, Sajjad, Vahid, Shahrooz, Hasan, Ali, Pouya, Salman, Reza, Mohammad, Hamed and Mojtaba (karbit) you are my memorial friends. Nhu Y, you were my first friendly colleague at BMPI. Lukasz, you encouraged me to join you for a $100 \mathrm{~km}$ cycling activity and introduced me the scuba diving. I am now recognized as an SSI advanced open water diver with 34 dives and 5 specialties. I thank my dive instructors Bas, Daan, Maurice, Stefan and Len at ZPV Piranha as well as (board) members of swimming, namely Mathijs, Jeroen, Thomas, Tamara, Rinke to provide the opportunity for attending the awsome NSZK matches. Francis, you made a plan for our three months preparation so I ran my first ever Marathon. I am now planning to do an iron man triathlon in the future. My special thank to the teachers of Dutch courses, namely Natasja, Jet, Gea, Carolina and Yvonne; and English courses, namely Dina and Marieke who helped me to improve my communication skills as well as social interactions.

All in all, I am happy that I am defending my thesis which is a great moment in my research career. I appreciate the time of the members of my graduation committee, namely, Prof. Sterenborg, Dr. Seyger, Prof. Offerhaus, Prof. Verdaasdonk and Dr. Tesselaar. 


\section{About the author}

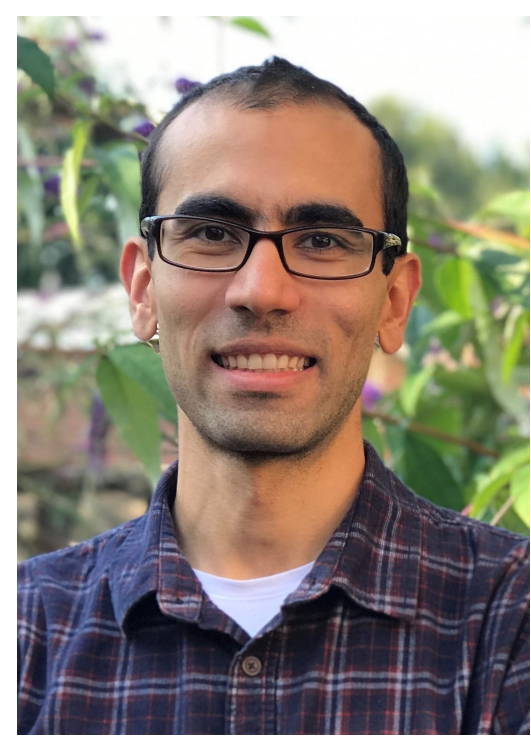

Ata Chizari was born in 1990 in Tehran, Iran. $\mathrm{He}$ received his BSc (hons) in Electronics in 2013 from Tafresh university, Tafresh, Iran, and his MSc (hons) in Communication Systems in 2015 from Shahid Beheshti University, Tehran, Iran. From 2014 till 2016 he served as a visitor research assistant at Optical Networks Research Lab, Sharif University of Technology, Tehran, Iran, where he contributed to Optical Wireless Communications and Photonics research topics. In 2017, he joined Biomedical Photonic Imaging Group, University of Twente, Enschede, The Netherlands as a PhD student where he investigates movement artefacts in handheld laser speckle contrast perfusion imaging. His research interests include laser speckle contrast imaging, laser Doppler perfusion imaging and speckle metrology. 

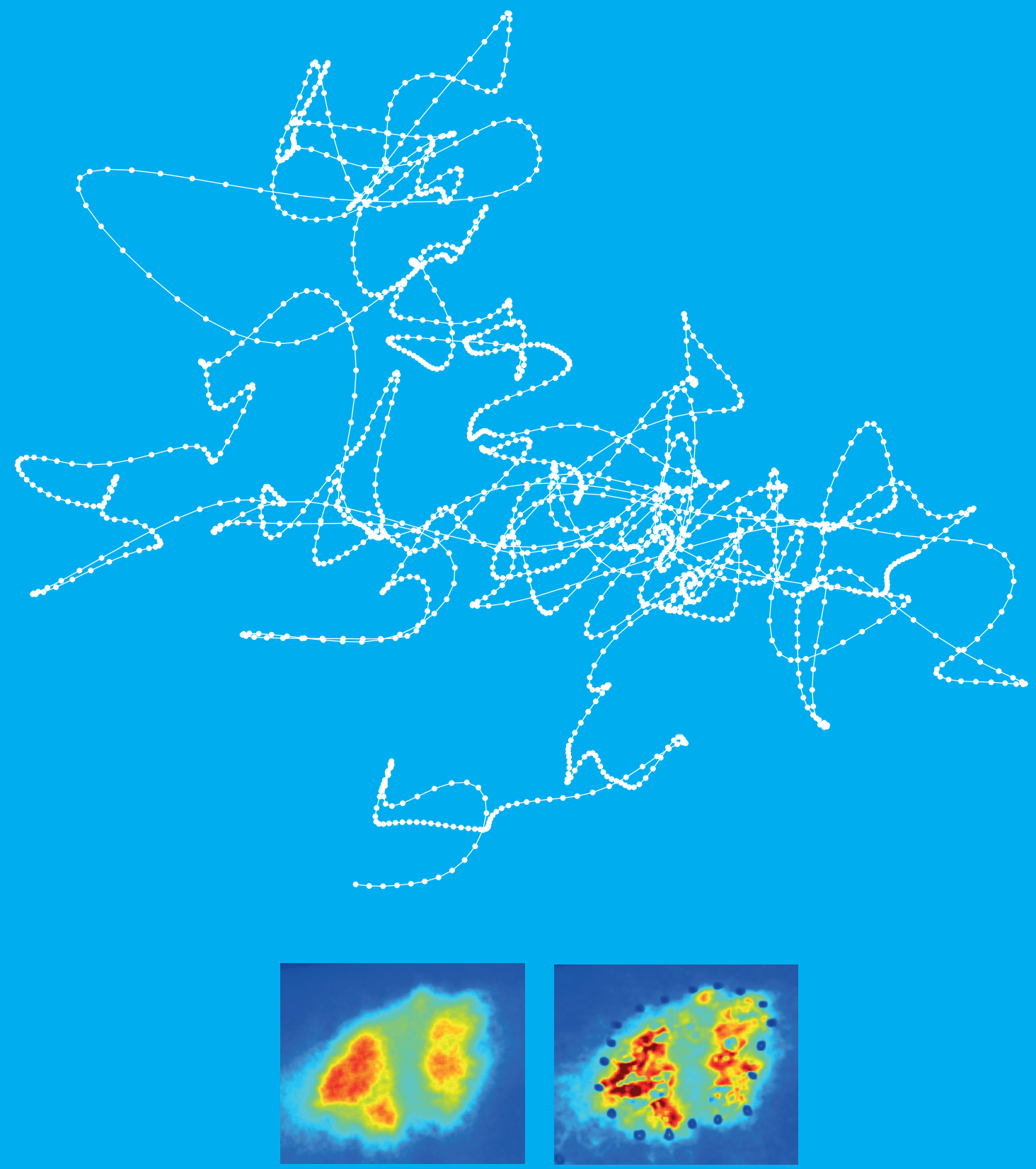